\title{
REVISÃo SISTEMÁTICA E PALEOBIOGEOGRÁFICA DE TRILOBITAS PHACOPIDA (HOMALONOTIDAE E CALMONIIDAE) DO DEVONIANO DAS BACIAS DO PARNAÍBA E AMAZONAS, BRASIL
}

\author{
Felipe van Enck Meira
}

\author{
Tese apresentada ao Programa de Pós- \\ Graduação em Geoquímica e Geotectônica \\ do Instituto de Geociências da Universidade \\ de São Paulo, como parte dos requisitos para \\ a obtenção do título de doutor.
}

Orientadora: Prof. Dra. Juliana de Moraes Leme

TESE DE DOUTORAMENTO

Programa de Pós-graduação em Geoquímica e Geotectônica

\section{São Paulo}


"Kites rise highest against the wind - not with it."

- Winston Churchill 


\section{Agradecimentos}

Agradeço a Deus, por sempre iluminar o caminho durante esses anos de altos e baixos do Doutorado. Agradeço a Ele também por enviar dois verdadeiros anjos da guarda à minha vida - minha esposa Angela Faleiros van Enck Meira e meu filho, Thomas Faleiros van Enck Meira.

Agradeço a meus pais, José Carlos e Sylvia, e à minha irmã, Patrícia, pelo apoio durante a jornada, ainda que à distância, por vezes. Sou grato aos meus sogros, Jair e Lucia, que sempre foram como verdadeiros pais e conselheiros.

À FAPESP (Processo $n^{\circ}$ 2012/07075-3), pelo suporte financeiro, sem o qual o Doutorado não seria viável.

À minha orientadora, Dr ${ }^{\mathrm{a}}$. Juliana de Moraes Leme, pela orientação, discussões e esclarecimentos pertinentes ao projeto.

Ao Doutorando Fabio Carbonaro e ao Dr. Renato Ghilardi (UNESP-Bauru), pela parceria e por discussões importantes na realização do trabalho.

À Dra . Niède Guidon (FUMDHAM), pelo empréstimo de fósseis da região de São João Vermelho (Piauí), estudados aqui.

Sou grato às seguintes pessoas, por permitirem meu acesso às instituições para visita de acervos, e por sua disponibilidade, atenção e ajuda durante minha permanência: Bushra Hussaini (AMNH), Flávia Alessandra Figueiredo, Mônica de Medina Coeli, Dr. Ismar de Souza Carvalho (IG-UFRJ), Dr. Mario Suárez-Riglos (MNKM, YPFB), Ana Paula Linhares Pereira, Dra ${ }^{\mathrm{a}}$. Maria Inês Ramos, Dr ${ }^{\mathrm{a}}$. Heloisa Maria Moraes Santos (MPEG), Rodrigo da Rocha Machado, Irma Tie Yamamoto e Dr ${ }^{\mathrm{a}}$. Rita de Cassia Tardin Cassab (MCT-DNPM), Dr. Antonio Carlos Sequeira Fernandes, Marcia Fernandes de Aquino Santos e Dra ${ }^{\text {a }}$ Vera Medina da Fonseca (MN-UFRJ).

À Dra . Maria da Glória Carvalho (AMNH), pelas discussões e esclarecimentos, em especial relacionados aos Homalonotidae. 
Aos funcionários do IGc-USP, Sandra e Erica (Biblioteca), Leonardo e Magali Poli (Secretaria de Pós-graduação), Zefinha (acervo de fósseis), Ivone (Oficina de Réplicas), Claudionor e Henrique (Gráfica). 


\section{SUMÁRIO}

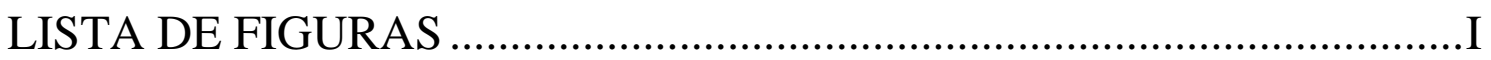

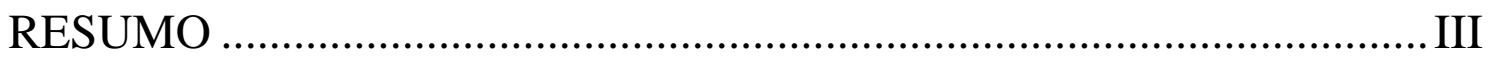

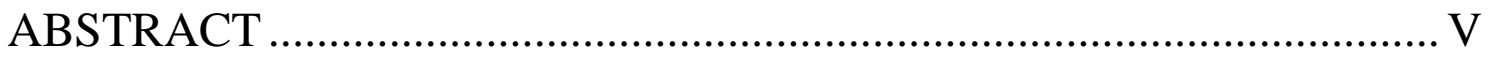

ESTRUTURA DA TESE …………………...................................... VII

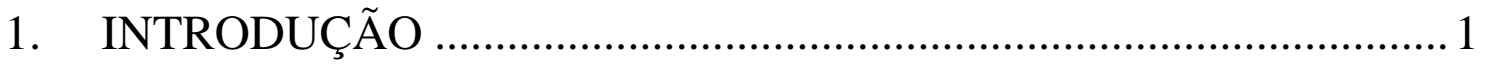

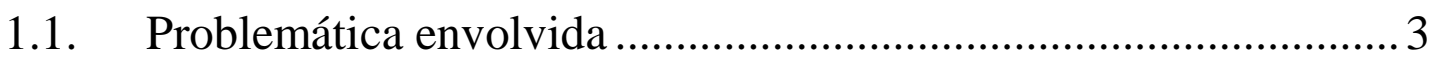

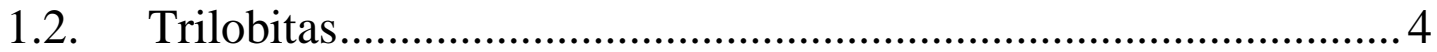

2. OBJETIVOS

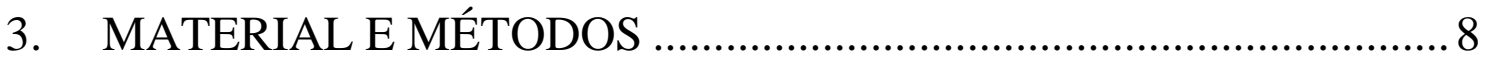

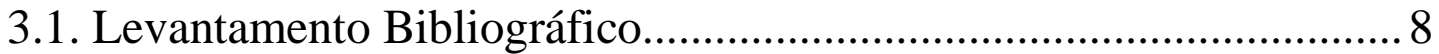

3.2. Coleções Examinadas (Anexo I) ..................................................... 8

3.3. Procedência das Amostras................................................................. 11

3.4. Preparação dos Fósseis.................................................................... 12

3.5. Descrição das Amostras ...................................................................... 12

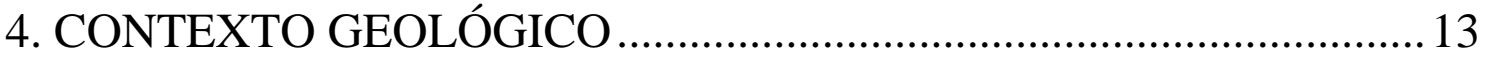

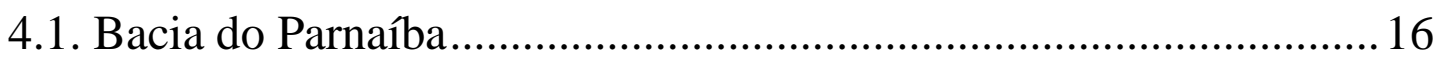

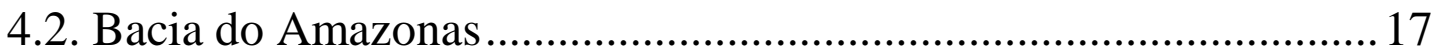

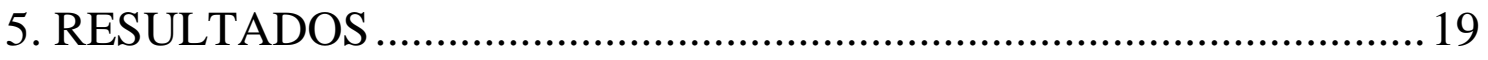

5.1. Revisão Sistemática (Anexos II, III, IV, V e VI)............................ 19

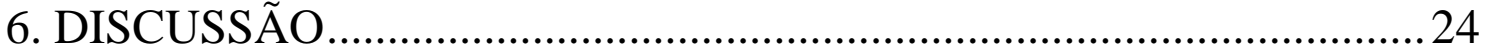

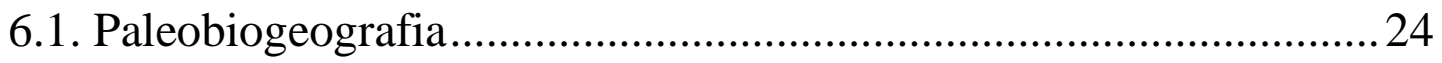

6.2. Rotas migratórias nas bacias do Parnaíba e Amazonas .................... 29

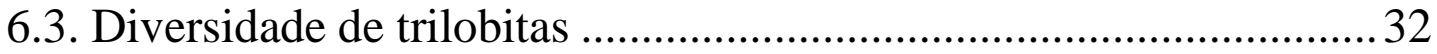

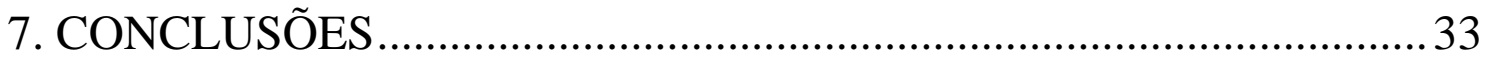

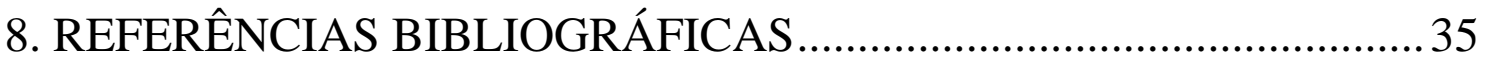

ANEXO I: Relação do material em estudo, depositado nas coleções nacionais e internacionais. 
ANEXO II: The “Metacryphaeus tuberculatus group” (trilobita, calmoniidae) from the devonian of the Parnaíba basin, Brazil

ANEXO III: Metacryphaeus tuberculatus (Kozlowski, 1923) and M. australis (Clarke, 1913) (Trilobita, Phacopida) from the Devonian of Paraná Basin: taxonomy and palaeobiogeography.

ANEXO IV: Systematic revision and paleobiogeography of calmoniid trilobites (Phacopida) from the Devonian of the Amazon Basin, Brazil

ANEXO V: The occurrence of Phacopida trilobites from Pimenteira Formation at João Costa, Piauí, Brazil. 


\section{LISTA DE FIGURAS}

Figura 1: Partes do corpo de um trilobita Homalonotidae (Trimerus). Modificado de Harrington et al. (1959).

Figura 2: Localização das faixas de afloramento de rochas sedimentares précarboníferas (em rosa) nas bacias do Amazonas e Parnaíba. Modificado de Schobbenhaus \& Brito Neves (2003). .14

Figura 3: Locais onde trilobitas Homalonotidae e Calmoniidae foram encontrados, nas bacias do Amazonas (área amarela indicada em B, segundo Silva \& Fonseca, 2005; Ponciano \& Machado, 2007; e Fonseca \& Ponciano, 2011) e do Parnaíba (afloramentos em municípios apresentados em C, segundo Carvalho, 1995; e Leme et al., 2011). Modificado de Faria et al. (2004) (B), e de Angelim et al. (2004) e Vasconcelos et al. (2004) (C)

Figura 4: Seção estratigráfica do Grupo Canindé. Extraído de Góes (1995).

Figura 5: Seção-tipo do Grupo Urupadi. Extraído de Cunha et al. (2007)

Figura 6: Mapa paleogeográfico do Devoniano, mostrando os domínios paleobiogeográficos. Modificado de www.palaeos.com .24

Figura 7: Reconstrução paleogeográfica do Brasil e áreas adjacentes no intervalo Neoemsiano-Eifeliano (final do Eodevoniano-início do Mesodevoniano), mostrando os cinturões de afloramento das três principais bacias paleozoicas brasileiras: Bacia do Amazonas (1), Bacia do Parnaíba (2) e Bacia do Paraná (3). Modificado de Melo (1988) 27

Figura 8: Reconstrução paleogeográfica do Brasil e áreas adjacentes no intervalo Neoeifeliano-Givetiano (Mesodevonaino). Indicações das bacias paleozoicas é a mesma da figura anterior. Modificado de Melo (1988) .28

Figura 9: Reconstrução da paleogeografia do Brasil e áreas adjacentes no intervalo Neoemsiano-Eifeliano (final do Eodevoniano-início do Mesodevoniano), mostrando as áreas de afloramentos das três principais bacias sedimentares paleozoicas brasileiras: 
Bacia do Amazonas (1), Bacia do Parnaíba (2) e Bacia do Paraná (3). Setas indicam o sentido das rotas migratórias de trilobitas Calmoniidae. Quadrados e círculos negros indicam, respectivamente, Calmoniidae do Emsiano da Bolívia e Calmoniidae do Eifeliano da Bacia do Amazonas. Modificado de Melo (1988)

Figura 10: Reconstrução da paleogeografia do Brasil e áreas adjacentes no intervalo Neoeifeliano-Eogivetiano (Mesodevoniano) do Brasil e áreas adjacentes. Setas indicam sentido das rotas migratórias de Calmoniidae do gênero Eldredgeia. Triângulos negros correspondem a ocorrências de Eldredgeia venusta no Emsino da Bolívia. Pentágonos negros representam o gênero Eldredgeia no Neoeifeliano-Eogivetiano das bacias do Parnaíba e Amazonas. Modificado de Melo (1988). 31 


\section{RESUMO}

O conhecimento acerca dos invertebrados devonianos no Brasil teve início em fins do século XIX e a primeira metade do século XX, período no qual foram feitas expedições pioneiras às principais bacias paleozoicas do país - as bacias do Paraná, Parnaíba e Amazonas. Desta fase também resultaram importantes trabalhos científicos (e.g., Clarke, 1913), usados ainda hoje como referência em estudos. Dentre os trilobitas, os grupos mais representativos são os Homalonotidae e os Calmoniidae, cuja importância como ferramentas em questões paleoambientais e paleobiogeográficas vem sendo constatada a partir de recentes revisões sistemáticas em fósseis da Bacia do Paraná. Estes estudos também têm demonstrado que muitas das variações nos fósseis, interpretadas por pesquisadores anteriores como taxonômicas, decorrem de alterações tafonômicas, que podem levar a classificações errôneas. Neste contexto, o presente trabalho teve como objetivos revisar a sistemática dos trilobitas Phacopida (Homalonotidae e Calmoniidae) nas bacias do Parnaíba e Amazonas, incluindo, na primeira localidade, a nova ocorrência de São João Vermelho, no município de João Costa (PI), investigar, sempre que pertinente, a implicação das alterações tafonômicas na sistemática desses fósseis, identificando fatores diagenéticos/intempéricos que podem interferir nos caracteres morfológicos e discutir as implicações paleobiogeográficas dos Homalonotidae e Calmoniidae do Devoniano das bacias do Amazonas e Parnaíba, em relação a outras localidades adjacentes à estas bacias. Resultados das pesquisas mostram que a localidade de São João Vermelho é bastante promissora do ponto de vista paleontológico, pois consiste em uma nova localidade para o táxon M. tuberculatus, o qual também é documentado nas duas subbacias da Bacia do Paraná - Alto Garças e Apucarana, indicando que esta espécie apresenta alto grau de cosmopolitanismo. A análise de exemplares de Burmeisteria (Homalonotidae) desta localidade sugere que as diferenças nas espécies deste gênero no Brasil são mais sutis do que imaginado. Na Bacia do Amazonas, os Phacopida estão representados especialmente por Calmoniidae do gênero Malvinella, também presentes na Bolívia. As relações paleobiogeográficas entre as bacias paleozoicas brasileiras e a Bolívia, durante o Eo e Mesodevoniano, teriam sido mais estreitas do que se imaginava. A principal rota de migração para a Bacia do Parnaíba teria sido através da Bacia do Paraná, conforme constatado pela presença de M. tuberculatus. Já a migração para a Bacia do Amazonas 
teria se dado a sudoeste desta bacia, já que Malvinella ocorre apenas nesta bacia e na Bolívia.

Palavras-Chave: Homalonotidae; Calmoniidae; Devoniano; Bacia do Parnaíba; Bacia do Amazonas; Paleobiogeografia. 
The knowledge on Brazilian Devonian invertebrates started by the end of the $19^{\text {th }}$ century and the first half of the $20^{\text {th }}$ century, period which pioneering expeditions were made to the main Paleozoic basins of the country - Paraná, Parnaíba and Amazon basins. Important scientific contributions also resulted from this fase (e.g. Clarke, 1913), still used today as reference in studies. Among trilobites, the most representative groups are the Homalonotidae and Calmoniidae, whose importance in paleoenvironmental and paleobiogeographical questions have been noted from recent systematic revisions in Paraná Basin fossils. These studies also have demonstrated that many variations in fossils, interpreted by previous researchers as taxonomic, are due to taphonomic alterations, which can lead to erroneous classifications. In this context, the current study aimed to review the systematics of Phacopida trilobites (Homalonotidae and Calmoniidae) from the Parnaíba and Amazon basins, including, in the former locality, the new occurrence of São João Vermelho, in the João Costa municipality (PI); to investigate, whenever relevant, the implication of the taphonomic alterations in the systematics of these fossils, identifying diagenetic/weathering factors which can interfere in the morphological features; and to discuss the paleobiogeographic implications of the Homalonotidae and Calmoniidae from the Devonian of the Parnaíba and Amazon basins, in relation to other localities adjacent to these basins. Study results show that the locality of São João Vermelho is paleontologically promising, as it consists of a new place for the M. tuberculatus taxon, which is also documented in the both Paraná Basin subbasins - Alto Garças and Apucarana, indicating that this species is highly cosmopolitan. The analysis of Burmeisteria (Homalonotidae) exemplars from this locality suggests that differences between the species of this genus in Brazil are slighter than thought. In the Amazon Basin, Phacopida trilobites are especially represented by the Calmoniidae genus Malvinella, also present in Bolivia. The paleobiogeographic relations between the Paleozoic Brazilian basins and Bolivia, during Early and Middle Devonian, would have been closer than thought. The main migration route to the Parnaíba Basin would have been through the Paraná Basin, as found by the presence of $M$. tuberculatus. The migration to the Amazon Basin, in turn, 
would have occurred southwestward this basin, as Malvinella occurs only in this locality and in Bolivia.

Keywords: Homalonotidae; Calmoniidae; Devonian; Parnaíba Basin; Amazon Basin; Paleobiogeography. 


\section{ESTRUTURA DA TESE}

A presente tese está apresentada em forma de artigos científicos, respeitando as regras do Regimento da Pós-Graduação da USP e das Normas do Programa de PósGraduação em Geoquímica e Geotectônica, IGc/USP. Isso é, todo o documento deverá ser redigido em um único idioma (inglês ou português). Em caso de inclusão de artigos aceitos e/ou submetidos em inglês, no corpo de texto, toda a dissertação/tese deverá ser em inglês. Os artigos aceitos e/ou submetidos também poderão ser incluídos na forma de anexo, podendo ser o corpo do texto redigido em português. Dessa forma, os artigos são apresentados em anexos, ao final do corpo da tese, o qual é composto pelos itens: Introdução, Objetivos, Materiais e Métodos, Resultados, Discussão e Conclusões.

Os resultados correspondem aos artigos científicos em inglês apresentados como anexos. Dois deles já foram publicados. Um no periódico Geologia USP e outro está aceito para publicação no periódico Ameghiniana, um artigo está submetido, um artigo finalizados e ainda não submetido, e outro ainda em preparação. 


\section{INTRODUÇÃO}

O conhecimento acerca dos trilobitas na América do Sul se deve às expedições feitas por naturalistas europeus e norte-americanos a este continente, no século XIX. No Brasil, descrições pioneiras sobre estes fósseis foram realizadas na segunda metade do século XIX, com os trabalhos de Hartt \& Rathbun (1875), Clarke (1890) e Katzer (1898) sobre a fauna devoniana da Bacia do Amazonas. A partir do início do século XX, com a publicação da clássica monografia de John M. Clarke (1913), sobre os fósseis devonianos da Bacia do Paraná, e sua correlação com faunas contemporâneas da África do Sul e Ilhas Falkland, o enfoque dos estudos sobre os trilobitas brasileiros passou a ser a Bacia do Paraná, em especial sua porção oriental (Sub-bacia Apucarana) (Lange, 1954; Lange \& Petri, 1967). A partir dos anos 80, após um período de escassez nos estudos de trilobitas brasileiros, este tema voltou a ser enfocado, através das pesquisadoras Maria da Glória Pires de Carvalho e Maria Terezinha Barcellos-Popp (Ghilardi \& Simões, 2007). Enquanto a primeira ocupou-se mais em estudar os trilobitas das bacias do Amazonas e Parnaíba (Carvalho, 1995), Popp voltou-se aos fósseis da Sub-bacia Apucarana, em especial à família Calmoniidae (Popp, 1985), em função da importância despendida a este grupo como elemento endêmico do Domínio Malvinocáfrico, conforme expressado em Eldredge \& Ormiston (1979) e Eldredge \& Braniša (1980).

Na última década, as contribuições de Soares (2007), Soares et al. (2008a, b) e Simões et al. (2009) destacaram-se por consistirem em revisões sistemáticas de trilobitas da Bacia do Paraná, utilizando como método o estudo das alterações causadas por agentes diagenéticos e intempéricos, e como estas modificações afetam a morfologia dos fósseis e, consequentemente, as interpretações taxonômicas e sistemáticas. Além disso, parte destes estudos (Soares, 2007; Simões et al., 2009) enfocaram a revisão sistemática da espécie Burmeisteria notica (família Homalonotidae) que, diferentemente dos Calmoniidae, não havia sido atualizada desde sua descrição, por Clarke (1913). Os resultados destas pesquisas mostraram que a diagnose de B. notica, feita por Clarke (1913), baseava-se em caracteres presentes nesta espécie, e em outro táxon, Burmeisteria herscheli, até então desconhecido no Paleozoico brasileiro, mas presente na Bolívia, África do Sul e Ilhas Falkland. Com 
isso, Simões et al. (2009) estabeleceram uma descrição atualizada para $B$. notica e $B$. herscheli, e constataram a presença da segunda espécie na Bacia do Paraná. Do mesmo modo, elementos atribuídos a Metacryphaeus rotundatus, encontrado em depósitos coevais na Bolívia, foram constatados por Soares et al. (2008a), na Bacia do Paraná.

Contrariamente à frequiência de publicações voltadas aos trilobitas da Bacia do Paraná, seu estudo nas demais bacias paleozoicas brasileiras é esparso. Na Bacia do Parnaíba, Kegel (1953) atribuiu aos gêneros Homalonotus e Asteropyge trilobitas encontrados na Formação Pimenteira. O gênero Asteropyge também foi conferido a exemplares descobertos no Membro Passagem e Formação Longá. Posteriormente, Castro (1968) revisou os fósseis da Formação Pimenteira e, com base em comparações com trilobitas da Bacia do Paraná, concluiu que o material assinalado por Kegel correspondia, respectivamente, às espécies Burmeisteria notica e Metacryphaeus australis. Nas décadas de 80 e 90, a pesquisadora Maria da Glória Carvalho revisou o material da Bacia do Parnaíba, e concluiu que os fósseis atribuídos a Metacryphaeus diferiam da espécie da Bacia do Paraná (Carvalho, 1995). Carvalho et al. (1997) publicaram a descrição das novas espécies, nomeando o material encontrado na Formação Pimenteira como Metacryphaeus kegeli, e Metacryphaeus meloi para os fósseis presentes no Membro Passagem (topo da Formação Pimenteira). Na Formação Longá, as amostras também foram atribuídas ao gênero Metacryphaeus, mas sua classificação específica não foi possível, devido à pequena quantidade de exemplares e preservação (Carvalho, 1995).

Na Bacia do Amazonas, parte do material cuja posição sistemática foi proposta em trabalhos pioneiros (Hartt \& Rathbun, 1875; Clarke, 1890; Katzer, 1898) foi revisado com base em amostras do Devoniano da Bolívia, por Eldredge \& Ormiston (1979). Estes autores sugeriram que alguns táxons inicialmente assinalados aos gêneros Dalmanites, Phacops e Homalonotus, pertenceriam, ou estariam relacionados, a gêneros bolivianos da família Calmoniidae, encontrados na Bolívia (Vogesina, Pennaia, Malvinella, Tarijactinoides e Metacryphaeus). Esta proposta sistemática é apresentada como uma lista em Eldredge \& Ormiston (1979). Revisões sistemáticas e descrições mais recentes, envolvendo os Calmoniidae da Bolívia, foram realizadas por Lieberman et al. (1991), Lieberman (1993) e Carvalho et al. (2003). Nestes trabalhos, os autores descrevem novos gêneros (Palpebrops, Eldredgeia, Gemelloides), e posicionam alguns elementos da Bacia do Amazonas neles ("Palpebrops" goeldi, Eldredgeia paituna, 
“Gemelloides" gemellus). Em 2007, Carvalho \& Fonseca revisaram a espécie Dalmanites maecurua (Clarke, 1890), e concluíram tratar-se de um elemento à parte da família Dalmanitidae. Estas autoras elegeram um novo gênero para este fóssil Amazonaspis - e associaram o material à família Synphoridae.

\subsection{Problemática envolvida}

De acordo com as informações disponíveis na literatura (Castro, 1968; Lieberman, 1993; Carvalho, 1995, 1999; Carvalho et al., 1997), as espécies de trilobitas presentes no Devoniano da Bacia do Parnaíba são:

- Burmeisteria notica, Metacryphaeus kegeli e Eldredgeia cf. venusta, na Formação Pimenteira;

- Metacryphaeus meloi, no Membro Passagem (base da Formação Pimenteira);

- Metacryphaeus sp., na Formação Longá.

Além destes táxons, Lieberman et al. (1991) relataram a ocorrência de Metacryphaeus tuberculatus, espécie presente no Devoniano da Bolívia, com base em um espécime da Formação Pimenteira. Carvalho (1995, 1999) e Carvalho et al. (1997), por outro lado, contestaram a proveniência desta amostra, alegando que a concreção na qual o espécime se encontra diferia da matriz em que os fósseis em geral ocorrem.

Na Bacia do Amazonas, os trilobitas devonianos compõem-se de representantes recentemente revisados (e.g., Amazonaspis maecurua, Carvalho \& Fonseca, 2007; Digonus derbyi, Carvalho \& Ponciano, 2015), com a nomenclatura em aberto (e.g., Phacops? pullinus, Clarke, 1890; Tarijactinoides? acanthurus, Eldredge \& Ormiston, 1979; "Pennaia" menurus, Cooper, 1982), e não revisados, desde sua descrição original (e.g., Phacops macropyge, Clarke, 1890).

Com base nas revisões de trilobitas do Domínio Malvinocáfrico (Eldredge \& Braniša, 1980; Cooper, 1982; Lieberman et al., 1991; Lieberman, 1993) e, mais recentemente, trilobitas Homalonotidae de bacias brasileiras (Soares, 2007; Simões et al., 2009; Carvalho \& Ponciano, 2015), nota-se que: 
a) A revisão das descrições de Clarke (1913) implica não apenas na revisão dos trilobitas por ele estudados, mas também naqueles de outras localidades e cujos autores basearam-se em sua monografia para descrevê-los;

b) Uma revisão dos táxons da Bacia do Amazonas, notadamente aqueles não reestudados desde sua descrição original era necessária, ainda mais se considerarmos as recentes revisões sob a perspectiva das modificações tafonômicas (e.g., Simões et al., 2009; Mori, 2013).

O presente trabalho revisou a sistemática dos Homalonotidae e Calmoniidae das bacias do Parnaíba e Amazonas que, por sua vez, permitiu correlações paleobiogeográficas mais acuradas com a fauna devoniana de outras localidades, como Bolívia, África do Sul e Ilhas Falkland.

\subsection{Trilobitas}

O Paleozoico foi marcado pelo surgimento e evolução de diversos grupos de invertebrados, dentre os quais os trilobitas foram, sem dúvida, dos mais representativos. Estes artrópodes, filogeneticamente posicionados como uma linhagem basal de Mandibulata (Scholtz \& Edgecombe, 2005) têm seu nome devido à presença, na superfície dorsal do corpo, de dois sulcos longitudinais, denominados sulcos axiais, que definiam três lobos: um lobo central, mais elevado, chamado lobo axial, e dois lobos adjacentes, os lobos pleurais (Fig. 1). O exoesqueleto, de natureza quitinosa, era formado por unidades mineralizadas, denominados escleritos, que compunham uma única peça fusionada na região anterior, o céfalo. Na região posterior, os escleritos formavam outra peça, o pigídio. Entre o céfalo e o pigídio, havia o tórax, composto por escleritos individualizados, articulados (Fig. 1) (Levi-Setti, 1995; Whittington et al., 1997; Clarkson, 1998; Carvalho et al., 2004).

O céfalo consiste na parte do corpo dos trilobitas com maior importância para os estudos ligados à morfologia. Em sua margem anterior, o céfalo pode ter um contorno semicircular a ogival, sendo reto posteriormente. O ângulo formado entre as margens laterais e a margem posterior é chamado ângulo genal. Os ângulos genais podem ser arredondados, ou terminar em uma projeção aguda, o espinho genal (Levi-Setti, 1995; Whittington et al., 1997). 


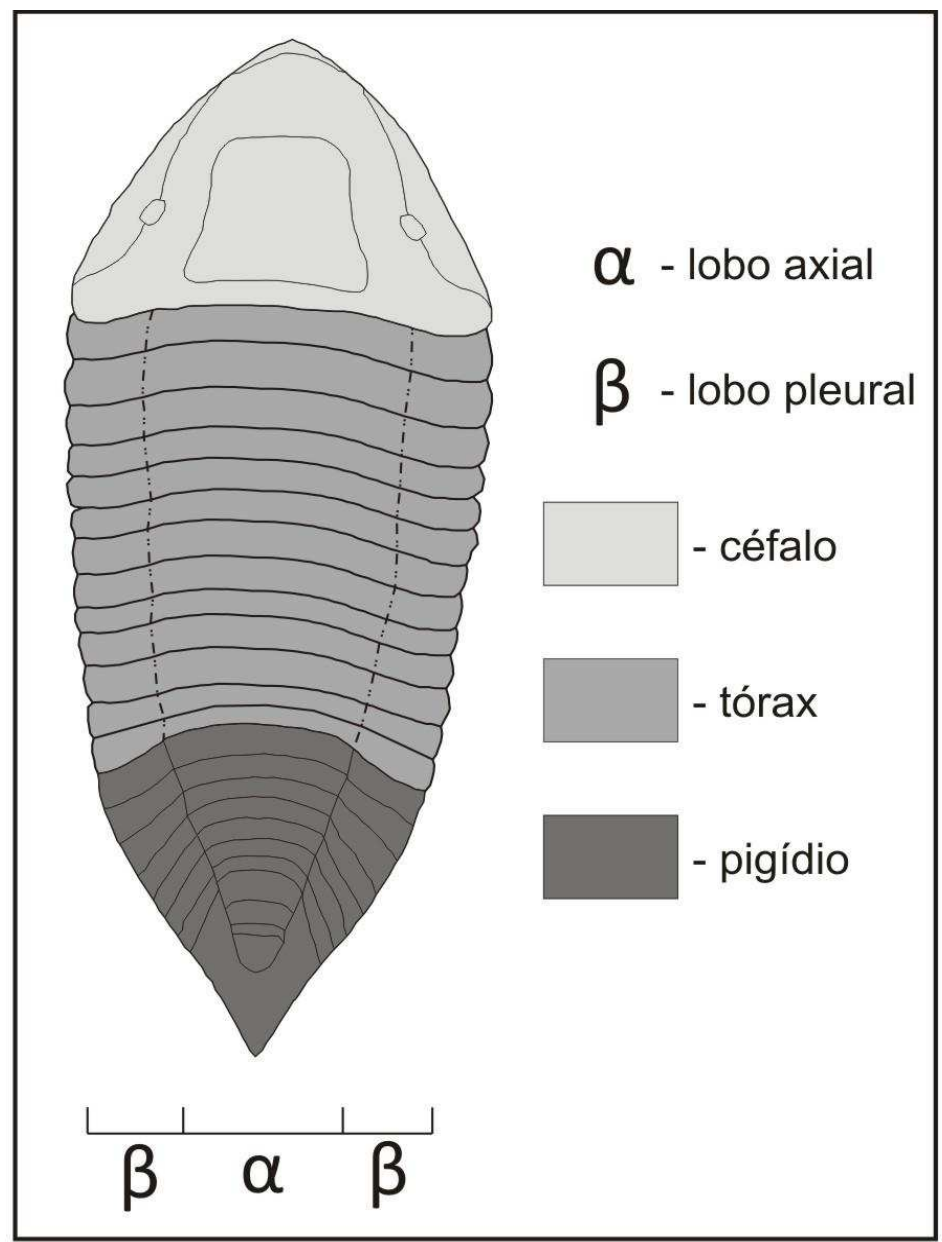

Figura 1: Partes do corpo de um trilobita Homalonotidae (Trimerus). Modificado de Harrington et al. (1959).

A porção cefálica do lobo axial recebe o nome de glabela, uma estrutura inflada que pode se estender anteriormente, além da margem anterior, ou terminar antes desta margem. A glabela pode apresentar pares de sulcos em sua superfície, os sulcos glabelares laterais. Posteriormente a estes sulcos, há um sulco transversal, denominado sulco occipital. Segundo a nomenclatura estabelecida por Whittington et al. (1997), os sulcos são designados pela letra "S" e por um número, a partir do sulco occipital. Estes sulcos delineiam lobos glabelares, os quais são nomeados pela letra "L" seguida de um número, tendo como origem o anel occipital. O último lobo, denominado lobo anterior, recebe a sigla LA (Whittington et al., 1997). Adjacente à glabela estão as genas, que são divididas pelas suturas faciais em fixigenas (ou genas fixas) e 
librigenas (ou genas livres). As primeiras formam, em conjunto com a glabela, uma estrutura denominada cranídio (Levi-Setti, 1995; Whittington et al., 1997). Um critério para classificar os trilobitas é através do curso dos ramos posteriores das suturas faciais, porção da sutura facial localizada após o olho do trilobita. Em suturas do tipo opistopária, os ramos posteriores das suturas faciais cortam a margem posterior do céfalo; quando estes ramos cortam as margens laterais, a sutura é denominada propária. Um terceiro tipo, gonatopárias, ocorre quando as suturas faciais cortam os ângulos genais. Há também trilobitas sem suturas faciais (Levi-Setti, 1995; Carvalho et al., 2004).

O tórax dos trilobitas é composto por um número variável (entre 2 e 42, segundo Levi-Setti, 1995) de peças individualizadas, os tergitos, que possuem articulação entre si, permitindo ao animal proteger sua parte ventral, não mineralizada, através do enrolamento do corpo. Cada tergito apresenta, na região mediana, uma porção mais elevada, o anel axial, limitado pelos sulcos axiais. Adjacente a este anel, estão as pleuras, as quais podem apresentar terminações arredondadas, ou projeções agudas, encurvadas para baixo e para trás, denominadas espinhos pleurais. Em geral, as pleuras apresentam sulcos pleurais, que podem ter servido para tornar estas estruturas mais fortes. A articulação entre um tergito e outro ocorre através de uma extensão localizada anteriormente nos anéis axiais, os meio-anéis articuláveis (articulating half-rings). Esta extensão se insere embaixo do anel anterior, em sua margem posterior (Levi-Setti, 1995).

A proporção entre o céfalo e o pigídio também pode ser usada como classificação para os trilobitas. A condição na qual o pigídio é proporcionalmente equivalente ao céfalo é chamada isopígio. Quando o pigídio é maior do que o céfalo, o termo aplicado é macropígio. Finalmente, trilobitas micropígios são aqueles que possuem o pigídio menor do que o céfalo (Carvalho et al., 2004). Na maioria dos trilobitas, o lobo axial estende-se até o pigídio, podendo atingir sua margem posterior, ou mesmo desdobrar-se além desta, formando um espinho pigidial terminal. Assim como no tórax, o lobo axial é composto por anéis axiais, embora no caso do pigídio eles estejam fusionados. As pleuras podem apresentar sulcos e costelas pleurais, que podem chegar às margens laterais. Espinhos marginais estão frequentemente presentes; em alguns casos, estas projeções não têm relação com o número de costelas das pleuras (Levi-Setti, 1995; Whittington et al., 1997). 
Ventralmente, as partes mineralizadas estavam restritas a inflexões das margens do céfalo e pigídio, e das porções distais das pleras do tórax; estas extensões são conhecidas como dobras (doublure). No céfalo, outra estrutura mineralizada é o hipóstoma, uma peça que pode ou não estar fusionada à dobra da margem anterior. Os trilobitas dispunham de apêndices birramosos ao longo de sua porção ventral, desde o céfalo até o pigídio. Um ramo era destinado à locomoção, e o outro, à respiração; no céfalo também havia um par de antenas, unirramosas (Fig. 1) (Carvalho, 1995; Clarkson, 1998; Carvalho et al., 2004).

O desenvolvimento ontogenético dos trilobitas é composto por três fases distintas. A inicial, as larvas destes artrópodes são denominadas protaspis. Apresentam entre 0,3 e 1mm de tamanho, e são compostas apenas pelo céfalo e pigídio. Na fase seguinte, meraspis, caracteriza-se pelo desenvolvimento dos segmentos torácicos. Na fase adulta, o trilobita é denominado holaspis, e seu tamanho aumenta conforme ocorrem as sucessivas mudas. Logo, o tamanho do animal está relacionado à sua idade (Levi-Setti, 1995). Existem ao menos dois modos pelos quais os trilobitas realizam a muda. O primeiro é o modo phacopida (Fig. XX), no qual o céfalo se desconecta do tórax entre o anel occipital e o primeiro segmento torácico. Ao sair da antiga muda, o animal força o céfalo desta muda a virar-se de ponta-cabeça. Outra forma de realizar a muda é pelo modo ollenida, no qual as librigenas se desconectam do cranídio, pelas suturas faciais (Levi-Setti, 1995; Whittington et al., 1997; Carvalho et al., 2004). 


\section{OBJETIVOS}

O presente estudo teve como objetivos: a) revisar a sistemática dos trilobitas Phacopida (Homalonotidae e Calmoniidae) nas bacias do Parnaíba, incluindo as novas ocorrências no município de João Costa (PI), e do Amazonas, que ainda careciam de alguma revisão mais recente; b) investigar, sempre que possível, a implicação das alterações tafonômicas na sistemática desses fósseis, identificando fatores diagenéticos/intempéricos que podem interferir nos caracteres morfológicos; c) discutir as implicações paleobiogeográficas dos Homalonotidae e Calmoniidae do Devoniano das bacias do Amazonas e Parnaíba, em relação a outras localidades adjacentes à estas bacias.

\section{MATERIAL E MÉTODOS}

\subsection{Levantamento Bibliográfico}

O levantamento bibliográfico foi executado inicialmente, a partir das publicações mais abrangentes e todas as referências foram catalogadas. Parte do material já estava à disposição, como acervo pessoal da Orientadora.

Em seguida, foram utilizados programas de busca da Internet, para busca de períodos online de acesso livre (American Museum Novitates, Revista Brasileira de Paleontologia, Geologia USP - Série Científica, etc.), e de acesso restrito (Journal of Paleontology, Palaeontology, etc.). Referências não localizadas na Internet foram obtidas no acervo da biblioteca do IGc-USP.

\subsection{Coleções Examinadas (Anexo I)}

Foram visitadas instituições em Santa Cruz de La Sierra - Bolívia (Museo de Historia Natural Noel Kempff Mercado - MNKM e Yacimientos Petrolíferos Fiscales Bolivianos - YPFB), para análise de exemplares provenientes da Bolívia e no Rio de Janeiro (MN/UFRJ, IGEO/UFRJ e MCT/DNPM), para análise de exemplares provenientes das bacias do Parnaíba e Amazonas. Adicionalmente, foram visitadas 
instituições em Nova York (AMNH), para análise de exemplares provenientes da Bacia do Parnaíba. Espécimes oriundos do Grupo Hamilton, Devoniano do Estado de New York, do Grupo Niagara, NY, das ilhas Falklands e Bolívia também foram analisados, para comparação. Em São Paulo, as dependências do Instituto de Geociências da USP foram usadas para análise de amostras cedidas pela Fundação Museu do Homem Americano (FUMDHAM). No Anexo I, a relação dos espécimes em estudo está discriminada.

\section{Museu de Ciências da Terra do Departamento Nacional de Produção Mineral}

\section{$\underline{\text { (MCT/DNPM)/Rio de Janeiro }}$}

Foram analisados 10 fósseis, coletados em sua maioria pelo geólogo Wilhelm Kegel (DNPM) na área das cidades de Picos e Pimenteiras (PI), provenientes das formações Pimenteira e Membro Passagem. Também se estudou exemplares da Bacia do Paraná $(\mathrm{n}=20)$.

$\underline{\text { Museu Nacional da Universidade Federal do Rio de Janeiro (MN/UFRJ)/Rio de Janeiro }}$

Nesta instituição, foram analisados 23 exemplares das formações Maecuru e Ererê (Bacia do Amazonas), dos quais 11 são holótipos, coletados pela Comissão Geológica do Império, na segunda metade do século XIX. Adicionalmente, foram analisados 10 exemplares de Homalonotidae (Trimerus delphinocephalus e Dipleura dekayi), provenientes do Grupo Hamilton, Devoniano do Estado de New York.

Instituto de Geociências da Universidade Federal do Rio de Janeiro (IGEO/UFRJ)/Rio de Janeiro

Foram estudados 16 espécimes de trilobitas Calmoniidae do Membro Passagem, coletados pela Dra. Maria da Glória de Carvalho (AMNH/New York) e pelo geólogo José Henrique Gonçalves de Melo (CENPES/PETROBRAS), dos quais quatro são parátipos da espécie Metacryphaeus meloi, além de 15 exemplares da Formação 
Maecuru, coletados por José Henrique Gonçalves de Melo em 1986. Alguns exemplares provenientes da região de Jaguariaíva (PR) foram adicionados à análise ( $\mathrm{n}=22)$.

\section{American Museum of Natural History (AMNH)/NEW YORK}

O material consiste em 74 espécimes de trilobitas Homalonotidae e Calmoniidae (formações Icla, Belén e Sica Sica) da Bolívia e 2 exemplares de Phacopidae (Viaphacops), coletados na região de Pujravi, Bolívia. Nesta instituição também foram analisados 2 exemplares de Metacryphaeus, provenientes da Formação Pimenteira (incluindo Membro Passagem), coletados na região de Picos (PI). Para comparação, foram analisados 18 exemplares de trilobitas Homalonotidae provenientes do Grupo Hamilton, Devoniano do Estado de New York e do Grupo Niagara, NY. Adicionalmente, foram analisados 7 exemplares de Homalonotidae (Burmeisteria herscheli) provenientes das Ilhas Falklands.

\section{Museu Emílio Goeldi/Belém}

O material consiste em 36 exemplares de Metacryphaeus meloi do Membro Passagem, coletados na região da cidade de Picos (PI). Nesta instituição, também foi analisado 1 exemplar de Phacops scirpeus da formação Maecuru (Bacia do Amazonas).

\section{$\underline{\text { Museo de Historia Natural Noel Kempff Mercado - MNKM }}$}

O material consiste em 32 espécimes de trilobitas Homalonotidae (Dipleura dekayi), coletados na região de Patacamaya - Chacoma, Bolívia. Nesta instituição também foram analisados 33 espécimes de trilobitas Calmoniidae, sendo 20 exemplares de Malvinella, 9 exemplares de Pennaia, 3 exemplares de Malvinocooperella, 5 exemplares de Metacryphaeus giganteus, 21 exemplares de Metacryphaeus tuberculatus e 5 exemplares de Eldredgeia venusta. Adicionalmente, foi analisado 1 exemplar de Metacryphaeus curvigena e 1 exemplar de Vogesina.

$\underline{\text { Yacimientos Petrolíferos Fiscales Bolivianos - YPFB }}$ 
Foram estudados 33 espécimes de trilobitas Homalonotidae da Bolívia, dos quais 16 são da espécie Dipleura dekayi. Nesta Instituição também foram analisados 14 espécimes de trilobitas Calmoniidae, sendo 2 exemplares de Metacryphaeus caffer, 3 exemplares de Pennaia, 3 de Plesiomalvinella e 3 exemplares de Eldredgeia venusta. Adicionalmente, foi analisado 1 exemplar de Metacryphaeus curvigena, 1 exemplar de Vogesina e 1 exemplar de Tarijactinoides.

\subsection{Procedência das Amostras}

O material em estudo proveniente da Bacia do Parnaíba, e depositado nas coleções do Museu de Ciências da Terra (MCT/DNPM), Instituto de Geociências da UFRJ (IGEO/UFRJ), American Museum of Natural History (AMNH) e Museu Paraense Emílio Goeldi (MPEG), foi coletado na região das cidades de Picos e Pimenteiras, a leste do Estado do Piauí, e são da Formação Pimenteira, de idade mesodevoniana (Neoeifeliano - Eogivetiano, Melo, 1985; Grahn \& Melo, 2004), e do Membro Passagem, mesodevoniana (Givetiano, Grahn, 1992). Já as amostras pertencentes à Fundação Museu do Homem Americano (FUMDHAM) foram encontradas na região da cidade de João Costa, a sudeste deste estado, e pertencem à Formação Pimenteira.

No Museu Paraense Emílio Goeldi também foram observados fósseis da Formação Maecuru, da Bacia do Amazonas (Neoemsiano - Neoeifeliano, Melo \& Loboziak, 2003; Grahn \& Melo, 2004).

Amostras das formações Maecuru e Ererê (Bacia do Amazonas) estão depositadas no Museu Nacional da UFRJ (MN/UFRJ), onde estão também os exemplares-tipo descritos por Clarke (1890), e coletados pela Comissão Geológica do Império (Silva \& Fonseca, 2005). No IGEO/UFRJ, se encontram apenas fósseis da Formação Maecuru, coletados em diferentes pontos do Rio Maecuru pela Expedição Orville A. Derby, realizada pela PETROBRAS, em 1986 (Silva \& Fonseca, 2005). Embora as amostras da Formação Ererê, coletadas no século XIX, não apresentem uma localização geográfica precisa, supõem-se que elas venham da área-tipo da Formação Ererê, no município de Monte Alegre (Oliveira \& Leonardos, 1978).

O material em estudo proveniente da Bolívia, e depositado nas coleções do Museo de Historia Natural Noel Kempff Mercado (MNKM), Yacimientos Petrolíferos 
Fiscales Bolivianos (YPFB) e American Museum of Natural History (AMNH), foi coletado na região de La Paz, Cochabamba e Chuquisaca. Estratigraficamente, estas amostras são das Formações Icla e Bélen (Emsiano ao Eifeliano/Givetiano, Isaacson \& Díaz Martínez, 1995), e Sica Sica (Eifeliano/Givetiano ao Eo-Frasniano, Isaacson \& Díaz Martínez, 1995).

\subsection{Preparação dos Fósseis}

O processo de preparação dos fósseis envolveu a seleção de amostras com características morfológicas adequadas para um estudo sistemático. Uma vez selecionados, os fósseis foram analisados e fotografados com uma câmera digital SONY ${ }^{\circledR}$ Cyber-shot. Alguns fósseis pertencentes à FUMDHAM foram submetidos à técnica do óxido de magnésio em fita, visando realçar a morfologia das amostras. A técnica, discriminada em Carvalho (1995: 16), consiste em impregnar as amostras com o vapor desprendido do óxido de magnésio em fita. Antes da impregnação, os espécimes são recobertos com nanquim preto.

Após a etapa de revisão taxonômica, a relação de táxons identificada em cada unidade geológica de cada bacia sedimentar foi comparada com grupos de fósseis presentes em outras localidades, buscando correspondência de táxons em cada lugar.

\subsection{Descrição das Amostras}

Para a revisão do material, foi adotada a terminologia morfológica mais recente do Treatise on Invertebrate Paleontology, Part $O$ (Whittington et al., 1997). Adicionalmente, descrições de trilobitas Phacopida encontradas em Eldredge \& Braniša (1980), Cooper (1982), Lieberman et al. (1991), Lieberman (1993), Carvalho et al. (1997), Sandford (2005), Carvalho (2006), Soares (2007), Soares et al. (2008b) e Simões et al. (2009), também foram utilizadas. Os caracteres morfológicos passíveis de alteração tafonônomica considerados foram, para Calmoniidae, a forma e o grau de inflação do lobo glabelar, profundidade dos sulcos axiais e glabelares e presença ou ausência (ou mesmo o grau de definição) de ornamentação externa (Soares, 2007; Soares et al., 2008a; Mori, 2013); para Homalonotidae, foram consideradas a forma do 
lobo glabelar e a presença ou ausência de ornamentação externa (Soares, 2007; Soares et al., 2008a; Simões et al., 2009).

Para análise da morfologia, foram priorizados o céfalo e o pigídio das amostras, por serem as partes do animal com maior riqueza de informações e por serem mais comumente encontradas nas coleções científicas.

\section{CONTEXTO GEOLÓGICO}

Neste tópico, será descrita a geologia das bacias do Amazonas e Parnaíba, com ênfase nas unidades litoestratigráficas devonianas em que os fósseis do estudo se encontram: formações Pimenteira e Longá (Bacia do Parnaíba) e formações Maecuru e Ererê (Bacia do Amazonas) (Fig. 2, 3). 


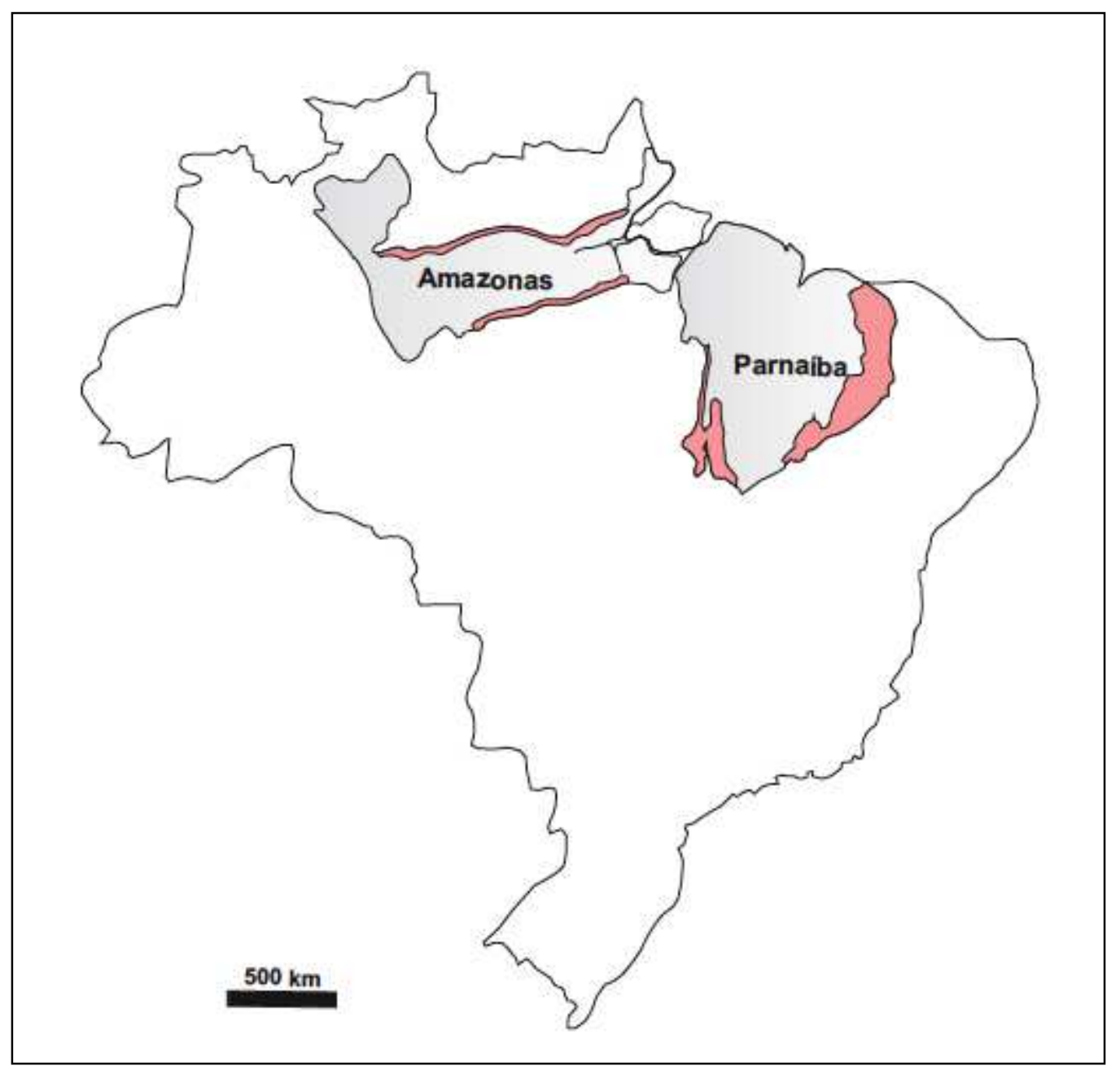

Figura 2: Localização das faixas de afloramento de rochas sedimentares précarboníferas nas bacias do Amazonas e Parnaíba. Modificado de Schobbenhaus \& Brito Neves (2003). 


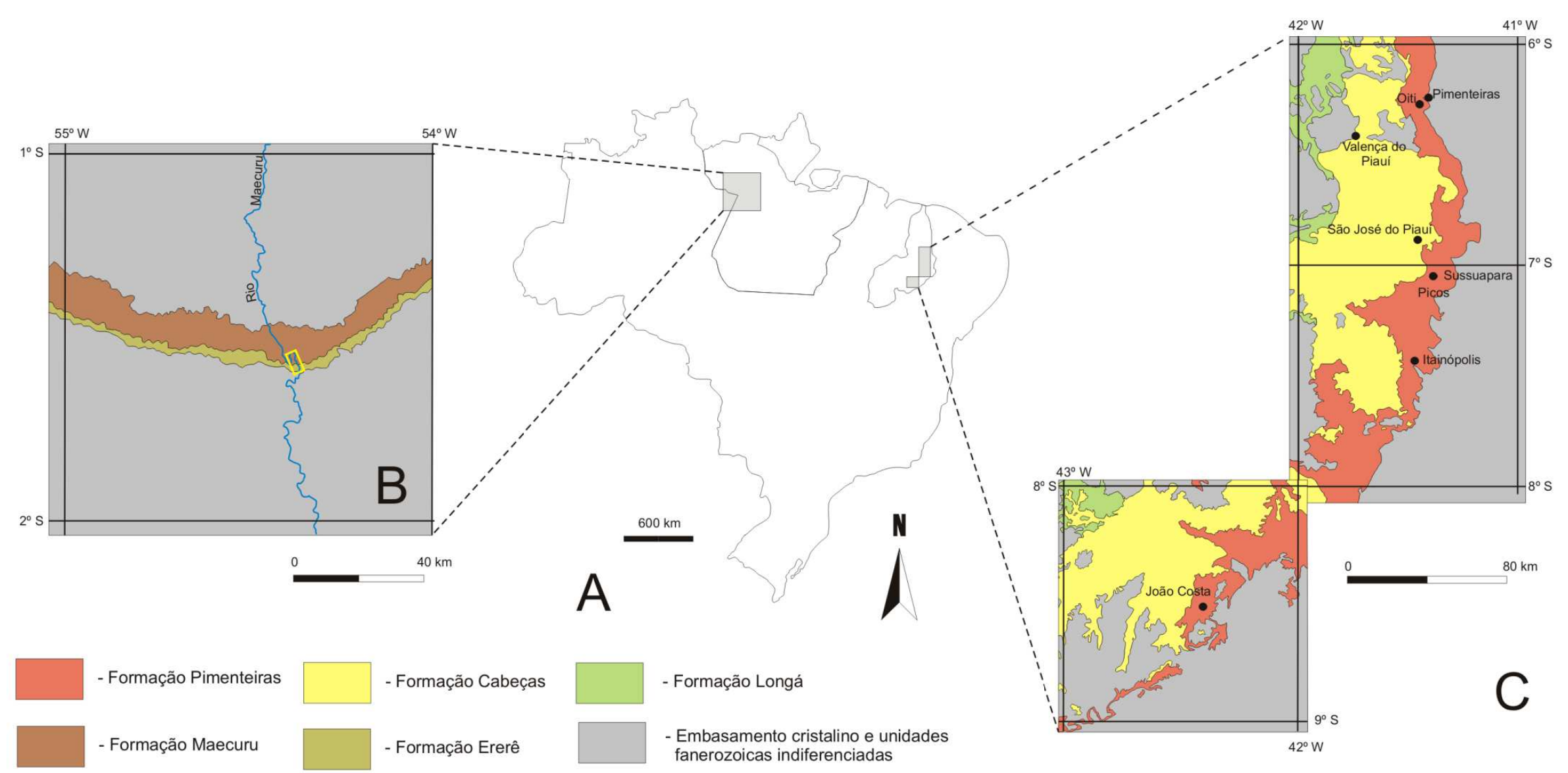

Figura 3: Locais onde trilobitas Homalonotidae e Calmoniidae foram encontrados, nas bacias do Amazonas (retângulo em B, segundo ur Silva \& Fonseca, 2005; Ponciano \& Machado, 2007; e Fonseca \& Ponciano, 2011) e do Parnaíba (afloramentos em municípios apresentados em C, segundo Carvalho, 1995; e Leme et al., 2011). Modificado de Faria et al. (2004) (B), e de Angelim et al. (2004) e Vasconcelos et al. (2004) (C). 


\subsection{Bacia do Parnaíba}

Esta bacia corresponde à porção centro-sul da Província Sedimentar do Meio Norte (Góes, 1995), também conhecida como Província Parnaíba (Almeida et al., 1977). A província é limitada, a norte, pelo Arco Vicente Ferrer-Urbano Santos-Guamá; a leste, pela Falha de Tauá; a sudeste, pelo Lineamento de Senador Pompeu; a oeste, pelo Lineamento Tocantins-Araguaia; e a noroeste, pelo Arco do Tocantins (Góes, 1995).

Ainda na fase de rifteamento da bacia, durante o Cambriano - Ordoviciano houve a deposição de siliciclastos do Grupo Jaibaras (Vaz et al., 2007). Na fase de sinéclise, os depósitos são representados pelos grupos Serra Grande (Siluriano), Canindé (Mesodevoniano - Eocarbonífero) e Balsas (Neocarbonífero - Eotriássico) (Góes \& Feijó, 1994), além das formações Pastos Bons (Meso a Neojurássico), Corda, Grajaú, Codó (Neoaptiano - Eoalbiano) e Itapecuru (Mesoalbiano - Neocretáceo) (Rossetti et al., 2001; Rezende, 2002; Vaz et al., 2007).

A Formação Pimenteira, compreendida entre o Neoeifeliano e o Eogivetiano (Melo, 1985; Grahn et al., 2008), está inserida no Grupo Canindé (Fig. 4), em contato concordante com as formações Itaim, sotoposta, e Cabeças, sobreposta (Carvalho, 1995). Sua litologia consiste em ritmitos de arenitos finos com estratificação cruzada hummocky e pelitos bioturbados (Vaz et al., 2007; Fonseca \& Ponciano, 2011), representado ambientes de plataforma rasa dominada por tempestades (Della Fávera, 1990). A variedade fóssil encontrada consiste de invertebrados diversos (braquiópodes, pelecípodes, gastrópodes, tentaculídeos, trilobitas, crinoides, conularídeos, ostracodes e hiolitídeos) vertebrados (condrictes e acantódios), e fragmentos de vegetais (Kegel, 1953; Fonseca \& Melo, 1987; Melo, 1985; Carvalho, 1995; Caputo et al., 2005). Segundo Melo (1988), na faixa de afloramentos localizada a leste da bacia, os horizontes fossilíferos ocorrem principalmente na base de arenitos com estratificação cruzada hummocky, associados a intraclastos de argila ou seixos de quartzo, e no interior de concreções ferruginosas, localizadas na parte superior da seção aflorante.

O Membro Passagem, de idade eogivetiana, em arenitos de coloração amarelada a arroxeada, muito micáceos e de granulometria fina, com intercalações de siltitos e arenitos conglomeráticos (Fonseca \& Ponciano, 2011). A fauna é menos diversificada 
que a da fácies mais argilosa da Formação Pimenteira, havendo, no entanto, o registro de algumas novas formas (Melo, 1988). Os fósseis consistem de invertebrados (braquiópodes, pelecípodes, gastrópodes, trilobitas, tentaculídeos e crinoides) e fragmentos vegetais (Ponciano et al., 2010).

Sobreposta à Formação Cabeças, em contato concordante, está a Formação Longá, caracterizada por folhelhos escuros, homogêneos ou laminados, com bioturbações, intercalados a arenitos finos com fácies tempestíticas (Lima \& Leite, 1978; Melo, 1988; Della Fávera, 1990). A idade da formação, segundo Quadros (1982), está compreendida entre o Fameniano e o Tournaisiano. Kegel (1953) e Melo (1988) reportam a ocorrência de macrofósseis invertebrados na porção basal desta unidade, em uma localidade próxima a cidade de Valença do Piauí, no Estado do Piauí. Os fósseis correspondem a pelecípodes, braquiópodes, trilobitas, ostracodes e possivelmente tentaculitídeos. Já no topo desta unidade, de idade tournaisiana, ocorrem braquiópodes linguliformes, equinodermos, e restos de plantas e escamas de peixes (Duarte, 1936).

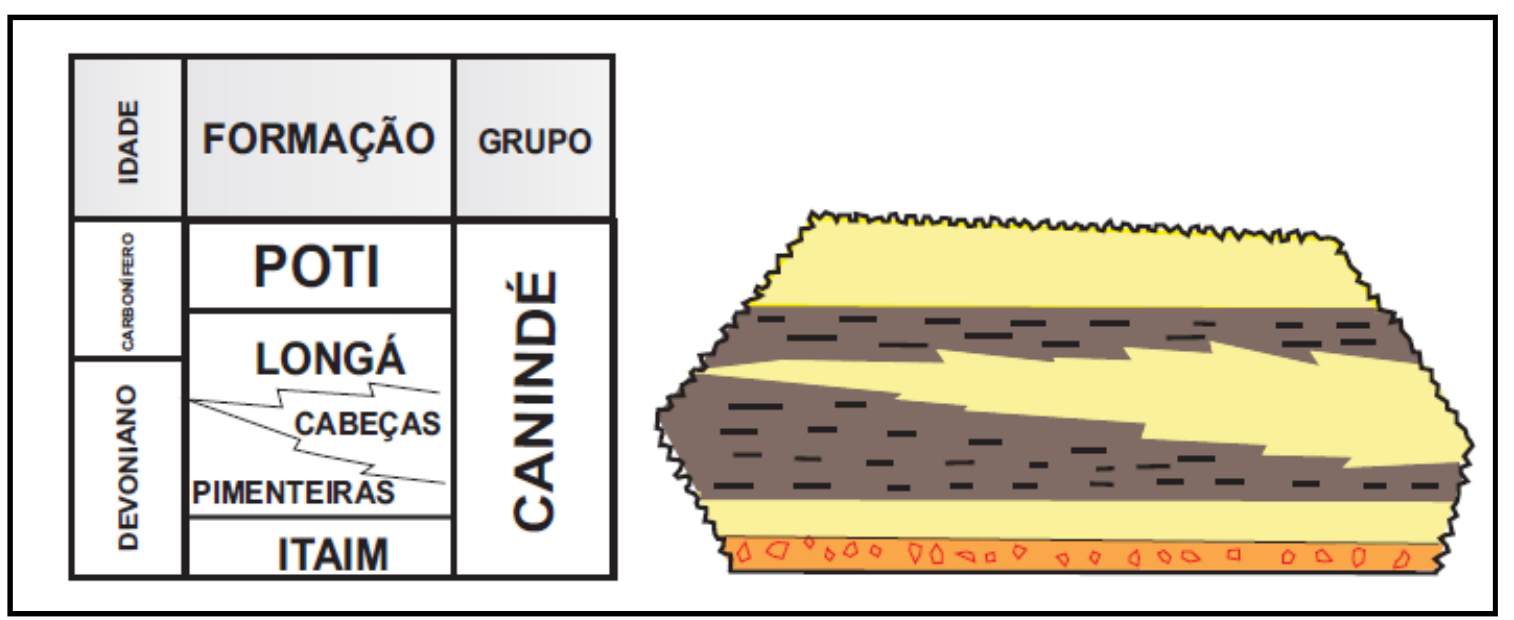

Figura 4: Seção estratigráfica do Grupo Canindé. Extraído de Góes (1995).

\subsection{Bacia do Amazonas}

A Bacia do Amazonas limita-se, ao norte e ao sul, com os crátons das Guianas e Brasileiro, respectivamente; a oeste, está separada da Bacia do Solimões através do Arco de Purus, e a leste, da Bacia do Marajó, pelo Arco de Gurupá (Cunha, 2000). As formações Prosperança e Acari, reunidas no Grupo Purus, correspondem a depósitos 
localizados, cuja gênese é anterior à efetiva implantação da sinéclise (Cunha et al., 2007).

Os depósitos na bacia correspondem aos grupos Trombetas (Neordoviciano Lochkoviano/Eoemsiano), Urupadi (Neoemsiano - Eogivetiano), Curuá (Eofrasniano Mesotournaisiano), Formação Faro (Neoviseano), e grupos Tapajós (Neobashkiriano Neopermiano), e Javari (Aptiano - Mioceno/Plioceno). Dados bioestratigráficos a partir de palinomorfos (Playford \& Dino, 2000; Melo \& Loboziak, 2003; Grahn \& Melo, 2004; Grahn, 2005) e conodontes (Nascimento et al., 2006) permitiram o estabelecimento das idades.

O Devoniano da Bacia do Amazonas é representado pelo Grupo Urupadi (Caputo, 1984), que é composto pelas formações Maecuru e Ererê. Cunha et al. (2007) determinaram como seção-tipo deste grupo o intervalo entre 1.319 e $1.450 \mathrm{~m}$ do poço 1AM-1-AM (03 17' 06" S / 59 52' 24" W; Fig. 2.4). A Formação Maecuru, de idade neoemsiana a neoeifeliana (Melo \& Loboziak, 2003; Grahn \& Melo, 2004), foi primeiramente reconhecida por Derby (1878), a partir de siliciclastos aflorantes no Rio Maecuru, no Estado do Amazonas. É composta principalmente por arenitos, com raras intercalações de argilitos e siltitos, associados a ambientes flúvio-deltaicos a neríticos (Cunha et al., 2007). Esta unidade contém abundantes fósseis de invertebrados marinhos, incluindo trilobitas, braquiópodes, crinoides, conularídeos, corais, tentaculitídeos, briozoários, gastrópodes e belerofontídeos (Ponciano \& Machado, 2007; Fonseca \& Ponciano, 2011), provenientes do topo da unidade (Melo, 1988).

Na Formação Ererê (Neoeifeliano - Eogivetiano, Melo \& Loboziak, 2003; Grahn \& Melo, 2004), sobreposta à Maecuru, predominam sedimentos mais finos (arenitos finos, siltitos e argilitos), neríticos, refletindo uma transgressão na bacia (Cunha et al., 2007), cujo máximo situa-se pouco acima do limite Eifeliano / Givetiano (Melo \& Loboziak, 2003). A fauna fóssil, presente na base da unidade, é composta por trilobitas, braquiópodes, pelecípodes, gastrópodes, tentaculitídeos e crinoides (Melo, 1988). Segundo Melo (1988), embora os fósseis nesta formação sejam menos diversificados do que aqueles observados na unidade sotoposta, sua ocorrência abrange maior variedade litológica. 


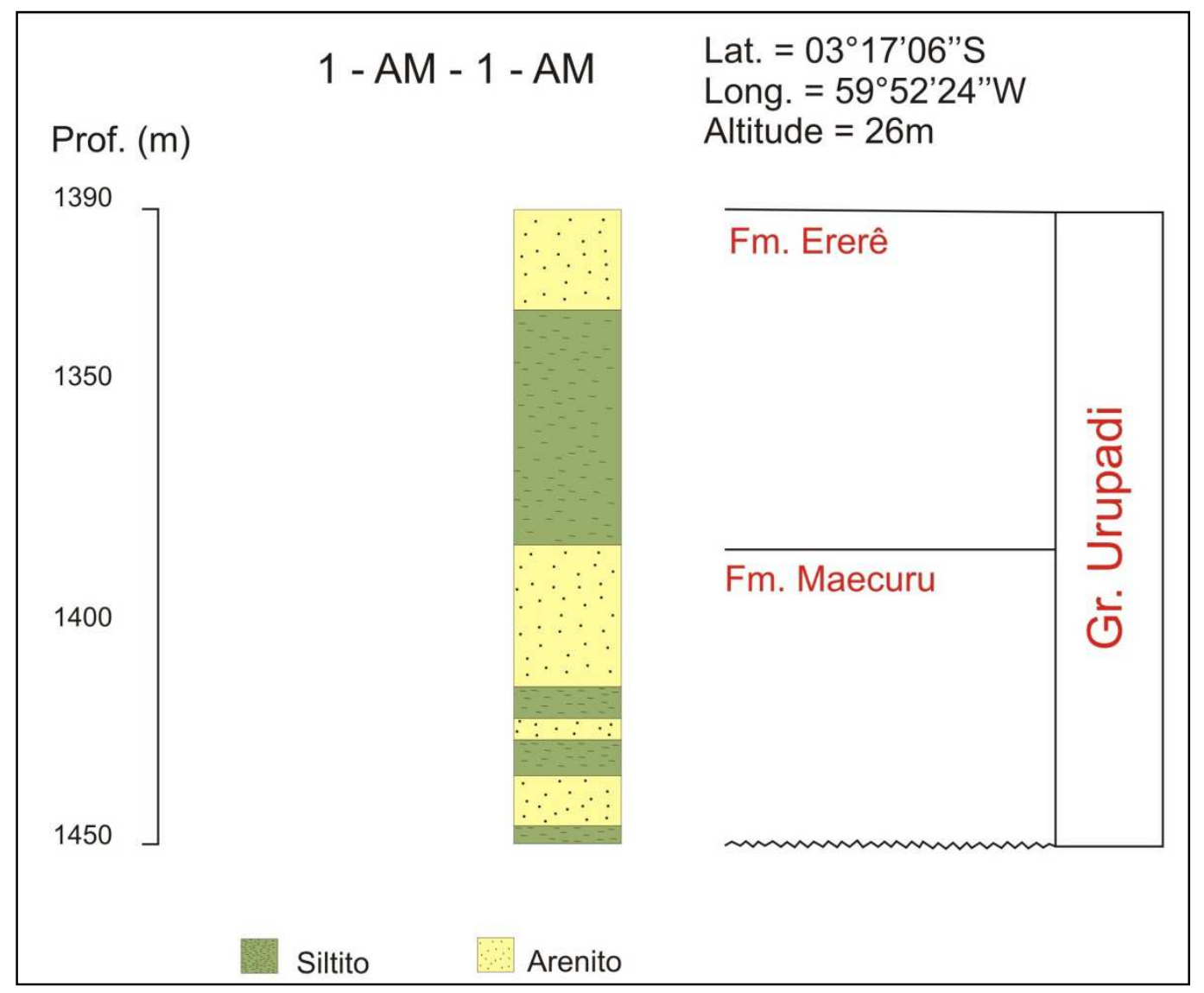

Figura 5: Seção-tipo do Grupo Urupadi. Extraído de Cunha et al. (2007).

\section{RESULTADOS}

\subsection{Revisão Sistemática (Anexos II, III, IV, V e VI).}

A partir da análise dos exemplares de trilobitas observados nas coleções Nacionais (MCT/DNPM, IGEO/UFRJ e MPEG) e internacionais (American Museum of Natural History- AMNH, Museo de Historia Natural Noel Kempff Mercado - MNKM e Yacimientos Petrolíferos Fiscales Bolivianos - YPFB) foi possível desenvolver cinco artigos científicos, a saber: (a) The "Metacryphaeus tuberculatus group" (trilobita, calmoniidae) from the devonian of the Parnaíba basin, Brazil" (in press) (vide Anexo Anexo II), (b) Metacryphaeus tuberculatus (Kozlowski, 1923) and M. australis (Clarke, 1913) (Trilobita, Phacopida) from the Devonian of Paraná Basin: taxonomy and palaeobiogeography, que foi submetido ao periódico Ameghiniana (vide Anexo III), (c) Systematic revision and paleobiogeography of calmoniid trilobites (Phacopida) from the Devonian of the Amazon Basin, Brazil (vide Anexo IV), (d) The genus Burmeisteria 
Salter, 1865 (Trilobita, Homalonotidae) in the Devonian of the Parnaíba Basin, Brazil (em preparação) (vide Anexo V) e (e) The occurrence of Phacopida trilobites from Pimenteira Formation at João Costa, Piauí, Brazil. Geologia USP - Série Científica (vide Anexo VI).

Resumo do artigo: The "Metacryphaeus tuberculatus group" (trilobita, calmoniidae) from the devonian of the Parnaíba basin, Brazil (ANEXO II).

A sistemática dos trilobitas calmonídeos do Devoniano da Bacia do Parnaíba, nordeste do Brasil, foi revisada neste estudo, considerando os trabalhos recentes sobre esta família no Brasil, os quais usam como critério as modificações tafonômicas e como estas afetam a taxonomia. O presente estudo reforça a ocorrência de Metacryphaeus tuberculatus Kozlowski, 1923, na Formação Pimenteira com base em espécimes encontrados na região da cidade de João Costa, sudeste do Estado do Piauí. As espécies Metacryphaeus meloi Carvalho et al., 1997, da Formação Cabeças é sinonimizada com M. tuberculatus com base na similaridade entre este e o táxon boliviano, e alguns dos caracteres utilizados para distingui-los foram interpretados como de origem tafonômica. O estudo também apresenta evidência de variação intraespecífica em $M$. tuberculatus da Formação Cabeças. Estes resultados levantam questões importantes sobre a relação estratigráfica entre a Formação Pimenteira e Cabeças e amplia a distribuição de $M$. tuberculatus. Por fim, a revisão de algumas espécies incluídas no Grupo "Metacryphaeus tuberculatus" é considerado aconselhável.

O texto completo do artigo, com os resultados e discussões, é apresentado no Anexo II.

Resumo do artigo: Metacryphaeus tuberculatus (Kozlowski, 1923) and M. australis (Clarke, 1913) (Trilobita, Phacopida) from the Devonian of Paraná Basin: taxonomy and palaeobiogeography.

Os trilobitas calmonídeos do gênero Metacryphaeus incluem M. tuberculatus, $M$. kegeli, M. meloi, M. rotundatus, M. giganteus, M. convxus, M. curvigena, M. branisai, 
M. caffer, M. australis e M. allardyceae. A distribuição geográfica desse gênero engloba diversas áreas do Gondwana tais como Bolívia, Peru, Brasil, Ilhas Falkland e África do Sul. O presente estudo reporta uma nova ocorrência de M. tuberculatus na Bacia do Paraná (estados de Goiás e Paraná, Brasil), nas subbacias Alto Garças e Apucarana. O estudo comparou M. tuberculatus com todas as espécies do gênero Metacryphaeus, especialmente com M. australis, o qual apresenta uma nova ocorrência na Bacia do Paraná. Estas novas ocorrências de M. tuberculatus implicam em uma interpretação acerca da dispersão da espécie diferente do que consta na literatura. Aparentemente, esta espécie se originou no Eodevoniano e viveu na Bolívia, Peru e na Subbacia Apucarana (Bacia do Paraná, Brasil). Provavelmente a dispersão entre essas áreas está relacionada com a transgressão emsiana. Durante o Givetiano, a espécie teria migrado para a Subbacia Alto Garças e para a Bacia do Parnaíba, onde há registros de M. tuberculatus. Estas evidências indicam uma interpretação paleobiogeográfica diferente daquela presente para o braquiópode Tropidoleptus e o equinodermo Exaesiodiscus no Gondwana, sugerindo um padrão de migração diferente para o Devoniano do Brasil.

O texto completo do artigo, com os resultados e discussões, é apresentado no Anexo III.

Resumo do artigo: Systematic revision and paleobiogeography of calmoniid trilobites (Phacopida) from the Devonian of the Amazon Basin, Brazil

Uma revisão sistemática de alguns trilobitas do Devoniano (eifeliano) da Bacia do Amazonasé aqui apresentada. Os resultados mostram que estes trilobitas, originalmente associados com os gêneros Phacops Emmrich, 1839, Dalmanites Barrande, 1852 e Homalonotus König, 1825 consistem em Calmoniidae atribuídos aos gêneros Malvinella Wolfart, 1968 e Pennaia Clarke, 1913, este último com dúvidas. Estes resultados corroboram revisões anteriores que incluem trilobitas do Devoniano amazônico em táxons bolivianos. Rotas de migração são discutidas com base nas últimas revisões sistemáticas desta família de trilobitas na Bolívia e na Bacia do Amazonas.

O texto completo do artigo, com os resultados e discussões, é apresentado no Anexo IV. 
Resumo do artigo: The genus Burmeisteria Salter, 1865 (Trilobita, Homalonotidae) in the Devonian of the Parnaíba Basin, Brazil

O conhecimento acerca dos trilobitas homalonotídeos no Brasil é relativamente pobre quando comparada a outros invertebrados marinhos fósseis. O gênero melhor documentado desta família é Burmeisteria Salter, 1856, do Eodevoniano da Bacia do Paraná e Mesodevoniano da Bacia do Parnaíba, onde a espécie B. notica Clarke, 1913 era conhecida como a única representante por quase um século desde a clássica monografia de Clarke (1913). Trabalhos recentes focando a revisão de invertebrados fósseis sob a perspectiva da Tafonomia (Simões et al., 2009) concluíram que, ao menos na Bacia do Paraná, o gênero Burmeisteria também estaria representado por uma segunda espécie, B. herscheli Murchison, 1839. O presente estudo faz algumas reflexões sobre a atual sistemática das espécies de Burmeisteria nas bacias brasileiras, com base na análise de fósseis de homalonotídeos da Formação Pimenteira na Bacia do Parnaíba, Brasil.

O texto completo do artigo, com os resultados e discussões, é apresentado no Anexo V.

Resumo do artigo: The occurrence of Phacopida trilobites from Pimenteira Formation at João Costa, Piauí, Brazil.

Os fósseis de macroinvertebrados marinhos do Devoniano da Bacia do Parnaíba estão entre os mais diversos do Paleozoico do Brasil. Nos últimos anos, a maioria das revisões sobre estes fósseis envolveu trilobitas das formações Pimenteira e Cabeças, na região das cidades de Picos e Pimenteiras, no Estado do Piauí. Esta pesquisa relata a ocorrência de trilobitas devonianos da Formação Pimenteira, aflorante na cidade de João Costa, na região do Parque Nacional da Serra da Capivara, no sudeste do Piauí, onde duas espécies foram reconhecidas: Burmeisteria notica Clarke, 1913, e Metacryphaeus cf. australis Clarke, 1913. A ampla ocorrência destes gêneros no Domínio Malvinocáfrico mostra que eventos transgressivos durante parte do Devoniano favoreceram a comunicação entre as faunas das províncias Andina, Brasileira e Sulafricana, através da redução das barreiras geográficas. Finalmente, apesar do caráter 
ainda preliminar dos estudos sobre trilobitas da Formação Pimenteira, esta unidade continua fornecendo novos dados paleontológicos que contribuem para o entendimento destas assembleias fósseis.

O texto completo do artigo, com os resultados e discussões, é apresentado no Anexo VI. 


\section{DISCUSSÃO}

\subsection{Paleobiogeografia}

O Eo e Mesodevoniano são marcados pela presença de três grandes unidades biogeográficas no globo: o Domínio do Velho Mundo, o Domínio das Américas Orientais e o Domínio Malvinocáfrico (Fig. 6). Enquanto as duas primeiras áreas correspondem à fragmentação, no Devoniano, do Domínio Siluriano do Norte, caracterizado por depósitos carbonáticos indicativos de clima quente, o Domínio Malvinocáfrico já estava presente desde o Siluriano (Boucot, 1988).

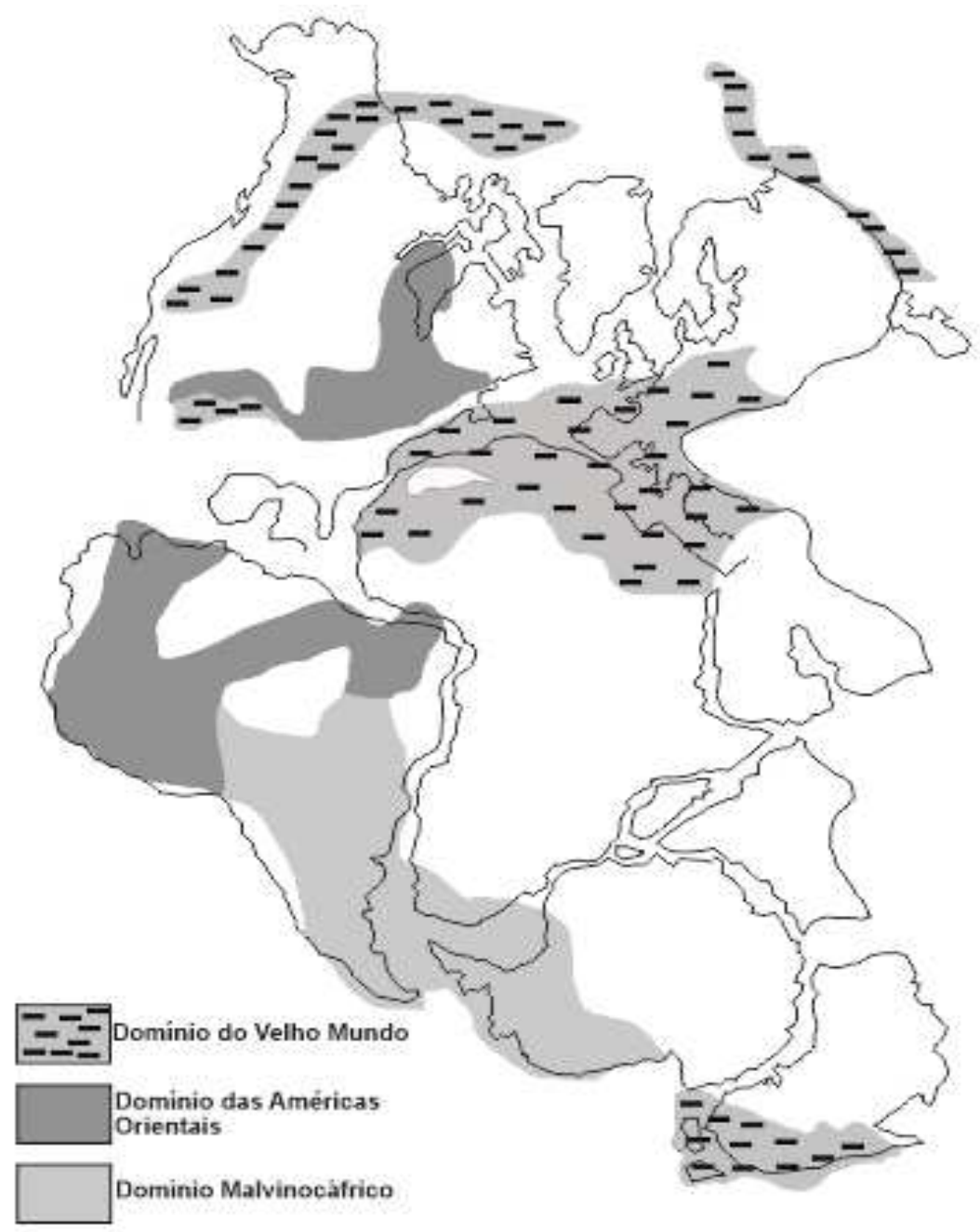

Figura 6: Mapa paleogeográfico do Devoniano, mostrando os domínios paleobiogeográficos. Modificado de www.palaeos.com. 
O Domínio do Velho Mundo abrangia áreas do noroeste da América do Norte, Europa, Ásia, norte da África, Austrália e Nova Zelândia. Tal província caracterizava-se pela presença de rochas carbonáticas e elementos faunísticos típicos de mares mais quentes. (Boucot, 1988).

As regiões centro-leste e sul-sudoeste da América do Norte, mais o norte da América do Sul são englobadas no Domínio das Américas Orientais, a qual se divide nas seguintes subprovíncias: Appohimchi, Nevada, Michigan Basin-Hudson Bay, Colombiana e Amazônica. Boucot et al. (1969) inicialmente englobaram as duas últimas subprovíncias em uma, a qual denominaram Amazônica-Colombiana. Contudo, o peculiar intercâmbio faunístico encontrado nas bacias do Amazonas e Parnaíba, caracterizado por braquiópodes típicos das Américas Orientais e trilobitas característicos do Domínio Malvinocáfrico, levou Boucot (1988) a propor uma subprovíncia dentro das Américas Orientais apenas para estas localidades, a Subprovíncia Amazônica.

O Domínio Malvinocáfrico, que inicialmente compreendia apenas as faunas da Bacia do Paraná, Ilhas Falklands e África do Sul (Clarke, 1913), foi ampliado para compreender mais localidades, com base nas afinidades dos fósseis. Eldredge \& Ormiston (1979) reconheceram três províncias dentro deste domínio: Brasileira, incluindo as bacias paleozoicas do Brasil e possivelmente o Uruguai; Andina, contendo a Argentina, Bolívia, Peru e Paraguai; e Malvino-Sul Africana, composta pelas Ilhas Falkland e África do Sul, e possivelmente Antártica. Estes autores basearam-se nos trilobitas, especialmente da família Calmoniidae, para estabeler os limites do Domínio Malvinocáfrico. Os Calmoniidae são considerados endêmicos do Domínio Malvinocáfrico (Eldredge \& Ormiston, 1979). Nessa concepção, as bacias do Amazonas e Parnaíba estão incluídas na Província Brasileira do Domínio Malvinocáfrico, uma vez que ambas apresentam táxons de Calmoniidae. Por outro lado, estudos focados na paleobiogeografia de braquiópodes demonstraram maior afinidade deste grupo com faunas dos domínios das Américas Orientais e Velho Mundo (Boucot et al., 1969; Boucot, 1988), conforme mencionado acima. Trabalhos mais recentes (e.g. Melo, 1988; Carvalho \& Fonseca, 2007) tratam ambas as bacias como limites de transição entre os três domínios. Cabe ressaltar que o argumento acerca do endemismo dos Calmoniidae de Eldredge \& Ormiston (1979) pode não se sustentar mais, visto que recentemente 
foram descobertos fósseis de Calmoniidae em estratos do Neoemsiano da parte basal da Formação Floresta, na Colômbia (Morzadec et al., 2015), localidade esta que faz parte da Subprovíncia Colombiana do Domínio das Américas Orientais (Boucot, 1988). O intercâmbio de faunas marinhas nas bacias do Parnaíba e Amazonas pode estar relacionado à posição intermediária destas bacias em relação aos três domínios paleobiogeográficos; também pode estar relacionado à época de deposição das formações devonianas portadoras desses fósseis nas duas bacias (Maecuru e Ererê na Bacia do Amazonas; Pimenteira e Membro Passagem na Bacia do Parnaíba), correspondente ao intervalo Eifeliano-Givetiano (Mesodevoniano), quando o endemismo das faunas marinhas já não era tão acentuado, como observado no Eodevoniano (Fonseca, 2015).

Apesar da afinidade com faunas das Américas Orientais e Velho Mundo, a assembleia fóssil nas bacias do Parnaíba e Amazonas caracteriza-se pela baixa diversidade de espécies e de táxons supraespecíficos, quase total ausência de corais e briozoários, e ausência de conodontes, braquiópodes estromatoporóides, algas calcáreas e graptólitos (Boucot, 1988), tal qual observado nas demais localidades malvinocáfricas.

O estabelecimento dos três domínios paleobiogeográficos é evidente no início do Devoniano, quando eventos regressivos que já atuavam no Siluriano tornaram o provincialismo mais conspícuo. Este reconhecimento é observado até o limite EifelianoGivetiano, quando as transgressões características do Devoniano, iniciadas já no Emsiano, favoreceram a disseminação de espécies cosmopolitas. $O$ auge do cosmopolitanismo foi ao intervalo Frasniano-Fammeniano (Figs. 7 e 8) (Boucot, 1988). 


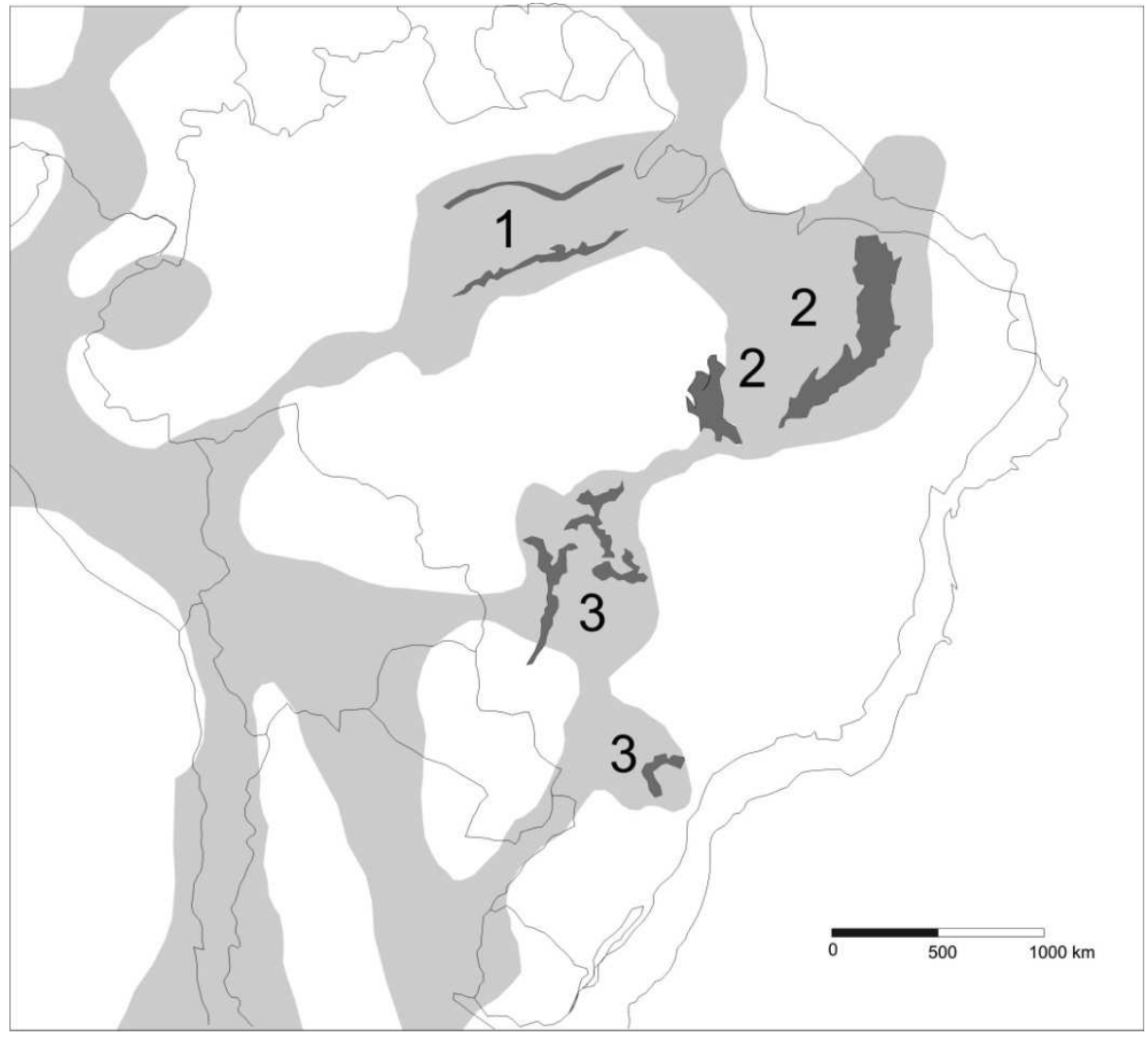

Figura 7: Reconstrução paleogeográfica do Brasil e áreas adjacentes no intervalo Neoemsiano-Eifeliano (final do Eodevoniano-início do Mesodevoniano), mostrando os cinturões de afloramento das três principais bacias paleozoicas brasileiras: Bacia do Amazonas (1), Bacia do Parnaíba (2) e Bacia do Paraná (3). Modificado de Melo (1988). 


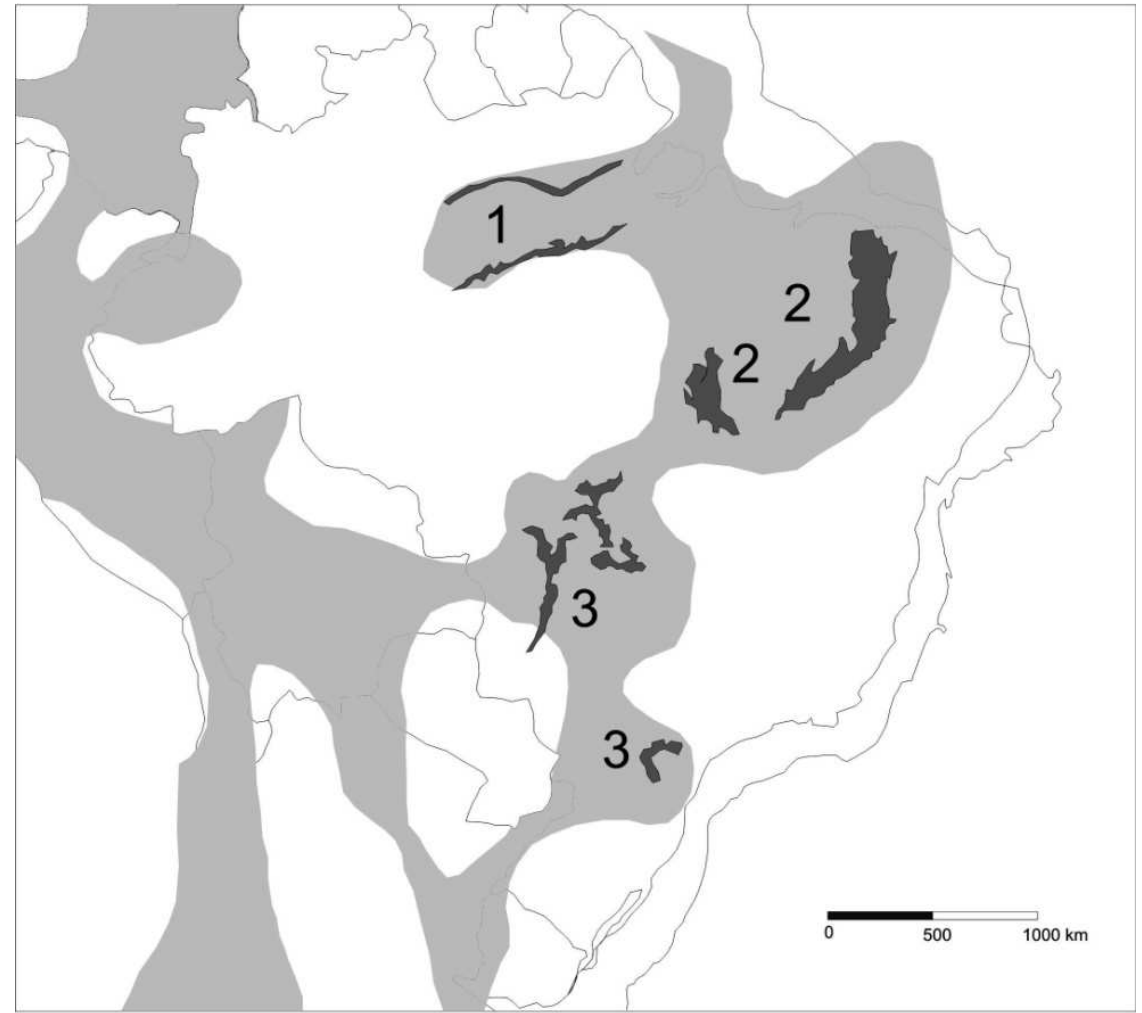

Figura 8: Reconstrução paleogeográfica do Brasil e áreas adjacentes no intervalo Neoeifeliano-Givetiano (Mesodevoniano). Indicações das bacias paleozoicas é a mesma da figura anterior. Modificado de Melo (1988).

Eventos transgressivos-regressivos caracterizaram o Devoniano, tendo atingido seu apogeu no limite Eifeliano-Givetiano, caracterizado por um evento de extinção denominado KAČÁK. No Brasil, evidências do evento KAČÁK estão presentes em alguns sítios de idade neoeifeliana/eogivetiana da Bacia do Paraná. Nestas localidades, fósseis de invertebrados marinhos apresentam redução drástica de tamanho, além de uma redução na diversidade e presença de elementos típicos de outros domínios paleobiogeográficos (Bosetti et al., 2011). Segundo Bosetti et al. (2011), as características desta assembleia fóssil indicam o declínio na fauna malvinocáfrica, sendo os elementos desta assembleia remanentes da típica fauna malvinocáfrica.

$\mathrm{Na}$ Bacia do Amazonas, os trilobitas são representados principalmente pela família Calmoniidae, relacionados ao "Grupo Malvinella" de Lieberman et al. (1991), com estreita relação com gêneros da Bolívia (Eldredge \& Ormiston, 1979). Além desta família, também estão presentes representantes Homalonotidae e Synphoriidae. No 
presente trabalho (ver Anexo IV), a revisão do material da Bacia do Amazonas levou à constatação de mais materiais associados ao gênero Malvinella, corroborando estudos anteriores que vinham relacionando fósseis a este gênero (e.g. Lieberman et al., 1991; Carvalho \& Ponciano, 2015). A suspeita da presença de Calmoniidae basais na bacia, levantada por Eldredge \& Ormiston (1979), foi retomada no presente estudo, a partir da comparação com fósseis bolivianos.

$\mathrm{Na}$ Bacia do Parnaíba, os Calmoniidae estão relacionados ao "Grupo Metacryphaeus" de Lieberman (1993), além da espécie de Homalonotidae Burmeisteria notica, bem difundida no Domínio Malvinocáfrico. A revisão sistemática neste trabalho (ver Anexo II) tratou dos Calmoniidae do gênero Metacryphaeus contidos na Formação Pimenteira. A presença de M. tuberculatus, já reportada por Lieberman et al. (1991) e Lieberman (1993), mas questionada por Carvalho (1995, 1999) e Carvalho et al. (1997), foi confirmada neste estudo. Adicionalmente, a espécie endêmica da Bacia do Parnaíba M. meloi recebeu uma emenda em sua diagnose, a partir da constatação da presença de espinhos genais, até então desconhecidos na espécie. Resultados preliminares acerca dos Homalonotidae na Bacia do Parnaíba sugerem que a localidade abrigava apenas uma espécie, Burmeisteria notica, mas cuja morfologia da glabela assemelha-se àquela indicada por Simões et al. (2009) como típicos da espécie B. herscheli.

\subsection{Rotas migratórias nas bacias do Parnaíba e Amazonas}

Além dos Calmoniidae, a fauna devoniana na Bacia do Amazonas é composta pelos Homalonotidae Digonus derbyi e Burmeisteria oiara (Carvalho \& Ponciano, 2015), pelo Synphoriidae Amazonaspis maecurua (Carvalho \& Fonseca, 2007), e por Dalmanitidae não definidos (Carvalho \& Ponciano, 2015). Segundo Carvalho \& Ponciano (2015), D. derbyi apresenta afinidade com formas do Domínio do Velho Mundo, as quais teriam alcançado a Bacia do Amazonas através de uma passagem localizada a nordeste da bacia, durante a fase transgressiva no Eifeliano. Por outro lado, trilobitas Synphoriidae, aos quais A. maecurua estaria relacionada, teriam migrado até a Bacia do Amazonas por uma passagem a sudoeste. Esta rota a sudoeste também é apontada por Melo (1988) como provável caminho para os Calmoniidae, que teriam origem na Bolívia no Eodevoniano, e durante a transgressão no Eifeliano teriam migrado por esta rota (Fig. 9). Esta hipótese torna-se mais evidente devido à ausência de 
gêneros de trilobitas comuns à Bolívia e à Bacia do Amazonas na Subbacia Alto Garças e na Bacia do Parnaíba.

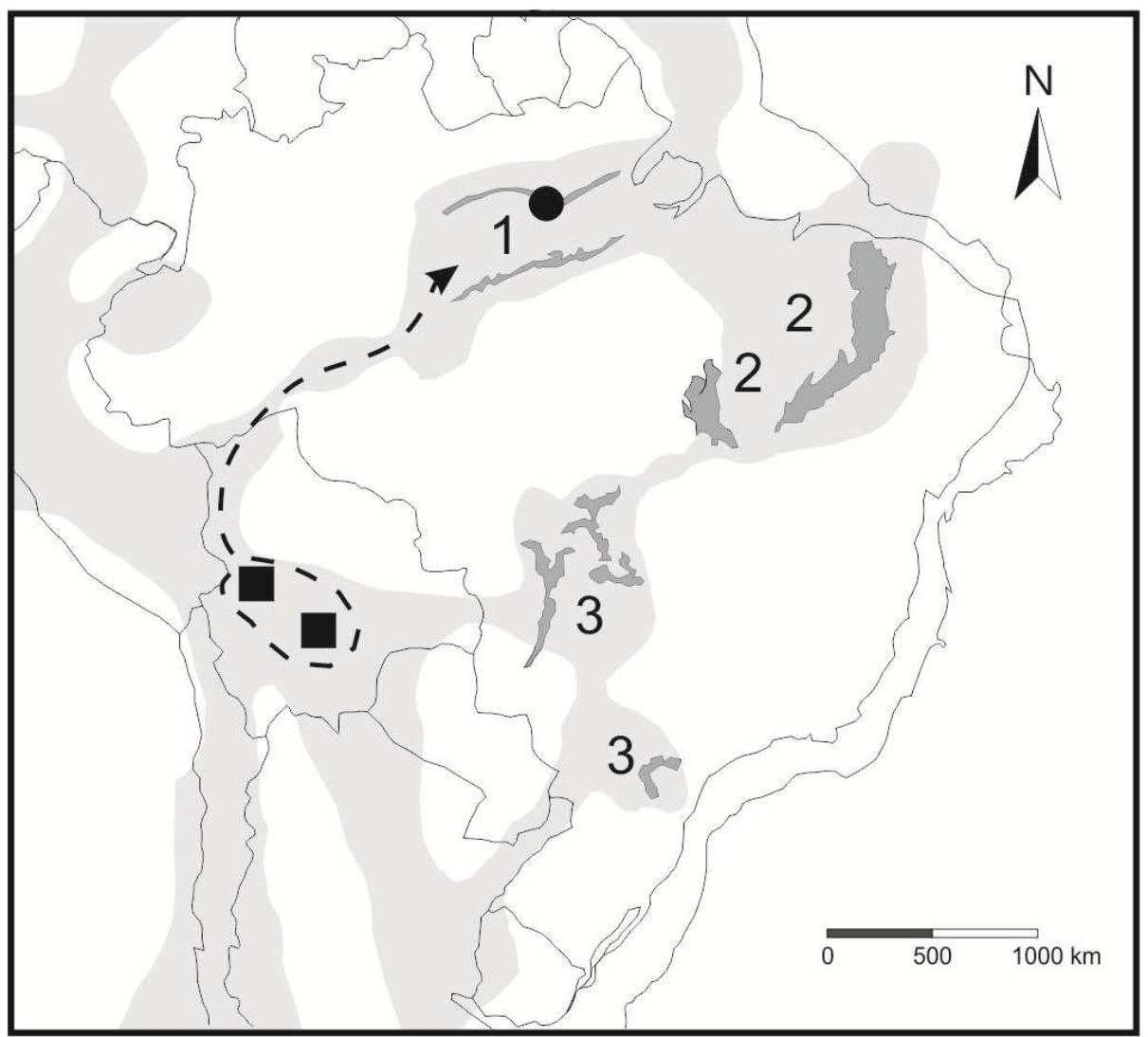

Figura 9: Reconstrução da paleogeografia do Brasil e áreas adjacentes no intervalo Neoemsiano-Eifeliano (final do Eodevoniano-início do Mesodevoniano), mostrando as áreas de afloramentos das três principais bacias sedimentares paleozoicas brasileiras: Bacia do Amazonas (1), Bacia do Parnaíba (2) e Bacia do Paraná (3). Setas indicam o sentido das rotas migratórias de trilobitas Calmoniidae. Quadrados e círculos negros indicam, respectivamente, Calmoniidae do Emsiano da Bolívia e Calmoniidae do Eifeliano da Bacia do Amazonas. Modificado de Melo (1988).

Diferentemente deste cenário, o gênero Eldredgeia, conhecido do Eodevoniano boliviano nas espécies E. venusta (Lieberman, 1993) e E. eocryphaea (Carvalho et al., 2003), ocorre no Eifeliano da Bacia do Parnaíba como E. cf. venusta (Lieberman et al., 1991), e também está presente no Neoeifeliano/Eogivetiano da Bacia do Amazonas como a espécie E. paituna (Carvalho et al., 2003). Além do Brasil, o gênero também está presente no Neoeifeliano do Grupo Bokkevld, na África do Sul (Cooper, 1982). 
Segundo Lieberman et al. (1991), a diferença entre Eldredgeia da Bacia do Parnaíba e E. venusta da Bolívia é sutil, restringindo-se a variações em alguns lobos e sulcos da glabela. A ocorrência de Eldredgeia na Bacia do Parnaíba, podendo ser conespecífica com E.venusta boliviana, indica uma provável rota marinha deste gênero até a Bacia do Amazonas através da Bacia do Parnaíba (Fig. 10). Esta hipótese havia sido sugerida por Melo (1988), ao notar semelhanças entre Eldredgeia (até então associado ao gênero Metacryphaeus) da Bacia do Amazonas com Metacryphaeus do Membro Passagem da Bacia do Parnaíba (M. meloi). Embora hoje se saiba que ambos os fósseis consistem em táxons diferentes, a hipótese ainda é válida dada à ocorrência de E. cf. venusta na Bacia do Parnaíba.

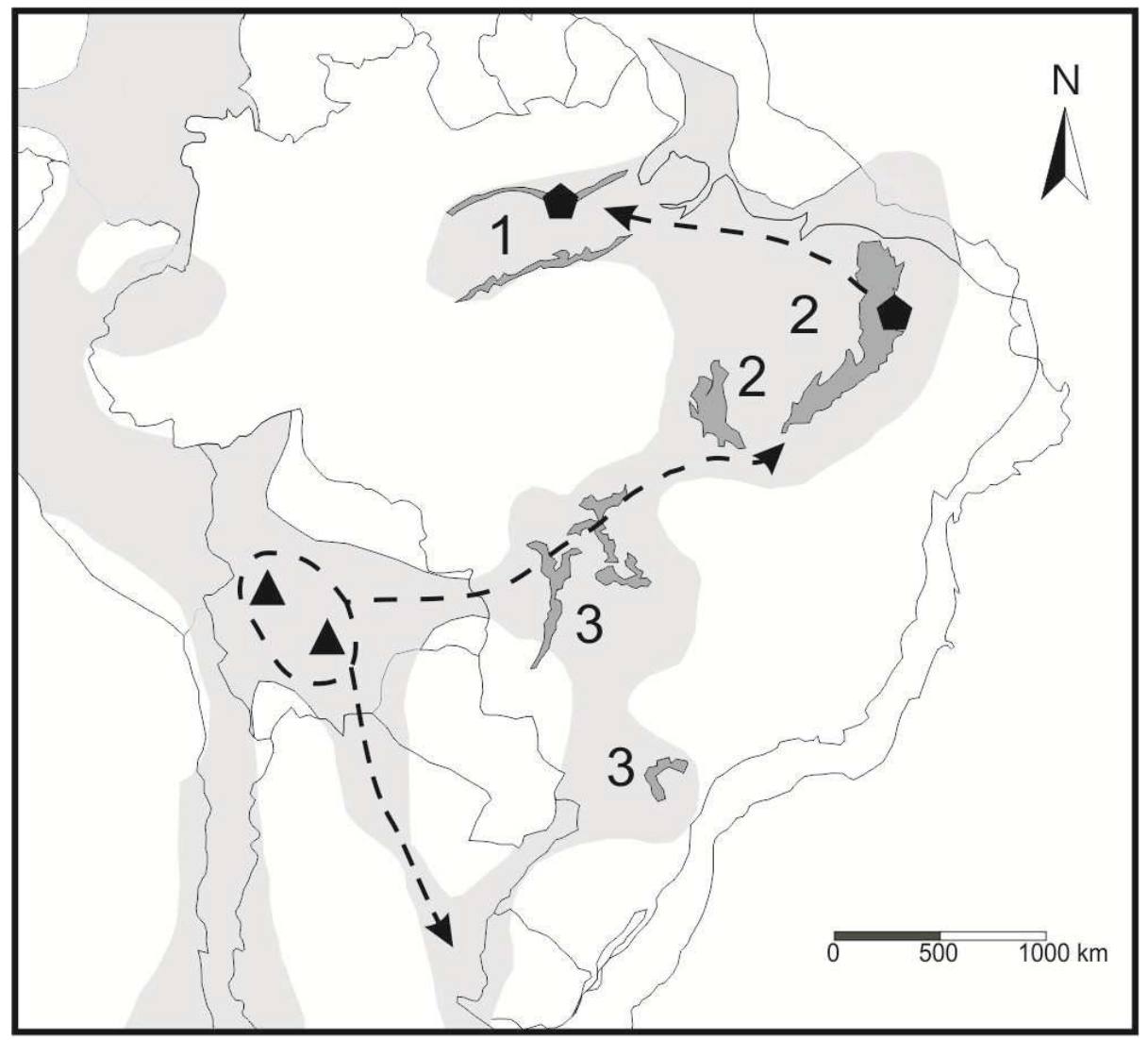

Figura 10: Reconstrução da paleogeografia do Brasil e áreas adjacentes no intervalo Neoeifeliano-Eogivetiano (Mesodevoniano) do Brasil e áreas adjacentes. Setas indicam sentido das rotas migratórias de Calmoniidae do gênero Eldredgeia. Triângulos negros correspondem a ocorrências de Eldredgeia venusta no Emsino da Bolívia. Pentágonos negros representam o gênero Eldredgeia no Neoeifeliano-Eogivetiano das bacias do Parnaíba e Amazonas. Modificado de Melo (1988). 


\subsection{Diversidade de trilobitas}

A Bacia do Amazonas é, sem dúvida, a localidade com maior grau de endemismo dentre as bacias brasileiras, no tocante à diversidade de trilobitas. A Formação Maecuru abriga a maior diversidade e abundância de trilobitas desta bacia, com um total de dez espécies de Calmoniidae (incluindo táxons com nomenclatura em aberto), um Synphoriidae (Amazonaspis maecurua), um Dalmanitidae não identificado e um Homalonotidae (Digonus derbyi). Na Formação Ererê, a diversidade é mais baixa, consistindo de dois Calmoniidae (Eldredgeia paituna e Malvinella cf. tumiloba) e o Homalonotidae Burmeisteria oiara. Todas as espécies conhecidas nas duas formações devonianas são exclusivas da bacia; na maioria dos táxons, o endemismo é específico. $\mathrm{O}$ Homalonotidae Digonus derbyi representa a única ocorrência deste gênero no Brasil, sendo comum em localidades do Domínio do Velho Mundo. Já o gênero Amazonaspis (Synphoriidae) é monoespecífico e exclusivo da Bacia do Amazonas.

Deve-se considerar, contudo, que a sistemática dos trilobitas amazônicos é baseada em material pouco comum e, na maioria dos casos, desarticulado, consistindo de cranídios e pigídios. Por vezes, uma espécie é conhecida a partir de um único espécime desarticulado (e.g. Pennaia? menura e Pennaia? pullina). Dada a natureza do material das formações Maecuru e Ererê, a possibilidade de alguns desses táxons corresponderem a partes de uma mesma espécie deve ser ponderada. Desse modo, a diversidade de trilobitas da Bacia do Amazonas, e em especial da Formação Maecuru, pode ser menor do que suposto.

Comparativamente, a Bacia do Parnaíba apresenta um compartilhamento de táxons maior com a Bacia do Paraná e com a Bolívia, tais como o Homalonotidae Burmeisteria notica (nas três localidades), e os Calmoniidae Metacryphaeus tuberculatus (também nas três localidades) e Eldredgeia (na Bacia do Parnaíba e Bolívia). Em contrapartida, a diversidade de trilobitas na Bacia do Parnaíba é bem menor do que o observado nas demais bacias paleozoicas brasileiras. O Calmoniidae Metacryphaeus meloi é a única espécie endêmica desta localidade. 


\section{CONCLUSÕES}

- A ocorrência de Metacryphaeus tuberculatus na Formação Pimenteira, Eifeliano (Mesodevoniano) da Bacia do Parnaíba, foi confirmada a partir de ocorrências na região do município de João Costa, sudeste do Piauí, corroborando observações de autores prévios (Lieberman et al., 1991; Lieberman, 1993);

- Foi constatada a presença de espinhos genais na espécie Metacryphaeus meloi, presente nos arenitos do Membro Passagem, Eogivetiano (Mesodevoniano) da Formação Pimenteira. Os espinhos assemelham-se aos presentes em M. caffer, ilustrado em Cooper (1982: fig. 61), e consistem em uma característica inédita, não constatada na descrição formal da espécie (Carvalho et al., 1997);

- A presença de M. tuberculatus na Bacia do Parnaíba sugere uma conexão entre esta bacia e a Bolívia, a qual teria ocorrido através da Subbacia Alto Garças (Bacia do Paraná), conforme sugere a descoberta desse táxon nesta localidade. A conexão da Bolívia com a Bacia do Parnaíba não teria ocorrido antes do Eifeliano, como indicam dados palinológicos (Lange, 1967);

- Metacryphaeus tuberculatus apresenta uma distribuição mais ampla do que se supunha. A espécie habitava áreas da Bolívia, Peru, e na Subbacia Apucarana no Eodevoniano. Posteriormente, no Mesodevoniano, M. tuberculatus migrou para a Subbacia Alto Garças e Bacia do Parnaíba.

- A maior parte dos trilobitas Calmoniidae revisados no Devoniano da Bacia do Amazonas pertence ao gênero Malvinella. Há dúvidas se o gênero Pennaia também estaria representado na localidade;

- A presença de Malvinella na Bacia do Amazonas reforça conclusões em trabalhos prévios (Lieberman et al., 1991; Lieberman, 1993) de que esta bacia apresentaria forte conexão com a Bolívia. A ausência do gênero nas demais bacias brasileiras sugere que a conexão a sudoeste da Bacia do Amazonas tenha sido a rota de migração dos Calmoniidae bolivianos, durante eventos transgressivos no Mesodevoniano;

- A distinção entre as espécies de Homalonotidae Burmeisteria notica e Burmeisteria herscheli são aparentemente mais sutis do que se imaginava. A presença de ornamentação externa em $B$. herscheli é a característica mais marcante para 
distinguir as espécies. Contudo, tal feição nem sempre está presente, visto que fatores tafonômicos podem obliterar a ornamentação (Simões et al., 2009).

- Na localidade do Morro do Ranulfo, em João Costa, sudeste do estado do Piauí, ocorrem as espécies Burmeisteria notica e Metacryphaeus cf. australis (depois reconhecido como $M$. cf. meloi). A nova ocorrência destes trilobitas amplia a distribuição geográfica destas espécies no flanco leste da Bacia do Parnaíba, cujos afloramentos típicos encontram-se a do estado, na região dos municípios de Picos e Pimenteiras. 


\section{REFERÊNCIAS BIBLIOGRÁFICAS}

Angelim, L.A.A.; Vasconcelos, A.M. Gomes, J.R.C.; Wanderley, A.A.; Forgiarini, L.L.; Medeiros, M. de F. 2004. Folha SB.24 - Jaguaribe. In: Schobbenhaus, C.; Gonçalves, J.H.; Santos, J.O.S.; Abram, M.B.; Leão Neto, R.; Matos, G.M.M.; VidottI, R.M.; Ramos, M.A.B. \& Jesus, J.D.A. de. (eds.). Carta Geológica do Brasil ao Milionésimo, Sistemas de Informações Geográficas-SIG. Programa Geológico do Brasil, CPRM, Brasília. Disponível em: <http://geobank.sa.cprm.gov.br/>.

Almeida, F.F.M.; Hasui, Y.; Brito Neves, B.B. \& FUCK, R.A. 1977. Províncias estruturais brasileiras. In: SIMPÓSIO DE GEOLOGIA DO NORDESTE, 8, Campina Grande, 1977. Atas. P. 363-391.

Bosetti, E. P., Grahn, Y., Mendlowicz Mauller, P., Horodyski, R., Zabini, C., Breuer, P. 2011. An earliest Givetian "Lilliput Effect" in the Paraná Basin, and the collapse of the Malvinokaffric shelly fauna. Paläontol. Z. , 85: 49-65.

Boucot, A.J. (1988). Devonian biogeography: na update. In: N.J. McMillan, A.F. Embry, O.J. Glass (Eds.). Devonian of the world (v.1, 211-227). Calgary: Canadian Society of Petroleum Geologists.

Boucot, A.J., Johnson, J.G., Talent, J.A. 1969. Early Devonian brachiopod zoogeography. Geological Society of America Special Paper 119.

Caputo,M.V.; IANNUZZI,R. \& Fonseca,V.M.M. 2005. Bacias sedimentares brasileiras: Bacia do Parnaíba. Phoenix, 81: 1-6.

Caputo, M.V. \& Ponciano, L.C.M.O. 2010. Pavimento Estriado de Calembre, Brejo do Piauí - Registro de geleiras continentais há 360 milhões de anos no Nordeste do Brasil. In: WINGE, M.; SCHOBBENHAUS, C.; SOUZA, C.R.G.; FERNANDES, A.C.S.; BERBERT-BORN, M.; SALLUN FILHO, W. \& QUEIROZ, E.T.; (eds.) Sítios Geológicos e Paleontológicos do Brasil. Disponível em: <35TTP://www.unb.br/ig/sigep/sitio052/sitio052.pdf>.

Carvalho, I.S.; Vicalvi, M.A. \& Carvalho, M.G.P. 2004. Artrópodes. In: Carvalho, I.S. (ed.). Paleontologia. Rio de Janeiro: Interciência, 2004, 2a ed. v.1, p. 579-607. 
Carvalho, M.G.P. 1995. Trilobitas devonianos da Bacia do Parnaíba (formações Pimenteira, Cabeças e Longá). Programa de Pós-graduação em Geologia, Universidade Federal do Rio de Janeiro, Tese de Doutoramento, 132p.

Carvalho, M.G.P. 1999. Remarks on the calmoniid trilobites from the Middle Devoniano of the Parnaíba Basin, NE Brazil. In: CONGRESSO BRASILEIRO DE PALEONTOLOGIA, 16, Crato, 1999. Boletim de Resumos. p. 34.

Carvalho, M.G.P. 2006. Devonian trilobites from the Falkland Islands. Palaeontology, 49(1): 21-34.

Carvalho, M.G.P.; Edgecombe, G.D. \& Lieberman, B.S. 1997. Devonian calmoniid trilobites from the Parnaíba Basin, Piauí State, Brazil. American Museum Novitates, 3192: 1-11.

Carvalho, M.G.P.; Edgecombe, G.D. \& Smith, L. 2003. New calmoniid trilobites (Phacopina: Acastoidea) from the Devonian of Bolivia. American Museum Novitates, 3407: 1-17.

Carvalho, M.G.P. \& Fonseca, V.M.M. 2007. The trilobite "Dalmanites" maecurua Clarke, 1890 (Middle Devonian, Amazon Basin, Brazil) and the new genus Amazonaspis (Synphoriidae). American Museum Novitates, 3591: 1-16.

Carvalho, M.G.P., Ponciano, L.C.M.O (2015). Devonian trilobites from the Amazonas Basin: systematic diversity, taphonomy and biogeographic significance. Boletim do Museu Paraense Emílio Goeldi. Ciências Naturais, 10 (1), 37-48.

Clarkson, E.N.K. 1998. Invertebrate Palaeontology and Evolution. Oxford, Blackwell Science, $4^{\text {th }}$ edition. $452 \mathrm{p}$.

Clarke, J.M. 1890. As trilobitas do Grez de Ererê e Maecuru, Estado do Pará, Brazil. Archivos do Museu Nacional, 9: 1-58.

Clarke, J.M. 1913. Fósseis devonianos do Paraná. Monografia do Serviço Geológico e Mineralógico do Brasil, 1:1-353.

Castro, J.S. 1968. Trilobitas da Formação Pimenteiras, Devoniano do estado do Piauí. Anais da Academia Brasileira de Ciências, 40(4): 481-489.

Cooper, M.R. 1982. A revision of the Devonian (Emsian-Eifelian)Trilobita from the Bokkeveld Group of South Africa. Annals of The South African Museum, 89:1-174. 
Cunha, P.R.C. 2000. Análise estratigráfica dos sedimentos eo/mesodevonianos da porção ocidental da Bacia do Amazonas sob a ótica da estratigrafia de sequências no interior cratônico. Programa de Pós-graduação em Geociências, Universidade Federal do Rio Grande do Sul, Dissertação de Mestrado, 263p.

Cunha, P.R.C.; Melo, J.H.G. \& Silva, O.B. 2007. Bacia do Amazonas. Boletim de Geociências da Petrobras, 15 (2): 227-251.

Della Fávera, J.C. 1990. Tempestitos da Bacia do Parnaíba. Um ensaio holístico. Programa de Pós-graduação em Geociências, Universidade Federal do Rio Grande do Sul, Tese de Doutoramento, 243p.

Derby, O.A. 1878. Contribuições para a geologia da região do baixo Amazonas. Archivos do Museu Nacional, 2: 77-104.

Duarte, A. 1936. Fósseis da sondagem de Therezina, Estado do Piauhy. Notas Preliminares e Estudos do Serviço Geológico e Mineralógico (2): 1-3.

Eldredge, N. \& Ormiston, A.R. 1979. Biogeography of Silurian and Devonian Trilobites of the Malvinokaffric Realm. In: Boucot, A.J. \& Gray,J.(eds.). Historical Biogeography, Plate Tectonics, and Changing Environment. Oregon State Univ. Press, Corvalis, p. 147-167.

Eldredge, N. \& Braniša, L. 1980. Calmoniid trilobites of the Lower Devonian Scaphiocoelia Zone of Bolivia, with remarks on related species. Bulletin of the American Museum of Natural History, 165 (2):181-289.

Fonseca, V.M.M. \& Melo, J.H.G. 1987. Ocorrência de Tropidoleptus carinatus (Conrad) (Brachiopoda, Orthida) na Formação Pimenteira, e sua Importância Paleobiogeográfica. In: CONGRESSO BRASILEIRO DE PALEONTOLOGIA, 10, 1987. Anais, Rio de Janeiro, PETROBRAS, p.505-537.

Fonseca, V.M.M. \& Ponciano, L.C.M.O. 2011. Braquiópodes do Devoniano Médio das bacias do Amazonas e Parnaíba. In: CARVALHO, I.S.; SRIVASTAVA, N.K.; STROHSCHOEN Jr., O.; LANA, C.C. (org.). Paleontologia: cenários de vida. Rio de Janeiro: Interciência, 2011, v. 4, p. 169-190.

Fonseca, V.M.M. 2015. Considerações paleobiogeográficas baseadas em braquiópodes devonianos (Delthyridoidea, Strophodontoidea e Chonetoidea) das bacias do 
Amazonas e Parnaíba. . Boletim do Museu Paraense Emílio Goeldi. Ciências Naturais, 10 (1), 91-101.

Ghilardi, R.P. \& Simões, M.G. 2007. History and Development of Trilobite Research in Brazil. In: Mikulic, D.G.; Landing, E. \& Kluessendorf, J. (eds.). Fabulous Fossils: 300 Years of Worldwide Research on Trilobites. New York State Museum Bulletin 507, Albany, p. 97-104.

Grahn, Y. 1992. Revision of Silurian and Devonian strata of Brazil. Palynology, 16:3561.

Grahn, Y. \& Melo, J.H.G. 2004. Integrated Middle Devonian chitinozoan and miospore zonation of the Amazonas Basin, northern Brazil. Revue de Micropaléontologie, 47:71-85.

Grahn, Y. 2005. Devonian chitinozoan biozones of Western Gondwana. Acta Geologica Polonica, 55:211-227.

Grahn,Y.; Young, C. \& Borghi, L. 2008. Middle Devonian chitinozoan biostratigraphy and sedimentology in the eastern outcrop belt of the Parnaíba Basin, northeastern Brazil. Revista Brasileira de Paleontologia, 11(3):137-146.

Góes, A.M.O. 1995. A Formação Poti (Carbonífero Inferior) da Bacia do Parnaíba. Programa de Pós-Graduação em Geologia Sedimentar, Universidade de São Paulo, São Paulo, Tese de Doutoramento, 171 p.

Harrington, H.J., Henningsmoen, G., Howell, B.F., Jaanusson, V., Lochman-Balk, C., Moore, R.C., Poulsen, C., Rasetti, F., Richter, E., Schmidt, H., Sdzuy, K., Struve, W., Størmer, L., Stubblefield, C.J., Tripp, R., Weller, J.M., Whittington, H.B. (1959). Trilobita. In: Moore, R. C. (Ed.). Treatise on Invertebrate Paleontology Part O Arthropoda (O38-O526). Lawrence: Geological Society of America and University of Kansas Press.

Hartt, C. F. \& Rathbun, R., 1875, Devonian trilobites and mollusks of Ererê, Province of Pará, Brazil. Annals Lyceum Natural History, 11: 111-127.

Isaacson, P.E. \& Díaz Martínez, E. 1995. Evidence for a middle-late Paleozoic foreland basin and significant paleolatitudinal shift, central Andes. In.: Tankard, A.J., 
Suárez-Soruco, R \& Welsink, H.J. Petroleum basins of South America: AAPG Memoir 62: p. 231-249.

Katzer, F. 1898. A fauna devônica do Rio Maecuru e as suas relações com a fauna de outros terrenos devônicos do globo. Boletim do Museu Paraense Emílio Goeldi, vol. 2, p. 204-246.

Kegel, W. 1953. Contribuição para o estudo do Devoniano da Bacia do Parnaíba. Boletim da Divisão de Geologia e Mineralogia, 141: 1-48.

Lange, F. W. 1954. Paleontologia do Paraná, volume comemorativo do $100^{\circ}$ aniversário do Estado do Paraná, Curitiba, Brazil, p. 1-106.

Lange, F. W. \& Petri, S. 1967. The Devonian of the Paraná Basin. Boletim Paranaense de Geociências (21-22): 5-55.

Levi-Setti, R. 1995. Trilobites (second edition). Chicago and London, The University of Chicago Press, 352 p.

Lieberman, B. S. 1993. Systematics and biogeography of the 'Metacryphaeus Group' Calmoniidae (Trilobita, Devonian), with comments on adaptative radiations and the geological history of the Malvinokaffric Realm. Journal of Paleontology, 67: 549570.

Lieberman, B. S.; Edgecombe, G. D., and Eldredge, N., 1991, Systematics and biogeography of the "Malvinella group", Calmoniidae (Trilobita,Devonian): Journal of Paleontology, 65(5): 824-843.

Lima, E.A.M. \& Leite, J.F. 1978. Projeto estudo global dos recursos minerais da Bacia Sedimentar do Parnaiba: integração geológico-metalogenética. Relatório final da etapa III. Companhia de Pesquisa de Recursos Minerais, 212 p

Lucas, S.G. 2001. Taphotaxon. Lethaia, 34: 30.

Melo, J.H.G., 1985. A província Malvinocáfrica no Devoniano do Brasil: estado atual dos conhecimentos. Programa de Pós-graduação em Geologia, Universidade Federal do Rio de Janeiro, Dissertação de Mestrado, 890p.

Melo, J.H.G., 1988 - The Malvinokaffric realm in the Devonian of Brazil. In: McMILLAN, N.J.; EMBRY, A.F. \& GLASS, O.J. (eds.). Devonian of the world. Canadian Society of Petroleum Geologists, Proceedings of the $2^{\text {nd }}$ International 
Symposium on the Devonian System, Calgary, C.S.P.G., 1987 Memoir, 14 (1): 667-703.

Melo, J.H.G. \& Loboziak, S. 2003. Devonian - Early Carboniferous miospore biostratigraphy of the Amazon Basin, Northern Brazil. Review of Paleobotany and Palynology, 124 (3-4):131-202.

Mori, A.S. 2013. Revisão sistemática de Calmoniidae (Trilobita, Phacopida) da Formação Ponta Grossa, Devoniano, Bacia do Paraná, Brasil. Programa de Pósgraduação em Geoquímica e Geotectônica, Universidade de São Paulo, Dissertação de Mestrado, 67p.

Morzadec, P., Mergl, M., Villarroel, C., Janvier, P., Racheboeuf, P. (2015). Trilobites and inarticulate brachiopods from the Devonian Floresta Formation of Colombia: a review. Bulletin of Geosciences, 90 (2), 331-358.

Nascimento, S.; Lemos, V. B.; Scomazzon, A. K. \& Moutinho, L. P. 2006. Bioestratigrafia de Conodontes na Borda Sul da Bacia do Amazonas, Pará - Brasil.. In: Semana Acadêmica dos Alunos de Pós-Graduação em Geociências, UFRGS, 1, Porto Alegre, 2006, Resumos. P. 109-112.

Oliveira, A.I., Leonardos, O.H. (1978). Geologia do Brasil ( $3^{\text {rd }}$ Ed.). Mossoró, Escola Superior de Agricultura de Mossoró.

PALAEOS: Life through deep time. Disponível em: <http://palaeos.com/>. Acesso em 26 fev. 2016.

Playford, G. \& Dino, R. 2000. Palynostratigraphy of upper Paleozoic strata (Tapajós Group), Amazonas Basin, Brazil. Palaeontographica Abteilung B, 255:1-46.

Ponciano, L.C.M.O. \& Machado, D.M.C.2007. Macroinvertebrados da Formação Maecuru (Devoniano, Bacia do Amazonas, Brasil) - considerações tafonômicas. In: Carvalho, I.S.; Cassab, R.C.T.; Schwanke, C.; Carvalho, M.A.; Fernandes, A.C.S.; Rodrigues, M.A.C.; Carvalho, M.S.S.; Irai, M.; Oliveira, M.E.Q. (eds.). Paleontologia: cenários de vida. Rio de Janeiro: Interciência, 2007, v. 1, p. 131137.

Ponciano, L.C.M.O.; Fonseca, V.M.M.; Fernandes, A.C.S.; Machado, D.M.C. \& Souza, A.R. 2010. Afloramento Fossilífero de Oiti, Bacia do Parnaíba, PI: Registro de um 
mar devoniano no Nordeste do Brasil. In: Winge, M.; Schobbenhaus, C.; Souza, C.R.G.; Fernandes, A.C.S.; Berbert-Born, M.; Sallun filho, W. \& Queiroz, E.T. (eds.). Sítios Geológicos e Paleontológicos do Brasil. Disponível em: <http://www.unb.br/ig/sigep/sitio051/sitio051.pdf>.

Popp, M.T.B. 1985. Revisão dos trilobitas calmoniideos e comunidades faunísticas da Formação Ponta Grossa, Devoniano, no Estado do Paraná. Programa de Pósgraduação em Geociências, Universidade Federal do Rio Grande do Sul, Tese de Doutoramento, 112p.

Quadros, L.P. 1982. Distribuição bioestratigráfica dos Chitinozoa e Acritarchae na bacia do Parnaíba. Ciência-Técnica-Petróleo,Seção: Exploração de Petróleo, 12:1-76.

Rezende, N. G. A. M. 2002. A zona zeolítica da Formação Corda, Bacia do Parnaíba. Curso de Pós-graduação em Geologia e Geoquímica, Universidade Federal do Pará, Belém, Dissertação de Mestrado, 142 p.

Rossetti, D.F.; Góes, A.M.O. \& Arai, M. 2001. A passagem Aptiano-Albiano na Bacia do Grajaú, MA. In: Rossetti, D.F.; Góes, A.M.O. \& Truckenbrodt, W. (eds.). $O$ Cretáceo na Bacia de São Luís-Grajaú. Belém: Museu Paraense Emilio Goeldi, 2001. P.101-117.

Sandford, A.C. 2005. Homalonotid trilobites from the Silurian and Lower Devonian of south-eastern Australia and New Zealand (Arthropoda: Trilobita: Homalonotidae). Memoirs of Museum Victoria, 62 (1): 1-66.

Scholtz, G. \& Edgecombe, G.D. 2005. Heads, Hox and the phylogenetic position of trilobites. Crustac. Issues 16: 139-165.

Schobbenhaus, C. \& Brito Neves, B.B. 2003. A Geologia do Brasil no Contexto da Plataforma Sul-Americana. In: Bizzi, L.A.; Schobbenhaus, C.; Vidotti, R.M. \& Gonçalves, J.H. (eds.). Geologia, Tectônica e Recursos Minerais do Brasil. CPRM, Brasília, 2003. p. 6-54.

Silva, C.F. \& Fonseca, V.M.M. 2005. Hábitos de vida dos trilobitas das formações Maecuru e Ererê, Devoniano da Bacia do Amazonas, Brasil. Revista Brasileira de Paleontologia, 8 (1): 73-82. 
Simões, M.G.; Leme, J.M. \& Soares, S.P. 2009. Systematics, taphonomy and paleoecology of Homalonotid trilobites (Phacopida) from the Ponta Grossa Formation (Devonian), Paraná Basin, Brazil. Revista Brasileira de Paleontologia, 12 (1): $27-42$.

Soares, S.P. 2007. Sistemática, Tafonomia e Paleoecologia de Trilobita Phacopida (Homalonotidae e Calmoniidae), Formação Ponta Grossa (Devoniano), Sub-Bacia Apucarana, Estado do Paraná, Brasil. Programa de Pós-Graduação em Geologia Sedimentar, Universidade de São Paulo, São Paulo, Dissertação de Mestrado, 140 p.

Soares, S.P.; Simões, M.G. \& Leme, J.M. 2008a. Metacryphaeus rotundatus, um Novo Elemento da Fauna de Trilobites Calmoniidae (Phacopida), da Formação Ponta Grossa (Devoniano), Bacia do Paraná, Brasil. Revista do Instituto de Geociências USP, 8 (1): 15-24.

Soares, S.P.; Simões, M.G. \& Leme, J.M. 2008b. O papel da fossilização e do intemperismo na sistemática dos trilobites Phacopida (Calmoniidae e Homalonotidae), Formação Ponta Grossa, (Devoniano), Bacia do Paraná, Brasil. Revista Brasileira de Paleontologia, 11 (2): 117-128.

Vasconcelos, A.M.; Kosin, M.; Souza, J.D. de; Valente, C.R.; Neves, J.P.; Heineck, C.A.; Lacerda Filho, J.V.; Teixeira, L.R.; Borges, V.P.; Bento, R.V.; Guimarães, J.T.; Oliveira, I.W.B.; Gomes, I.P.; Malouf, R.F.; Carvalho, L.M. de \& Abreu Filho, W. 2004. Folha SC.23 - Rio São Francisco. In: Schobbenhaus, C.; Gonçalves, J.H.; Santos, J.O.S.; Abram, M.B.; Leão Neto, R.; Matos, G.M.M.; Vidotti, R.M.; Ramos, M.A.B. \& Jesus, J.D.A. de. (eds.). Carta Geológica do Brasil ao Milionésimo, Sistemas de Informações Geográficas-SIG. Programa Geológico do Brasil, CPRM, Brasília. Disponível em: $<$ http://geobank.sa.cprm.gov.br/>.

Vaz, P.T.; Rezende, N.G.A.M.; Wanderley Filho, J.R. \& Travassos, W.A.S. 2007. Bacia do Parnaíba. Boletim de Geociências da Petrobras, 15 (2): 253-263.

Whittingon, H.B.; Chatterton, B.D.E.; Speyer, S.E.; Fortey, R.A.; Owens, R.M.; Chang, W.T.; Dean, W.T.; Jell, P.A; Lawrie, J.; Palmer, A.R.; Repina, L.N.; Rushton, A.W.A.; Shergold, J.H.; Clarkson, E.N.K.; WilmoT, N.V. \& Kelly, S.R.A. 1997. 
Treatise on Invertebrate Paleontology, Pt. O, Arthropoda 1, Trilobita (revised edition). Lawrence, Geological Society of America and the University of Kansas Press, $530 \mathrm{p}$. 


\section{ANEXO I}

Relação do material estudado, depositado nas coleções nacionais e internacionais. 
Relação do material em estudo, depositado nas coleções nacionais e internacionais.

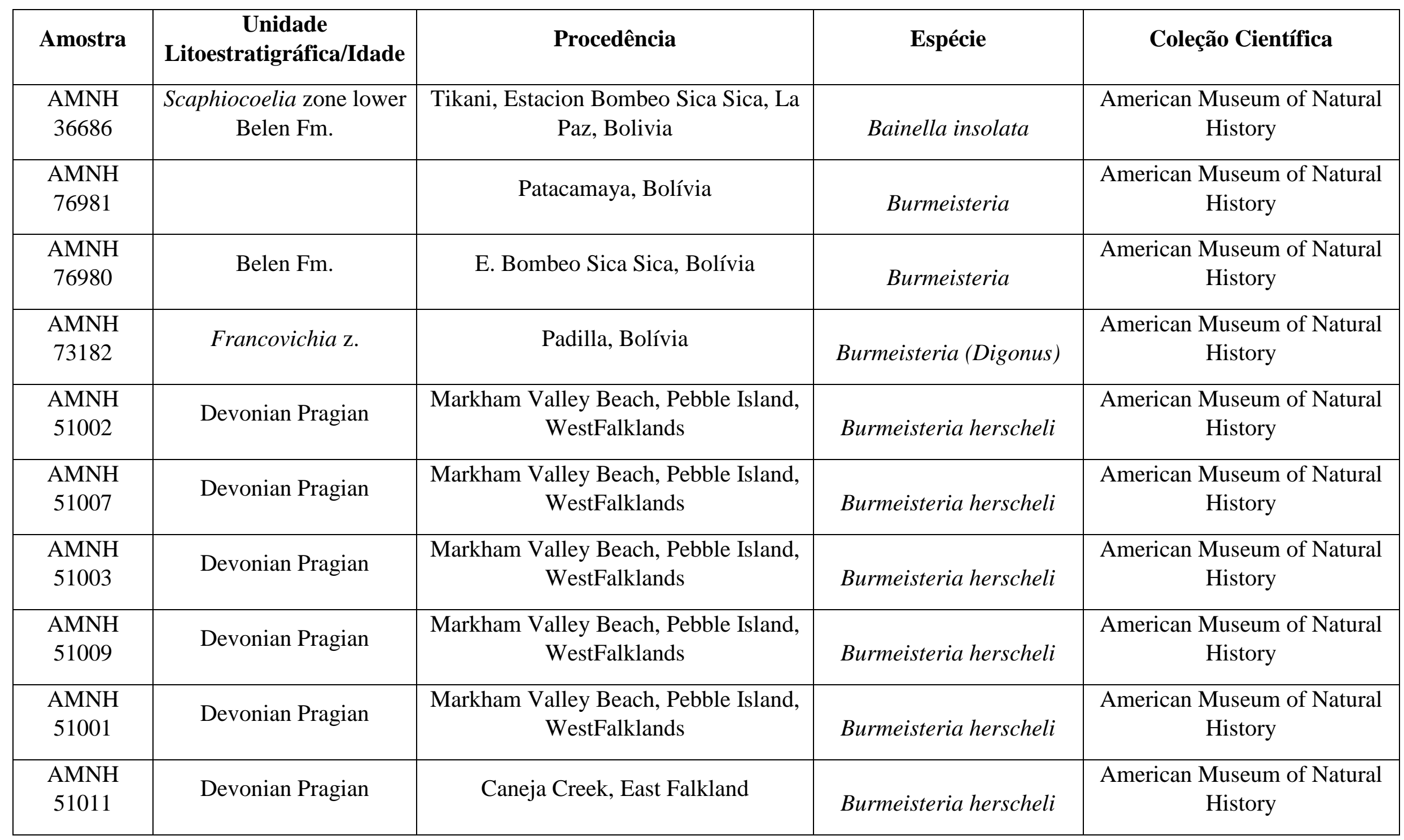




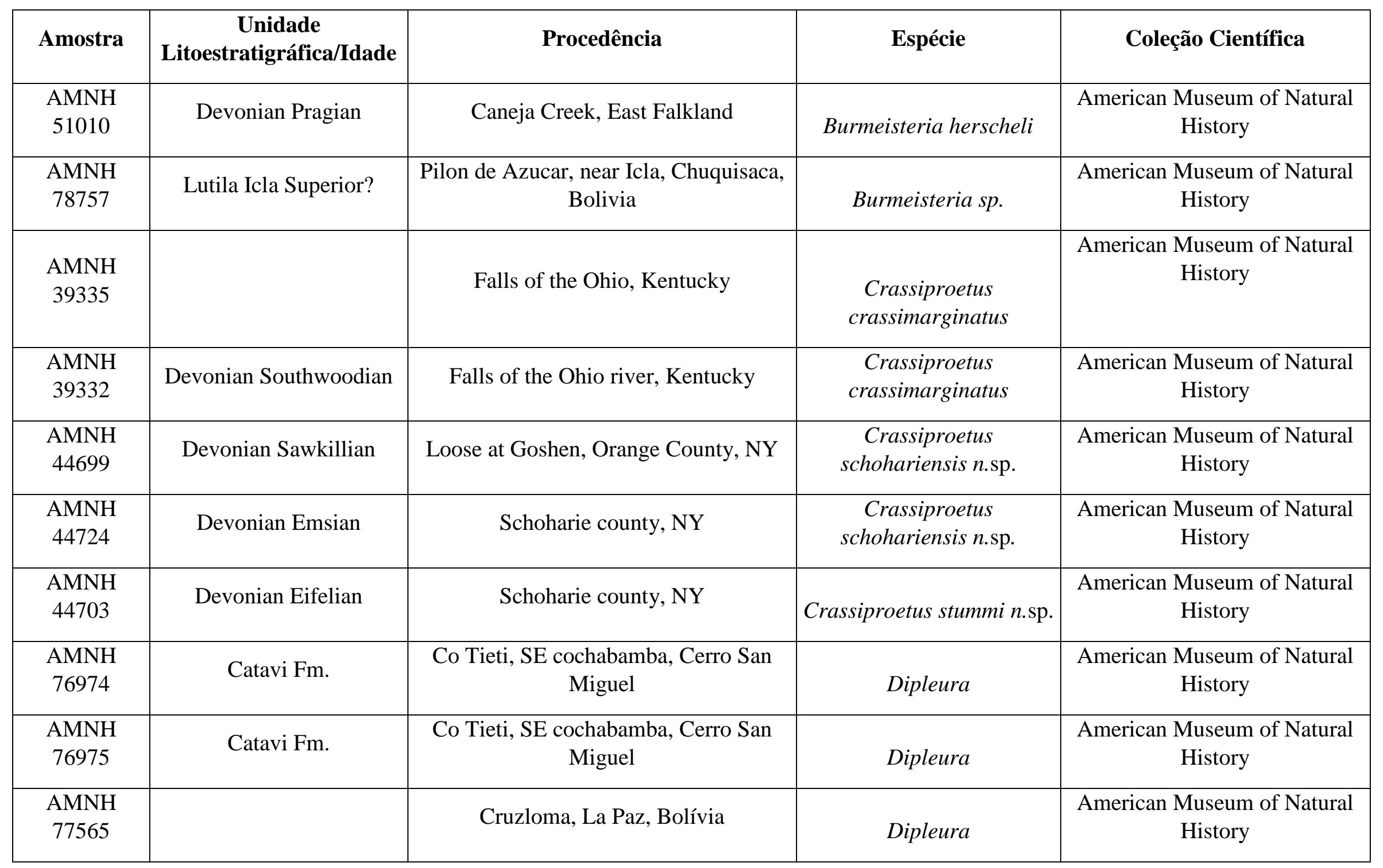




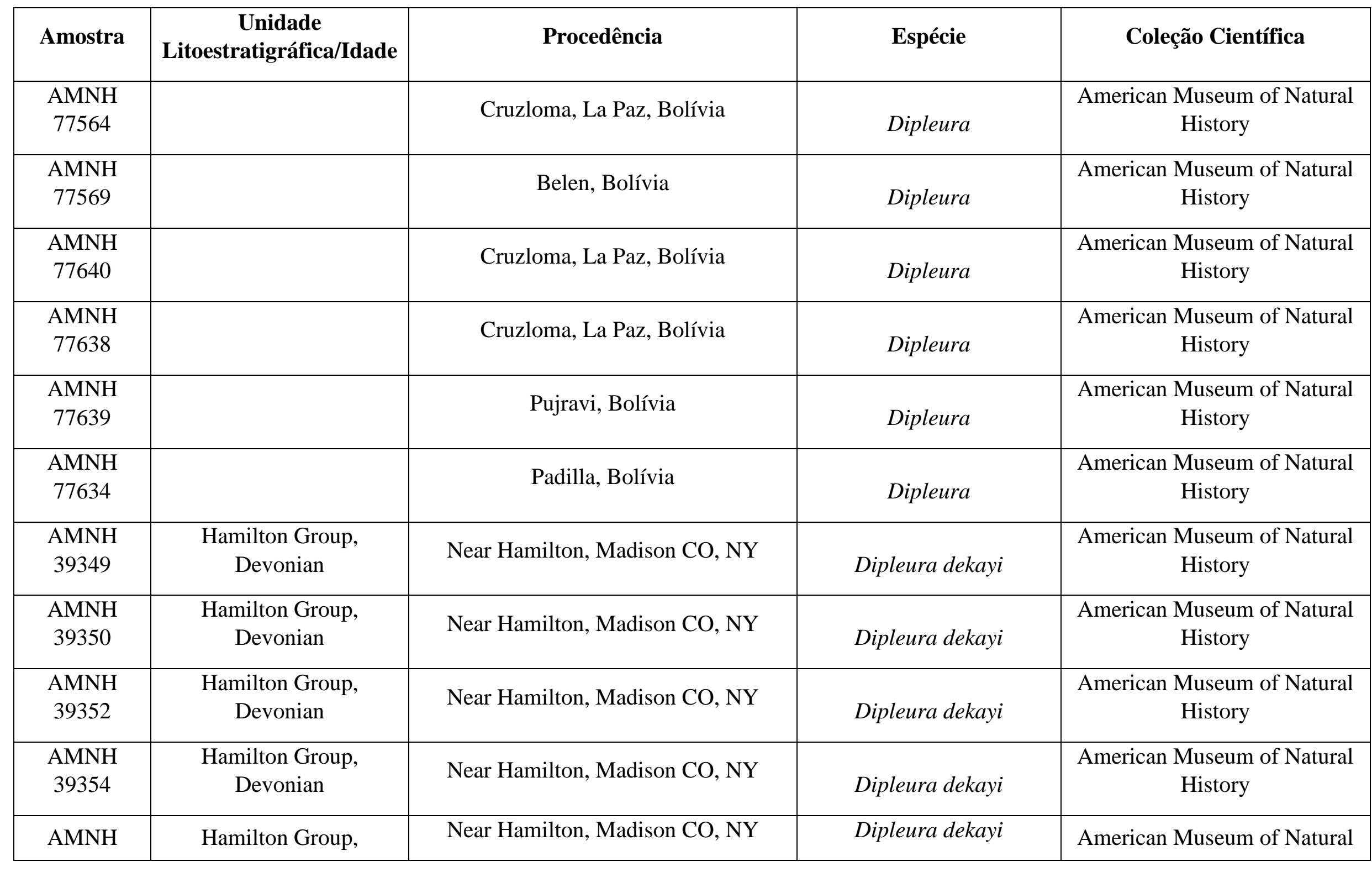




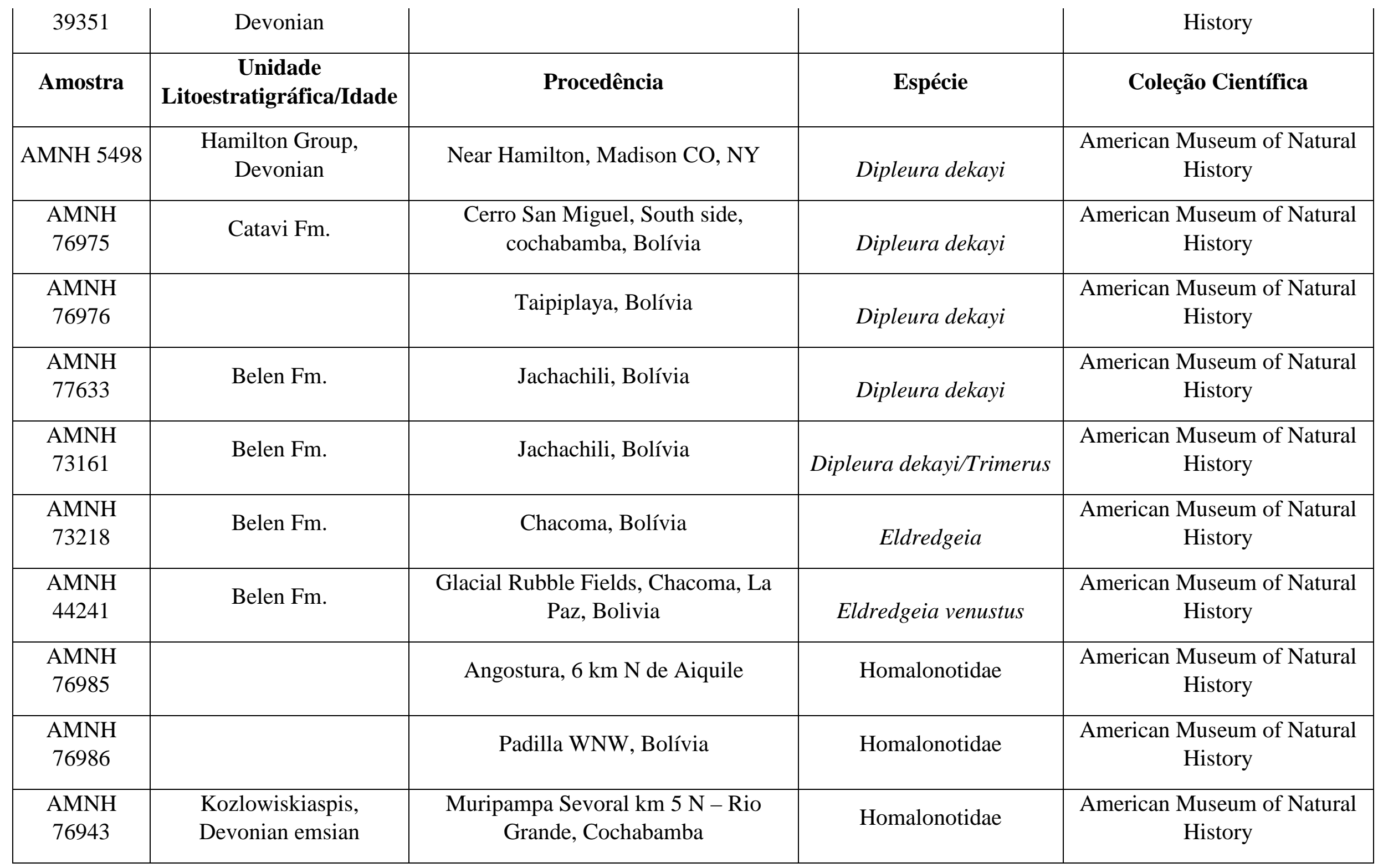




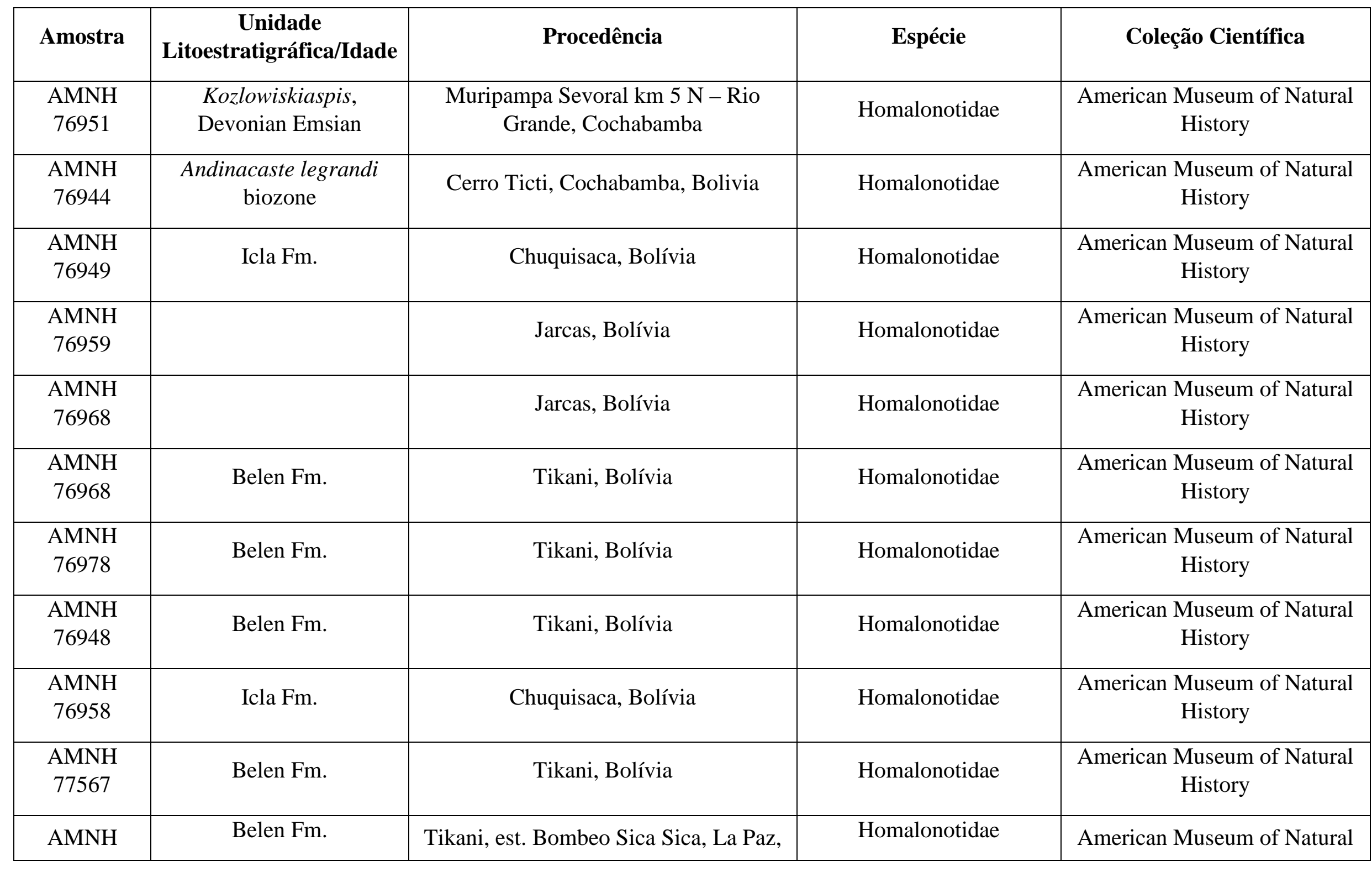




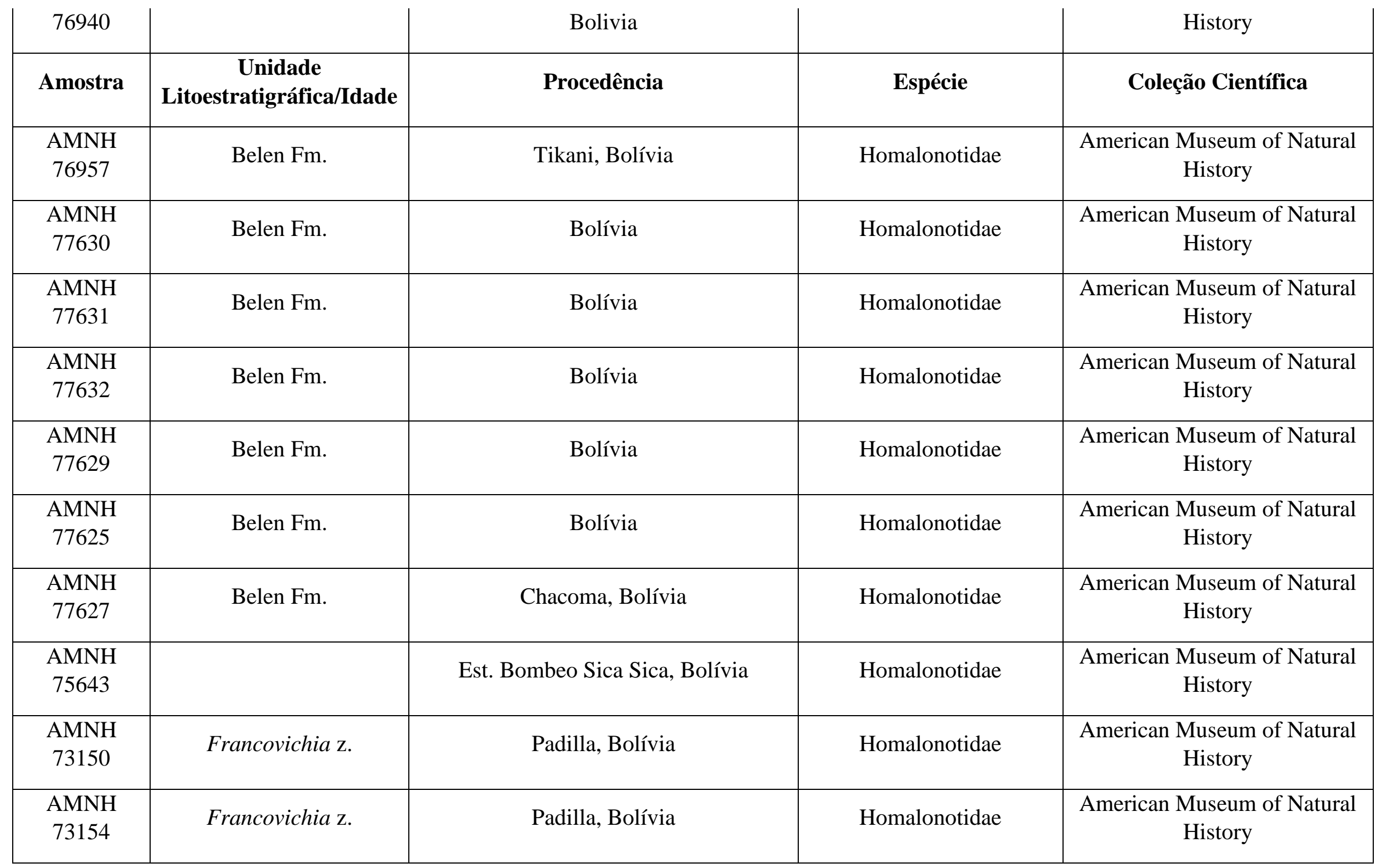




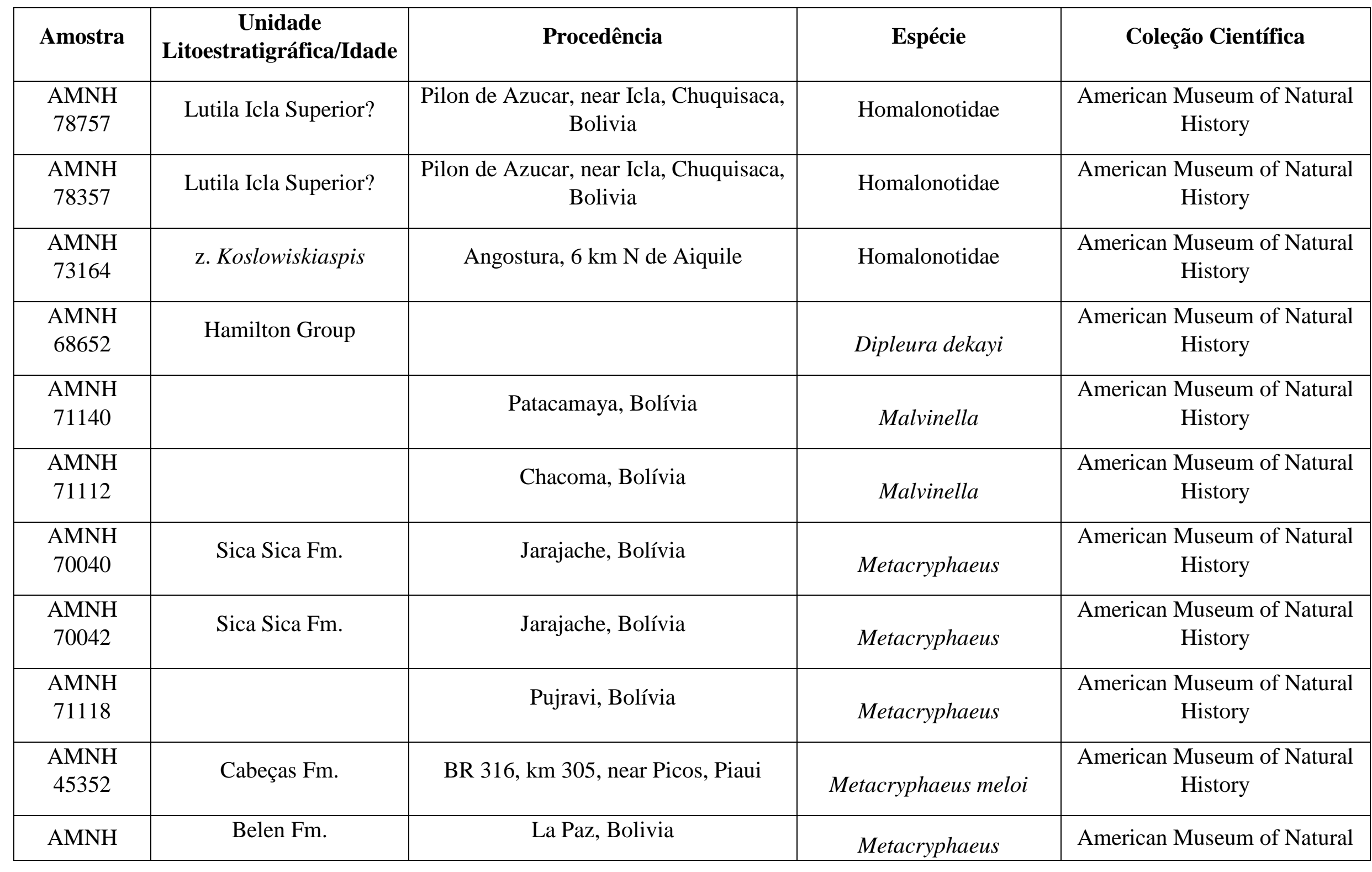




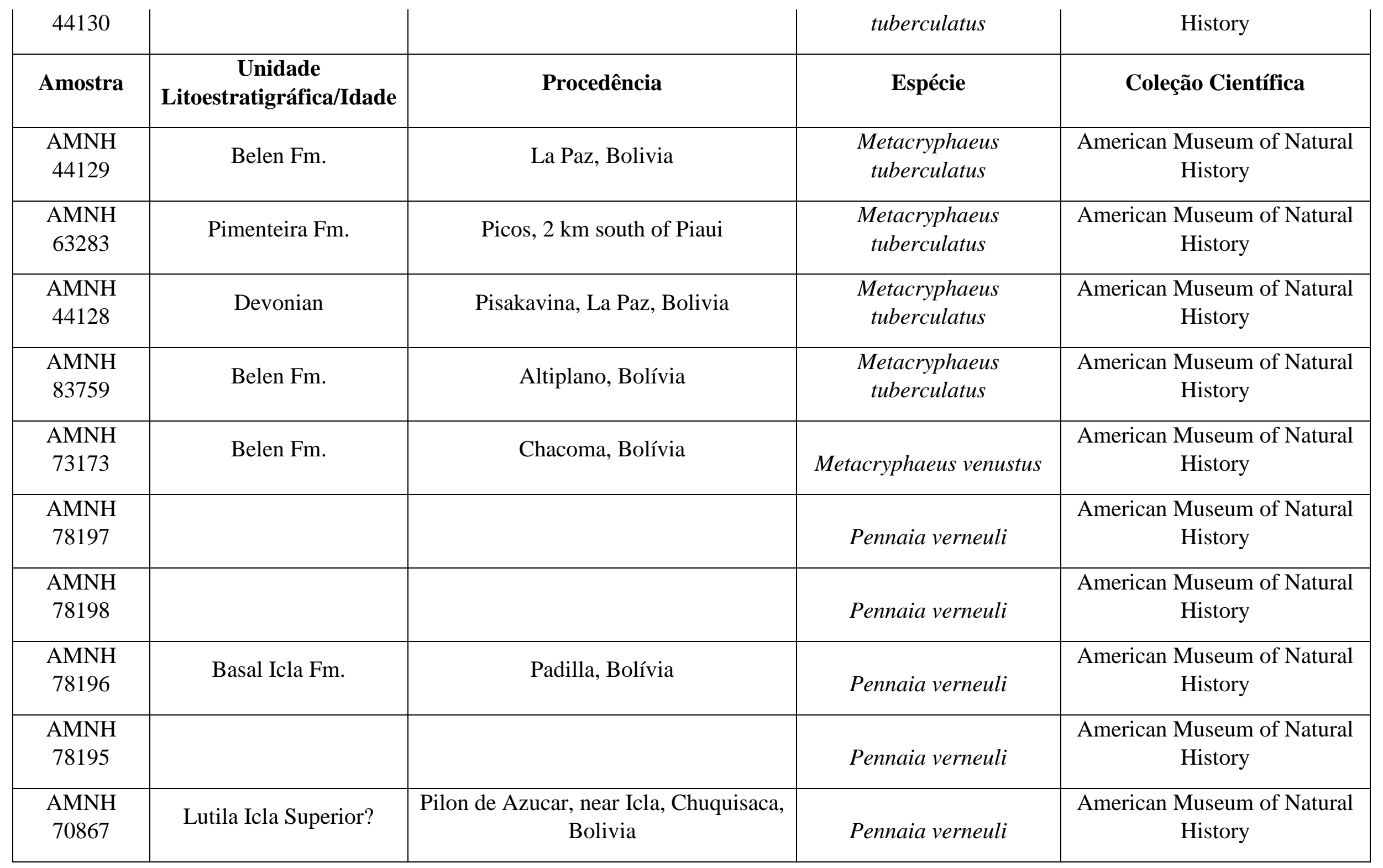




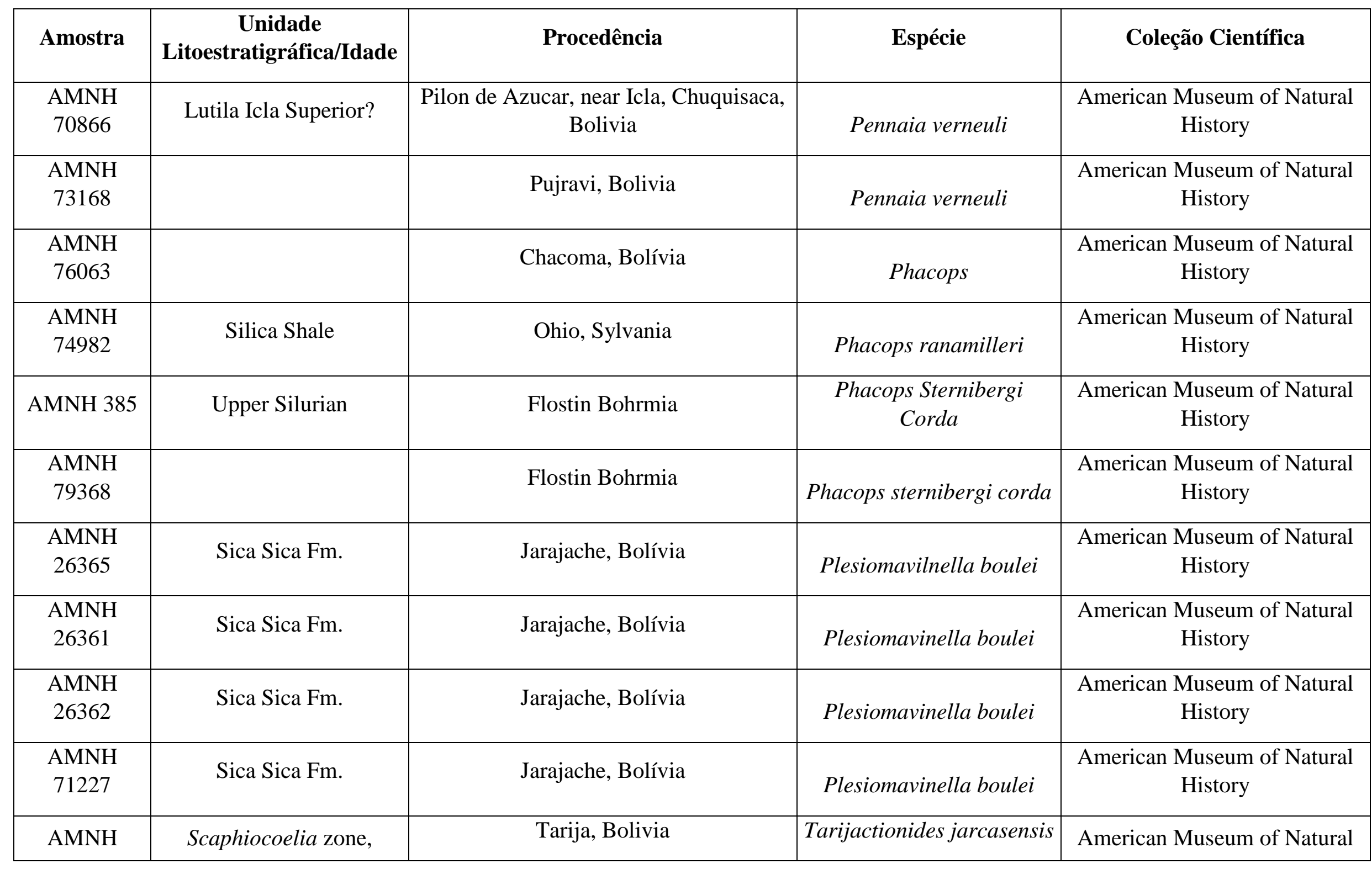




\begin{tabular}{|c|c|c|c|c|}
\hline 36749 & Gamoneda Fm. & & & History \\
\hline Amostra & $\begin{array}{c}\text { Unidade } \\
\text { Litoestratigráfica/Idade }\end{array}$ & Procedência & Espécie & Coleção Científica \\
\hline $\begin{array}{l}\text { AMNH } \\
76974\end{array}$ & & Tieti, Cochabamba, Bolívia & Trimerus & $\begin{array}{c}\text { American Museum of Natural } \\
\text { History }\end{array}$ \\
\hline $\begin{array}{l}\text { AMNH } \\
73170\end{array}$ & & Padilla, Bolívia & Trimerus & $\begin{array}{c}\text { American Museum of Natural } \\
\text { History }\end{array}$ \\
\hline $\begin{array}{l}\text { AMNH } \\
71639\end{array}$ & & $\begin{array}{c}\text { Eagle Furnace, Curtin, Centre Co, } \\
\text { Penna }\end{array}$ & Trimerus & $\begin{array}{c}\text { American Museum of Natural } \\
\text { History }\end{array}$ \\
\hline $\begin{array}{l}\text { AMNH } \\
31068\end{array}$ & Niagara Group Silurian & Niagara Shale, Lockport, NY & $\begin{array}{c}\text { Trimerus } \\
\text { delphinocephalus }\end{array}$ & $\begin{array}{c}\text { American Museum of Natural } \\
\text { History }\end{array}$ \\
\hline $\begin{array}{l}\text { AMNH } \\
31067\end{array}$ & Niagara Group Silurian & Niagara Shale, Lockport, NY & $\begin{array}{c}\text { Trimerus } \\
\text { delphinocephalus }\end{array}$ & $\begin{array}{c}\text { American Museum of Natural } \\
\text { History }\end{array}$ \\
\hline $\begin{array}{l}\text { AMNH } \\
40748\end{array}$ & Niagara Group Silurian & Indiana, Waldron & $\begin{array}{c}\text { Trimerus } \\
\text { delphinocephalus }\end{array}$ & $\begin{array}{c}\text { American Museum of Natural } \\
\text { History }\end{array}$ \\
\hline $\begin{array}{l}\text { AMNH } \\
40749\end{array}$ & Niagara Group Silurian & Indiana, Waldron & $\begin{array}{c}\text { Trimerus } \\
\text { delphinocephalus }\end{array}$ & $\begin{array}{c}\text { American Museum of Natural } \\
\text { History }\end{array}$ \\
\hline $\begin{array}{l}\text { AMNH } \\
40747\end{array}$ & Niagara Group Silurian & Indiana, Waldron & $\begin{array}{c}\text { Trimerus } \\
\text { delphinocephalus }\end{array}$ & $\begin{array}{c}\text { American Museum of Natural } \\
\text { History }\end{array}$ \\
\hline $\begin{array}{l}\text { AMNH } \\
42323\end{array}$ & Niagara Group Silurian & Niagara Shale, Lockport, NY & $\begin{array}{c}\text { Trimerus } \\
\text { delphinocephalus }\end{array}$ & $\begin{array}{c}\text { American Museum of Natural } \\
\text { History }\end{array}$ \\
\hline $\begin{array}{l}\text { AMNH } \\
31069\end{array}$ & Niagara Group Silurian & Niagara Shale, Lockport, NY & $\begin{array}{c}\text { Trimerus } \\
\text { delphinocephalus }\end{array}$ & $\begin{array}{c}\text { American Museum of Natural } \\
\text { History }\end{array}$ \\
\hline
\end{tabular}




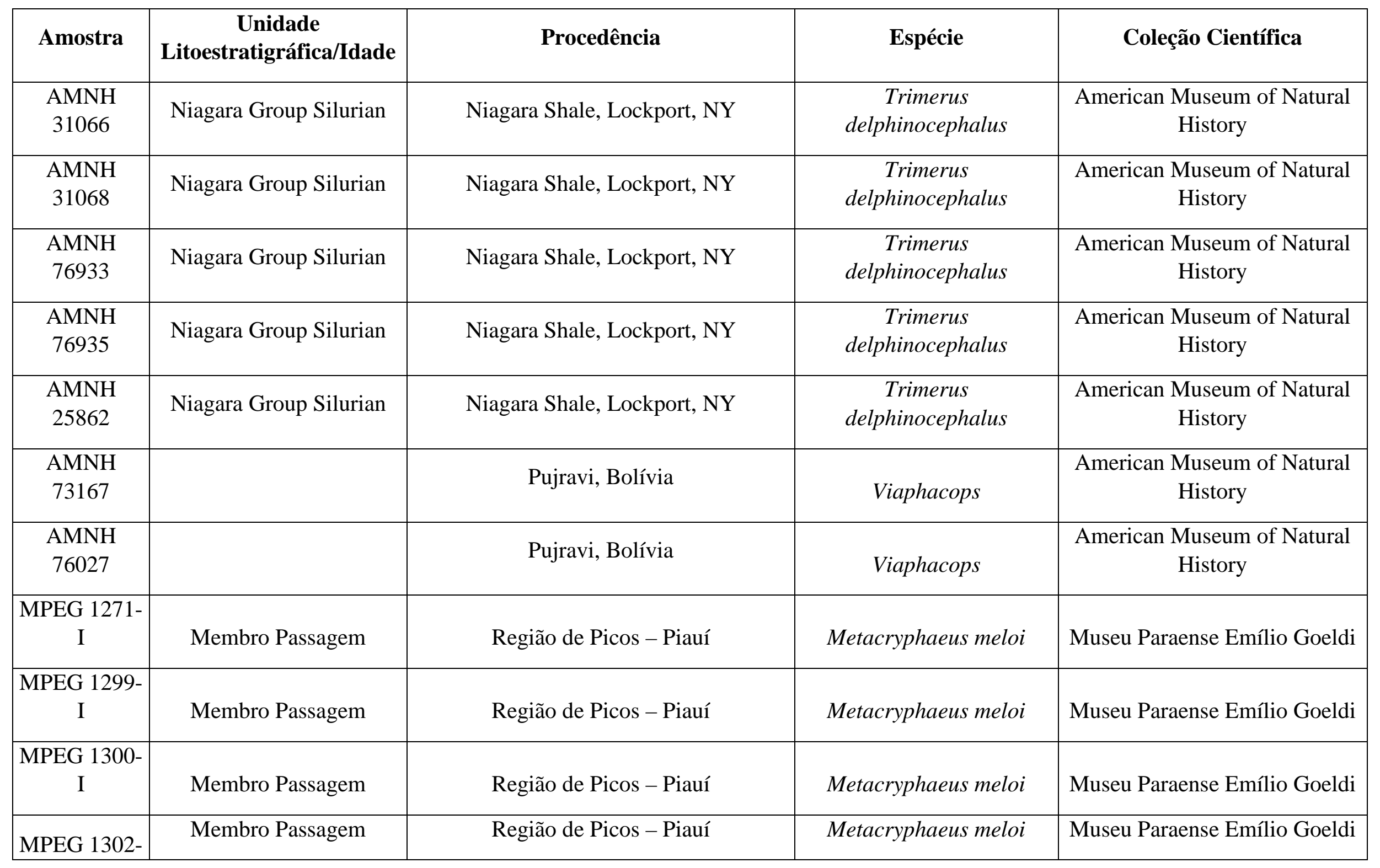




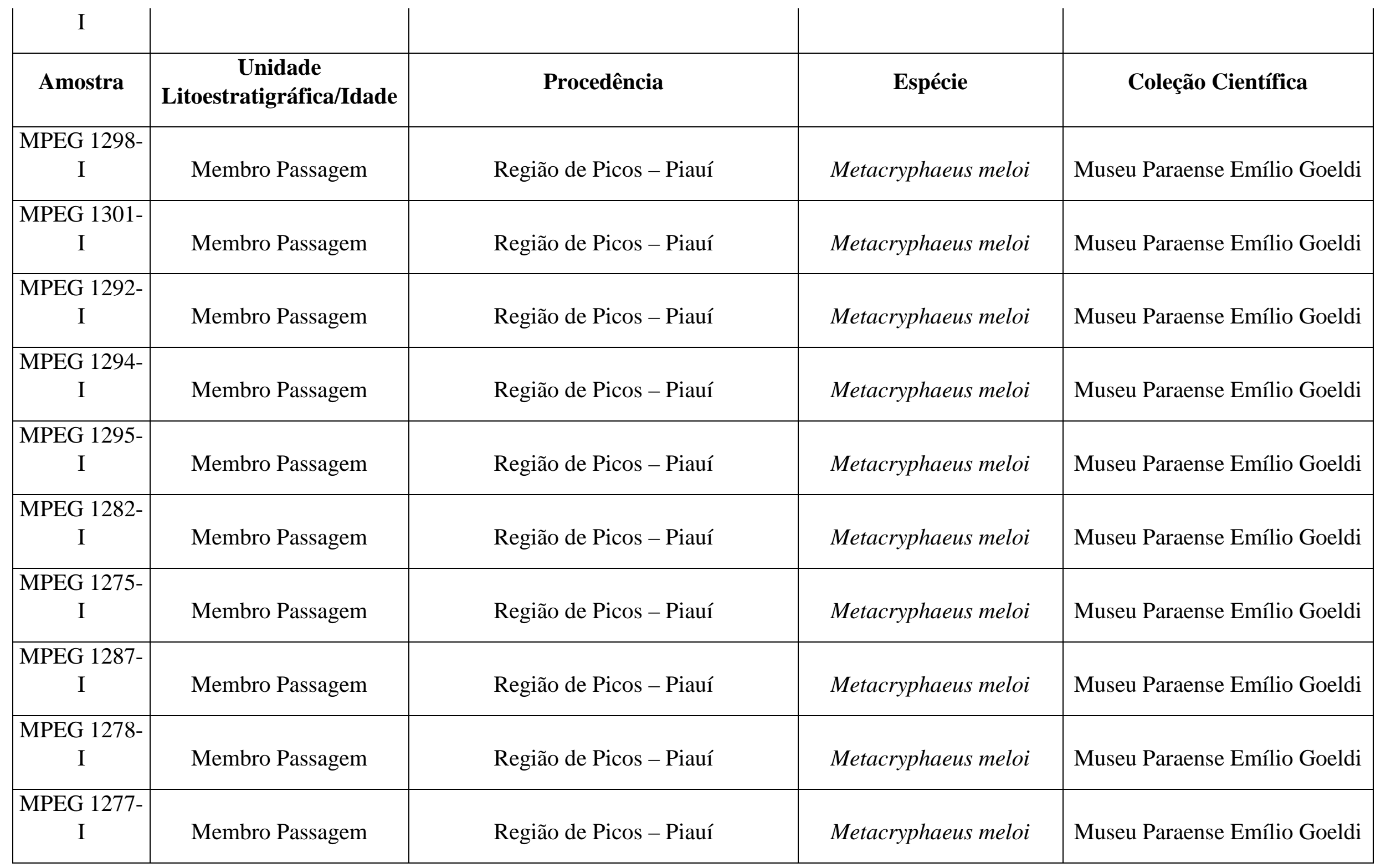




\begin{tabular}{|c|c|c|c|c|}
\hline Amostra & $\begin{array}{c}\text { Unidade } \\
\text { Litoestratigráfica/Idade }\end{array}$ & Procedência & Espécie & Coleção Científica \\
\hline \begin{tabular}{|c|} 
MPEG 1279- \\
I
\end{tabular} & Membro Passagem & Região de Picos - Piauí & Metacryphaeus meloi & Museu Paraense Emílio Goeldi \\
\hline $\begin{array}{l}\text { MPEG 1285- } \\
\text { I }\end{array}$ & Membro Passagem & Região de Picos - Piauí & Metacryphaeus meloi & Museu Paraense Emílio Goeldi \\
\hline $\begin{array}{l}\text { MPEG 1280- } \\
\text { I }\end{array}$ & Membro Passagem & Região de Picos - Piauí & Metacryphaeus meloi & Museu Paraense Emílio Goeldi \\
\hline \begin{tabular}{|} 
MPEG 1296- \\
I
\end{tabular} & Membro Passagem & Região de Picos - Piauí & Metacryphaeus meloi & Museu Paraense Emílio Goeldi \\
\hline \begin{tabular}{|c|} 
MPEG 1297- \\
I
\end{tabular} & Membro Passagem & Região de Picos - Piauí & Metacryphaeus meloi & Museu Paraense Emílio Goeldi \\
\hline \begin{tabular}{|c|} 
MPEG 1290- \\
I
\end{tabular} & Membro Passagem & Região de Picos - Piauí & Metacryphaeus meloi & Museu Paraense Emílio Goeldi \\
\hline $\begin{array}{l}\text { MPEG 1286- } \\
\text { I }\end{array}$ & Membro Passagem & Região de Picos - Piauí & Metacryphaeus meloi & Museu Paraense Emílio Goeldi \\
\hline $\begin{array}{l}\text { MPEG 1270- } \\
\text { I }\end{array}$ & Membro Passagem & Região de Picos - Piauí & Metacryphaeus meloi & Museu Paraense Emílio Goeldi \\
\hline MPEG 1281- & Membro Passagem & Região de Picos - Piauí & Metacryphaeus meloi & Museu Paraense Emílio Goeldi \\
\hline
\end{tabular}




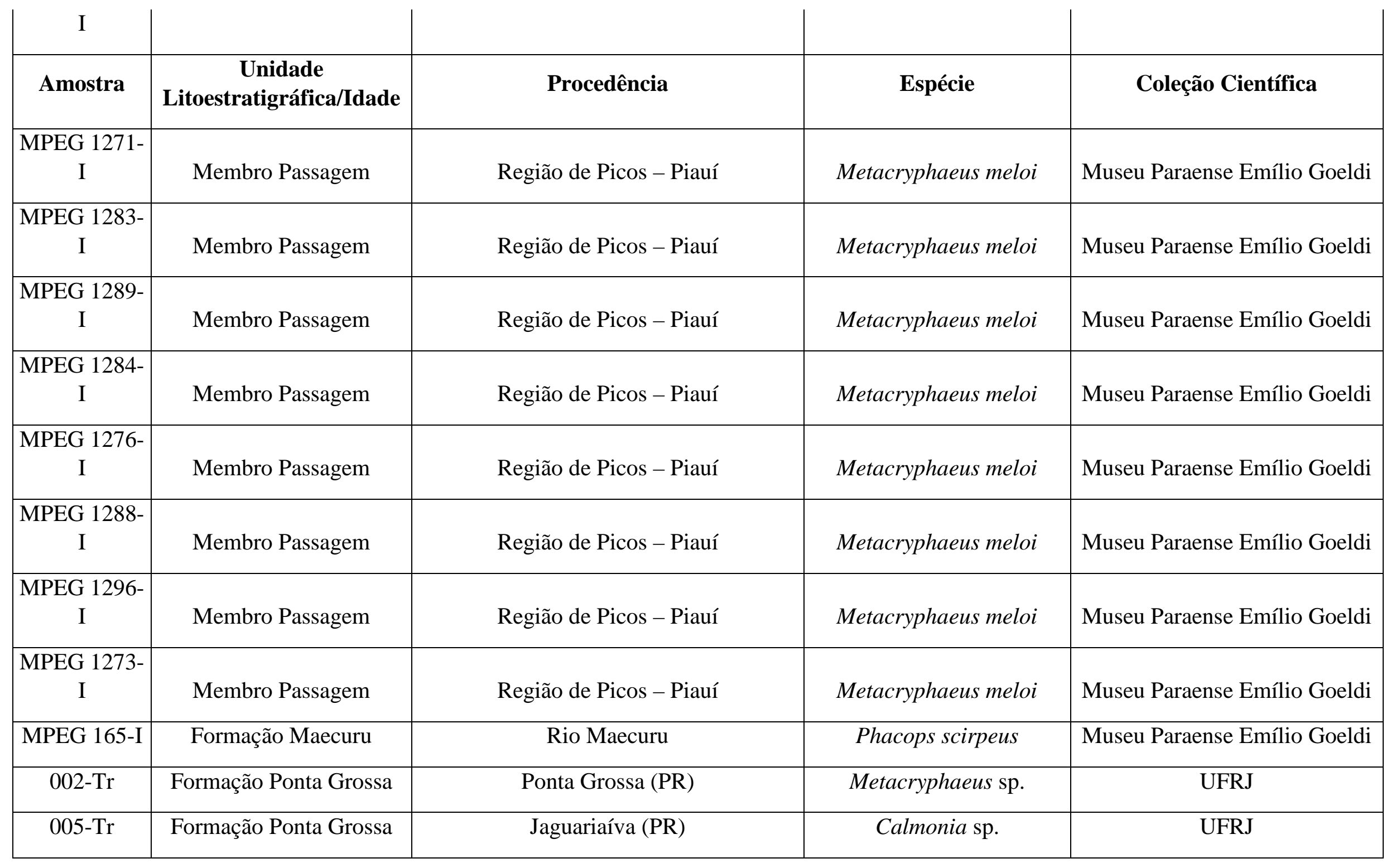




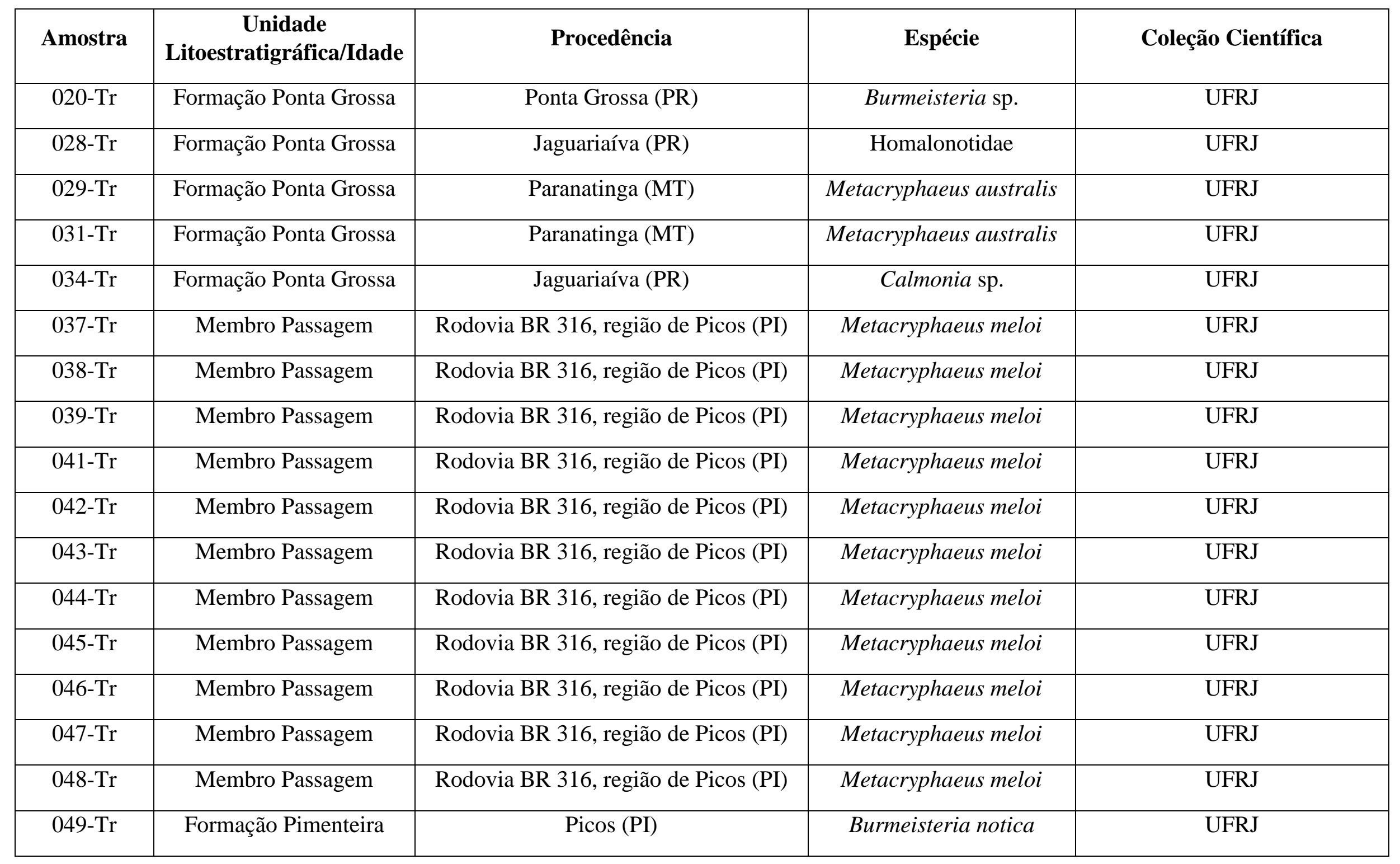




\begin{tabular}{|c|c|c|c|c|}
\hline Amostra & $\begin{array}{c}\text { Unidade } \\
\text { Litoestratigráfica/Idade }\end{array}$ & Procedência & Espécie & Coleção Científica \\
\hline $055-\operatorname{Tr}$ & Membro Passagem & Rodovia BR 316, região de Picos (PI) & Metacryphaeus meloi & UFRJ \\
\hline $057-\operatorname{Tr}$ & Membro Passagem & Rodovia BR 316, região de Picos (PI) & Metacryphaeus meloi & UFRJ \\
\hline $058-\operatorname{Tr}$ & Membro Passagem & Rodovia BR 316, região de Picos (PI) & Metacryphaeus meloi & UFRJ \\
\hline $060-\operatorname{Tr}$ & Formação Ponta Grossa & Ponta Grossa (PR) & Burmeisteria notica & UFRJ \\
\hline $068-\operatorname{Tr}$ & Formação Ponta Grossa & Ponta Grossa (PR) & Burmeisteria sp. & UFRJ \\
\hline $072-\operatorname{Tr}$ & Formação Ponta Grossa & Jaguariaíva (PR) & Metacryphaeus sp. & UFRJ \\
\hline $088-\operatorname{Tr}$ & Formação Ponta Grossa & Ponta Grossa (PR) & Calmonia sp. & UFRJ \\
\hline $089-\operatorname{Tr}$ & Formação Ponta Grossa & Ponta Grossa (PR) & Calmoniidae & UFRJ \\
\hline 093-Tr & & La Huachaca, Bolívia & Metacryphaeus sp. & UFRJ \\
\hline $102-\operatorname{Tr}$ & Formação Maecuru & Margem do Rio Maecuru & "Phacops" scirpeus & UFRJ \\
\hline $105-\mathrm{Tr}$ & Formação Maecuru & Margem do Rio Maecuru & "Phacops" macropyge & UFRJ \\
\hline 106-Tr & Formação Maecuru & Margem do Rio Maecuru & "Palpebrops" goeldi & UFRJ \\
\hline
\end{tabular}




\begin{tabular}{|c|c|c|c|c|}
\hline Amostra & $\begin{array}{c}\text { Unidade } \\
\text { Litoestratigráfica/Idade }\end{array}$ & Procedência & Espécie & Coleção Científica \\
\hline $107-\operatorname{Tr}$ & Formação Maecuru & Margem do Rio Maecuru & "Dalmanites" tumilobus & UFRJ \\
\hline $110-\operatorname{Tr}$ & Formação Maecuru & Margem do Rio Maecuru & "Palpebrops" goeldi & UFRJ \\
\hline $111-\operatorname{Tr}$ & Formação Maecuru & Margem do Rio Maecuru & "Palpebrops" goeldi & UFRJ \\
\hline $115-\operatorname{Tr}$ & Formação Maecuru & Margem do Rio Maecuru & Amazonaspis maecurua & UFRJ \\
\hline $116-\operatorname{Tr}$ & Formação Maecuru & Margem do Rio Maecuru & "Dalmanites" tumilobus & UFRJ \\
\hline $118-\operatorname{Tr}$ & Formação Maecuru & Margem do Rio Maecuru & "Palpebrops" goeldi & UFRJ \\
\hline $2600-\mathrm{I}$ & Grupo Clinton & Lockport, Estado de New York & $\begin{array}{c}\text { Trimerus } \\
\text { delphinocephalus }\end{array}$ & $\mathrm{MN}$ \\
\hline $2877-\mathrm{I}$ & Formação Ererê & Monte Alegre, PA & "Dalmanites" tumilobus & $\mathrm{MN}$ \\
\hline 2913-I & Formação Maecuru & Margem do Rio Maecuru & ?Homalonotus sp. & MN \\
\hline 3371-I & Formação Maecuru & Margem do Rio Maecuru & "Homalonotus" derbyi & $\mathrm{MN}$ \\
\hline $3372-\mathrm{I}$ & Formação Maecuru & Margem do Rio Maecuru & "Homalonotus" derbyi & $\mathrm{MN}$ \\
\hline 3373-I & Formação Maecuru & Margem do Rio Maecuru & "Homalonotus" derbyi & MN \\
\hline
\end{tabular}




\begin{tabular}{|c|c|c|c|c|}
\hline Amostra & $\begin{array}{c}\text { Unidade } \\
\text { Litoestratigráfica/Idade }\end{array}$ & Procedência & Espécie & Coleção Científica \\
\hline 3374-I & Formação Maecuru & Margem do Rio Maecuru & $\begin{array}{c}\text { "Tarijactinoides" } \\
\text { acanthurus }\end{array}$ & $\mathrm{MN}$ \\
\hline $3375-\mathrm{I}$ & Formação Maecuru & Margem do Rio Maecuru & Phacopina braziliensis & $\mathrm{MN}$ \\
\hline 3376-I & Formação Maecuru & Margem do Rio Maecuru & Phacopina braziliensis & $\mathrm{MN}$ \\
\hline 3377-I & Formação Maecuru & Margem do Rio Maecuru & “Phacops" menurus & $\mathrm{MN}$ \\
\hline 3378-I & Formação Maecuru & Margem do Rio Maecuru & "Phacops" scirpeus & $\mathrm{MN}$ \\
\hline $3379-\mathrm{I}$ & Formação Maecuru & Margem do Rio Maecuru & ?Phacops pullinus & $\mathrm{MN}$ \\
\hline $3380-\mathrm{I}$ & Formação Maecuru & Margem do Rio Maecuru & "Phacops" macropyge & $\mathrm{MN}$ \\
\hline 3381-I & Formação Maecuru & Margem do Rio Maecuru & "Phacops" macropyge & $\mathrm{MN}$ \\
\hline $3389-\mathrm{I}$ & Formação Maecuru & Margem do Rio Maecuru & "Malvinella" australis & $\mathrm{MN}$ \\
\hline 3390-I & Formação Maecuru & Margem do Rio Maecuru & "Dalmanites" galea & $\mathrm{MN}$ \\
\hline 3391-I & Formação Maecuru & Margem do Rio Maecuru & "Dalmanites" tumilobus & $\mathrm{MN}$ \\
\hline 3392-I & Formação Maecuru & Margem do Rio Maecuru & "Dalmanites" tumilobus & $\mathrm{MN}$ \\
\hline 3393-I & Formação Maecuru & Margem do Rio Maecuru & "Dalmanites" tumilobus & $\mathrm{MN}$ \\
\hline 3394-I & Formação Ererê & Monte Alegre, PA & Eldredgeia paituna & $\mathrm{MN}$ \\
\hline 3395-I & Formação Ererê & Monte Alegre, PA & Eldredgeia paituna & $\mathrm{MN}$ \\
\hline DGM-55 & Formação Ponta Grossa & Ponta Grossa, PR & Burmeisteria notica & MCT/DNPM \\
\hline DGM-58 & Formação Ponta Grossa & Ponta Grossa, PR & Burmeisteria notica & MCT/DNPM \\
\hline
\end{tabular}




\begin{tabular}{|c|c|c|c|c|}
\hline Amostra & $\begin{array}{c}\text { Unidade } \\
\text { Litoestratigráfica/Idade }\end{array}$ & Procedência & Espécie & Coleção Científica \\
\hline DGM-63 & Formação Ponta Grossa & Ponta Grossa, PR & Burmeisteria notica & MCT/DNPM \\
\hline DGM-1563 & Formação Ponta Grossa & Ponta Grossa, PR & Burmeisteria notica & MCT/DNPM \\
\hline DGM-1564 & Formação Ponta Grossa & Ponta Grossa, PR & Burmeisteria sp. & MCT/DNPM \\
\hline DGM-1567 & Formação Ponta Grossa & Ponta Grossa, PR & Burmeisteria sp. & MCT/DNPM \\
\hline DGM-1568 & Formação Ponta Grossa & Ponta Grossa, PR & Burmeisteria $\mathrm{sp}$. & MCT/DNPM \\
\hline DGM-1569 & Formação Ponta Grossa & Ponta Grossa, PR & Burmeisteria notica & MCT/DNPM \\
\hline DGM-5010 & Formação Ponta Grossa & Caiapônia, GO & Metacryphaeus australis & MCT/DNPM \\
\hline DGM-5012 & Membro Passagem & Picos, PI & Metacryphaeus meloi & MCT/DNPM \\
\hline DGM-6130 & Formação Pimenteira & Pimenteiras, PI & Burmeisteria sp. & MCT/DNPM \\
\hline DGM-6153 & Membro Passagem & região entre Picos e Oeiras, PI & Metacryphaeus meloi & MCT/DNPM \\
\hline DGM-6155 & Membro Passagem & região entre Picos e Oeiras, PI & Metacryphaeus meloi & MCT/DNPM \\
\hline 169039 & Formação Pimenteira & região de João Costa, PI & Burmeisteria sp. & FUMDHAM \\
\hline
\end{tabular}




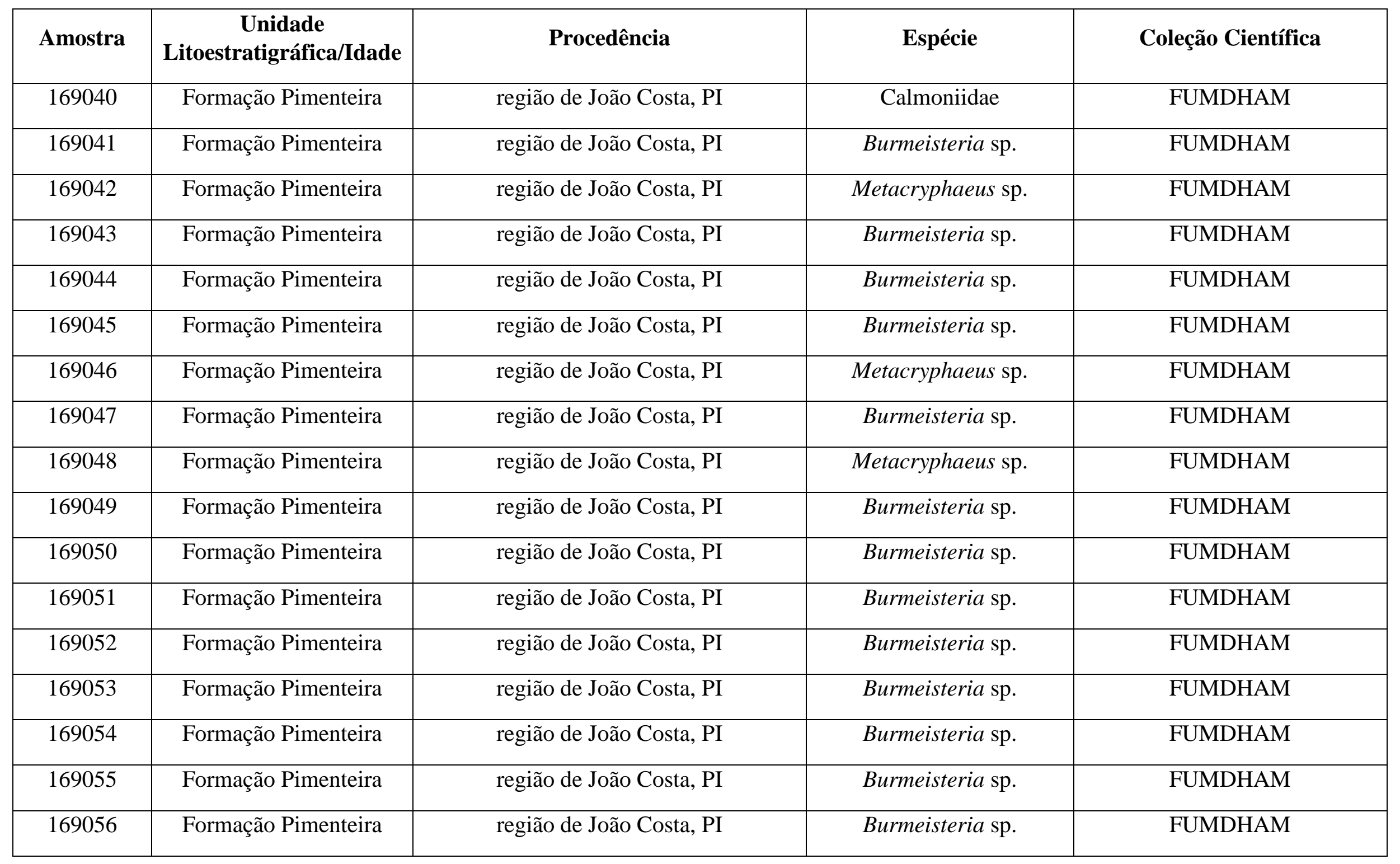




\begin{tabular}{|c|c|c|c|c|}
\hline Amostra & \begin{tabular}{|c|} 
Unidade \\
Litoestratigráfica/Idade
\end{tabular} & Procedência & Espécie & Coleção Científica \\
\hline 197601 & Formação Pimenteira & região de João Costa, PI & $\begin{array}{c}\text { Burmeisteria sp. e } \\
\text { Calmoniidae }\end{array}$ & FUMDHAM \\
\hline 197602 & Formação Pimenteira & região de João Costa, PI & $\begin{array}{l}\text { Burmeisteria sp. e } \\
\text { Metacryphaeus sp. }\end{array}$ & FUMDHAM \\
\hline 197604 & Formação Pimenteira & região de João Costa, PI & Burmeisteria sp. & FUMDHAM \\
\hline LPF-1 & Formação Pimenteira & região de João Costa, PI & Burmeisteria sp. & FUMDHAM \\
\hline LPF-2 & Formação Pimenteira & região de João Costa, PI & Burmeisteria sp. & FUMDHAM \\
\hline LPF-3 & Formação Pimenteira & região de João Costa, PI & Burmeisteria sp. & FUMDHAM \\
\hline LPF-4 & Formação Pimenteira & região de João Costa, PI & Burmeisteria sp. & FUMDHAM \\
\hline LPF-5 & Formação Pimenteira & região de João Costa, PI & Burmeisteria sp. & FUMDHAM \\
\hline LPF-6 & Formação Pimenteira & região de João Costa, $\mathrm{PI}$ & Burmeisteria sp. & FUMDHAM \\
\hline $\begin{array}{c}\text { MKMP - } \\
1489\end{array}$ & Devoniano & Chacoma - Patacamaya & Dipleura dekayi & Museo Noel Kempff Mercado \\
\hline $\begin{array}{c}\text { MKMP - } \\
3604\end{array}$ & Devoniano & & Dipleura dekayi & Museo Noel Kempff Mercado \\
\hline $\begin{array}{c}\text { MKMP - } \\
1483\end{array}$ & Devoniano & Chacoma - Patacamaya & Dipleura dekayi & Museo Noel Kempff Mercado \\
\hline $\begin{array}{c}\text { MKMP - } \\
1478\end{array}$ & Devoniano & Chacoma - Patacamaya & Dipleura dekayi & Museo Noel Kempff Mercado \\
\hline MKMP - & Devoniano & Chacoma - Patacamaya & Dipleura dekayi & Museo Noel Kempff Mercado \\
\hline
\end{tabular}




\begin{tabular}{|c|c|c|c|c|}
\hline 1472 & & & & \\
\hline Amostra & $\begin{array}{c}\text { Unidade } \\
\text { Litoestratigráfica/Idade }\end{array}$ & Procedência & Espécie & Coleção Científica \\
\hline $\begin{array}{c}\text { MKMP - } \\
1470\end{array}$ & Devoniano & Chacoma - Patacamaya & Dipleura dekayi & Museo Noel Kempff Mercado \\
\hline $\begin{array}{c}\text { MKMP - } \\
1465\end{array}$ & Devoniano & Chacoma - Patacamaya & Dipleura dekayi & Museo Noel Kempff Mercado \\
\hline $\begin{array}{l}\text { MKMP - } \\
1488\end{array}$ & Devoniano & Chacoma - Patacamaya & Dipleura dekayi & Museo Noel Kempff Mercado \\
\hline $\begin{array}{c}\text { MKMP - } \\
1482\end{array}$ & Devoniano & Chacoma - Patacamaya & Dipleura dekayi & Museo Noel Kempff Mercado \\
\hline $\begin{array}{l}\text { MKMP - } \\
1496\end{array}$ & Devoniano & Chacoma - Patacamaya & Dipleura dekayi & Museo Noel Kempff Mercado \\
\hline $\begin{array}{c}\text { MKMP - } \\
1471\end{array}$ & Devoniano & Chacoma - Patacamaya & Dipleura dekayi & Museo Noel Kempff Mercado \\
\hline $\begin{array}{l}\text { MKMP - } \\
2649\end{array}$ & Devoniano & Chacoma - Patacamaya & Dipleura dekayi & Museo Noel Kempff Mercado \\
\hline $\begin{array}{c}\text { MKMP - } \\
1740\end{array}$ & Devoniano & Chacoma - Patacamaya & Homalonotidae & Museo Noel Kempff Mercado \\
\hline $\begin{array}{l}\text { MKMP - } \\
2251\end{array}$ & Devoniano & Chacoma - Patacamaya & Dipleura dekayi & Museo Noel Kempff Mercado \\
\hline $\mathrm{S} / \mathrm{N}$ & Fm. Iquiri & Km 63 & Dipleura dekayi & Museo Noel Kempff Mercado \\
\hline MKMP - & Devoniano & Chacoma - Patacamaya & Dipleura dekayi & Museo Noel Kempff Mercado \\
\hline
\end{tabular}




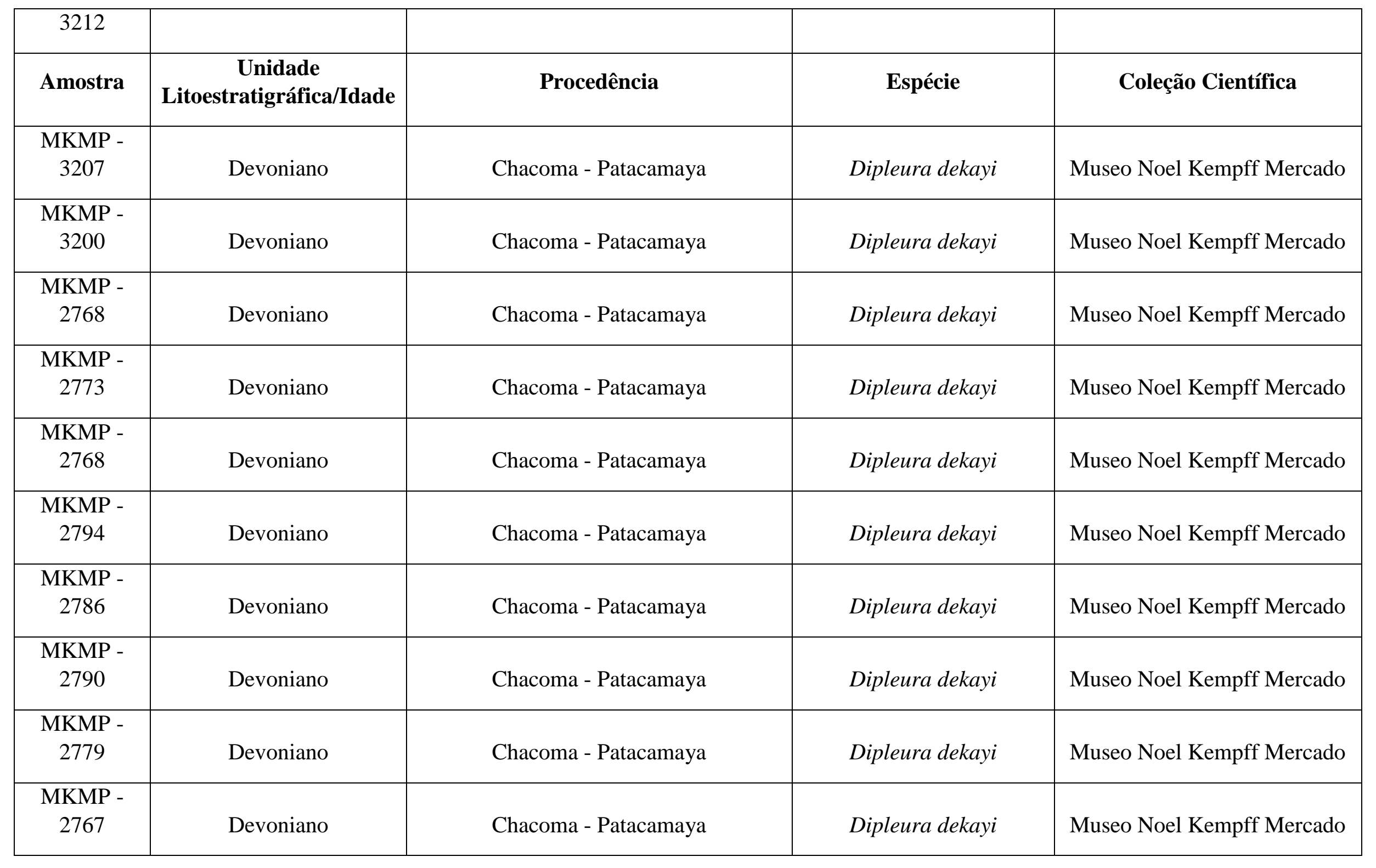




\begin{tabular}{|c|c|c|c|c|}
\hline Amostra & $\begin{array}{c}\text { Unidade } \\
\text { Litoestratigráfica/Idade }\end{array}$ & Procedência & Espécie & Coleção Científica \\
\hline $\begin{array}{l}\text { MKMP - } \\
2742\end{array}$ & Devoniano & Chacoma - Patacamaya & Dipleura dekayi & Museo Noel Kempff Mercado \\
\hline $\begin{array}{c}\text { MKMP - } \\
3800\end{array}$ & Devoniano & Chacoma - Patacamaya & Dipleura dekayi & Museo Noel Kempff Mercado \\
\hline $\begin{array}{c}\mathrm{MKMP}- \\
2758\end{array}$ & Devoniano & Chacoma - Patacamaya & Dipleura dekayi & Museo Noel Kempff Mercado \\
\hline $\begin{array}{c}\text { MKMP - } \\
3602\end{array}$ & Devoniano & Chacoma - Patacamaya & Dipleura dekayi & Museo Noel Kempff Mercado \\
\hline $\begin{array}{l}\text { MKMP - } \\
2743\end{array}$ & Devoniano & Chacoma - Patacamaya & Dipleura dekayi & Museo Noel Kempff Mercado \\
\hline $\mathrm{S} / \mathrm{N}$ & Devoniano & Chaurumani - Patacamaya & M. giganteus & Museo Noel Kempff Mercado \\
\hline $\begin{array}{l}\text { MKMP - } \\
3692\end{array}$ & Devoniano & & M. giganteus & Museo Noel Kempff Mercado \\
\hline $\begin{array}{l}\text { MKMP - } \\
887\end{array}$ & Devoniano & & M. giganteus & Museo Noel Kempff Mercado \\
\hline $\begin{array}{c}\text { MKMP - } \\
271\end{array}$ & Devoniano & & M. giganteus & Museo Noel Kempff Mercado \\
\hline
\end{tabular}




\begin{tabular}{|c|c|c|c|c|}
\hline Amostra & $\begin{array}{c}\text { Unidade } \\
\text { Litoestratigráfica/Idade }\end{array}$ & Procedência & Espécie & Coleção Científica \\
\hline $\begin{array}{l}\text { MKMP - } \\
294\end{array}$ & Devoniano & & M. giganteus & Museo Noel Kempff Mercado \\
\hline $\begin{array}{l}\text { MKMP - } \\
006\end{array}$ & Devoniano & & M. tuberculatus & Museo Noel Kempff Mercado \\
\hline $\begin{array}{l}\text { MKMP - } \\
988\end{array}$ & Devoniano & & M. tuberculatus & Museo Noel Kempff Mercado \\
\hline $\begin{array}{l}\text { MKMP - } \\
003\end{array}$ & Devoniano & & M. tuberculatus & Museo Noel Kempff Mercado \\
\hline $\begin{array}{l}\text { MKMP - } \\
307\end{array}$ & Devoniano & & M. tuberculatus & Museo Noel Kempff Mercado \\
\hline $\begin{array}{c}\text { MKMP - } \\
367\end{array}$ & Devoniano & & M. tuberculatus & Museo Noel Kempff Mercado \\
\hline $\begin{array}{l}\text { MKMP - } \\
985\end{array}$ & Devoniano & & M. tuberculatus & Museo Noel Kempff Mercado \\
\hline $\begin{array}{l}\text { MKMP - } \\
992\end{array}$ & Devoniano & & M. tuberculatus & Museo Noel Kempff Mercado \\
\hline MKMP - & Devoniano & & M. tuberculatus & Museo Noel Kempff Mercado \\
\hline
\end{tabular}




\begin{tabular}{|c|c|c|c|c|}
\hline 2231 & & & & \\
\hline Amostra & $\begin{array}{c}\text { Unidade } \\
\text { Litoestratigráfica/Idade }\end{array}$ & Procedência & Espécie & Coleção Científica \\
\hline $\begin{array}{c}\text { MKMP - } \\
3935\end{array}$ & Devoniano & & M. tuberculatus & Museo Noel Kempff Mercado \\
\hline $\begin{array}{c}\text { MKMP - } \\
017\end{array}$ & Devoniano & & M. tuberculatus & Museo Noel Kempff Mercado \\
\hline $\begin{array}{c}\text { MKMP - } \\
2201\end{array}$ & Devoniano & & M. tuberculatus & Museo Noel Kempff Mercado \\
\hline $\begin{array}{c}\text { MKMP - } \\
783\end{array}$ & Devoniano & & M. tuberculatus & Museo Noel Kempff Mercado \\
\hline $\begin{array}{c}\text { MKMP - } \\
3850\end{array}$ & Devoniano & & M. tuberculatus & Museo Noel Kempff Mercado \\
\hline $\begin{array}{c}\text { MKMP - } \\
538\end{array}$ & Devoniano & & M. tuberculatus & Museo Noel Kempff Mercado \\
\hline $\begin{array}{c}\text { MKMP - } \\
872\end{array}$ & Devoniano & & M. tuberculatus & Museo Noel Kempff Mercado \\
\hline $\begin{array}{c}\text { MKMP - } \\
618\end{array}$ & Devoniano & & M. tuberculatus & Museo Noel Kempff Mercado \\
\hline $\begin{array}{c}\text { MKMP - } \\
791\end{array}$ & Devoniano & & M. tuberculatus & Museo Noel Kempff Mercado \\
\hline $\begin{array}{l}\text { MKMP - } \\
550\end{array}$ & Devoniano & & M. tuberculatus & Museo Noel Kempff Mercado \\
\hline
\end{tabular}




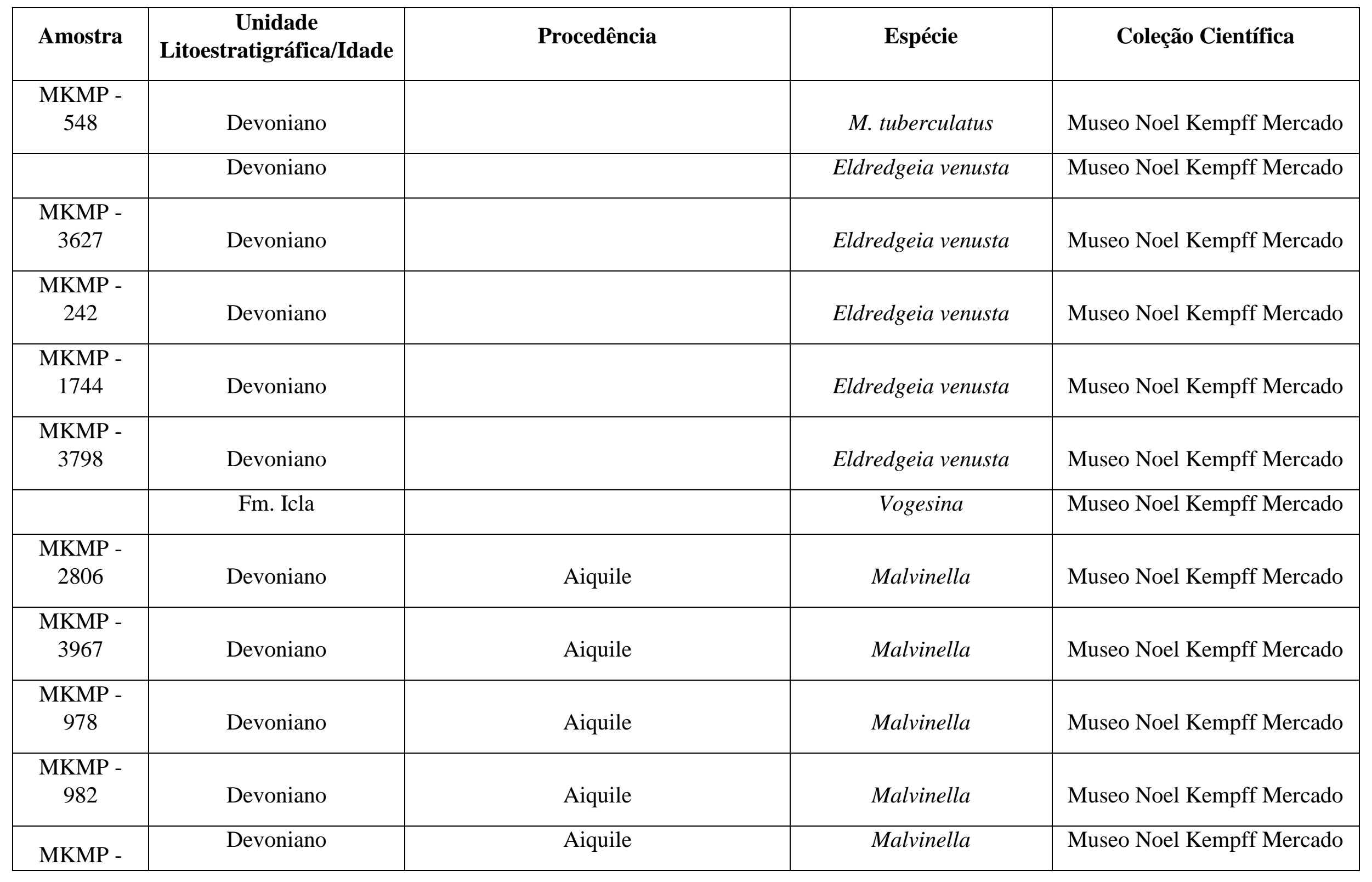




\begin{tabular}{|c|c|c|c|c|}
\hline 996 & & & & \\
\hline Amostra & $\begin{array}{c}\text { Unidade } \\
\text { Litoestratigráfica/Idade }\end{array}$ & Procedência & Espécie & Coleção Científica \\
\hline $\begin{array}{c}\text { MKMP - } \\
1428\end{array}$ & Devoniano & Aiquile & Malvinella & Museo Noel Kempff Mercado \\
\hline MKMP -996 & Devoniano & Aiquile & Malvinella & Museo Noel Kempff Mercado \\
\hline $\begin{array}{c}\text { MKMP - } \\
2315\end{array}$ & Devoniano & Aiquile & Malvinella & Museo Noel Kempff Mercado \\
\hline $\begin{array}{c}\text { MKMP - } \\
1723\end{array}$ & Devoniano & Aiquile & Malvinella & Museo Noel Kempff Mercado \\
\hline $\begin{array}{c}\text { MKMP - } \\
3507\end{array}$ & Devoniano & Aiquile & Malvinella & Museo Noel Kempff Mercado \\
\hline $\begin{array}{l}\text { MKMP - } \\
769\end{array}$ & Devoniano & Aiquile & Malvinella & Museo Noel Kempff Mercado \\
\hline MKMP - & Devoniano & Aiquile & Malvinella & Museo Noel Kempff Mercado \\
\hline
\end{tabular}




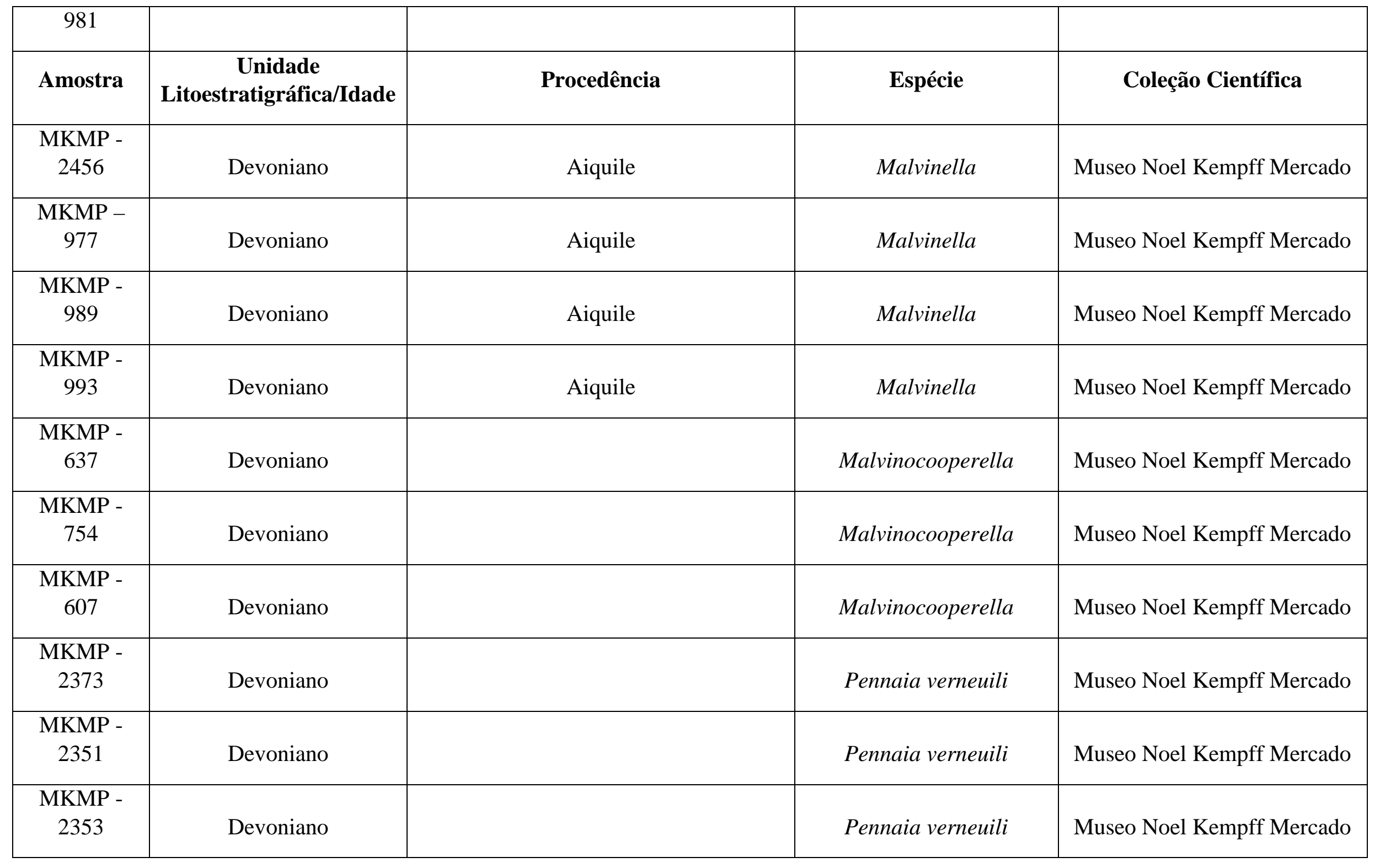




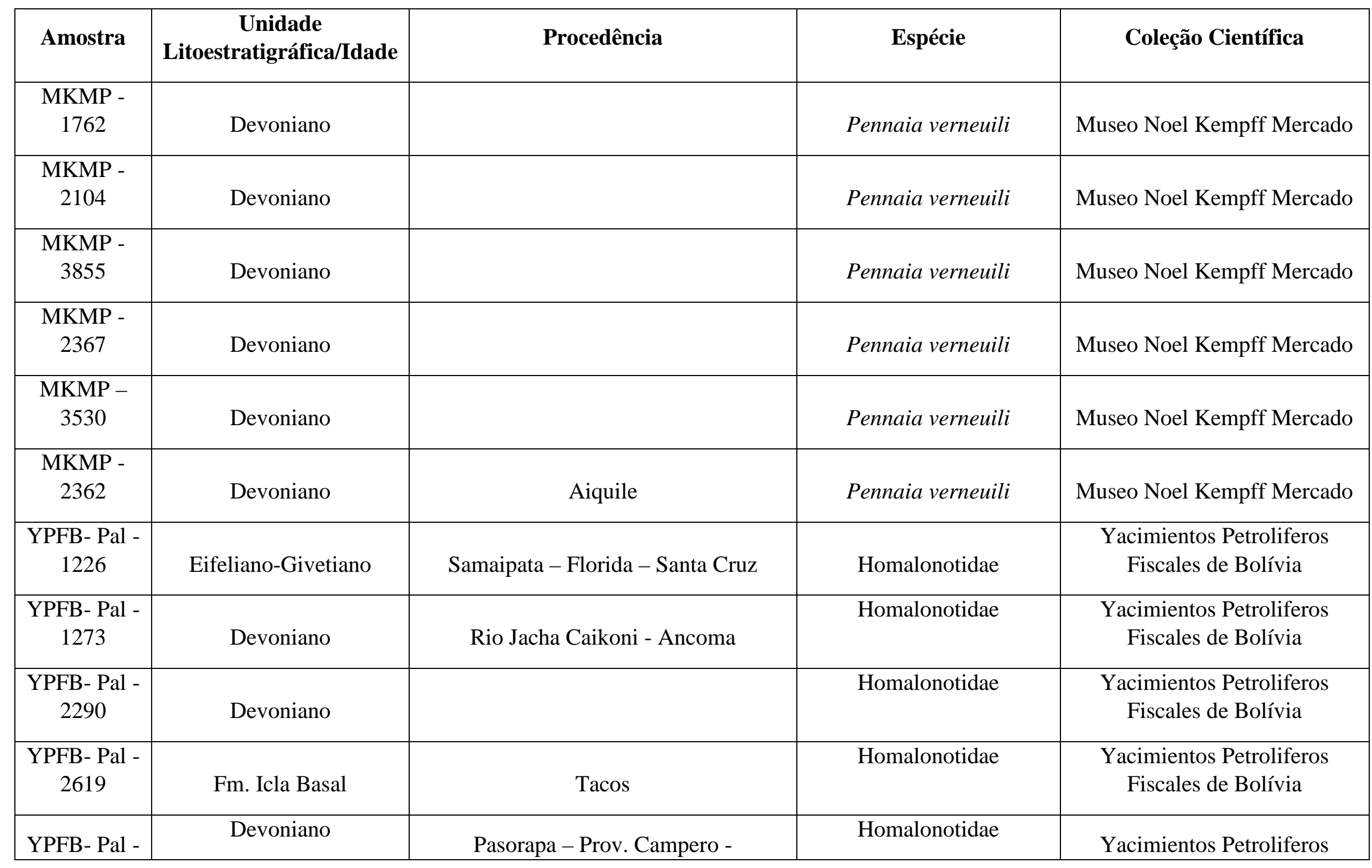




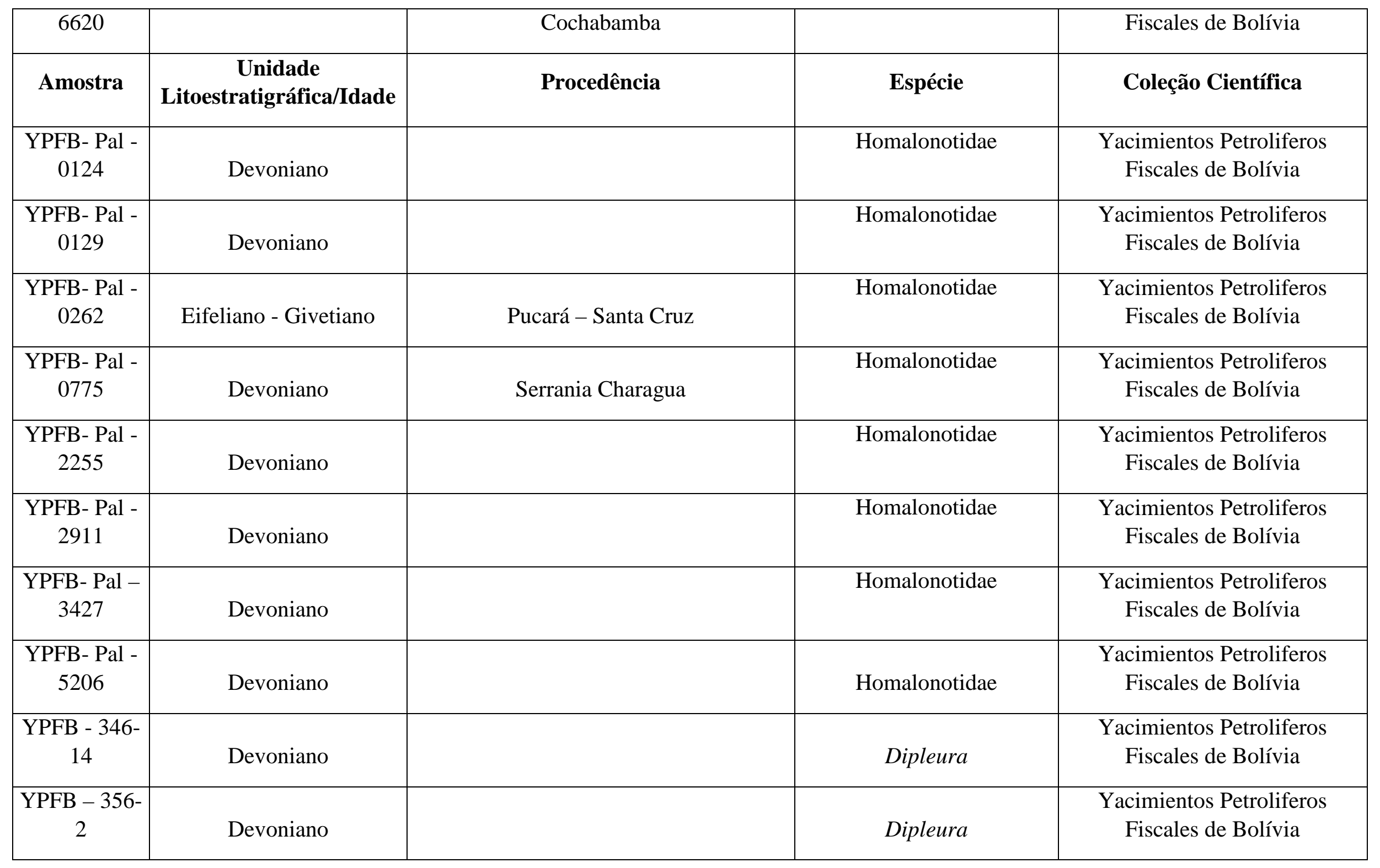




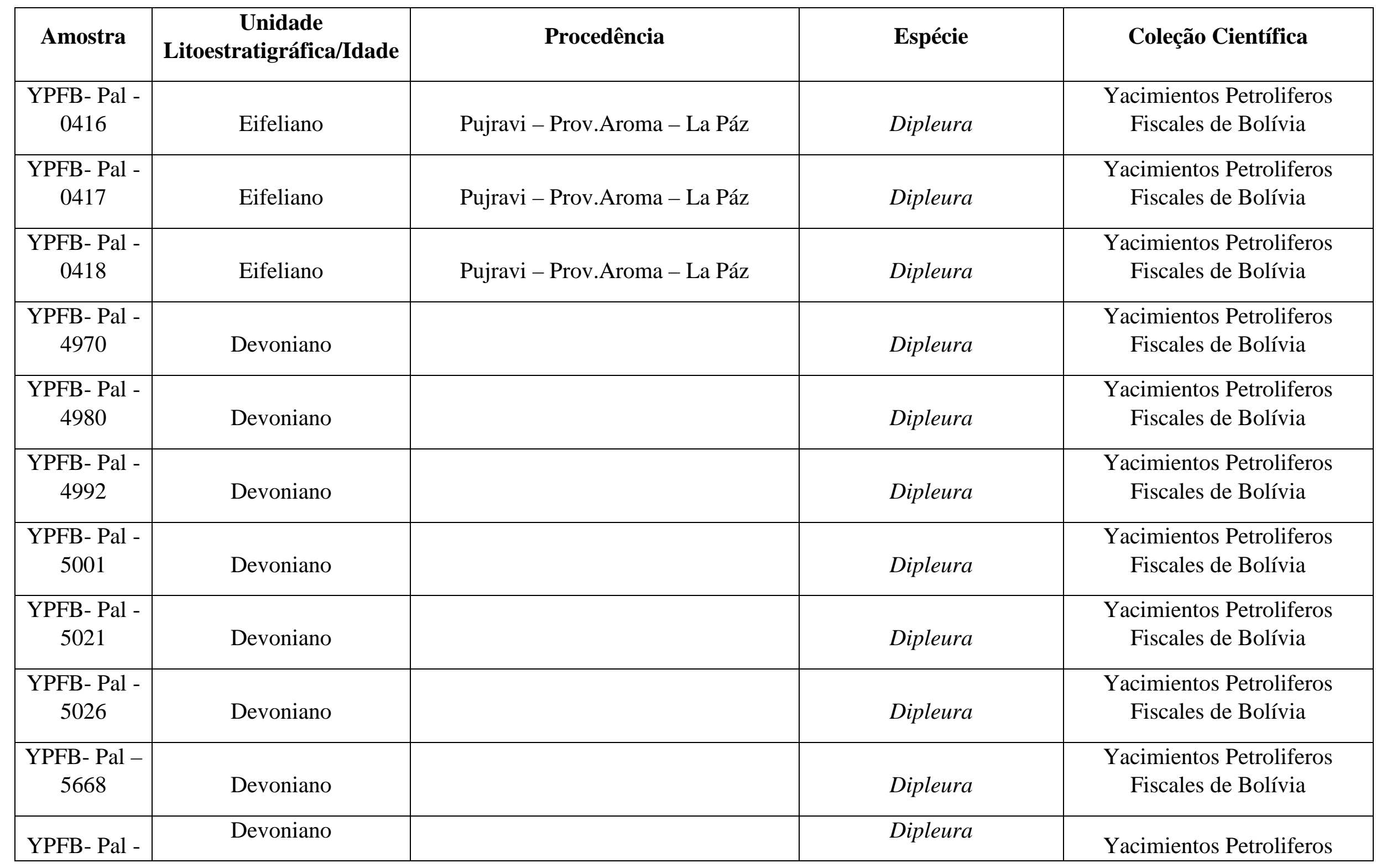




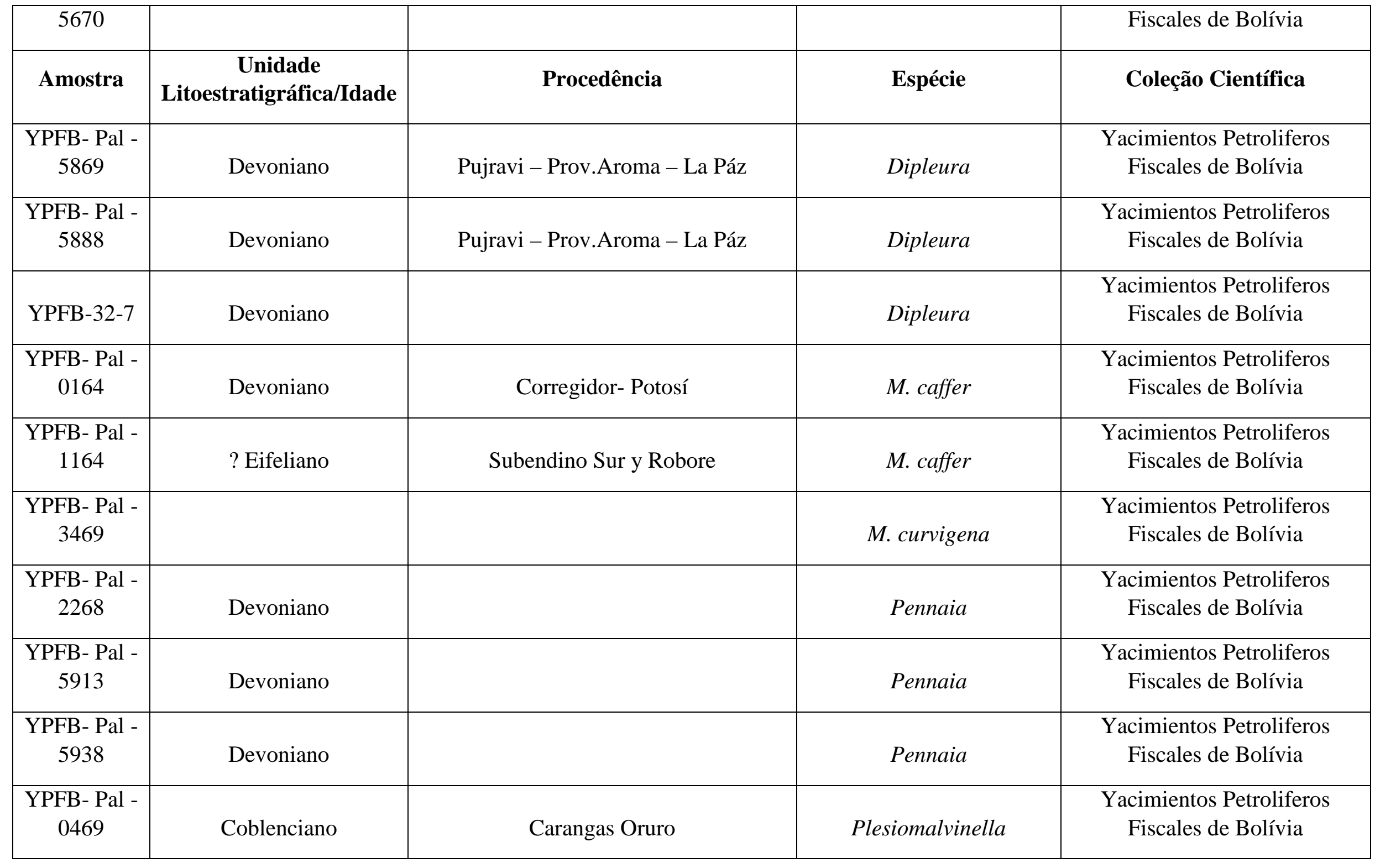




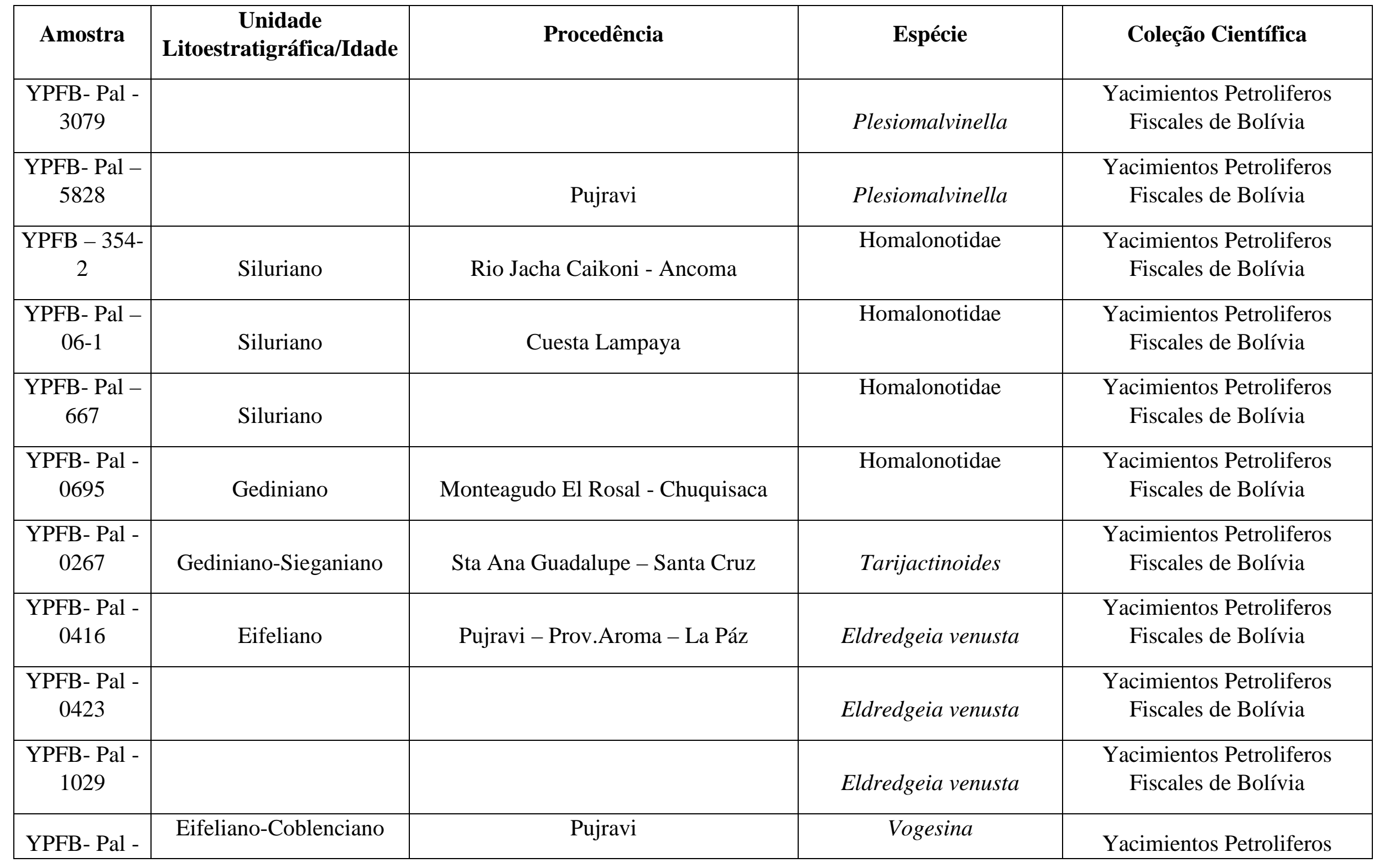


ANEXO II

THE “METACRYPHAEUS TUBERCULATUS GROUP” (TRILOBITA, CALMONIIDAE) FROM THE DEVONIAN OF THE PARNAÍBA BASIN, BRAZIL (in press)

doi: 10.5710/AMGH.03.12.2015.2924 


\section{Ameghiniana MS2924}

3 messages

Nestor Toledo <ntoledo.biol@gmail.com>

To: fvemeira@gmail.com

Tue, May 10, 2016 at 11:14 AM

\section{Dear Author}

My name is Néstor Toledo and I am member of the Editorial Production Team of Ameghiniana. Your manuscript has been forwarded to our team after being accepted by the Academic Editor. I will be in contact with you during the production editorial process. During this process we will perform a copyedit revision of the manuscript to make sure the format of the text and figures meets the guidelines and requirements of our journal. Once this revision is finished we will be in touch again for correcting the galley proofs.

I would like also to send you the Copyright Release Form that you should complete, sign, and send via postal mail to the Asociación Paleontológica Argentina (digital versions are not accepted).

Finally, I would like to let you know that a preprint version of your manuscript is already available online at the Ameghiniana website (in the FUTURE ISSUES section). Your manuscript has a registered DOI so it can be cited if necessary.

Please let me know if you have any questions regarding your manuscript.

Best regards,

Néstor Toledo

Ameghiniana

Editorial Production Team

国 Copyright 2924.docx

$79 \mathrm{~K}$

Nestor Toledo <ntoledo.biol@gmail.com>

To: fvemeira@gmail.com

Tue, May 17, 2016 at 9:12 PM

Dear Author,

I'm sending the corrected version of your manuscript. Please provide the requested clarifications of citations and (especially) attend to those phrases corrected by our english corrector, in order to ensure they've preserved the original sense-

I'll contact again you very soon

Best regards,

Néstor Toledo

[Quoted text hidden] 
THE “METACRYPHAEUS TUBERCULATUS GROUP” (TRILOBITA, CALMONIIDAE) FROM THE DEVONIAN OF THE PARNAÍBA BASIN, BRAZIL.

\section{EL “GRUPO METACRYPHAEUS TUBERCULATUS” (TRILOBITA, CALMONIIDAE) DEL DEVÓNICO DE LA CUENCA DEL PARNAÍbA, BRASIL.}

F. van. E. Meira ${ }^{1}$, F. B. Carbonaro ${ }^{2}$. R. P. Ghilardi ${ }^{3}$, J. de M. Leme ${ }^{4}$

1 Programa de Pós-graduação em Geoquímica e Geotectônica, Instituto de Geociências, Universidade de São Paulo - USP. Rua do Lago, 562, CEP 05508-080, São Paulo/SP. Brasil.

${ }^{2}$ Programa de Pós-graduação em Biologia Comparada, Faculdade de Filosofia, Ciências e Letras de Ribeirão Preto, Universidade de São Paulo - USP. Av. Bandeirantes, 3900, CEP 14040-900, Ribeirão Preto/ SP, Brasil.

${ }^{3}$ Departamento de Ciências Biológicas, Faculdade de Ciências de Bauru, Universidade Estadual Paulista Júlio de Mesquita Filho - UNESP. Av. Eng. Luiz Edmundo Carrijo Coube, 14-01, CEP 17033-360, Bauru/SP, Brasil.

${ }^{4}$ Departamento de Geologia Sedimentar e Ambiental, Instituto de Geociências, Universidade de São Paulo - USP. Rua do Lago, 562, CEP 05508-080, São Paulo/SP. Brasil.

*Corresponding author: fvemeira@gmail.com 
Abstract. The calmoniid trilobites of the "Metacryphaeus tuberculatus group" occur in many Devonian localities within the Malvinokaffric Realm. In the Parnaíba Basin, in the northeast of Brazil, the group is represented by the species Metacryphaeus tuberculatus (Kozlowski, 1923) and Metacryphaeus meloi (Carvalho et al., 1997), the former from the late Eifelian-early Givetian Pimenteira Formation in the Picos city area, and the latter from the early Givetian Passagem Member (Pimenteira Formation) from Picos and Pimenteiras cities vicinities, in the Piauí State. The present study reinforces the occurrence of M. tuberculatus in the Pimenteira Formation based on specimens found in the region of João Costa city, southeastern Piauí State and reports the presence of genal spines in some individuals of M. meloi, indicating a feature previously unknown to this species.

Keywords. Metacryphaeus tuberculatus, Metacryphaeus meloi, Pimenteira Formation, Devonian, Parnaíba Basin.

Resumen. Los trilobites calmónidos del "Grupo Metacryphaeus tuberculatus" se producen em diversas localidades del Devónico em el Dominio Malvinocáfrico. En la Cuenca del Parnaíba, en el noreste de Brasil, el grupo está representado por las especies Metacryphaeus tuberculatus (Kozlowski, 1923) y Metacryphaeus meloi (Carvalho et al., 1997), la primera del NeoeifelianoEogivetiano de la Formacíon Pimenteira en el área de la ciudad de Picos, y la segunda del Eogivetiano del Miembro Passagem (Formacíon Pimenteira), de las proximidades de las ciudades de Picos y Pimenteiras, en el Estado del Piauí. El presente estudio refuerza la presencia de M. tuberculatus en la Formación Pimenteira basado en especímenes encontrados en la región de la ciudad de João Costa, en el sureste del Estado de Piaú, y informa la presencia de espinas genales en algunos individuos de $M$. meloi, indicando una característica previamente desconocida esta especie.

Palavras clave. Metacryphaeus tuberculatus, Metacryphaeus meloi, Formación Pimenteira, Devónico, Cuenca del Parnaíba. 


\section{INTRODUCTION}

THE genus Metacryphaeus (Reed, 1907) is the most typical taxon of the calmoniid trilobites, with several species occurring in Devonian deposits in many localities of South America plus South Africa. In Brazil, it is long known from the Paraná Basin with the species Metacryphaeus australis (Clarke, 1913). A second species, M. rotundatus (Kozlowski, 1923), originally recorded from Bolivia, was recently incorporated to the list of Metacryphaeus species in this basin (Soares et al., 2008). In the Parnaíba Basin, located in northeast of Brazil, the species Metacryphaeus kegeli (Carvalho et al., 1997) and Metacryphaeus meloi (Carvalho et al., 1997) occur in the Pimenteira Formation, and rare fragments of an indeterminate species of Metacryphaeus were collected from the Longá Formation (Carvalho, 1995). Claims that Metacryphaeus tuberculatus (Kozlowski, 1923), formerly known from Bolivia, would also be present in the Parnaíba Basin were raised by Lieberman et al. (1991) and Lieberman (1993), based on a specimen found in the Pimenteira Formation in the area of Picos city, in the east part of Piauí State. These statements were subsequently questioned by Carvalho et al. (1997), who contested the specimen provenance.

The present study adds some new information concerning the members of the "Metacryphaeus tuberculatus group" (Lieberman, 1993) from the Pimenteira Formation. It endorses the claims that M. tuberculatus would be present in the Pimenteira Formation with the discovery of specimens ascribed to this taxon from the São João Vermelho village, in João Costa city, southeastern Piauí State, and reports the presence of genal spines in some specimens of M. meloi, a feature not known for this species.

The occurrences of M. meloi cover different areas from the eastern Piauí State. M. tuberculatus is known only from Picos and João Costa areas (Fig. 1). 


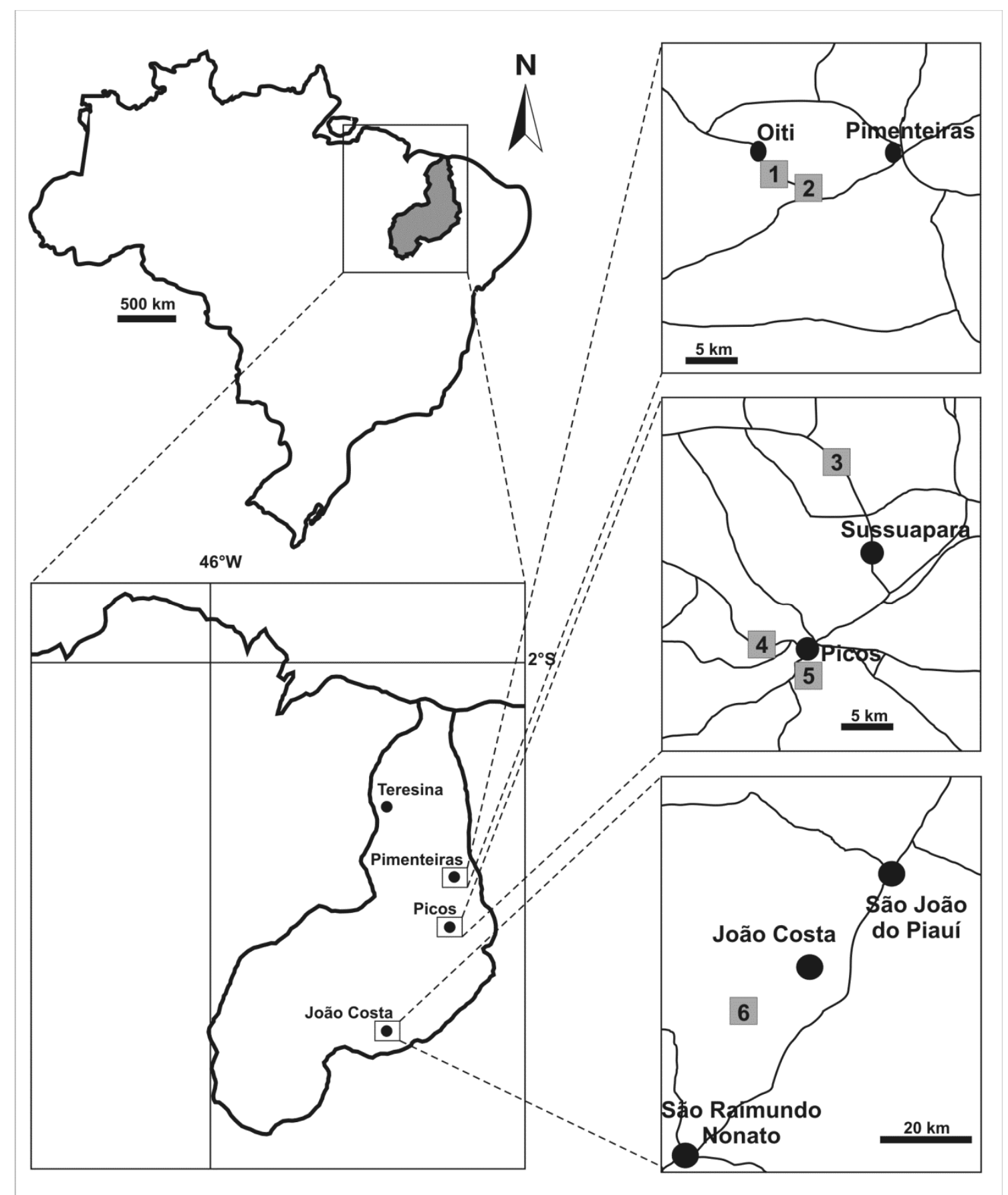

Figure 1. Areas of documented occurrences of Metacryphaeus meloi (1-4) and Metacryphaeus tuberculatus $(5,6)$ in the Piauí State, northeastern Brazil. 1, Rio Banguê, Pimenteiras city (Ponciano et al., 2012a); 2, Oiti, Pimenteiras city; 3, Barreiro Branco, Sussuapara city; 4, km 305 of BR 316, Picos city (Carvalho et al., 1997; Ponciano et al., 2012a); 5, 2 km south of Picos city (Lieberman et al., 1991); 6, São João Vermelho, João Costa city (present work). Modified from Ponciano et al. (2012a, b). 


\section{GEOLOGICAL SETTING}

The Middle Devonian to Early Carboniferous interval in the Parnaíba Basin is represented by Pimenteira, Cabeças and Longá formations, part of the Canindé Group.

The Parnaíba sedimentary basin corresponds to the south-central portion of Meio Norte sedimentary Province (Góes, 1995), also known as Parnaíba Province (Almeida et al., 1977). The province is bounded to the north by the Vicente Ferrer-Urbano Santos-Guamá Arc; to the east, the fault Taua; to the southeast by Lineament Senador Pompeu; and to the west, by Lineament Tocantins-Araguaia basin, and northwest at Tocantins Arc (Góes, 1995).

The Pimenteira Formation, dated between the late Eifelian and early Givetian on the Parnaíba Basin eastern flank (Melo, 1985; Grahn et al., 2006), but extending from the late Eifelian to early Famennian on central portion and western flank (Grahn et al., 2006, 2008), contacts both the underlain Itaim Formation and the overlain Cabeças Formation (Vaz et al., 2007). Its lithology consists of rhythmites of fine sandstones with hummocky cross-stratification and bioturbed pelites (Vaz et al., 2007; Fonseca and Ponciano, 2011), represented shallow platform environments dominated by storms (Della Fávera, 1990). The variety of fossil consists of many invertebrates (brachiopods, pelecypods, gastropods, tentaculitids, trilobites, crinoids, conularids, ostracods and hyolithids), vertebrates (chondrichthyes and acanthodians), and plants (Kegel 1953, Fonseca and Melo, 1987; Melo, 1985; Carvalho, 1995; Caputo et al., 2005). According to Melo (1988), in the range of outcrops located east of the basin, the fossiliferous horizons occur mainly at the base of sandstones with hummocky cross-stratification associated with intraclasts of clay or quartz pebbles and within ferruginous concretions located on the top of the outcrop section.

The Passagem Member corresponds to a proximal facies of the upper Pimenteira Formation Passagem Member, cropping out on the eastern flank of Parnaíba Basin (Ponciano et al., 2010), dated from the Givetian based on the invertebrate macrofossils present (Melo, 1988). It consists mainly of fine sandstones with asymptotic and hummocky cross-stratification, with subordinate siltstone interbeds (Ponciano et al., 2010). Fossils consist in invertebrates (brachiopods, peleypods, gastropods, trilobites, crinoids and tentaculitids) and plants, occurring in yellowish to purplish fine-grained sandstones very micaceous interbedded with siltstone and conglomeratic sandstones (Fonseca and Ponciano, 2011). 
Initially proposed as the basal unit of the Cabeças Formation (Plummer, 1948), Beurlen (1965) observed this unit laterally gradating to facies typical of the upper Pimenteira Formation in the Picos region. Recent studies have confirmed Beurlen's (1965) statement (Breuer and Grahn, 2011; Ponciano et al., 2012).

\section{MATERIAL AND METHODS}

The studied material consists of external and internal molds preserved in sandstones and concretions. Specimens are housed at Fundação Museu do Homem Americano (FUNDHAM), Museu de Ciências da Terra of the Departamento Nacional de Produção Mineral (MCTDNPM), American Museum of Natural History (AMNH), Instituto de Geociências of the Universidade Federal do Rio de Janeiro (IG-UFRJ) and Museu Paraense Emílio Goeldi (MPEG).

The systematic review of the material followed the criteria and morphological terminology used in Harrington et al. (1959), Eldredge and Braniša (1980), Lieberman et al. (1991), Lieberman (1993) and Whittington et al. (1997). For photographs, some samples were coated with magnesium oxide in order to highlight details (Figs.2.2-3).

Abbreviations. exsag, exsagittally.

\section{RESULTS}

\section{SYSTEMATIC PALEONTOLOGY}

Order PHACOPIDA Salter, 1864

Family CALMONIIDAE Delo, 1935

Subfamily CALMONIINAE Delo, 1935

Genus Metacryphaeus Reed, 1907

Type species. Metacryphaeus caffer Salter, 1856

Metacryphaeus tuberculatus Kozlowski, 1923 
Figures 2.1-5

1923. Cryphaeus australis var. tuberculatus Kozlowski, p. 43, pl. 3, figs. 20-21.

?1923. Cryphaeus australis Kozlowski, p. 43, pl. 3, fig. 7.

1965. Metacryphaeus caffer var. Braniša, p. 106, pl. 21, figs. 14, 16.

1968. Metacryphaeus tuberculatus Wolfart, p. 102, pl. 17, fig. 17, pl. 18, fig. 1.

1991. Metacryphaeus tuberculatus Lieberman et al., p. 825-827, fig. 1.1-1.6, 1.8, 1.9, 1.11-

1.13 .

1993. Metacryphaeus tuberculatus Lieberman, p. 562, fig. 2.11.

Diagnosis (Lieberman, 1993). Coarsely tuberculose prosopon, broad and triangular extension of cephalic anterior border beyond glabella; rounded frontal lobe; deep, broad, strongly divergent cephalic axial furrows.

Examined material. FUMDHAM 197602; AMNH 63283.

Geographic and stratigraphic provenance. Late Eifelian to early Givetian. Pimenteira Formation at São João Vermelho village, João Costa city, southeastern Piaúi State; also from 2 km south of Picos city, eastern Piauí State, Brazil.

Description (FUMDHAM 197602). Cephalon with subtriangular outline, length (sag.) about $55 \%$ the width (tr.). Anterior margin with moderate convexity. Lateral margins slightly convex. Posterior border furrow straight and broad (exsag.), with the same length (exsag.) as the occipital furrow. Narrow occipital ring (sag., exsag.) medially vaulted forward. Cephalon covered by small tubercles.

Glabellar lobes L1 narrow (exsag.) crescent in shape, curved backward, in contact with the axial furrows. Glabellar lobes L2 wider (exsag.), crescent in shape and curved backward. Glabellar lobes L3 straight, broadening abaxially. Frontal lobe LA extended laterally, with subrhomboidal outline and length (sag.) about $60 \%$ the glabella length. Axial furrows broad.

Occipital furrow S0 wide (sag., exsag.) in contact with axial furrows, bearing apodemal pits close to the limit with axial furrows. Glabellar furrows S1 crescent in shape, with apodemal pits. Glabellar furrows S2 shallow, slightly vaulted posteriorly, in contact with axial furrows. Glabellar furrows L3 broad (exsag.), oblique and straight, in contact with axial furrows. 
Thorax composed of 11 segments. Axial rings with width (tr.) about $40 \%$ the maximum breadth of thoracic segments. Axial rings with width (sag., exsag.) constant through their extension, with slight bulging abaxially. Pleurae with deep and broad pleural furrows. Anterior pleural band slightly elevated, bearing a row of small tubercles well delineated on the surface. Posterior pleural band wider (exsag.) and more elevated, with a row of less conspicuous but larger tubercles on it. On the axial rings, tubercles are only observed abaxially.

Pygidium with the first axial rings arched forward; this curving decreases toward the posterior rings. Axial rings with constant width (sag., exsag.) through their extension, widening abaxially. Apodemal pits present abaxially on the ring furrows. Pleural ribs narrowing adaxially. Pleural rib tips slightly vaulted forward in the first ribs; the curvature increases along the posterior ribs.

Discussion. The occurrence of $M$. tuberculatus in the Pimenteira Formation was claimed by Lieberman et al. (1991) and Lieberman (1993), based on a specimen (Fig. 2.1) from $2 \mathrm{~km}$ south of Picos city, eastern Piauí State, differing from Bolivian M. tuberculatus only in having longer (exsag.) lateral glabellar lobes L3 and eyes closer to the genal margin (Lieberman et al., 1991). Carvalho $(1995,1999)$ and Carvalho et al. (1997) questioned the origin of the specimen, alleging that the concretion where it is preserved differs from what is known for the Pimenteira Formation. The comparison of this sample with concretions containing fossils of the homalonotid Burmeisteria notica (Clarke, 1913) from the Pimenteira Formation from Picos city, housed at the Instituto de Geociências of the Universidade de São Paulo (IG-USP) (GP-1E 3/543, GP-1E 4971, GP-1E 5016), reveals similarity in the color and overall aspect, indicating that M. tuberculatus sample of Lieberman et al. (1991) from the Pimenteira Formation might belong to this unit.

Additionally, the analysis of samples from the Pimenteira Formation in the area of João Costa, southeastern Piauí State (Figs.2.2-5), suggests they may belong to M. tuberculatus, as they exhibit some characters typical of $M$. tuberculatus, as the prosopon covered with coarse tubercles and cephalic axial furrows broad and strongly divergent. These specimens from the southeastern Piauí State reinforce previous reports on this species in the Parnaíba Basin (Lieberman et al., 1991; Lieberman, 1993). 


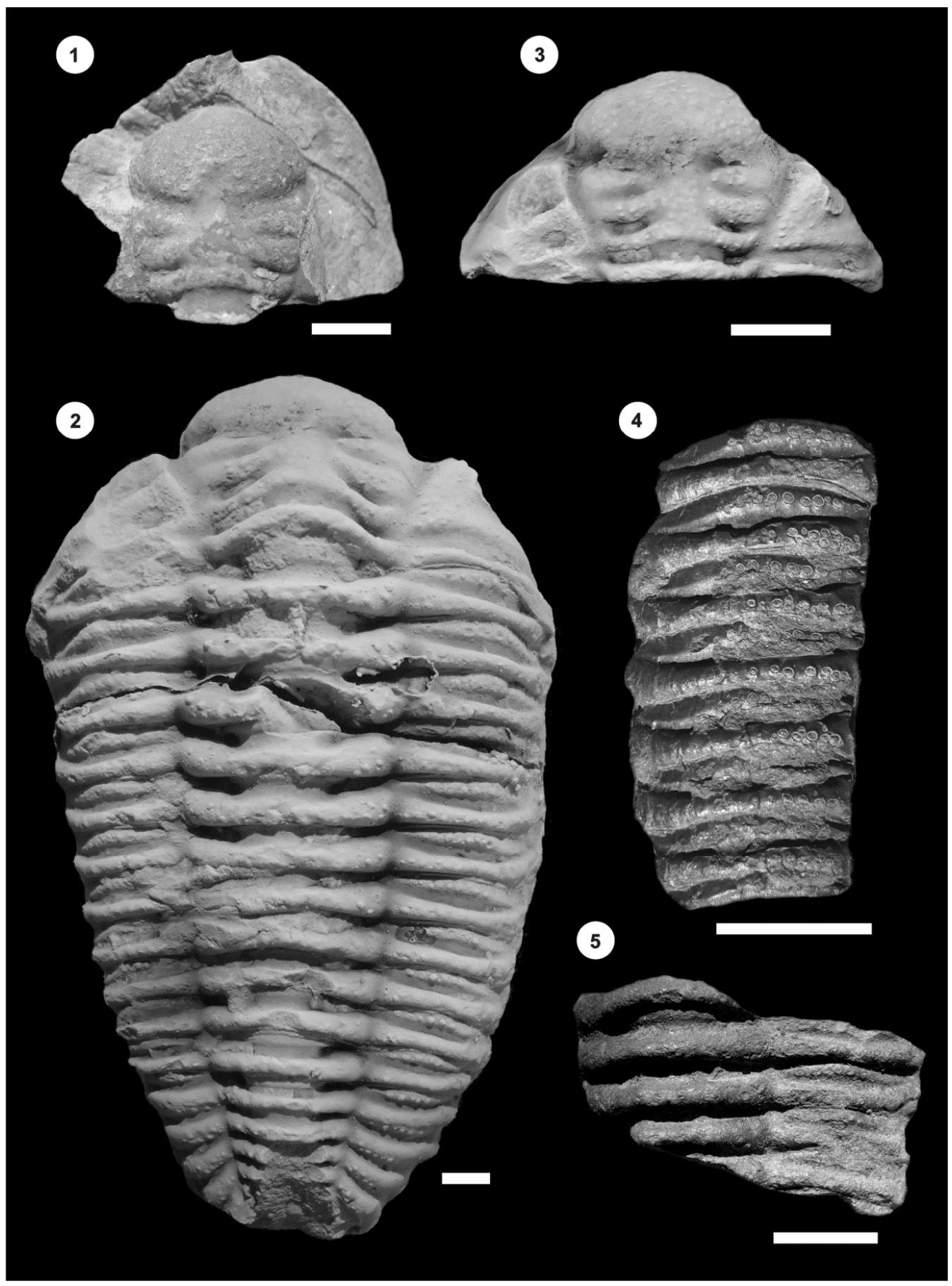

Figure 2. 1-5, Metacryphaeus tuberculatus. Specimens collected from $2 \mathrm{~km}$ south of Picos (1) and from São João Vermelho (2-5). 1, cephalon in dorsal view, AMNH 063283; 2, 3, articulated specimen with cephalon, thorax and pygidium and detail of cephalon in dorsal view, FUMDHAM 197602; 4, Fragment of thoracic pleurae in dorsal view, FUMDHAM collection (no identification number); 5, Thoracic segments in dorsal view, FUMDHAM collection (no identification number). Scale bar $=1 \mathrm{~cm}$. 
Metacryphaeus meloi Carvalho et al. (1997)

Figures 3.1-5

1968. Metacryphaeus australis Castro, p. 485, pl. 3, figs. $2-5$.

1987. Metacryphaeus cf. australis Caldas et al., p. 539, phot. 01-03.

1994. Metacryphaeus meloi Carvalho et al., p. 119 (nomen nudum).

1997. Metacryphaeus meloi Carvalho et al., p. 6-9, fig. 3 A-G.

?2013. Metacryphaeus cf. australis Leme et al., p. 19, fig. 3G.

Emended diagnosis. Cephalon subtriangular, with median frontal dense glabella short process; glabella densely covered with small, relatively subdued tubercles; genal angles bearing a triangular spine, not parallel to the lateral border, standing outward the gena; eyes distinctly separated from axial furrows, moderately oblique (exsag.). Pygidial pleurae terminate as blunt spines; axial rings convex forward medially.

Examined material. MCT-DNPM (5009, 5012, 6153, 6155); FUMDHAM 169046; AMNH 63283; MPEG (1270-I, 1271-I, 1273-I, 1275-1302-I); IG-UFRJ (37-39-Tr, 41-48-Tr, 55-58$\operatorname{Tr})$.

Geographic and stratigraphic provenance. Early Givetian of Passagem Member, Pimenteira Formation in the vicinities of Oiti village, Pimenteiras city, eastern Piauí State (type locality of the Passagem Member); also in the km 305 of road BR 316, close to Picos city and near Barreiro Branco village, Sussuapara city, eastern Piauí State; and possibly from late Eifelian-early Givetian of Pimenteira Formation in São João Vermelho village, João Costa city, southeastern Piauí State, Brazil.

Discussion. Metacryphaeus meloi is inserted in the "Metacryphaeus tuberculatus group" (Lieberman, 1993) due to the presence of exoskeletal tuberculation; triangular anterior median process, which extends beyond the glabella; rounded glabellar frontal lobe (LA) and anterior edge of the eyes displaced from the axial furrow (Carvalho et al., 1997). It is the closest relative of M. tuberculatus, sharing most of its characters with this species. Nevertheless, M. meloi is differentiated by its less inflated frontal frontal glabellar lobe, narrower cephalic axial furrows, less conspicuous tubercles on the cephalon and less defined lateral glabellar lobes (Carvalho et al., 1997). Carvalho et al. (1997) also pointed the pygidial axial rings vaulted forward medially 
in M. meloi, albeit this feature is also present in a pygidium illustrated in Braniša (1965: pl. 21, fig. 14), attributed to M. tuberculatus by Lieberman (1993, p. 562).

The presence of genal spines in some specimens, including a paratype housed at the Instituto de Geociências of the Universidade Federal do Rio de Janeiro (IG-UFRJ) (Figs.3.1-4) adds a previously undescribed feature to the diagnosis of $M$. meloi. The genal spine is triangular, not parallel to the lateral border, standing outward the gena.

The morphology of the genal spine is quite similar to that illustrated in Metacryphaeus caffer Salter, 1856 by Cooper (1982: fig. 61).

Carvalho et al. (1997) observed variations in the convexity of frontal lobe (sag.) and in the depth of the posterior median impression (PMI) incision, attributing these features to intraspecific variation. The wider genae in two specimens, with length of glabella / width of cephalon ratios of about $42 \%$ and $48 \%$ (Fig.3.5), is suggested here as an additional intraspecific variation of $M$. meloi. These values differ from the 55\% ratio observed by Carvalho et al. (1997).

The specimen FUMDHAM 169046 (Fig.3.6) from the Pimenteira Formation at João Costa is here tentatively ascribed to $M$. meloi, based on the shape of terminal lappet, posterior pleural rib tips curved backwards and the absence of tubercles. This find may indicate a new occurrence for this taxon, currently known from the eastern area of Piauí State in the region of Picos city and the villages of Oiti and Barreiro Branco. However, additional specimens in better preservational conditions are required to confirm this occurrence. 


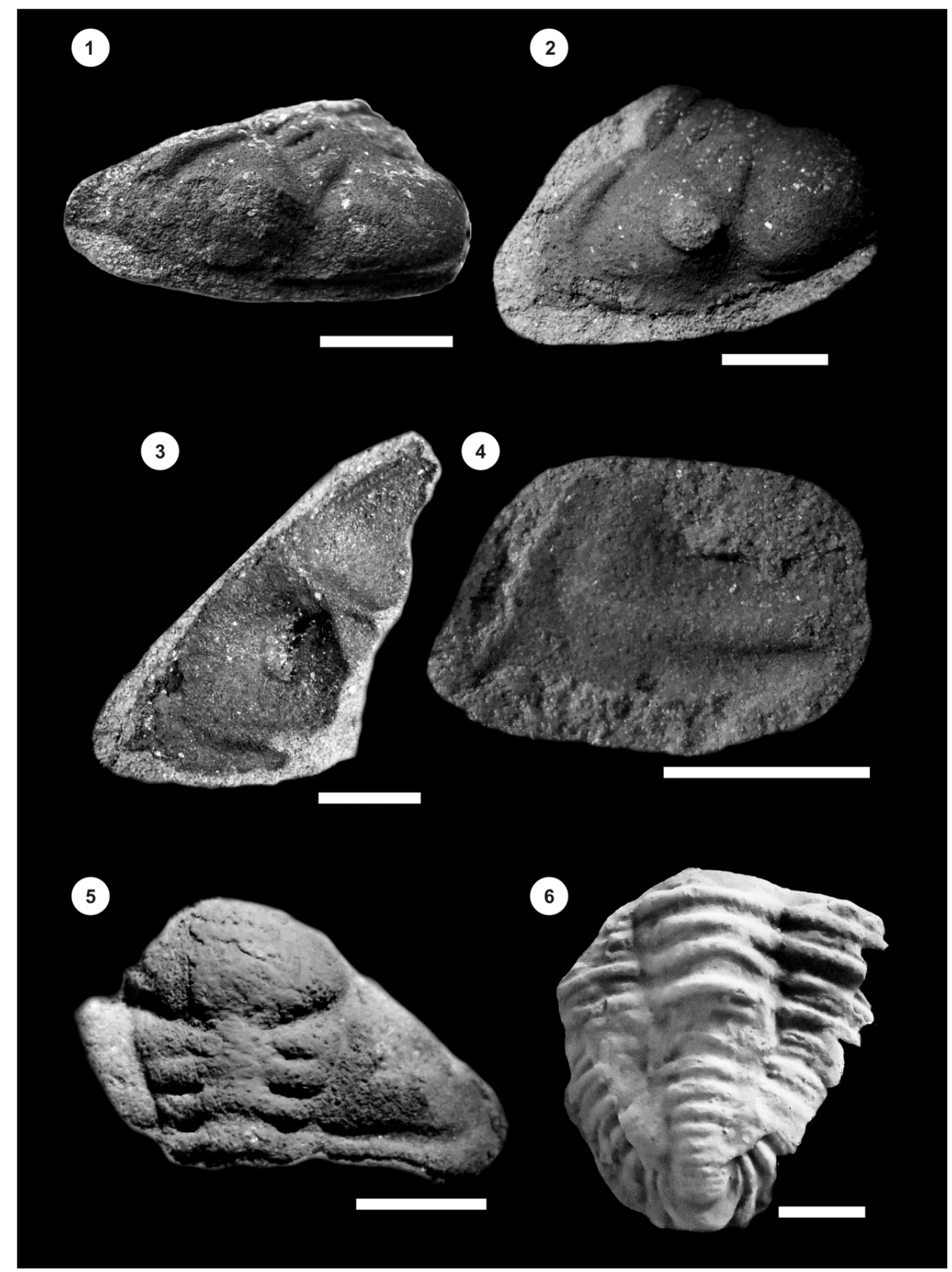

Figure 3. 1-5, Metacryphaeus meloi. Specimens collected from km 305 of BR 316 in Picos (14) and from Barreiro Branco (5). 1, Cephalon in oblique view, IG-UFRJ 48-Tr; 2, cephalon in lateral view,MPEG 1271-I; 3, Cephalon fragment in ventral view, MPEG 1271-I (external mold of 3.2); 4, Gena fragment in ventral view, MPEG 1271-I; 5, cephalon in dorsal view, MPEG 1297-I; 6, Metacryphaeus cf. meloi. Specimen collected from São João Vermelho. Thoracopygidium in dorsal view, FUMDHAM 169046. Scale bar $=1 \mathrm{~cm}$. 


\section{METACRYPHAEUS TUBERCULATUS FROM PIMENTEIRA FORMATION}

The presence of Metacryphaeus tuberculatus in late Eifelian-early Givetian Pimenteira Formation of the Parnaíba Basin claimed by Lieberman et al. (1991) and Lieberman (1993) are substantiated by the discovery of samples here attributed to this species from São João Vermelho village, in João Costa city, southeastern Piauí State, which includes an articulated specimen (FUMDHAM 197602, Figs. 2.2-3), with tubercles on the exoskeleton and broad, well divergent axial furrows.

The Parnaíba Basin yields the greatest diversity of Metacryphaeus species among Brazilian basins, most of which found in the Pimenteira Formation; in addition to M. tuberculatus and $M$. meloi, this unit also includes Metacryphaeus kegeli from the region of Pimenteiras (Kegel, 1953; Carvalho et al., 1997) and Picos (Ponciano et al., 2012) cities, eastern Piauí State. This species, together with M. meloi, are exclusive of the Parnaíba Basin. Besides these Pimenteira Formation occurrences, rare samples attributed to Metacryphaeus sp. were found at the base of Longá Formation, of Famennian age (Carvalho, 1995).

In an inventory of the paleontologically important sites of the Pimenteira Formation in the Piauí State, Ponciano et al. (2012) included São João Vermelho among the most relevant areas for paleontological studies. The presence of Metacryphaeus tuberculatus in this locality increases its importance for future field works. Besides, if confirmed that M. meloi is present in deposits of São João Vermelho, this will indicate a new occurrence for this species, suggesting an overlapped geographic range between it and M. tuberculatus. Also, it would record a different preservation mode for $M$. meloi, concretion in finer sediments, differing from the molds preserved in sandier sediments typical of the Passagem Member.

The Metacryphaeus tuberculatus in the Parnaíba Basin suggests a connection between this basin and Bolivia, where the taxon is present in Early to Middle Devonian strata (Wolfart, 1968; Lieberman, 1993). This connection may not have happened prior to the Eifelian, as chitinozoans typical to the Paraná Basin, the likely seaway between Bolivia and Parnaíba, were not present in the latter locality during the Early Devonian (Lange, 1967). 


\section{THE "METACRYPHAEUS TUBERCULATUS GROUP"}

Members of the "Metacryphaeus tuberculatus group" (Lieberman, 1993) include Metacryphaeus tuberculatus from Bolívia and Parnaíba Basin, M. meloi from the Parnaíba Basin, M. australis from the Paraná Basin, Metacryphaeus allardyceae (Clarke, 1913) from West Falkland Island and M. caffer from South Africa and possibly Falkland Islands (Carvalho, 2006). All the representatives of this group were extant contemporaneously except for $M$. allardyceae that appeared and vanished before the other tuberculatus occurrences (Lieberman, 1993). They possess great similarity between each other, with the differences between them being subtle (Cooper, 1982; Edgecombe, 1994). Criteria usually used to differentiate the species of this group are the position of the eyes relative to the axial furrows, the shape and extension of pygidial marginal lappets (Lieberman et al., 1991; Edgecombe, 1994) and the the size of the eye, measured through the Large Eye Index (Lieberman et al., 1991). In this concern, M. australis from the Paraná Basin is readily differentiated from $M$. tuberculatus by its larger eyes immediately adjacent to axial furrows, and its longer and blade-like pygidial lappets (Lieberman et al., 1991). Contrarily, there have been some controversies on the differentiation between $M$. tuberculatus, M. caffer and M. allardyceae. Cooper (1982) considers that M. tuberculatus and M. caffer are probably conspecific, based on the great similarity between the cephala. Lieberman et al. (1991) kept M. tuberculatus and M. caffer as separated taxa, yet admitted that differences between them are slight. Edgecombe (1994) noted that $M$. allardyceae is very similar to $M$. tuberculatus, differing only in the antero-median process more triangular in M. allardyceae.

\section{ACKNOWLEGDMENTS}

F. van E. Meira and F. A. Carbonaro thank the Fundação de Amparo à Pesquisa do Estado de São Paulo (FAPESP) for financial support (Process numbers 2012/07075-3 and 2013/09683-3 respectively). The authors thank Niède Guidon (FUMDHAM) for lending the samples from João Costa area illustrated herein. Bushra Hussaini (AMNH), Ismar de Souza Carvalho, Flávia Alessandra Figueiredo, Mônica de Medina Coeli (IG-UFRJ), Maria Inês Ramos, Heloisa Maria Moraes Santos, Ana Paula Linhares Pereira (MPEG), Rodrigo da Rocha Machado, Irma Tie Yamamoto and Rita de Cassia Tardin Cassab (MCT-DNPM) are thanked for permitting the access to the collections and for helping with the location of exemplars. F. van E. Meira thanks 
Angela Meira Faleiros for article revision and further discussions, and Andrea Soledad Herrera Faleiros for revising the abstract in Spanish.

\section{REFERENCES}

Almeida, F.F.M., Hasui, Y., Brito Neves, B.B., and Fuck, R.A. 1977. Províncias estruturais brasileiras. $8^{\circ}$ Simpósio de Geologia do Nordeste (Campina Grande), Proceedings: 363-391.

Beurlen, K. 1965. Observações no Devoniano do Estado do Piauí. Anais da Academia Brasileira de Ciências 37: 61-67.

Braniša, L. 1965. Los fossiles guias de Bolivia. I. Paleozoico. Servicio Geologico de Bolivia, Boletin 6: 1-282.

Breuer, P., and Grahn, Y. 2011. Middle Devonian spore stratigraphy in the eastern outcrop belt of the Parnaíba Basin, northeastern Brazil. Revista Española de Micropaleontología 43: $19-38$.

Caldas, E.B., Florêncio, C.P. and Lima Filho, F.P. 1987. Nova ocorrência de Metcryphaeus cf. australis no Devoniano Médio da Bacia do Parnaíba. $10^{\circ}$ Congresso Brasileiro de Paleontologia (Rio de Janeiro), Proceedings: 539- 544.

Caputo, M.V., Iannuzzi, R., and Fonseca, V.M.M. 2005. Bacias sedimentares brasileiras: Bacia do Parnaíba. Phoenix 81: 1- 6.

Carvalho, M.G.P. 1995. [Trilobitas devonianos da Bacia do Parnaíba (formações Pimenteira, Cabeças e Longá). PhD Thesis, Programa de Pós-graduação em Geologia, Universidade Federal do Rio de Janeiro, Rio de Janeiro, 132 p., Unpublished].

Carvalho, M.G.P. 1999. Remarks on the calmoniid trilobites from the Middle Devoniano f the Parnaíba Basin, NE Brazil. $16^{\circ}$ Congresso Brasileiro de Paleontologia (Crato), Abstracts: 34.

Carvalho, M.G.P. 2006. Devonian trilobites from the Falkland Islands. Palaeontology 49 2134.

Carvalho, M.G.P., Edgecombe, G.D., and Lieberman, B.S. 1994. Devonian calmoniid trilobites from the Parnaíba Basin, Piauí State, Brazil. Anais da Academia Brasileira de Ciências 66 (1): 119 . 
Carvalho, M.G.P., Edgecombe, G.D., and Lieberman, B.S. 1997. Devonian calmoniid trilobites from the Parnaíba Basin, Piauí State, Brazil. American Museum Novitates 3192: 1- 11.

Castro, J.S. 1968. Trilobitas da Formação Pimenteiras, Devoniano do estado do Piauí. Anais da Academia Brasileira de Ciências 40: 481- 489.

Clarke, J. M. 1913. Fósseis devonianos do Paraná. Monografia do Serviço Geológico e Mineralógico do Brasil 1: 1-353.

Cooper, M.R. 1982. A revision of the Devonian (Emsian - Eifelian) trilobita from the Bokkeveld Group of South Africa. Annals of the South African Museum 89: 1- 174.

Della Fávera, J.C. 1990.[Tempestitos da Bacia do Parnaíba. Um ensaio holístico. PhD Thesis, Programa de Pós-graduação em Geociências, Universidade Federal do Rio Grande do Sul, Porto Alegre, 243 p., Unpublished].

Delo, D.M. 1935. A revision of the phacopid trilobites. Journal of Paleontology 9: 402-420.

Edgecombe, G.D. 1994. Calmoniid trilobites from the Devonian Fox Bay Formation, Falkland Islands. New York State Museum Bulletin 481: 55- 68.

Eldredge, N., and Braniša, L. 1980. Calmoniid trilobites of the Lower Devonian Scaphiocoelia Zone of Bolivia, with remarks on related species. Bulletin of the American Museum of Natural History 165:181- 289.

Fonseca, V.M.M., and Melo, J.H.G. 1987. Ocorrência de Tropidoleptus carinatus (Conrad) (Brachiopoda, Orthida) na Formação Pimenteira, e sua Importância Paleobiogeográfica. $10^{\circ}$ Congresso Brasileiro de Paleontologia (Rio de Janeiro), Proceedings: 505- 537.

Fonseca, V.M.M., and Ponciano, L.C.M.O. 2011. Braquiópodes do Devoniano Médio das bacias do Amazonas e Parnaíba. In: I.S. Carvalho, N.K. Srivastava, O. Strohschoen Jr., and C.C. Lana (Orgs.). Paleontologia: cenários de vida. Interciência, Rio de Janeiro, p. 169-190.

Góes, A.M.O. 1995. [A Formação Poti (Carbonífero Inferior) da Bacia do Parnaíba. PhD Thesis, Programa de Pós-Graduação em Geologia Sedimentar, Universidade de São Paulo, São Paulo, 171 p., Unpublished].

Grahn, Y., Melo, J.H.G., and Loboziak, S. 2006. Integrated Middle and Late Devonian miospore and chitinozoan zonation of the Parnaíba Basin, Brazil: an update. Revista Brasileira de Paleontologia 9: 283-294. 
Grahn, Y., Young, C., and Borghi, L. 2008. Middle Devonian chitinozoan biostratigraphy and sedimentology in the eastern outcrop belt of the Parnaíba Basin, northeastern Brazil. Revista Brasileira de Paleontologia 11: 137-146.

Harrington, H.J., Henningsmoen, G., Howell, B.F., Jaanusson, V., Lochman-Balk, C., Moore, R.C., Poulsen, C., Rasetti, F., Richter, E., Schmidt, H., Sdzuy, K., Struve, W., Størmer, L., Stubblefield, C.J., Tripp, R., Weller, J.M., and Whittington, H.B.1959. Trilobita. In: R.C. Moore (Ed.), Treatise on Invertebrate Paleontology Part O. Arthropoda, Geological Society of America and University of Kansas Press, p. O38 - O526.

Kegel, W. 1953. Contribuição para o estudo do Devoniano da Bacia do Parnaíba. Boletim da Divisão de Geologia e Mineralogia 141: 1- 48.

Kozlowski, R. 1923. Faune Dévonienne de Bolivie. Annales de Paleontologie 12: 1- 112.

Lange, F.W. 1967. Biostratigraphic subdivision and correlation of the Devonian in the Paraná Basin. In: J.J. Bigarella (Ed.), Problems in Devonian Brazilian geology. Boletim Paranaense de Geociências, Curitiba, 21/22: 63-98.

Leme, J.M., Meira, F.van E., Stasi, A.M.D., and Soares, S.P. 2013. The occurrence of Phacopida trilobites from Pimenteira Formation at João Costa, Piauí, Brazil. Geologia USP - Série Científica 13: 17-22.

Lieberman, B. S. 1993. Systematics and biogeography of the "Metacryphaeus Group" Calmoniidae (Trilobita, Devonian), with comments on adaptative radiations and the geological history of the Malvinokaffric Realm. Journal of Paleontology 67: 549-570.

Lieberman, B. S., Edgecombe, G. D., and Eldredge, N. 1991, Systematics and biogeography of the "Malvinella Group", Calmoniidae (Trilobita,Devonian). Journal of Paleontology 65: $824-843$.

Melo, J.H.G., 1985. [A província Malvinocáfrica no Devoniano do Brasil: estado atual dos conhecimentos. Master's Dissertation, Programa de Pós-graduação em Geologia, Universidade Federal do Rio de Janeiro, Rio de Janeiro, 890 p., Unpublished].

Melo, J.H.G., 1988. The Malvinokaffric realm in the Devonian of Brazil. In: N.J. McMillan, A.F. Embry, and O.J. Glass (Eds.). Devonian of the world. Canadian Society of Petroleum Geologists, Proceedings of the $2^{\text {nd }}$ International Symposium on the Devonian System, Calgary, C.S.P.G., 1987 Memoir, 14 (1): 667- 703. 
Plummer, F. 1948. Estados do Maranhão e Piauí. In: BRASIL, Conselho Nacional do Petróleo. Relatório de 1946: 87-134.

Ponciano, L.C.M.O., Fonseca, V.M.M., and Machado, D.M.C. 2012a. Taphofacies analysis of late early Givetian fossil assemblages of the Parnaíba Basin (State of Piauí, northeast Brazil). Palaeogeography, Palaeoclimatology, Palaeoecology 326: 95-108.

Ponciano, L.C.M.O., Castro, A.R.S.F., Fonseca, V.M.M., and Machado, D.M.C. 2012b. Tafocenoses da Formação Pimenteira, Devoniano da Bacia do Parnaíba, Piauí: mapeamento, inventário e relevância patrimonial. Anuário do Instituto de Geociências UFRJ 35: 5- 27.

Reed, F.R.C. 1907. The fauna of the Bokkeveld Beds. Geological Magazine 4: 222-232.

Salter, J.W. 1856. Description of Palaeozoic Crustacea and Radiata from South Africa. Transactions of the Geological Society of London 7: 215-224.

Salter, J. W. 1864. British fossils. Memoirs Geological Survey of Great Britain, Decade 11.

Soares, S.P., Simões, M.G., and Leme, J.M. 2008. Metacryphaeus rotundatus, um novo elemento da fauna de trilobites Calmoniidae (Phacopida) da Formação Ponta Grossa, (Devoniano), Bacia do Paraná, Brasil. Geologia USP - Série Científica 8 (1): 15- 24.

Vaz, P.T., Rezende, N.G.A.M., Wanderley Filho, J.R., and Travassos, W.A.S. 2007. Bacia do Parnaíba. Boletim de Geociências da Petrobras 15: 253- 263.

Whittingon, H.B., Chatterton, B.D.E., Speyer, S.E., Fortey, R.A., Owens, R.M., Chang, W.T., Dean, W.T., Jell, P.A, Lawrie, J., Palmer, A.R., Repina, L.N., Rushton, A.W.A., Shergold, J.H., Clarkson, E.N.K., Wilmot, N.V., and Kelly, S.R.A. 1997. Treatise on Invertebrate Paleontology, Pt. O, Arthropoda 1, Trilobita (revised edition). Geological Society of America and the University of Kansas Press, Lawrence, 530 p.

Wolfart, R. 1968. Die Trilobiten aus dem Devon Boliviens und ihre Bedeutung für Stratigraphie und Tiergeographie, 5-201. In: R. Wolfart, and A. Voges. Beiträge zur Kenntnis des Devons von Bolivien. Beihefte zum Geologischen Jahrbuch 74. 


\begin{abstract}
ANEXO III
METACRYPHAEUS TUBERCULATUS (KOZLOWSKI, 1923) AND M. AUSTRALIS (CLARKE, 1913) (TRILOBITA, PHACOPIDA) FROM THE DEVONIAN OF PARANÁ BASIN: TAXONOMY AND PALAEOBIOGEOGRAPHY
\end{abstract}

Artigo Submetido ao Periódico Ameghiniana 


\section{Enc: [AMGH] Submission Acknowledgement}

2 messages

Fri, May 20, 2016 at 8:12 AM

\section{Fábio Augusto Carbonaro}

Doutorando do Programa de Pós-Graduação em Biologia Comparada - FFCLRP - USP - Ribeirão Preto (SP)

Em Segunda-feira, 16 de Novembro de 2015 16:52, Comité Editor <comiteeditor@ameghiniana.org.ar> escreveu:

Fábio Augusto Carbonaro:

Thank you for submitting the manuscript, "Metacryphaeus tuberculatus (Kozlowski, 1923) and M. australis (Clarke, 1913) (Trilobita, Phacopida) from the Devonian of Paraná Basin: taxonomy and palaeobiogeography" to Ameghiniana. With the online journal management system that we are using, you will be able to track its progress through the editorial process by logging in to the journal web site:

Manuscript URL:

http://www.ameghiniana.org.ar/index.php/ameghiniana/author/submission/2966

Username: fabiocarbonaro

If you have any questions, please contact me. Thank you for considering this journal as a venue for your work.

Comité Editor

Ameghiniana

\section{Ameghiniana \\ http://www.ameghiniana.org.ar/index.php/ameghiniana}


METACRYPHAEUS TUBERCULATUS (KOZLOWSKI, 1923) AND M. AUSTRALIS (CLARKE, 1913) (TRILOBITA, PHACOPIDA) FROM THE DEVONIAN OF PARANÁ BASIN: TAXONOMY AND PALAEOBIOGEOGRAPHY

F. B. Carbonaro ${ }^{1}$, F. van. E. Meira ${ }^{2}$, J. de M. Leme ${ }^{3}$, E.P. Bosetti ${ }^{4}$, R. P. Ghilardi ${ }^{5}$

${ }^{1}$ Programa de Pós-graduação em Biologia Comparada, Faculdade de Filosofia, Ciências e Letras de Ribeirão Preto, Universidade de São Paulo, 14040-901 Ribeirão Preto, São Paulo, Brazil.

${ }^{2}$ Programa de Pós-graduação em Geoquímica e Geotectônica, Instituto de Geociências, Universidade de São Paulo, 05508-080 São Paulo, São Paulo, Brazil.

${ }^{3}$ Departamento de Geologia Sedimentar e Ambiental, Instituto de Geociências, Universidade de São Paulo, 05508-080 São Paulo, São Paulo, Brazil.

${ }^{4}$ Universidade Estadual de Ponta Grossa, Laboratório de Estratigrafia e Paleontologia, Departamento de Geociências, UEPG, 84010-919 Ponta Grossa, Paraná, Brazil.

${ }^{5}$ Universidade Estadual Paulista Júlio de Mesquita Filho, Faculdade de Ciências de Bauru, Departamento de Ciências Biológicas, UNESP Bauru, 17033-360 Bauru, São Paulo, Brazil.

*Corresponding author:fabiocarbonaro@yahoo.com.br 
Abstract. The calmoniid trilobites of the genus Metacryphaeus include M. tuberculatus, M. kegeli, M. meloi, M. rotundatus, M. giganteus, M. convexus, M. curvigena, M. branisai, M. caffer, M. australis, and M. allardyceae. The geographic distribution of this genus includes many areas of Gondwana as Bolivia, Peru, Brazil, the Falkland Islands, and South Africa. This study reports a new occurrence of $M$. tuberculatus in the Paraná Basin (Goiás and Paraná states, Brazil) both in the Alto Garças and Apucarana sub-basins. M. tuberculatus was compared with all Metacryphaeus species, especially $M$. australis which also presents a new record in the Paraná Basin. These new records of $M$. tuberculatus imply a different dispersion interpretation of that known in the literature. M. tuberculatus seems to have originated during the Early Devonian and lived in areas as Bolivia, Peru, and Apucarana Sub-basin (Paraná Basin, Brasil). Probably the dispersion between these areas is related to the Emsian transgression. During the Givetian, the species probably migrates to Alto Garças Sub-basin (Paraná Basin, Brasil) and Parnaíba Basin where it has records. These facts provide a different paleogeographic interpretation compared to that presented by Tropidoleptus and Exaesiodiscus in the Gondwana and suggest a different pattern of migration for the Devonian of Brazil.

Key words. Alto Garças Sub-basin, Apucarana Sub-basin, Brazil, dispersion

Resumen. Los trilobites calmoniid del género Metacryphaeus incluyen M. tuberculatus, M. kegeli, M. meloi, M. rotundatus, M. giganteus, M. convexus, M. curvigena, M. branisai, M. caffer, M. australis y M. allardyceae. La distribución geográfica de este género incluye muchas áreas de Gondwana como Bolivia, Perú, Brasil, las Islas Malvinas y Sudáfrica. Este estudio reporta una nueva ocurrencia de $M$. tuberculatus en la Cuenca del Paraná (estados de Goiás y Paraná, Brasil), tanto en las sub-cuencas Alto Garças y Apucarana. M. tuberculatus fue comparado con todas las especies de Metacryphaeus, especialmente M. australis, que también presenta un nuevo registro en la Cuenca del Paraná. Estos nuevos registros de M. tuberculatus implican una interpretación de dispersión diferente de la que se conoce en la literatura. M. tuberculatus parece tener su origen durante el Devónico Temprano en zonas como Bolivia, Perú y Apucarana Sub-cuenca (Cuenca del Paraná, Brasil). Probablemente la dispersión entre estas áreas está relacionada con la transgresión Emsiense. Durante el Givetiense, la especie probablemente emigra a Alto Garças Sub-cuenca (Cuenca del Paraná, Brasil) y la cuenca del Parnaíba, donde tiene registros. Estos hechos proporcionan una interpretación paleogeográfica 
diferente en comparación con la presentada por Tropidoleptus y Exaesiodiscus en el Gondwana y sugieren un patrón diferente de la migración para el Devónico de Brasil.

Palavras clave. Alto Garças Sub-cuenca, Apucarana Sub-cuenca, Brasil, dispersión 


\section{INTRODUCTION}

TRILOBITES are conspicuous elements of the macrofauna of marine invertebrates from the early Cambrian to the late Permian. During their 250 million of years of existence, the trilobites have inhabited several types of marine environments, both shallow and deep, and have adopted different lifestyles accordingly (e.g. predators/scavengers, grazers, filter feeders and particle feeders) (Fortey, 2014).

During the Devonian, some trilobite species were present in the endemic "Austral Fauna," (Clarke, 1913) now referred to as the Malvinokaffric Realm (Richter, 1941; Richter and Richter, 1942; Boucot, 1971, 1974, 1985; Eldredge and Ormiston, 1979). Among the trilobite families contained in this fauna, the Calmoniidae, made up of several genera, were prominent (e.g. Calmonia Clarke, 1913, Typhloniscus Salter, 1856, Plesioconvexa Lieberman, 1993, Punillaspis Baldis and Longobucco, 1977, Eldredgeia Lieberman, 1993, Clarkeaspis Lieberman, 1993, Malvinocooperella Lieberman, 1993, Wolfartaspis Cooper, 1982). Our study focuses on the trilobites of the calmoniid genus Metacryphaeus Reed, 1907.

Metacryphaeus occurs in Devonian rocks from the Praguian to the Eofrasnian in Brazil, Bolivia, Peru, the Falkland Islands, and South Africa (Clarke, 1913; Cooper, 1982; Lieberman, 1993; Carvalho et al., 1997; Ghilardi and Simões, 2007; Velazco, 2012), and in Brazil and Bolivia, it is present in more than one geological unit (Paraná and Parnaíba basins in Brazil; Belén, Sicasica, Icla, and Limoncito formations in Bolivia; Clarke, 1913; Lieberman, 1993; Carvalho et al., 1997). The species represented in the Gondwana include M. tuberculatus (Kozlowski, 1923), M. kegeli Carvalho et al., 1997, M. meloi Carvalho et al., 1997, M. rotundatus (Kozlowski, 1923), M. giganteus (Ulrich, 1892), M. convexus (Ulrich, 1892), M. curvigena Lieberman, 1993, M. branisai Lieberman, 1993, M. caffer (Salter, 1856), M. australis (Clarke, 1913), and M. allardyceae (Clarke, 1913).

According to Lieberman et al. (1991), intraspecific variations are quite common in $M$. tuberculatus, M. caffer, and M. australis and can cause problems to identify these organisms. However, for these authors some characters show better the differences between these species as the kind of tuberculation, the Large Eye Index (LEI), and the position of the anterior margin of the eyes in relation to the axial furrow and the glabellar furrow S2. 
In view of these problems, we show that M. tuberculatus, which has been recorded from the Devonian of Bolivia and the Parnaíba Basin of Brazil, also occurs in the Paraná Basin of Brazil in both the Alto Garças (Chapada Group Unit 4) and Apucarana (Ponta Grossa Formation) subbasins (Figs. 1 and 2). These new occurrences of M. tuberculatus in the Paraná Basin were systematically classified and compared with all species of Metacryphaeus found in the Devonian of Gondwana (M. giganteus, M. convexus, M. curvigena, M. branisai, M. kegeli, M. meloi, M. rotundatus, $M$. caffer, $M$. australis, and $M$. allardyceae), mainly with the new records of $M$. australis in the Paraná Basin.

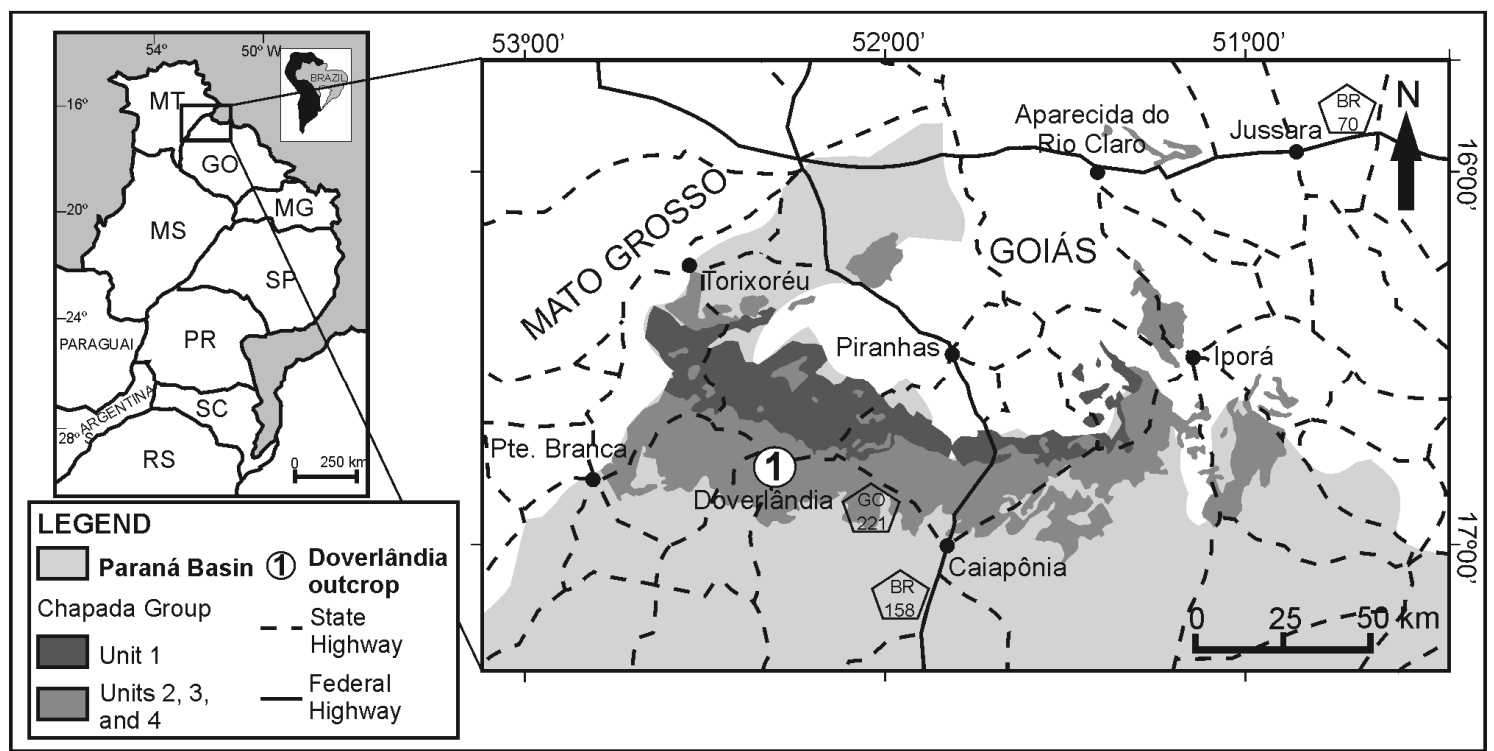

Figure 1. Localization map of the Doverlândia outcrop (Goiás State, Brazil) where $M$. tuberculatus was collected (Alto Garças Sub-basin, Paraná Basin).

The presence of M. tuberculatus in the Paraná Basin allowed a discussion about the dispersion of this species during the Devonian which was associated to the transgressive-regressive cycles, quite common in this period (Assine et al., 1998; Assine, 2001; Grahn et al., 2010b; Bosetti et al., 2012). Eustatic high level episodes were better evidenced in the Emsian and Givetian (Assine et $a l ., 1998)$ and is very probable its relation to the dispersion of the genus Metacryphaeus through marine connections between the basins during the Devonian.

Some organisms are indicators of these connections between the brazilian palaeozoic basins during the Devonian as brachiopods and crinoids of the genus Tropidoleptus and Exaesiodiscus 
(Fonseca and Melo, 1987; Scheffler, 2010; Scheffler et al., 2011). However, M. tuberculatus is a new species that confirm these connections.

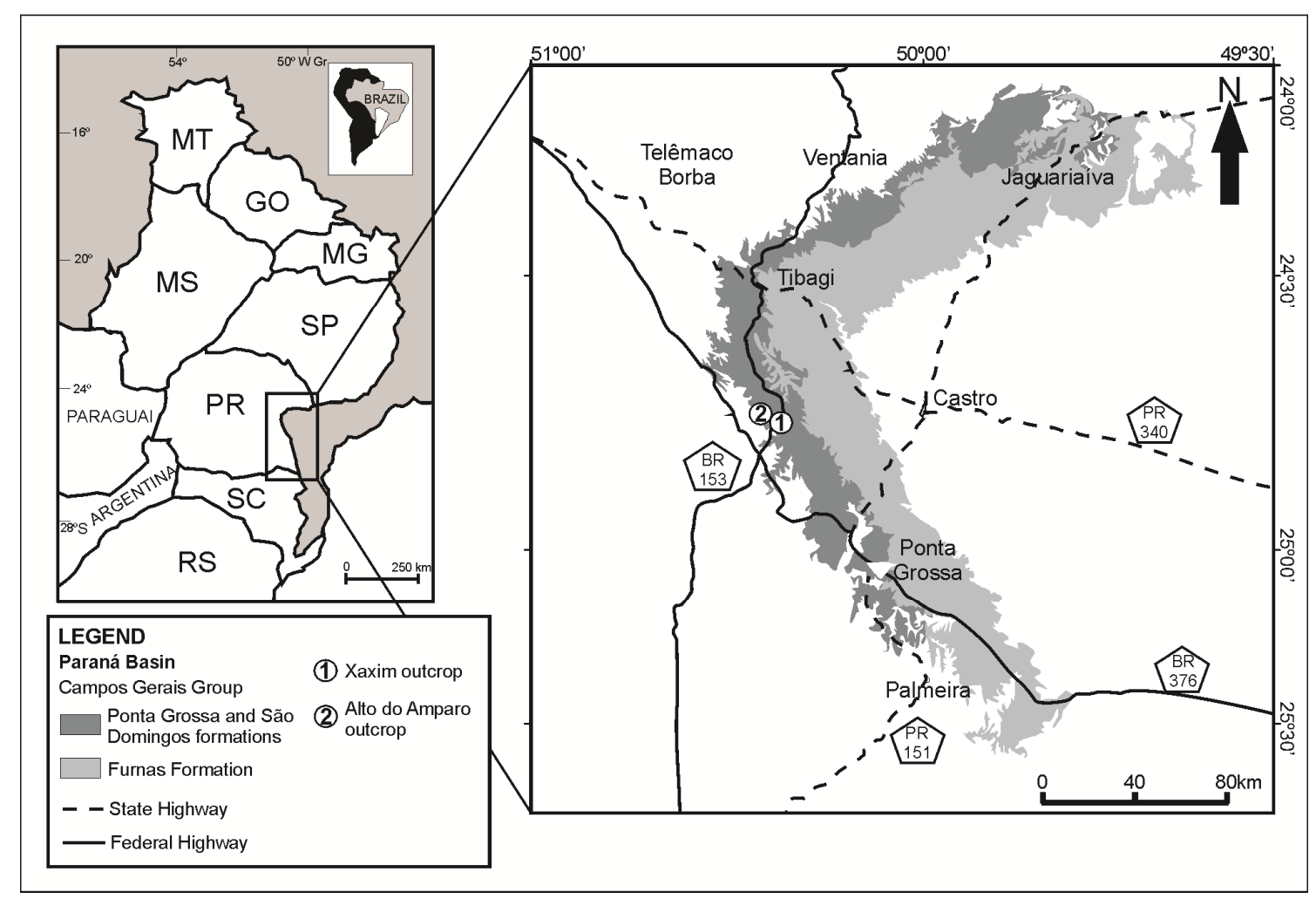

Figure 5.2: Localization map of Xaxim and Amparo outcrops, where M. australis occur (Apucarana Sub-basin, Paraná Basin, Brazil).

In sight of what was exposed here, the present work presents taxonomic and palaeobiogeographic interpretations about the new occurrence of M. tuberculatus in the Paraná Basin making a comparison with $M$. australis. 


\section{GEOLOGICAL SETTING}

The Paraná Basin covers an area of approximately $1.100 .000 \mathrm{~km}^{2}$ of the Brazilian territory comprising part of the states of Rio Grande do Sul, Santa Catarina (only subsurface), Paraná, São Paulo, Goiás, Minas Gerais, Mato Grosso, and Mato Grosso do Sul (Petri and Fulfaro, 1988; Melo, 1988; Milani et al., 2007). During the Devonian, the exposition area of this basin was marked by a division which was identified through sedimentological studies and subsurface stratigraphic dating. Thus, two sub-basins were deposited during this period: the Alto Garças (in the north) and the Apucarana (in the south) which was separated by Três Lagoas and Campo Grande archs, also originated in the Devonian (Ramos, 1970). According to Melo (1988), the marine connection between these sub-basins just happened during the Givetian transgression.

Although the depositional environment of both sub-basins has been marine, they evolved differently, and the deposition that occurred in the Alto Garças Sub-basin had more characteristics of shallow environments as large amount of siltstones and sandstones. Due to this, it is difficult to use the same geological units of Apucarana Sub-basin (Furnas, Ponta Grossa, and São Domingos formations) for Alto Garças Sub-basin which becomes more suitable to use the Chapada Group Units 1, 2, 3, and 4 for Alto Garças Sub-basin (Melo, 1988; Grahn et al., 2010a).

The litology of the Unit 1 is similar to Furnas Formation of Apucarana Sub-basin and comprises fine and coarse sandstones (Melo, 1988). According to Grahn et al. (2010a), this unit probably presents the Lochkovian age.

The Unit 2 presents a basal conglomerate covered by fine purple-reddish sandstone with sitstones and shales interbeds. The top of this unit consists in fine-middle red-grayish sandstones. These rocks are bioturbated (Andrade and Camarço, 1980). This unit was deposited in two intervals which were separated by a depositional gap. The lower interval has the Neopraguian-Eoemsian age and the upper interval has the Neoemsian-Eifelian age (Grahn et al., 2010a). The lower portion of Unit 2 is chronocorrelate to the Ponta Grossa Formation (Apucarana Sub-basin) which presents sandstones interbedded by siltstones and followed by sandy shales (Grahn et al., 2010b).

The litologic composition of Unit 3 comprises middle-coarse reddish sandstones with conglomeratic levels, characteristics of deltaic deposition in shallow marine environments 
heavily influenced by waves (Andrade and Camarço, 1980; Grahn et al., 2010a). The transitional beds between the upper portion of Unit 2 and Unit 3 have the same fauna found in the Tibagi Member of São Domingos Formation (Apucarana Sub-basin) which suggest the Neoemsian age for this contact (Glaser, 1969; Melo, 1988; Grahn et al., 2010a). The Unit 3 presents the Neoemsian-Eifelian age (Grahn et al., 2010a). On the other hand the São Domingos Formation (Apucarana Sub-basin) has predominantly clay shales of Neoemsian-Eofrasnian age (Melo and Loboziak, 2003; Grahn et al., 2013).

Chapada Group Unit 4 usually consists of dark gray shales with intercalations of clay sandstones and siltstones. The upper contact of this unit is erosive and occurs with the diamictites of the Itararé Group (Carboniferous) (Andrade and Camarço, 1980; Grahn et al., 2010a) (Fig. 2). This unit is correlated to the upper portion of the São Domingos Formation (Apucarana Sub-basin; Grahn et al., 2013), and the beginning of its deposition is related to the maximum transgression, which occurred in the early Givetian (Assine, 2001; Grahn et al., 2010a). According to Grahn et al. (2010a, 2013), the rocks of this unit have Givetian-Eofrasnian age.

\section{MATERIAL AND METHODS}

The new specimens of Metacryphaeus tuberculatus from the Paraná Basin were collected in the Ponta Grossa Formation (Apucarana Sub-basin), and Chapada Group Unit 4 (Alto Garças Subbasin). Samples from Unit 4 were collected in the surroundings of Doverlândia city (16²'43.080'S, 52 ${ }^{\circ} 15^{\prime} 46.897^{\prime}$ 'W, Goiás State, Brazil) in association with tentaculitids, brachiopods, ichnofossils, and plant remains (Figs. 1 and 3). This material was deposited in the scientific collection of Laboratório de Paleontologia de Macroinvertebrados (LAPALMA) located at Universidade Estadual Paulista (UNESP), Bauru Campus, under the numbers CCLP 874a and CCLP 874b.

The specimen of $M$. tuberculatus from the upper part of the Ponta Grossa Formation was previously classified in the unpublished thesis of Popp (1985) as M. granulata and it is deposited in the Universidade Federal do Paraná (UFPR) collection under the number NR3130. 


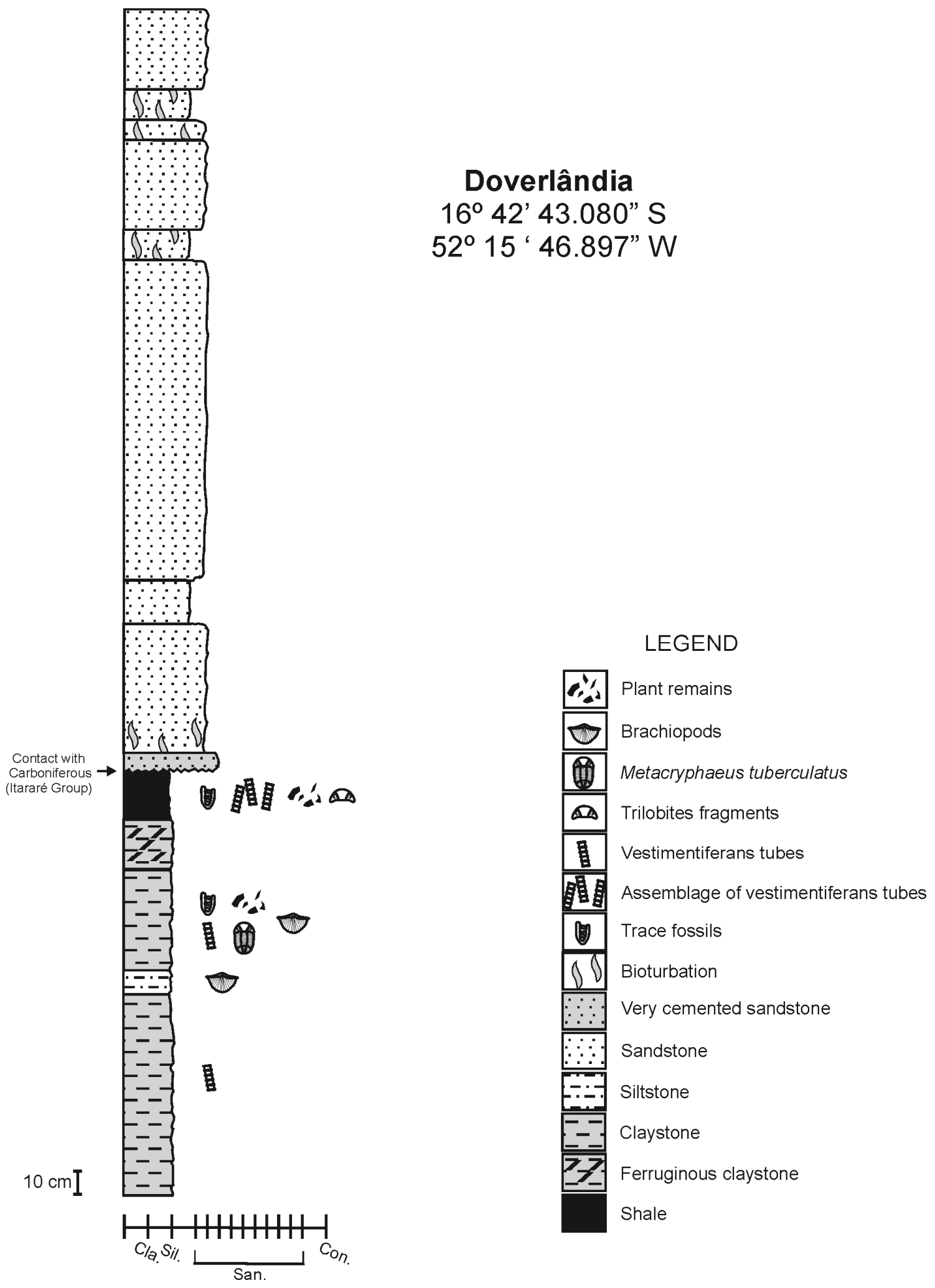

Figure 3. Columnar profile of the Doverlândia outcrop (Chapada Group Unit 4).

The rocks of São Domingos Formation where $M$. australis was collected present the Givetian age (Melo and Loboziak, 2003; Grahn et al., 2013) and are from two outcrops located near 
Tibagi city (Paraná State, Brazil). The Xaxim outcrop is located on the BR 153 highway (km 237) and the Amparo outcrop is found at the Alto do Amparo entrance (Fig. 2). This material is deposited in the collection of Laboratório de Estratigrafia e Paleontologia of Departamento de Geociências of Universidade Estadual de Ponta Grossa under the numbers DEGEO/MPI 1535, DEGEO/MPI 1536, DEGEO/MPI 1539 A, DEGEO/MPI 1539 B, DEGEO/MPI 1543, DEGEO/MPI 1555, DEGEO/MPI 1556, DEGEO/MPI 1557, and DEGEO/MPI 8476 B.

\section{SYSTEMATIC PALEONTOLOGY}

Order PHACOPIDA Salter, 1864

Family CALMONIIDAE Delo, 1935

Subfamily CALMONIINAE Delo, 1935

Genus Metacryphaeus Reed, 1907

Type species. Metacryphaeus caffer (Salter, 1856)

Generic diagnosis. Rounded anterior margin, may present a small frontal process. Cephalic region slightly arched in most species. Axial furrows narrow and moderately divergent. Inflated frontal lobe. Glabellar furrows S3 well defined, reduced in S2, and deepest in S1. S1 may reach the axial furrows. Glabellar lobes L2 and L3 connected sporadically. Thoracic axial portion wide. Thoracic pleurae with sharp edges. Pygidium with 8 to 11 rings, 5 pairs of pleural ribs, deep pleural furrows, narrow inter-pleural furrows, and 5 pairs of pleural lapels.

Metacryphaeus tuberculatus (Kozlowski, 1923)

Figure 4. 1-5

1923. Cryphaeus australis var. tuberculatus Kozlowski, p. 43, pl. 3, figs. 20-21.

1965. Metacryphaeus caffer var. Braniša, p. 106, pl. 21, figs. 14, 16.

1968. Metacryphaeus tuberculatus Wolfart, p. 102, pl. 17, fig. 17, pl. 18, fig. 1.

1991. Metacryphaeus tuberculatus Lieberman et al., p. 825-827, fig. 1.1.

Lectotype. MNHN.F.R50817 (Kozlowski, 1923, p. 43-44, pl. 3, fig. 20). 
Examined material. CCLP 874a, CCLP 874b, and NR3130.

Geographic and stratigraphic provenance. Upper Belén and Sicasica formations of the La Paz Department (Bolivia), Icla Formation of the Chuquisaca Department (Bolivia), Limoncito Formation of the Santa Cruz Department (Bolivia), Pimenteira Formation of the Parnaíba Basin (Piauí State, Brazil). In the present work, CCLP 874a and CCLP 874b correspond to Chapada Group Unit 4 (Givetian-Frasnian, Alto Garças Sub-basin, Paraná Basin). NR3130 is from the upper part of the Ponta Grossa Formation (Early Emsian, Apucarana Sub-basin, Paraná Basin).

Description. Concave and convex external molds of cephalon (CCLP 874a, CCLP 874b, and NR3130). Cephalic dimensions $1.7 \mathrm{~cm}$ in length and $3.4 \mathrm{~cm}$ in width in CCLP 874a and CCLP 874b. NB3130 is $2.2 \mathrm{~cm}$ in length and $4.5 \mathrm{~cm}$ in width. In other words, the cephalic length is equal to $50 \%$ of its width. Cephalic length is equal to $90 \%$ of frontal glabellar lobe width. The values of LEI were $0,29-0,31$ in the analyzed specimens. The cephalon presents a sub-triangular shape with tubercles throughout the glabella, glabellar lobe, palpebral portion, and extra-ocular area. These tubercles also occur on the occipital lobe (L0) and the proximal half of the posterior border (Fig. 4). The frontal glabellar lobe is inflated and laterally expanded exhibiting a posterior median impression. The glabellar area is sub-trapezoidal and relatively convex. The frontal glabellar lobe length is $60 \%$ of the glabellar length. Axial furrows start on the top of S3, are well defined, slightly curved antero-distally, deep, and oblique, and they diverge toward the anterodistal region. The most well-defined lateral glabellar furrow is S3, which is slightly curved and markedly delimits the frontal lobe. The S3's depth decreases as it meets the axial furrow. S2 is well marked, deep (although less than S3 and S1), wider, and curved where it contacts the axial furrow, and narrower and straight toward the glabellar center. S1 is also deep, well-marked, and in contact with the axial furrow (more defined than the connection with S2). S1 is curved anteriorly in its proximal margins. The proximal portion of $\mathrm{S} 1$ is very close to the proximal portion of S2 and almost contacts it. S0 (occipital furrow) is deep, well-marked, sometimes curved anteriorly, and connects with the axial furrows and posterior border furrows. S0 becomes deeper and narrower distally. Glabellar furrows are inflated and have different shapes. L3 is subtriangular to sub-trapezoidal. L2 is oblong. L1 is concave anteriorly. L0 (occipital lobe) is prominent, and its distal portion is slightly arched forward. L0 corresponds to $70 \%$ of the width of the frontal glabellar lobe. The eyes are relatively small, reniform, and separated from the axial furrows. Some lenses are preserved on the anterior margin of the eyes, indicating schizochroal eyes. The anterior margin of the eyes is at the same level as the contact between the axial furrow and S3. The posterior margin is at half the height of L2. Facial suture proparian. Lateral cephalic 
border furrow shallow. Posterior cephalic border furrow well-marked and laterally follows the posterior border of the cephalon, curving anteriorly and becoming shallower distally. There is a connection between the posterior cephalic border furrow and the lateral cephalic border furrow. Lateral cephalic border narrow, convex, and in contact with the posterior cephalic border. Genal margins curved anteriorly. Genal spines absent. Posterior cephalic border oblique (in relation to axial furrows) and curved forward distally. Posterior cephalic border wider distally and narrower at the point of contact with the axial furrow.

Discussion. The taxonomic classification of some species of Metacryphaeus is difficult because the species differences are subtle, as can be seen in M. tuberculatus and M. caffer (Lieberman et al., 1991). However, the main features used to identify Metacryphaeus tuberculatus in the Paraná Basin are cephalic features such as furrows and glabellar lobes, LEI, and tuberculation patterns.

The schizochroal eyes identified on CCLP 874a and CCLP 874b are exclusive to the suborder Phacopina and are related to the predatory habit of these organisms (Fig. 4.4) (Clarkson et al., 2006; Fortey, 2014).

NR3130 corresponds to the sample classified by Popp (1985, unpublished thesis) as Metacryphaeus granulata (nom. nud.). This sample was collected in the upper part of the Ponta Grossa Formation (Early Emsian, Apucarana Sub-basin, Paraná Basin, Paraná State, Brazil). Nevertheless, we consider that NR3130 belongs to $M$. tuberculatus on the basis of its glabellar furrows, glabellar lobes, and tuberculation pattern (Fig. 4.5).

M. sedori (nom. nud.) as described by Popp (1985) resembles M. tuberculatus, but M. sedori is clearly worn due to taphonomic processes. In addition, M. sedori is more similar to M. australis in its glabellar lobes and glabellar furrows.

According to Lieberman et al. (1991), one of the main differences between M. australis, M. caffer, and M. tuberculatus is the LEI which consists in the ratio between the eye length and the length of the glabella without consider the occipital furrow (Wolfart, 1968). However, the values of this index presents subtle differences between these species and, in some cases, can be overlaid in part as in case of $M$. caffer and M. australis (0,29-0,39 and 0,38-0,41, respectively). Although, M. tuberculatus shows lower values of LEI $(0,26-0,30)$ than the other species, just with a subtle overlay in relation to the LEI of $M$. caffer. Due to this, LEI can be used to differentiate M. tuberculatus from M. caffer and M. australis, but it does not clearly distinguish 
the latter two species. The values of LEI of the individuals analyzed are 0,29-0,31 which correspond to the range of $M$. tuberculatus.

Besides the LEI, Metacryphaeus tuberculatus differs from M. australis (Clarke, 1913), from Ponta Grossa and São Domingos formations (Apucarana Su-basin, Paraná Basin, Brazil) and M. caffer (Salter, 1856), from Bokkeveld Group (South Africa), with respect to S2, which in $M$. australis and $M$. caffer does not connect or subtly connect with the axial furrow. It also differs with respect to $\mathrm{S} 1$, which in $M$. australis and $M$. caffer makes tenuosus contact with the axial furrow (but more detached than the contact between the axial furrow and S2). The glabellar furrows are less marked in M. australis and M. caffer. L3 may weakly connect with L2 distally in M. australis and M. caffer. L2 and L1 shapes are sub-rectangular in M. australis and M. caffer. Glabellar lobes in M. australis and M. caffer are not inflated as in M. tuberculatus. The anterior margin of $M$. tuberculatus eyes does not reach the axial furrow as it does in M. australis and $M$. caffer. The tuberculation of M. tuberculatus is salient, while in M. australis it is finer and denser. According to Lieberman et al. (1991), the tuberculation difference between these species is not related to taphonomic processes because there are $M$. australis samples in excellent preservational conditions showing fine and dense tuberculation.

The main differences between M. tuberculatus and M. rotundatus (Kozlowski, 1923), described from Emsian rocks of the Icla Formation (Bolivia) and the Ponta Grossa Formation (Apucarana Sub-basin, Paraná Basin, Brazil), are in the genal spine, which is long and thin in M. rotundatus; in the absence of tubercles in M. rotundatus; in the relation between glabellar length and frontal lobe width, which is smaller in M. rotundatus (85\%); in the length/width ratio of the frontal lobe, which is bigger in M. rotundatus (55\%); in S2 shape, which is narrower in proximal and distal portions in $M$. rotundatus; and in the position of the eyes on the cephalon, which have their posterior and anterior margins closer to the axial furrows in M. rotundatus.

Comparing to calmoniids from Paraníba Basin (Brazil), M. tuberculatus differs from M. kegeli Carvalho et al., 1997 from the Eifelian of the Pimenteira Formation in the absence of shallow depressions on the librigenal field and extra-ocular fixigena. Axial and glabellar furrows are more marked in M. kegeli. The distance between the eyes is wider in M. kegeli and the shape of these structures is also distinct.

M. tuberculatus and M. meloi Carvalho et al., 1997 from the Passagem Member (Givetian, Pimenteira Formation, Parnaíba Basin, Brazil) are very similar, except with respect to their eye size. According to Carvalho et al. (1997), the characters that distinguish M. meloi from M. 
tuberculatus are posterior medium impression less defined in $M$. meloi, axial furrows and S3 narrower in $M$. meloi, tuberculation less evident in M. meloi, and lateral glabellar lobes less conspicuous in M. meloi.

M. tuberculatus and M. giganteus (Ulrich, 1892) from the Devonian of the Icla and Belén formations (Bolivia) and the Cabanillas Formation (Peru) differ in the format and position of the eyes (longer and with anterior and posterior margins closer to the axial furrows in M. giganteus); in the axial furrow, glabellar furrow, and posterior border furrow (more prominent in $M$. giganteus); in L2 shape (proximal portion is more curved anteriorly in M. giganteus); in L1 shape (narrower in M. giganteus); in the frontal glabellar lobe (more expanded anteriorly in $M$. giganteus); and in the genal spines (long and narrow in M. giganteus).

M. tuberculatus differs from M. convexus (Ulrich, 1892), from the Icla Formation (Bolivian Devonian), because in $M$. convexus, the anterior margin of the eyes is connected to the axial furrow; the glabella is more inflated; L2 and L3 are more inflated and lager; the axial furrows, glabellar furrows, occipital furrow, and posterior border furrows are more marked; the proximal portion of $\mathrm{S} 1$ is more curved anteriorly; L1 is narrower and smaller; the frontal lobe more inflated; and the tuberculation pattern distinct.

The main differences between M. tuberculatus and M. curvigena Lieberman, 1993, from the Icla Formation (Bolivian Devonian), are the axial furrow and posterior border furrows, which are more marked in $M$. curvigena; L1 is narrower in $M$. curvigena; $\mathrm{S} 1$ is more marked in $M$. curvigena; the posterior median impression is less prominent in M. curvigena; L2 and L3 are more inflated and seem to be a single structure in M. curvigena; the genal spine of $M$. curvigena is thin and curved inwards on the terminal portion; the anterior margin of the eyes is connected to the axial furrow in $M$. curvigena; and the posterior margin is nearer to the axial furrow in $M$. curvigena.

Compared to M. branisai Lieberman, 1993, from the Icla Formation (Devonian, Bolivia), M. tuberculatus has a more prominent posterior median impression, L1 is wider, S3 is more marked, L0 is less inflated, and the antero-median portion of the lateral cephalic border of M. branisai is flexed antero-distally.

M. tuberculatus and M. allardyceae (Clarke, 1913), from the Fox Bay Formation (Falkland Islands), differ in that $M$. allardyceae presents a very long frontal spine and eyes nearer to the lateral cephalic border. 


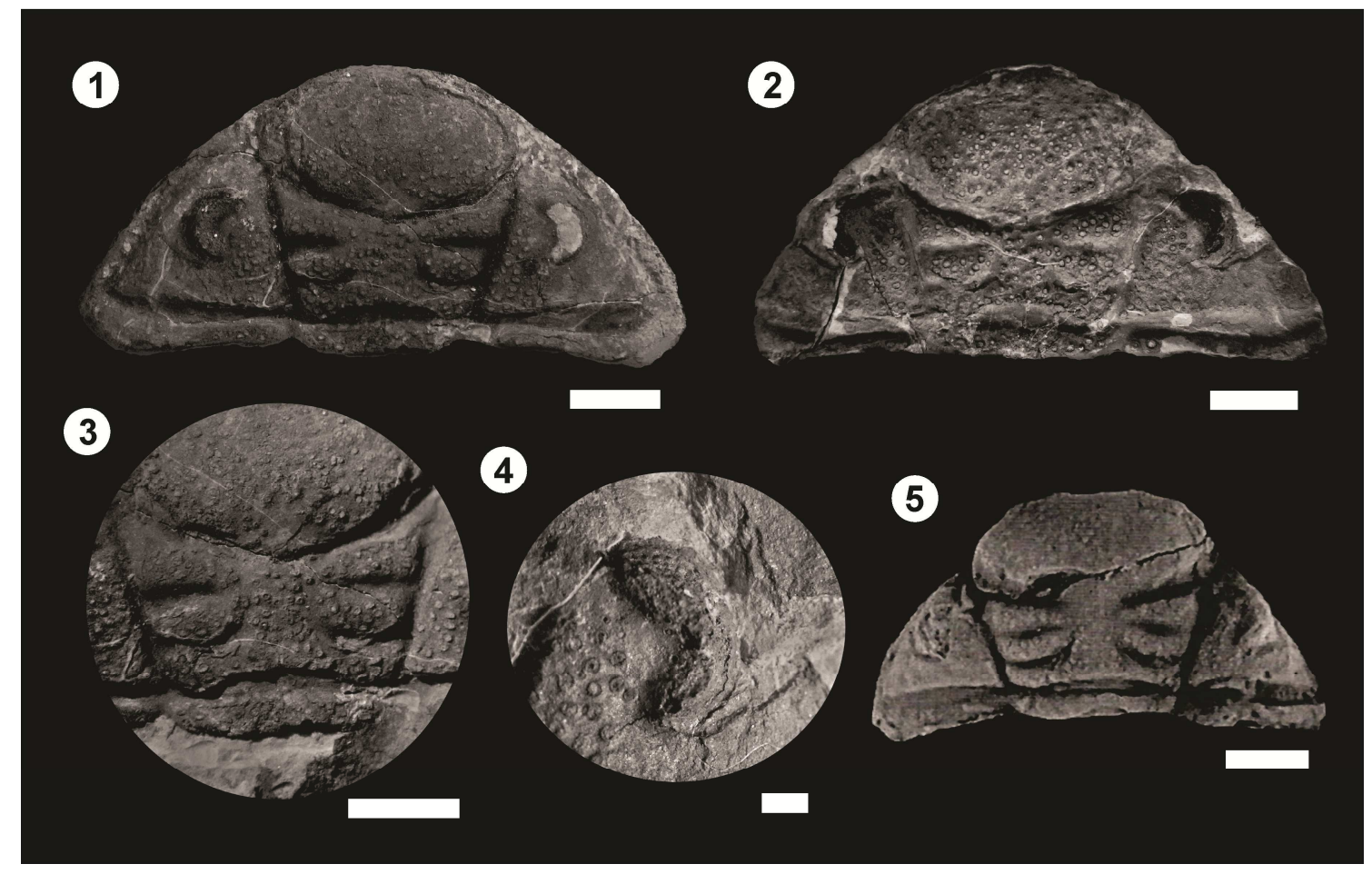

Figure 4. Metacryphaeus tuberculatus. 1, external convex mold of cephalon in dorsal view (CCLP 874a); 2, external concave mold in dorsal view (CCLP 874b); 3, details of the glabellar lobes and furrows (CCLP 874a); 4, eyes with preserved lenses (CCLP 874b); 5, cephalon in dorsal view (NR3130; image taken from Stasi, 2013). Scale bar: 1, 2, 3, 5=5 mm; $4=1 \mathrm{~mm}$.

Metacryphaeus australis (Clarke, 1913)

Figure 5. 1-4

1913. Cryphaeus australis Clarke, p. 108-114, pl. 3, figs. 7-14; pl. 4, figs. 1-5.

1925. Dalmanites australis Reed, p.145, pl. 11, fig. 9.

1935. Hadrorachus australis (Clarke) Delo, p. 415, figs. 34, 35.

1942. Asteropyge australis Richter and Richter, p. 134.

Lectotype. DGM 35-5 (Clarke 1913, pl. 4, fig. 5) 
Examined material. DEGEO/MPI 1535, DEGEO/MPI 1536, DEGEO/MPI 1539 A, DEGEO/MPI 1539 B, DEGEO/MPI 1543, DEGEO/MPI 1555, DEGEO/MPI 1556, DEGEO/MPI 1557, and DEGEO/MPI 8476 B.

Geographic and stratigraphic provenance. Ponta Grossa and São Domingos formations (Apucarana Sub-basin, Paraná Basin, Paraná State, Brazil). The material presented here was collected in the rocks of the São Domingos Formation (Givetian age, Apucarana Sub-basin, Paraná Basin).

Description. Cephalic portion incomplete (DEGEO/MPI 1556; Fig. 5.1). Cephalic dimensions $2.25 \mathrm{~cm}$ in length and $4.5 \mathrm{~cm}$ in width. Cephalic length is equal to $90 \%$ of frontal glabellar lobe width. The estimated value of LEI is 0.40 . Small tubercles are present in small amounts. Posterior median impression present. The frontal glabellar lobe length is $55 \%$ of the glabellar length. Axial furrow well marked. Lateral glabellar furrow less marked than axial furrow. S3 is more marked than the others lateral glabellar furrows. S2 does not connect with the occipital furrow. S1 slightly curved in its proximal portion. S1 connects the axial furrow distally. S0 is well marked and deep. The glabellar lobes presents different shapes. L3 is sub-trapezoidal. L2 is sub-rectangular. L1 is sub-rectangular with slight concavity in its anterior portion. The glabellar lobe (L0) is well marked. Only an eye was preserved with relatively big size, reniform shape, and with a connection between the anterior margin of the eye and the axial furrow. Thoracic portion incomplete (DEGEO/MPI 1535, DEGEO/MPI 1536, DEGEO/MPI 1555, and DEGEO/MPI 8476 B; Fig. 5.2). Pygidium (DEGEO/MPI 1539 A, DEGEO/MPI 1539 B, DEGEO/MPI 1543 and DEGEO/MPI 1557; Figs. 5.3 and 5.4) sub-triangular in shape, moderately convex with evident tuberculation. Anterior axial portion wider than posterior portion and tapering towards posterior portion. After the five anterior axial rings of the pygidium, axial furrows become sub-parallel. Approximately 11 axial rings. Pleural spines (marginal) of pygidium blade-shaped.

Discussion. The diagnostic characters used to identify this species were the LEI, S1 (without connection to the axial furrow), S2 (little sinuous), tuberculation (less dense), the connection of the anterior margin of the eyes with the axial furrow, and the pygidium (with 5 pairs of pleural lappets). The features of the axial furrows could be interpreted as alterations caused by taphonomic processes, but the cephalon here presented is relatively well preserved which could be observed by the presence of tuberculations in this structure and some lens in the eyes. The presence of these lens indicates schizochroal eyes. 
Between the species of Metacryphaeus genus, the most similar to M. australis are $M$. tuberculatus e M. caffer. However, M. australis differs from M. tuberculatus by the LEI, the tuberculation pattern, and the glabellar furrows. On the other hand, the diferences between $M$. australis and M. caffer are subtle. M. australis presents slightly higher values of LEI (0,29-0,39 in M. caffer and 0,38-0,41 in M. australis). However, M. caffer has a bigger range of LEI.

It is notable the similarities between $M$. australis and $M$. caffer which makes important the realization of a more accurate systematic review of these species.

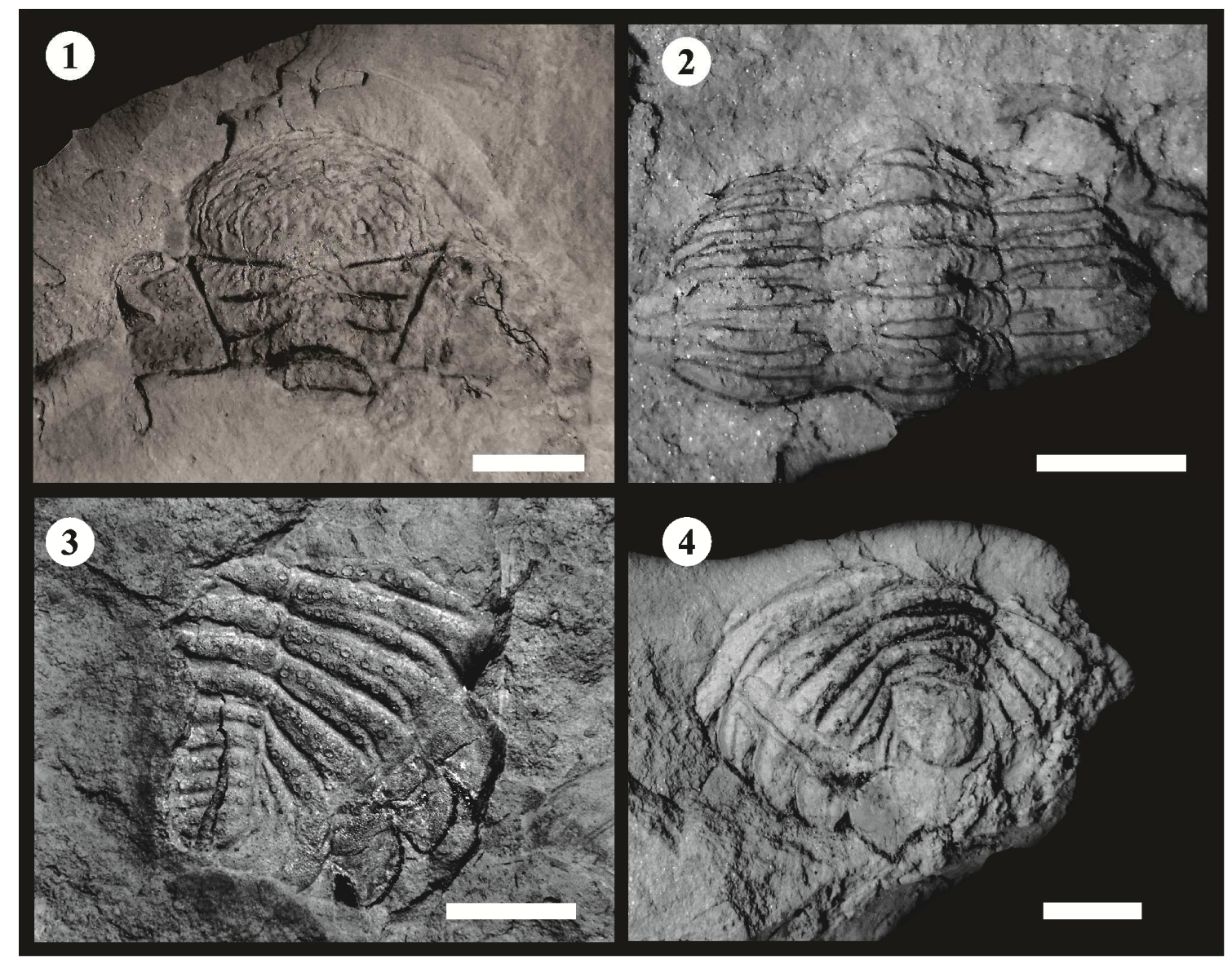

Figure 5. Metacryphaeus australis. 1, external convex mold in dorsal view (DEGEO/MPI 1556).

2, thoracic portion in dorsal view (DEGEO/MPI 1536). 3-4, pygidium in dorsal view (DEGEO/MPI 1557, DEGEO/MPI 1539 B). Scale bar= 5 mm.

\section{FOSSILS ASSOCIATED WITH M. TUBERCULATUS AT DOVERLÂNDIA OUTCROP (GOIÁS STATE)}


The record of $M$. tuberculatus in the Chapada Group Unit 4 is associated with a claystone bed which presents tiny brachiopods, vestimentiferans tubes (quite commons in the end of the Middle Devonian) (Figs. 3, 6.7, and 6.8) (Bosetti et al., 2011, 2014), ichnofossils, and plant remains. Among the fossils mentioned above, the vestimentiferans tubes are the unique present in large amounts.

The brachiopods found were identified as Derbyina, indeterminate Chonetidina, and probable indeterminate Obolidae of small dimensions that can vary between 1-7 mm of length (Fig. 6.1$5)$.

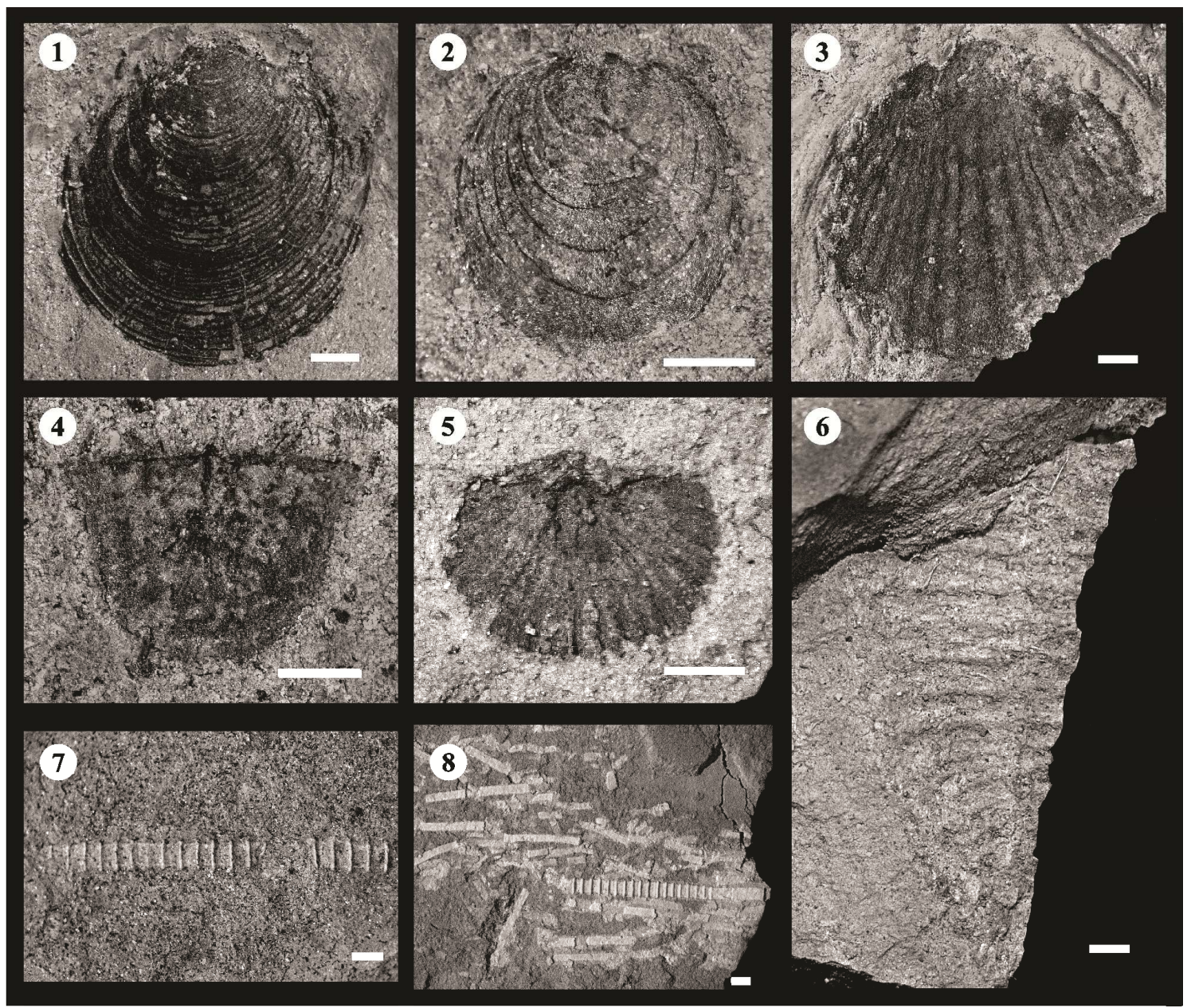

Figure 6. Some fossils found in association with M. tuberculatus at Doverlândia (GO) outcrop. 1-2, indeterminate Obolidae. 3, Derbyina sp. 4, indeterminate Chonetidina. 5, indeterminate brachiopod. 6, trilobite remains. 7, vestimentiferan tube. 8, assemblage of vestimentiferans tubes. Scale bar: $\mathbf{1 , 2} \mathbf{2}, \mathbf{7}, \mathbf{8}=1 \mathrm{~mm} ; \mathbf{2}, \mathbf{4}, \mathbf{5}=0,5 \mathrm{~mm}$. 
Although the $M$. tuberculatus cephalon does not show small dimensions, another fragmented trilobite was found with small size (about $10 \mathrm{~mm}$ in length without cephalic portion) (Fig. 6.6).

The vestimentiferans tubes are preserved in assemblage at the top of the columnar profile (near to the contact with the Carboniferous of Itarare Group; Figs. 3 and 6.8). However, these fossils also occur in more basal beds of the outcrop but not in assemblage.

\section{PALAEOBIOGEOGRAPHY}

Metacryphaeus presents occurrences in many areas of Gondwana and in different Devonian geological units. M. australis is endemic of Paraná Basin and occurs in Ponta Grossa and São Domingos formations in Apucarana Sub-basin (Clarke, 1913; Popp, 1987) and in units 2 or 3 (Emsian-Eifelian) and 4 (Givetian) of Chapada Group in Alto Garças Sub-basin (Carvalho et al., 1987; Carvalho and Edgecombe, 1991). On the other hand, comparing to the other Metacryphaeus sepecies, M. tuberculatus presents more records which are distributed in geological units localized in Bolivia, Peru, and Brazil, showing a cosmopolite tendency.

The bolivian occurrences of $M$. tuberculatus includes Icla Formation (Praguian-Emsian), the upper part of Belén Formation (Emsian-Eifelian), Sicasica Formation (Eifelian), and Limoncito Formation (?Neopraguian-?Givetian) (Lieberman et al., 1991; Lieberman, 1993). The peruvian records just involves Cabanillas Formation (Early Devonian) (Velazco, 2012). The brazilian records include Paraná Basin (Eoemsian of Ponta Grossa Formation, Apucarana Sub-basin; Givetian-Eofrasnian of Chapada Group Unit 4, Alto Garças Sub-basin) (in the present work) and Parnaíba Basin (Neoeifelian-Eogivetian of Pimenteira Formation) (Lieberman et al., 1991; Lieberman, 1993).

The new records of $M$. tuberculatus in the Emsian of Paraná Basin instigate a different palaeobiogeographic interpretation of that known for the brachiopods of the genus Tropidoleptus and the crinoid Exaesiodiscus (Melo, 1985; Fonseca and Melo, 1987; Melo, 1988; Petri and Fulfaro, 1988; Scheffler, 2010; Scheffler et al., 2011).

M. tuberculatus was previously recorded in Brazil in the Neoeifelian-Eogivetian of Pimenteira formation (Parnaíba Basin) and in some geological units of Peru and Bolivia. However, this new occurrence in the Eoemsian of Apucarana Sub-basin (Paraná Basin) implies that M. tuberculatus would have dispersed between Bolivia, Peru, and Brazil during the Emsian transgression. Later, 
this species probably migrates to Alto Garças Sub-basin (Paraná Basin) and Parnaíba Basin during the Givetian transgression.

On the other hand, it is also possible that the arrival of M. tuberculatus in the Parnaíba Basin would have occured through a dispersive event that occurrs between Bolivia, Amazon Basin (Brazil), and Parnaíba Basin (Brazil). The connection between these localities is already known in the literature by the fossils of Tropidoleptus, Exaesiodiscus, Laudonomphalus, Monstrocrinus, and Marettocrinus (Fonseca and Melo, 1987; Melo, 1988; Petri and Fulfaro, 1988; Assine, 2001; Scheffler, 2010; Scheffler et al., 2011). However, there is no records of the presence of $M$. tuberculatus and the genus Metacryphaeus in the Amazon Basin.

\section{CONCLUSIONS}

A new occurrence of M. tuberculatus is recorded in the Paraná Basin (Brazil), both in Alto Garças and Apucarana sub-basins. This record is important for future palaeogeographic contributions. This new record is presented jointly with new findings of M. australis in the Apucarana Sub-basin, which were used to show the differences between M. australis and M. tuberculatus.

M. tuberculatus, M. australis, and $M$. caffer have some morphological similarities but the main differences are observed in the glabellar furrows S1 and S2, glabellar lobes L1, L2, and L3, tuberculation pattern, the position of the eyes in relation to the axial furrow, and in the LEI.

The presence of M. tuberculatus in the Paraná Basin instigates a different palaeogeographical interpretation of that presented by Tropidoleptus and Exaesiodiscus (Fonseca and Melo, 1987; Melo, 1988; Petri and Fulfaro, 1988; Scheffler, 2010; Scheffler et al., 2011). M. tuberculatus would has arisen in the Lower Devonian and habited areas as Bolivia, Peru, and Brazil (Subbacia Apucarana, Paraná Basin). It is probable the migration of M. tuberculatus between these areas during the Emsian transgression. In the Givetian, M. tuberculatus would has migrated to Alto Garças Sub-basin (Paraná Basin) and Parnaíba Basin where they have registrations.

Differently of the palaeobiogeographical pattern presented by M. tuberculatus, M. australis is endemic of Paraná Basin and presents occurrences both in Apucarana and Alto Garças subbasins. 
Metacryphaeus has representatives in all Devonian stages and includes a large number of species distributed in various regions of Gondwana as Bolivia, Peru, Brazil, the Falkland Islands, and South Africa. However, the genus still needs to be taxonomically revised in order for these contributions to be used in future palaeobiogeographical and palaeoecological analyses.

\section{ACKNOWLEDGEMENTS}

F. A. Carbonaro would like to thank Fundação de Amparo à Pesquisa do Estado de São Paulo (FAPESP 2013/09683-3) for financial support, the biologist Bruno Francisco dos Santos, and all LAPALMA team because of the collaboration in field works at Goiás State (Brazil). F. van E. Meira wishes to thank Fundação de Amparo à Pesquisa do Estado de São Paulo (FAPESP 2012/07075-3) for financial support. E. P. Bosetti would like to thank Conselho Nacional de Desenvolvimento Científico e Tecnológico (CNPq 311483/2014-3) for financial support.

\section{REFERENCES}

Andrade, S.M. and Camarço, P.E.N. 1980. Estratigrafia dos sedimentos devonianos do flanco nordeste da Bacia do Paraná. XXXI Congresso Brasileiro de Geologia (Balneário de Camboriú), Anais 5: 2828-2834.

Assine, M.L. 2001. O Ciclo Devoniano na Bacia do Paraná e correlação com outras bacias Gondwânicas. Ciência, Técnica, Petróleo, Seção Exploração de Petróleo 20: 55-62.

Assine, M.L.; Perinotto, J.A.J.; Fulfaro, V.J. and Petri, S. 1998. Progradação deltáica Tibagi no Devoniano Médio da Bacia do Paraná. Revista Brasileira de Geociências 28 (2): 125-134.

Baldis, B. and Longobucco, M. 1977. Trilobites devonicos de la Precordillera Noroccidental (Argentina). Ameghiniana 14: 145-161.

Bosetti, E.P.; Grahn, Y.; Horodyski, R.; Mendlowicz Mauller, P. 2012. The first recorded decline of the Malvinokaffric Devonian fauna in the Paraná Basin (southern Brazil) and its cause; taphonomic and fossil evidences. Journal of South American Earth Sciences 37: 228-241. 
Bosetti, E. P.; Grahn, Y.; Horodyski, R.S.; Mendlowics Mauller, P.; Breuer, P. and Zabini, C. 2011. An earliest Givetian "Lilliput Effect" in the Paraná Basin, and the collapse of the Malvinokaffric shelly fauna. Paläontologische Zeitschrift 85: 49-65.

Bosetti, E.P.; Horodyski, R.S.; Comniskey, J.C.; Ghilardi, R.P. and Sedorko, D. 2014. Tubos vestimentíferos no Eogivetiano da Formação São Domingos, Bacia do Paraná?. II Simpósio Brasileiro de Paleoinvertebrados (Ponta Grossa), Paleontologia em Destaque - Boletim de Resumos, Edição Especial: 107.

Boucot, A.J. 1971. Malvinokaffric Devonian marine community distribution and implications for Gondwana. Anais da Academia Brasileira de Ciências, Suplemento 43: 23-49.

Boucot, A.J. 1974. Silurian and Devonian biogeography. In: C.A. Ross (Ed.), Paleogeography provinces and provinciality, Society of Economic Mineralogists and Paleontologists, Special Publication, 21: 165-176.

Boucot, A.J. 1985. Late Silurian-early Devonian biogeography, provincialism, evolution and extinction. Philosophical Transaction of the Royal Society of London, B, 309 (1138): 323339.

Braniša, L. 1965. Los fossiles guias de Bolivia. I. Paleozoico. Servicio Geologico de Bolivia, Boletin 6: 1-282.

Carvalho, M.G.P. and Edgecombe, G.D. 1991. Lower-Early Middle Devonian Calmoniid Trilobites from Mato Grosso, Brazil, and Related Species from Parana. American Museum Novitates 3022: 1-13.

Carvalho, M.G.P., Edgecombe, G.D. and Lieberman, B.S. 1997. Devonian Calmoniid trilobites from the Parnaíba Basin, Piauí State, Brazil. American Museum Novitates 3192: 1-11.

Carvalho, M.G.P., Melo, J.H.G. and Quadros, L.P. 1987. Trilobitas devonianos do flanco noroeste da bacia do Parana. X Congresso Brasileiro de Paleontologia (Rio de Janeiro), Anais 2: 545-565.

Clarke, J.M. 1913. Fósseis devonianos do Paraná. Monographias do Serviço Geológico e Mineralógico do Brasil 1: 1-353.

Clarkson, E., Levi-Setti, R. and Horváth, G. 2006. The eyes of trilobites: the oldest preserved visual system. Arthropod Structure \& Development 35: 247-259. http://dx.doi.org/10.1016/j.asd.2006.08.002 
Cooper, M.R. 1982. A revision of the Devonian (Emsian-Eifelian) Trilobita from the Bokkeveld Group of South Africa. Annals of the South African Museum 89: 1-174.

Delo, D.M. 1935. A revision of the phacopid trilobites. Journal of Paleontology 9: 402-420.

Eldredge, N. and Ormiston, L. 1979. Biogeography of Silurian and Devonian trilobites of the Malvinokaffric Realm. In: A.J Boucot and J. Gray (Eds.), Historical Biogeography, plate tectonics and the changing environment, Oregon State University Press, Corvalis, p. 147167.

Fonseca, V.M.M. and Melo, J. H. G. 1987. Ocorrência de Tropidoleptus carinatus (Conrad) (Brachiopoda, Orthida) na Formacão Pimenteira, e sua importância paleobiogeográfica. X Congresso Brasileiro de Paleontologia (Rio de Janeiro), Anais 2: 505-537.

Fortey, R.A. 2014. The palaeoecology of trilobites. Journal of Zoology 292: 250-259. http://dx.doi.org/10.1111/jzo.12108

Ghilardi, R.P. and Simões, M.G. 2007. History and development of trilobite research in Brazil. In: D.G. Mikulic, E. Landing, and J. Kluessendorf (Eds.), Fabulous Fossils - 300 years of worldwide research on trilobites, New York State Museum Bulletin, 507, p. 97-104.

Glaser, I. 1969. A formação Furnas no SW de Goiás. XXIII Congresso Brasileiro de Geologia, Anais, p. 135-144.

Grahn, Y., Mendlowicz Mauller, P.; Bergamaschi, S. and Bosetti, E.P. 2013. Palynology and sequence stratigraphy of three Devonian rock units in the Apucarana Sub-basin (Paraná Basin, south Brazil): Additional data and correlation. Review of Palaeobotany and Palynology 198: 27-44. http://dx.doi.org/10.1016/j.revpalbo.2011.10.006

Grahn, Y., Mendlowicz Mauller, P., Pereira, E. and Loboziak, S. 2010a. Palynostratigraphy of the Chapada Group and its significance in the Devonian stratigraphy of the Paraná Basin, south Brazil. Journal of South American Earth Sciences 29: 354-370. http://dx.doi.org/10.1016/j.jsames.2009.09.001

Grahn, Y., Mendlowicz Mauller, P., Breuer, P., Bosetti, E.P., Bergamaschi, S., Pereira, E. 2010 b. The Furnas/Ponta Grossa contact and the age of the lowermost Ponta Grossa Formation in the Apucarana Sub-basin (Paraná Basin, Brazil): integrated palynological age determination. Revista Brasileira de Paleontologia 13: 89-102. http://dx.doi.org/10.4072/rbp.2010.2.02

Kozlowski, R. 1923. Faune devonienne de Bolivie. Annales de Paléontologie 12: 1-112. 
Lieberman, B.S. 1993. Systematics and biogeography of the "Metacryphaeus Group", Calmoniidae (Trilobita, Devonian), with comments on adaptive radiations and the geological history of the Malvinokaffric Realm. Journal of Paleontology 67 (4): 549-570.

Lieberman, B.S., Edgecombe, G. D. and Eldredge, N. 1991. Systematics and biogeography of the "Malvinella group", Calmoniidae (Trilobita, Devonian). Journal of Paleontology 65: $824-843$.

Melo, J.H.G. 1985. [A Província Malvinocáfrica no Devoniano do Brasil: estado atual dos conhecimentos. Tese de doutorado, Universidade Federal do Rio de Janeiro, Instituto de Geociências, 3 vol., 890 p. Unpublished.]

Melo, J.H.G. 1988. The Malvinokaffric Realm in the Devonian of Brazil. In: N.J. McMillan, A.F. Embry, and D.J. Glass (Eds.), Devonian of the World, Canadian Society of Petroleum Geologists (Memoir 14), p. 669-703.

Melo, J.H.G. and Loboziak, S. 2003. Devonian-Early Carboniferous miospore biostratigraphy of the Amazon Basin, Northern Brazil. Review of Palaeobotany and Palynology 124: 131202. http://dx.doi.org/10.1016/S0034-6667(02)00184-7

Milani, E.J.; Melo, J.H.G.; Souza, P.A.; Fernandes, L.A. and França, A.B. 2007. Bacia do Paraná. Boletim de Geociências da Petrobrás 15 (2): 265-287.

Petri, S. and Fulfaro, V.J. 1988. Geologia do Brasil. 2a ed. São Paulo, T.A. Queiroz/Ed. da Universidade de São Paulo, v. 9, 631 p.

Popp, M.T.B. 1985. [Revisão dos trilobitas calmoniideos e comunidades faunísticas da Formação Ponta Grossa, Devoniano do Estado do Paraná Brasil. Tese de doutorado, Universidade Federal do Rio Grande do Sul, Porto Alegre, 112 p. Unpublished.]

Ramos, A.N. 1970. Aspectos paleo-estruturais da Bacia do Paraná e sua influência na sedimentação. Boletim Técnico da Petrobrás 13 (3-4): 85-93.

Reed, F.R.C. 1907. The fauna of the Bokkeveld Beds. Geological Magazine 4: 165-171, 222232. http://dx.doi.org/10.1017/S0016756800131711

Reed, F.R.C. 1925. Revision of the fauna of the Bokkeveld Beds. Annals of the South African Museum 22: 27-225.

Richter, R. 1941. Devon: Geologische Jahresberichte. Berlin 3A: 31- 43. 
Richter, R. and Richter, E. 1942. Die Trilobiten der Weismeschichten am Hoen Venn, mit Benerkungen über die malvinocaffrische Provinz. Seckenbergiana 25 (2): 156-179.

Salter, J.W. 1856. Description of Paleozoic Crustacea and Radiata from South Africa. Transactions of the Geological Society of London 2 (7): 215-224.

Salter, J.W. 1864. A monograph of the British trilobites from the Cambrian, Silurian, and Devonian formations. Monographs of the Palaeontographical Society, London, p. 1-80.

Scheffler, S. M. 2010. [Crinóides e blastóides do Devoniano brasileiro. Tese de Doutorado, Universidade Federal do Rio de Janeiro, Instituto de Geociências, Rio de Janeiro, 278 p. Unpublished.]

Scheffler, S.M.; Silva, S.D.; Gama Júnior, J.M.; Fonseca, V.M.M. and Fernandes, A.C.S. 2011. Middle Devonian crinoids from the Parnaiba Basin (Pimenteira Formation, Tocantins State, Brazil). Journal of Paleontology 85 (6): 1188-1198.

Stasi, A.M. 2013. [Revisão sistemática dos Calmoniidae (Trilobita, Phacopida) da Formação Ponta Grossa, Devoniano, Bacia do Paraná, Brasil. Dissertação de Mestrado, Universidade de São Paulo, São Paulo, 91 p. Unpublished]

Ulrich, A. 1892. Palaeozoische Versteinerungen aus Bolivien. In: G. Steinmann (Ed.), Beitriige zur Geologie und Paliontologie von Siidamerika, Schweizerbart, Stuttgart, p. 1-116.

Velazco, Y.P. 2012. Fósiles del paleozoico de la colección Antonio Raimondi conservados en el Museo de Historia Natural - Universidad Nacional Mayor de San Marcos, Lima-Perú, $1^{\circ}$ parte. Revista del Instituto de Investigación (RIIGEO), FIGMMG-UNMSM 15 (29): 7-16.

Wolfart, R. 1968. Die Trilobiten aus dem Devon Boliviens und ihre Bedeutung fir stratigraphie und tiergeographie. In: R. Wolfart and A. Voges (Eds.), Beitriige zur Kenntnis des Devons von Bolivien, Beihefte zum Geologischen Jahrbuch (74), p. 5-201. 
ANEXO IV

SYSTEMATIC REVISION AND PALEOBIOGEOGRAPHY OF CALMONIID TRILOBITES (PHACOPIDA) FROM THE DEVONIAN OF THE AMAZON BASIN, BRAZIL Artigo Será Submetido ao Periódico Geologia USP - Série Científica 
SYSTEMATIC REVISION AND PALEOBIOGEOGRAPHY OF CALMONIID TRILOBITES (PHACOPIDA) FROM THE DEVONIAN OF THE AMAZON BASIN, BRAZIL

\title{
REVISÃO SISTEMÁTICA E PALEOBIOGEOGRAFIA DE TRILOBITAS CALMONÍDEOS (PHACOPIDA) DO DEVONIANO DA BACIA DO AMAZONAS, BRASIL
}

Felipe van Enck Meira ${ }^{1}$, Juliana de Moraes Leme ${ }^{2}$

${ }^{1}$ Programa de Pós-graduação em Geoquímica e Geotectônica, Instituto de Geociências, Universidade de São Paulo - USP, São Paulo, SP, BR (fvemeira@ gmail.com)

${ }^{2}$ Departamento de Geologia Sedimentar e Ambiental, Instituto de Geociências, Universidade de São Paulo - USP, São Paulo, SP, BR (leme@usp.br)

\begin{abstract}
A systematic revision of some trilobites from the Devonian (Eifelian) of the Amazon Basin is herein presented. Results show that these trilobites, originally associated with the genera Phacops Emmrich, 1839, Dalmanites Barrande, 1852 and Homalonotus König, 1825 consist of Calmoniidae ascribed to the genera Malvinella Wolfart, 1968 and Pennaia Clarke, 1913, the latter with question. These results corroborate previous revisions that included Devonian Amazonian trilobites in Bolivian taxa. Migration routes are discussed based on the last systematic revisions of this trilobite family in Bolivia and Amazon Basin.
\end{abstract}

Keywords: Calmoniidae; Devonian; Amazon Basin; Paleobiogeography; Brazil.

Resumo Uma revisão sistemática de alguns trilobitas do Devoniano (Eifeliano) da Bacia do Amazonas é aqui apresentada. Os resultados mostram que estas trilobitas, originalmente associados com os gêneros Phacops Emmrich, 1839, Dalmanites Barrande, 1852 e Homalonotus König, 1825 consistem em Calmoniidae atribuídos aos gêneros Malvinella Wolfart, 1968 e Pennaia Clarke, 1913, este último com dúvidas. Estes resultados corroboram revisões anteriores que incluem trilobitas do Devoniano amazônico em táxons bolivianos. Rotas de migração são discutidas com base nas últimas revisões sistemáticas desta família de trilobitas na Bolívia e na Bacia do Amazonas.

Palavras-chave: Calmoniidae; Devoniano; Bacia do Amazonas; Paleobiogeografia; Brasil. 


\section{INTRODUCTION}

The first reports on Devonian fauna of Brazil date from the second half of the $19^{\text {th }}$ century, as a result of North American expeditions to the Brazilian state of Pará (Hartt and Rathbun, 1875; Clarke, 1890), where sedimentary rocks of Maecuru and Ererê formations of the Amazon Basin crop out (Fig. 6.1). The diversity of fossils in these units comprises brachiopods, crinoids, trilobites, tentaculitids, pelecypods and gastropods. Within these fossils, the brachiopods (Fonseca and Ponciano, 2011) and pelecypods (Machado, 1990) are more frequently studied; the trilobites, on the other hand, did not receive much attention throughout much of the twentieth century. More recent works have shed new light on the systematics and the paleobiogeographic relations of the trilobites of the Amazon Basin (Lieberman et al., 1991; Lieberman, 1993; Carvalho and Fonseca, 2007; Carvalho and Ponciano, 2015) but due to the fragmentary nature of these fossils, some questions are still left.

The present study analyzed the systematics of some Calmoniidae (Phacopida) trilobites originally included in the genera Homalonotus König, 1825, Phacops Emmrich, 1839 and Dalmanites Barrande, 1852 (Clarke, 1890). Results show that these trilobites consist on forms ascribed to the taxa Malvinella Wolfart, 1968, from the Devonian of Bolivia and Pennaia Clarke, 1913, from the Devonian of Bolivia, Paraná Basin, South Africa and Falkland Islands. In the latter taxon, the nomenclature was left open, as the material does not give enough information to ensure its generic status. We also discuss the paleobiogeographic implications based on the progress in the knowledge on Amazonian trilobites, especially in more recent works (Lieberman et al., 1991; Lieberman, 1993; Carvalho et al., 2003; Carvalho and Fonseca, 2007; Carvalho and Ponciano, 2015). 


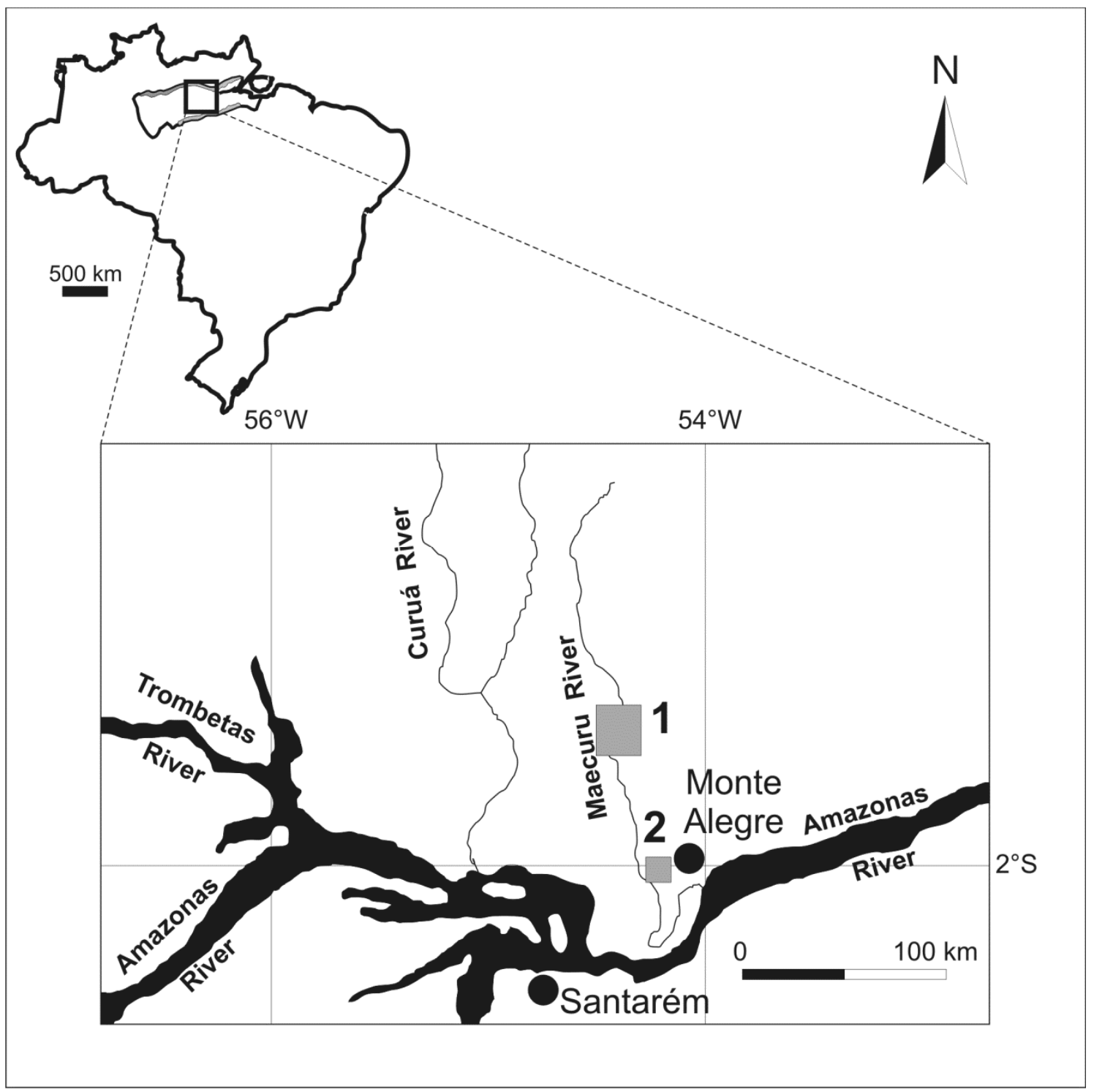

Figure 1. Location of the Devonian fossils, including trilobites, collected in the pioneering expeditions in the Amazon Basin, Pará State, northern Brazil. Areas correspond to gray rectangles. 1) Maecuru Formation fossils; 2) Ererê Formation fossils. Modified from Silva and Fonseca (2005).

\section{GEOLOGICAL SETTING}

The Amazon Basin is limited to the north by the Guyana Craton and to the south by the Brazilian Craton. Westward, the basin is separated from the Solimões Basin by the Purus Arch; eastward, the Amazon Basin is set apart from the Marajó Basin by the Gurupá Arch (Cunha et al., 2007).

The Early-Middle Devonian in the Amazon Basin is represented by the Maecuru and Ererê formations, inserted in the Urupadi Group. The type-section of this group is the interval between 
1.319 and 1.450 meters of the well 1-AM-1-AM (03' 17 'S 06" / 59 24" 52' W; Cunha et al., 2007).

The Maecuru Formation, spanning between the late Emsian and early Eifelian (Melo and Loboziak, 2003; Grahn and Melo, 2004), is mainly composed of sandstones, mudstones with rare interbedded siltstones and associated fluvial-deltaic to neritic environments (Cunha et al., 2007). This unit contains abundant fossils of marine invertebrates, including trilobites, brachiopods, crinoids, conulariids, corals, tentaculitids, bryozoans, gastropods and belerofontids (Fonseca and Ponciano, 2011) at the top of the unit (Melo, 1988).

The Ererê Formation (late Eifelian - early Givetian, Melo and Loboziak, 2003; Grahn and Melo, 2004) overlies the Maecuru Formation. In the unit predominates finer neritic sediments (fine grained sandstones, siltstones and mudstones), reflecting a transgressive event in the basin (Cunha et al., 2007) that reached its maximum just above the Eifelian/Givetian limit (Loboziak and Melo, 2003). The fossil fauna presented in the base consists of trilobites, brachiopods, pelecypods, gastropods, crinoids and tentaculitids (Melo, 1988). According to Melo (1988), though fossils in this formation are less diverse than those observed in overlying unit, its occurrence covers a wider lithological range.

\section{MATERIAL AND METHODS}

The studied material consists on external and internal molds of cranidia and pygidia, mainly from the Maecuru Formation. Specimens are housed at Instituto de Geociências of the Universidade Federal do Rio de Janeiro (IG-UFRJ) and Museu Nacional of the Universidade Federal do Rio de Janeiro (MN-UFRJ). Comparative material is housed at Museu de Ciências da Terra do Departamento Nacional de Produção Mineral (MCT-DNPM), Museo de Historia Natural Noel Kempff Mercado (MNKM), Yacimientos Petroliferos Fiscales Bolivianos (YPFB) and American Museum of Natural History (AMNH).

The systematic review of the material followed the criteria and morphological terminology used in Harrington et al. (1959), Eldredge and Braniša (1980), Lieberman et al. (1991), Lieberman (1993) and Whittington et al. (1997). For norms in open nomenclature, we followed the protocol published by Bengtson (1988). 


\section{RESULTS}

\section{Systematic Paleontology}

Order: Phacopida Salter, 1864

Family: Calmoniidae Delo, 1935

Subfamily: Calmoniinae Delo, 1935

Genus: Pennaia Clarke, 1913

Type Species: Pennaia pauliana Clarke, 1913

Pennaia? menura Clarke, 1890

Figs. 2A-B

1890. Phacops menurus Clarke, p. 17-18, pl. 1, fig. 15.

1979. Acastoides? menurus Eldredge and Ormiston, p. 157.

1982. Pennaia menurus Cooper, p. 166.

2015. Acastoides? menurus Carvalho and Ponciano, p. 41, fig. $3^{\text {a }}$

Examined material: MN 3377-I, internal mold of pygidium.

Stratigraphy and Age: Maecuru Formation (early Eifelian).

Location: Pará State, northern Brazil.

Description: Pygidium short, semicircular in outline. Length $60 \%$ of the width. Surface distinctly trilobate, with axis well elevated above the pleural field and convex. Axial furrows broad and inconspicuous, tapering abruptly on the first axial ring and ring furrow, decreasing the convergence angle behind the first ring furrow. Anteriorly, pygidial axis corresponds to about one third the pygidium maximum width. Pygidial axis with three discernible rings and possibly a fourth. Rings slightly arched forward, with constant width (sag., exsag.) through their extension. First axial ring broader (sag., exsag.) more conspicuous and more elevated. Ring furrows are broad (sag., exsag.) and shallow. Rounded axial terminus ending before the posterior margin, slightly elevated than the pleural field, with no apparent traces of rings. Pleurae sloping abruptly toward the margins. Prominent articulating half ring and three ribs observed. Ribs well 
impressed adaxially, becoming fainter close to the lateral margin. Pleural furrow narrow (exsag.), deeper adaxially, shallowing toward the lateral margin, observed only on the first rib. Anterior pleural band of the first rib more elevated and broader (exsag.). First interpleural furrow with the same width (exsag.) as the pleural furrow.

Discussion: Pennaia? menura Clarke, 1890 (Fig. 2.A-B) shows great similarity with the Pennaia species P. pauliana Clarke, 1913 from the Ponta Grossa Formation of Brazil and with $P$. verneuili D'Orbigny, 1842, from the Icla and Sica Sica formations of Bolivia (Fig. 2.C-D) in the semicircular outline of pygidium, axis well set off from pleura and composed of few rings (anterior), and rounded terminal piece. Likeness is even more remarkable with the Bolivian species, which also presents an elevated profile. Marginal lappets in the pygidium occur in number of three or four in Pennaia pauliana (Clarke, 1913), and two in P. verneuili (Reed, 1927), albeit Eldredge and Braniša (1980) reported a thoracopygidium of $P$. verneuili from the Icla Formation at Padilla with two large pair of marginal lappets plus a third minor pair. In the exemplar from the Maecuru Formation, no marginal lappets are preserved. The Brazilian sample differs from Pennaia pupillus Lake, 1904, from the Devonian Gydo Formation, Bokkeveld Group, South Africa, in having fewer rings on the axis, and the axis ending before the posterior margin. The species Pennaia gamonedensis Eldredge and Braniša, 1980, from the Gamoneda Formation of Bolivia shares with Pennaia? menura the few quantity of rings on the axis, but differs from the latter in the posterior end of axis, somewhat more acute than rounded; additionally, in P. gamonedensis the pygidium seems to be less elevated in profile. Therefore, in the present work, the Amazonian fossil is assigned with question to the genus Pennaia. Although the material is very similar to Pennaia pauliana and $P$. verneuili, its poor preservation and the absence of additional specimens avoid a more precise classification. The Amazonian fossil was previously ascribed to Acastoides Delo, 1935 with question by Eldredge and Ormiston (1979), although they did not justify the ascribing. Later, Eldredge and Braniša (1980) dissociated the Bolivian Acastoides from the Silurian-Devonian Boreal Acastidae Acastoides, positioning the former within the Calmoniidae and leaving its generic name open ("Acastoides"). Cooper (1982) endorsed Eldredge and Braniša's (1980) statement, placing all the material inserted in "Acastoides" to the genus Pennaia, including the Maecuru Formation fossil. 


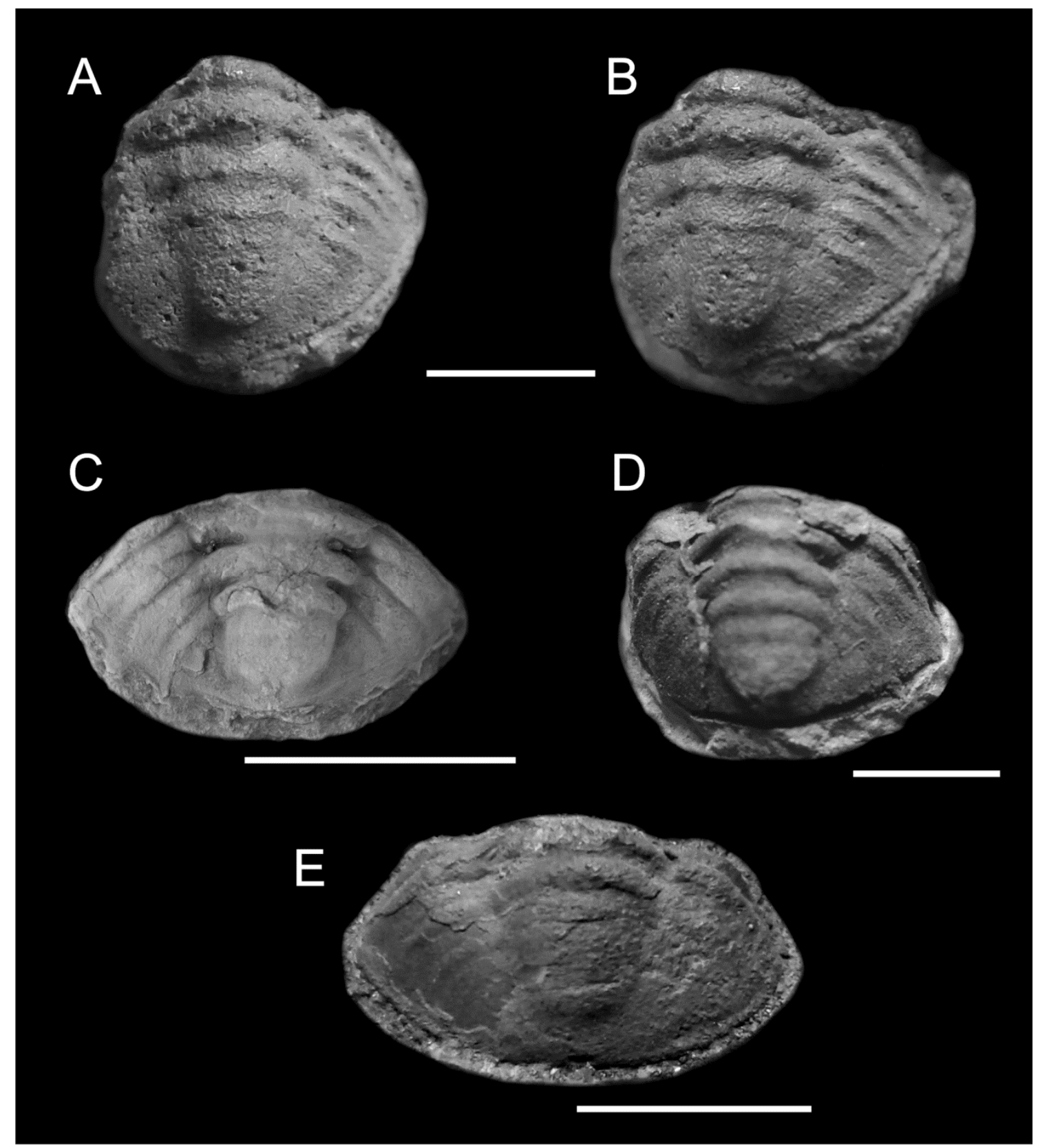

Figure 2. A-B) Pennaia? menura Clarke, 1913, Maecuru Formation, Brazil (MN 3377-I. Syntype), in dorsal (A) and lateral (B) views. C) Pennaia pauliana Clarke, 1913, Ponta Grossa Formation, Brazil (72-DGM). D) Pennaia verneuili D’Orbigny, 1842, Icla Formation, Bolivia (AMNH 78196). E) Pennaia? pullina Clarke, 1890, Maecuru Formation, Brazil (MN 3379-I. Syntype). Scale bars represent $1 \mathrm{~cm}$.

Pennaia? pullina Clarke, 1890

Fig. 2E

1890. Phacops? pullinus Clarke, p. 20-21, pl. I, fig. 12.

1925. Phacops? pullinus Reed, p. 121. 
Examined material: MN 3379-I, internal mold of pygidium.

Stratigraphy and Age: Maecuru Formation (early Eifelian).

Location: Pará State, northern Brazil.

Description: Pygidium semicircular, length (sag.) about 55\% the width. Anterior margin convex. Axial furrows shallow and inconspicuous. Three or four axial rings observed. Terminal axial piece in ogival shape, not reaching the posterior margin. Axis slightly convex above the pleural field in lateral view. First two axial rings moderately arched forward, it is not evident in the following rings. Ring furrow between first and second axial ring broad (sag., exsag.) and well-incised. Three pairs of narrow (exsag.) pleural ribs observed. Pleural ribs faintly impressed, gently curved backward and downward, not reaching the lateral margins. Interpleural furrows broad and flattened (exsag.). Pleural field behind the third pair of ribs apparently smooth.

Discussion: A systematic revision of Pennaia? pullina (Fig. 2E) is difficult because it consists of a single micropygous pygidium, devoid of spines or other ornaments that could precise its taxonomic status. Pennaia? pullina was noted by Reed (1925) to bear a resemblance with pygidia from the Bokkeveld Group initially named as Proetus hexensis by this author, but later reclassified as Phacopina hexensis by Cooper (1982). By comparing the Brazilian material with the description of the lectotype and the photograph of P. hexensis in Cooper (1982), Pennaia? pullina is quite similar to the South African fossil, however in Phacopina hexensis the pygidial axis tapers into a rather ogival acute end, in a similar manner as in Andinacaste chojnacotensis Swartz, 1925 (Wolfart, 1968, pl. 23, fig. 5). In the Amazonian sample, it tapers roundly, like that in Pennaia verneuili and Pennaia pauliana. Particularly, it is more similar to the latter species, as both bear a wider pygidium, with a flattened profile. Nevertheless, no marginal spines are present along the margins of Pennaia? pullina, despite the pygidium margins seem well preserved. This differs from the margins of Pennaia pauliana which bear three spines on each side.

Pennaia? pullina also shares similarities with Pennaia gamonedensis, as both present a rather smooth surface, few axial rings and an apparent long tapering (sag.) terminal piece and absence of ornaments or spines.

The Amazonian fossil is therefore placed with question in the genus Pennaia, due to its similarity with Pennaia species, particularly with $P$. pauliana. The scarcity and fragmentary 
nature of the Amazonian material prevent more accurate classification, and more complete specimens are required.

Genus: Malvinella Wolfart, 1968

Type Species: Anchiopella haugi Kozlowski, 1923

Malvinella acanthura Clarke, 1890

Figs. 3A-D

1890. Homalonotus (Calymene) acanthurus Clarke, p. 11-14, pl. 1, figs. 9-10.

1979. Tarijactinoides? acanthurus Eldredge and Ormiston, p. 162.

2015. Homalonotus? (Calymene) acanthurus Carvalho and Ponciano, p. 43, fig. 3F.

Material examined: MN 3374-I, internal mold of pygidium, designated as lectotype by Carvalho and Ponciano (2015); UFRJ 102-Tr, internal and external molds of pygidium (two exemplars), here designated as paralectotypes; MN 2913-I, internal mold of pygidium.

Stratigraphy and Age: Maecuru Formation (early Eifelian).

Location: Pará State, northern Brazil.

Description: Pygidium subtriangular in shape, length (sag.) about $70 \%$ the width. Anterior margin strongly convex. Axial furrows broad and shallow, gradually converging toward the posterior end. Maximum of nine axial rings observed. Triangular-tipped terminal lappet, slightly vaulted upward, embracing the last three or four rings. Axis convex above the pleural field in lateral view. First axial rings moderately curved forward, this curvature becomes less evident throughout the posterior rings. Axial rings with constant length (sag., exsag.). Ring furrows wellincised and wide (sag., exsag.). Elongated (tr.) apodemal pits observed abaxially on the first five ring furrows, close to the axial furrows. Six pairs of pleural ribs observed. Pleural ribs strongly curved backward and downward. Interpleural furrows narrow, more evident between the first and the second and the second and the third ribs. Pleural furrows narrow and well-incised. Anterior pleural band significantly more elevated than the posterior one. Ribs reaching the lateral margins, giving a crenulated pattern to them. 
Discussion: The comparison between the pygidium from the Maecuru Formation classified as Homalonotus (Calymene) acanthurus by Clarke (1890) (Fig. 3.A-D) and some pygidia of Malvinella buddeae Lieberman, Edgecombe and Eldredge, 1991 from the Belén Formation of Bolivia (Fig. 3.E-G) have shown no apparent dissimilarities, except for the absence of tubercles in the Amazonian fossils.

The inclusion with question of Malvinella acanthura in the genus Tarijactinoides SuárezSoruco, 1971 by Eldredge and Ormiston (1979) is not supported in the present work. The pleural field in Tarijactinoides is flat adjacent to the axial rings (Fig. 3.H-I) presenting a strong slope downward and backward close to the lateral margins (Fig. 3I). In the Brazilian material, the pleural field slopes downward and backward close to the axial furrows, hence no flattened area is present. Besides, Tarijactinoides' axial rings and ring furrows are sinuous, especially posteriorly (Fig. 3H). From the limits with the axial furrows, the axial rings and ring furrows curve forward adaxially, vaulting backward at the midline. In the Maecuru Formation specimen, the first two or three axial rings are slightly arched forward, and the posterior ones are straighter (Fig. 3.A-B). In a recent review, Carvalho and Ponciano (2015) noted that $M$. acanthura consists of a Calmoniidae from the Metacryphaeus Group, but rather prefer to leave the genus in open nomenclature.

Therefore, Homalonotus (Calymene) acanthurus is here inserted in the calmoniid genus Malvinella. 


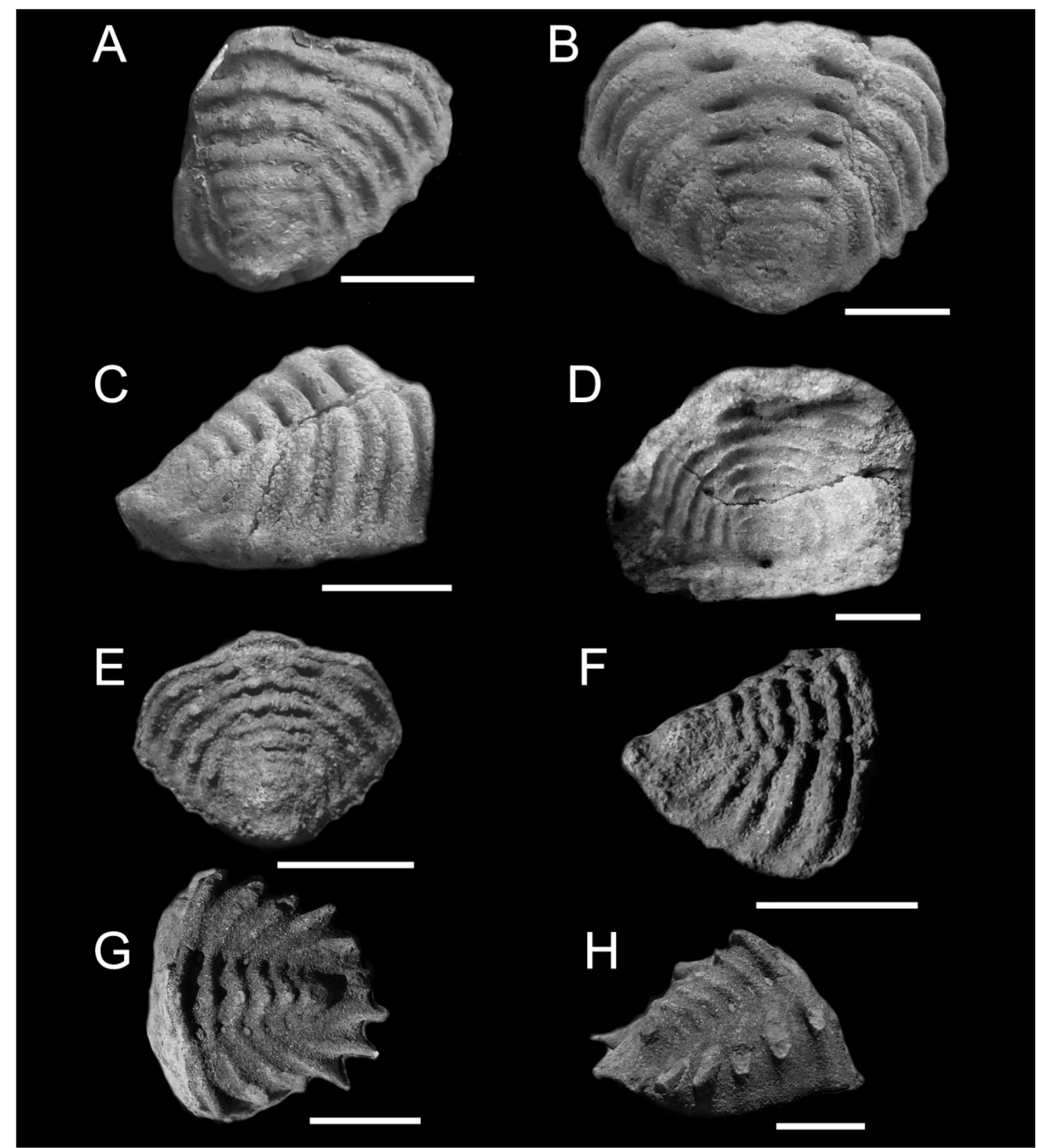

Figure 3. A-D) Malvinella acanthura Clarke, 1890, Maecuru Formation, Brazil. A) (MN 3374-I. Lectotype). B-D) (102-Tr. Paralectotypes), internal mold in dorsal (B) and lateral (C) views; D) External mold. E-G) Malvinella buddeae Lieberman et al., 1991, Belén Formation, Bolivia. EF) Sample MKMP-2863 in dorsal (E) and lateral (F) views. G) External mold (MKMP-3441). H-I) Tarijactinoides jarcasensis Suárez-Soruco, 1971, Tarija Formation, Bolivia (Pal-0269), in dorsal $(\mathrm{H})$ and lateral $(\mathrm{I})$ views.. Scale bars represent $1 \mathrm{~cm}$. 
Malvinella tumiloba Clarke, 1890

Figs. 4A-B

1890. Dalmanites tumilobus Clarke, p. Clarke, p. 35-38, pl. 2, figs. 9, 12.

1968. Malvinella? tumiloba Wolfart, p. 106, 110.

2015. Malvinella? tumiloba Carvalho and Ponciano, p. 42, fig. 3D.

Examined material: MN 3393-I, internal mold of glabella, here designated as lectotype (figured by Clarke, 1890: pl. 2, fig. 9) and figured in Fig.; MN 3391-I and UFRJ 116-Tr, internal mold of cranidium, here designated as paralectotypes; UFRJ 107-Tr, MN 2877-I, MN 3392-I (internal mold of glabella).

Stratigraphy and Age: Maecuru Formation (early Eifelian) and probably Ererê Formation (late Eifelian).

Location: Pará State, northern Brazil.

Description: Glabella subpentagonal to circular, width at frontal glabellar lobe (tr.) $85 \%$ the glabella length (sag.). Frontal glabellar lobe (LA) slightly more inflated than the lateral glabellar lobes; L0 straight, in some exemplars it bears a moderate arching backward; lateral glabellar lobe L1 narrow (exsag.), wedge-shaped, with the same length (tr.) as L2; L2 and L3 vaulted backward inflated and conspicuous, sometimes slightly more elevated than the glabellar frontal lobe. L2 and L3 abaxially wide (exsag.), becoming narrower toward the median area, L3 wider than L2 close to the axial furrows, with a sharper tapering adaxially. Frontal glabellar lobe rhomboid, laterally expanded, anterior margin gently convex. Preglabellar area wide (tr.), shorter (exsag.) abaxially becoming longer (sag.) at the median area. S0 broad (sag., exsag.) and conspicuous, strongly convex forward medially on the anterior edge, with elongated apodemal pits abaxially, close to the axial furrows. S1 narrow (exsag.), strongly curved backward, in contact with axial furrows. S2 shallow and straight, less conspicuous than S1, contact with axial furrows not clear. S3 broad, well-impressed, obliquely curving toward the median area, reaching the axial furrows.

Discussion: Wolfart (1968) assigned Dalmanites tumilobus Clarke, 1890 (Fig. 4.A-B) with question to the genus Malvinella Wolfart, 1968, due to the scarceness of data in the Brazilian taxon, including absence of pygidium and even part of cephalon. 
In the present work we endorse Wolfart's (1968) suggestion in including Dalmanites tumilobus in the genus Malvinella and rectify its generic status. According to Wolfart (1968), Malvinella tumiloba differs from Bolivian Malvinella in the shapes of S1 and S2 glabellar furrows. In our observations we did not find any differences between the glabellar furrows in the Amazonian material and the species Malvinella haugi Kozlowski, 1923 and Malvinella buddeae from the Belén Formation of Bolivia. The similarities are more evident between M. tumiloba and $M$. buddeae (Fig. 4.C-D), especially the L2 and L3 morphology and the absence of a bulged glabella across S2-L3, this latter a diagnostic character exclusive of M. haugi (Lieberman et al., 1991), but curiously also found in Malvinella australis Clarke, 1890 (Fig. 4.E), an Amazonian taxon from the Maecuru Formation recently revised by Carvalho and Ponciano (2015). Wolfart (1968) also alleged that the smooth glabella in Malvinella tumiloba contrasts with the tuberculate surface in the Bolivian Malvinella. This feature may have been produced by the effacement caused by weathering. This condition is quite frequent in trilobites of the Ponta Grossa Formation, Devonian of the Paraná Basin (Soares et al., 2008). Wolfart (1968) pointed that the preglabellar area in Malvinella tumiloba is broader (tr.) and shorter (sag.) than that in Bolivian Malvinella. Indeed, in specimens of Malvinella tumiloba where the preglabellar area is present (Fig. 4.A-B) it is different from what is seen in Bolivian Malvinella. In the latter, the glabellar frontal lobe protrudes the anterior border, and a narrow (tr.) bluntly pointed anterior median process is observed (Fig. 4D). However, apart from the difference in median process morphology, Malvinella tumiloba shares all other features with representatives of the genus Malvinella and therefore is here placed within this genus.

We report the occurrence of a fragmented glabella (Fig. 4F) collected by the Comissão Geológica do Império at the Monte Alegre municipality (Fig. 1B), in the Pará State, here ascribed to Malvinella cf. tumiloba. Fossils collected in this area are attributed to the Ererê Formation, probably from the type locality of this unit (Oliveira and Leonardos, 1978). Therefore, the trilobite Eldredgeia paituna Hartt and Rathbun, 1875 is not the only calmoniid from the Ererê Formation. 


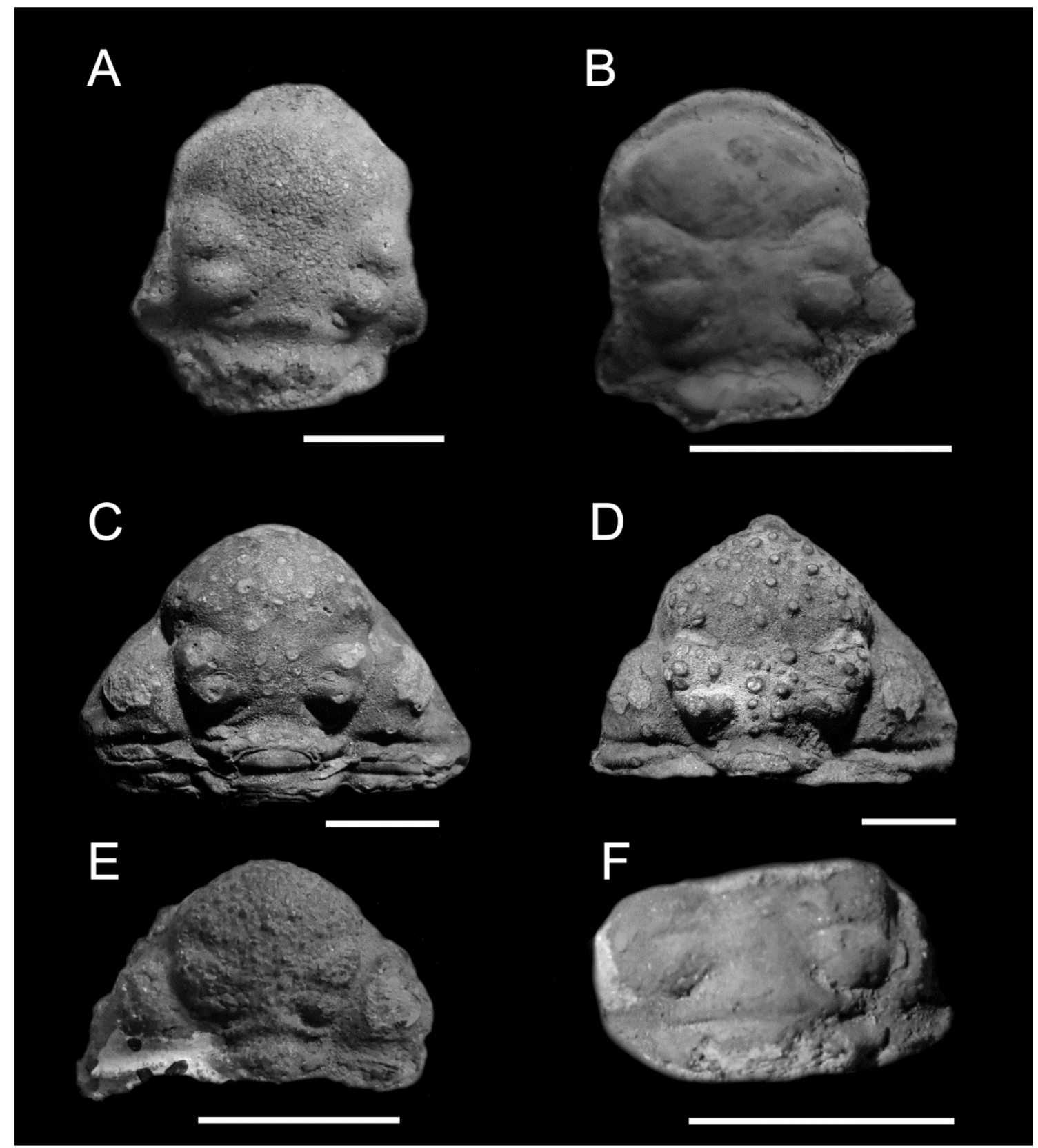

Figure 4. A-B) Malvinella tumiloba Clarke, 1890, Maecuru Formation, Brazil. A) (MN 3393-I. Lectotype). B) (116-Tr. Sintype). C) Malvinella buddeae Kozlowski, 1923, Icla Formation, Bolivia (MKMP 3967). D) Malvinella buddeae Lieberman et al., 1991, Belén Formation, Bolivia (MKMP-996). E) Malvinella australis Clarke, 1890, Maecuru Formation, Brazil (MN 3389-I). F) Malvinella cf. tumiloba, Ererê Formation, Brazil (MN 2877-I). Scale bars represent $1 \mathrm{~cm}$. 


\section{CALMONIID PALEOBIOGEOGRAPHY IN THE AMAZONAS BASIN}

Although the Amazon and Parnaíba basins present the same tectono-sedimentary evolution observed in other Brazilian intracratonic basins, as the Paraná and Parecis, their Devonian faunal composition is different from the these latter localities, with most of brachiopod taxa allied to elements of the Eastern Americas Realm, with minor influence of the Old World Realm (presence of the brachiopod Tropidoleptus carinata Conrad, 1839 in both basins) (Melo, 1988). The trilobites, on the other hand, show Malvinokaffric affinities, especially with Bolivian forms (Eldredge and Ormiston, 1979). This led to different opinions between authors. Boucot (1988) grouped the Amazon and Parnaíba basins in a Eastern Americas province, denominated Amazon Sub-province. Contrarily, Eldredge and Ormiston (1979) concluded that the calmoniids were endemic to a province named by them Malvinokaffric Realm, inserting in it all the localities where calmoniids occur, including the Amazon and Parnaíba basins. However, the recent discover of calmoniids in the late Emsian lower part of the Floresta Formation in Colombia (Morzadec et al., 2015), an Eastern Americas locality from the Colombian Sub-province (Boucot, 1988), challenges Eldredge and Ormiston's (1979) statement. In more recent works, the Amazon and Parnaíba basins are treated as paleobiogeographic boundaries between the Eastern Americas and the Malvinokaffric realms, due to the mixing character of the marine faunas mainly from these areas (Melo, 1988; Carvalho and Fonseca, 2007).

\section{Migration routes to the Amazon Basin}

Besides calmoniids, the Devonian trilobite fauna of the Amazon Basin is composed of the homalonotids Digonus derbyi Clarke, 1890 and Burmeisteria oiara Hartt and Rathbun, 1875 (Carvalho and Ponciano, 2015), the synphoriid Amazonaspis maecurua Clarke, 1890 (Carvalho and Fonseca, 2007), and dalmanitids with open nomenclature (Carvalho and Ponciano, 2015). According to Carvalho and Ponciano (2015), Digonus has closest relatives in the Old World Realm and may have reached the Amazon Basin through a seaway northeast of the Amazon Basin, during the Eifelian transgressive event. The synphoriid Amazonaspis maecurua, on the other hand, is related to Eastern Americas forms, and may have migrated to the Amazon Basin via a seaway southwest the basin, after reaching the north of South America (Colombian Subprovince) (Carvalho and Fonseca, 2007, Fig.4). 
Most calmoniid representants in the Amazon basin are nested within the Malvinella Sub-group of the Metacryphaeus Group (Lieberman et al., 1991), belonging to the Malvinella genus. Eldredgeia paituna Hartt and Rathbun, 1875 from the Ererê Formation (Carvalho et al., 2003) also belongs to the Metacryphaeus Group, albeit not included in the Malvinella Sub-group (Lieberman, 1993).

Except for the taxa whose genera remained open (e.g. Palpebrops? goeldii Katzer, 1903, Carvalho and Ponciano, 2015), four genera of calmoniids are known to occur in the Amazonas Basin - Vogesina Wolfart, 1968, Phacopina Clarke, 1913, Malvinella and Eldredgeia Lieberman, 1993. They consist on the species Vogesina gemellus Clarke, 1890, Malvinella australis Clarke, 1890 (Lieberman et al., 1991; Carvalho and Ponciano, 2015), Malvinella tumiloba, Malvinella acanthura (present work), Eldredgeia paituna Hartt and Rathbun, 1875 (Lieberman, 1993; Carvalho et al., 2003; Carvalho and Ponciano, 2015) and Phacopina braziliensis Clarke, 1890. Most of species are present in the Maecuru Formation; the Ererê Formation only bears Eldredgeia paituna and Malvinella cf. tumiloba.

As in the case of synphoriids (Carvalho and Fonseca, 2007), Melo (1988) suggested that the Bolivian-related calmoniids of the Maecuru Formation migrated to the Amazon Basin through this same seaway to the southwest during the Eifelian transgressive event that set up during the deposition of the Upper Maecuru Formation and most of the overlying Ererê Formation (Fig. 5). This seaway as a route for Bolivian calmoniids seems more parsimonious considering the absence of these genera in both the Paraná and Parnaíba basins, an optional route along which these could have crossed to reach the Amazonas Basin (Fig. 5A). Therefore, the early Eifelian calmoniids from the Maecuru Formation might have derived from Bolivian forms of Emsian age from the Belén and Icla formations of Bolivia that migrated to the Amazonas Basin through this seaway westward of the basin.

For the late Eifelian Ererê Formation, Melo (1988) inferred that Eldredgeia Lieberman, 1993 (= Metacryphaeus Reed, 1907) may have reached the Amazon Basin via Parnaíba Basin, where closely related or identical forms are present. Melo's (1988) supposition was based on the species Metacryphaeus meloi Carvalho, Edgecombe and Lieberman, 1997, from the early Givetian Passagem Member of the Pimenteira Formation, which is similar in overall aspect to the Amazonian E. paituna. Nevertheless, Lieberman et al. (1991) described Eldredgeia cf. venusta from the late Eifelian Pimenteira Formation near Picos city, eastern flank of the Parnaíba Basin. According to Lieberman et al. (1991), the difference between Pimenteira Eldredgeia and Bolivian Eldredgeia venusta Wolfart, 1968, is slight, with the former having the L0 longer relative to its width and S1 more sharply curved forward proximally, the latter condition also 
present in some specimens of Eldredgeia cf. venusta from the late Eifelian Waboomberg Formation of the Bokkeveld Group, South Africa (Cooper, 1982).

Given the occurrence of Eldredgeia in the Parnaíba Basin, it is inferred that the migration route of this taxon from Bolivia - where the older occurrences of Eldredgeia venusta date from the Emsian of the Belén and Icla formations - to the Amazonas Basin, happened through a connection between Bolivia - Alto Garças Sub-basin - Parnaíba Basin - Amazonas Basin (Fig. 5B). In this scenario, a population of originally Bolivian E. venusta reached the Parnaíba Basin through Alto Garças Sub-basin at the Eifelian; it probably did not happened prior to the Middle Devonian as chitinozoan species from the Paraná Basin are not known in the Parnaíba and Amazonas basins before the Eifelian (Lange, 1967). Speciation then may occurred in the Amazon Basin with Eldredgeia paituna.

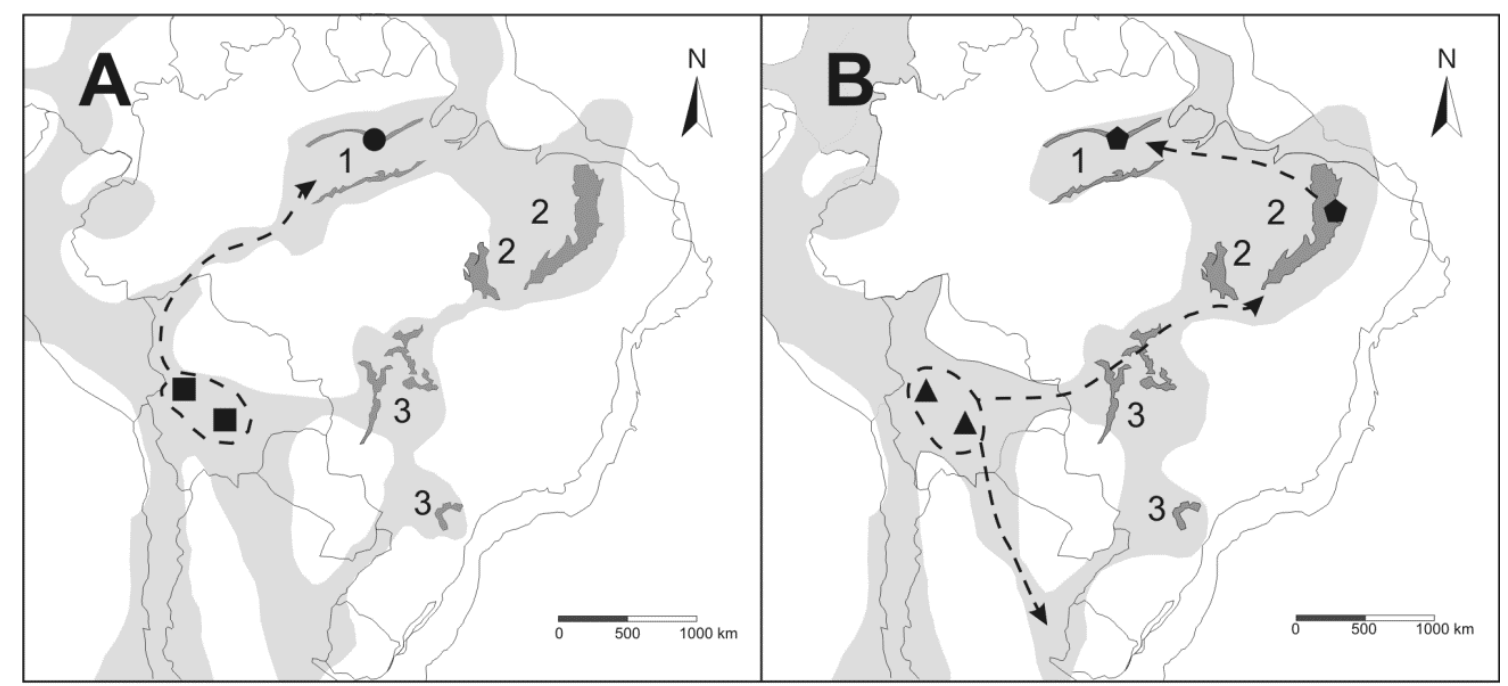

Figure 5. Paleogeographic reconstructions for the late Emsian-Eifelian (A) and late EifelianGivetian of Brazil and adjacent areas, showing the outcrop belts from the three Brazilian main paleozoic sedimentary basins: Amazon Basin (1), Parnaíba Basin (2) and Paraná Basin (3). Dashed arrows are inferred migration routes of calmoniid trilobites. Black squares and circles on A represent respectively Emsian and early Eifelian calmoniid genera (Vogesina, Phacopina and Malvinella). On B, black triangles are Emsian occurrences of Eldredgeia venusta, while pentagons represent Eldredgeia from late Eifelian-Givetian of Parnaíba and Amazon basins. Modified from Melo (1988). 


\section{Diversity in the Amazon Basin}

The Maecuru Formation is known to bear greater diversity of invertebrate fossils when compared to the overlain Ererê Formation. A total of ten calmoniids (including species with open nomenclature), two dalmanitids and a homalonotid are known from this unit, while only two calmoniids (Eldredgeia paituna and Malvinella cf. tumiloba) and a homalonotid occur in the Ererê Formation.

Nevertheless, most of the trilobite fossils from these units were named after disarticulated parts of the animals (e.g. cranidia, pygidia). Sometimes, a species is known only from a single disarticulated specimen. Given the fragmentary nature of these fossils, we do not rule out the possibility that some of them correspond to distinct parts of a same species. Hence, the diversity of trilobites especially in the Maecuru Formation may be narrower than supposed.

Based on the subjects exposed above, new investigations to the Devonian of the Amazon Basin are fundamental to get more accurate information on this still poorly understood invertebrate fossil group at this locality. Moreover, considering that, in the case of Maecuru Formation fossils, these disarticulated exoskeletons deposited in shallow marine conditions (Carvalho and Ponciano, 2015), efforts to locate taphofacies where these fossils occur as articulating assemblages would be crucial to enhance the knowledge on systematics of this group in the Amazon Basin.

\section{CONCLUSIONS}

Malvinella is the commonest calmoniid genus in the Amazon Basin, with three species ( $M$. australis, M. acanthura and M. tumiloba). This genus, along with Vogesina and Phacopina, may have reached the Amazonas Basin by the Eifelian through a seaway linking Bolivia to this basin westwards. The absence of these genera in adjacent basins supports this hypothesis;

The genus Eldredgeia, present in the late Eifelian Ererê Formation, may have reached the Amazonas Basin via Bolivia-Alto Garças Subbasin-Parnaíba Basin, as it occurs in the Pimenteira Formation of the Parnaíba Basin; 
The Amazon Basin might represent an endemism area, as it yields species not known to other localities (e.g. Digonus derbyi, Amazonaspis maecurua), but among calmoniids the diversity may be not as great as supposed, as almost all representants are based on fragmentary material (cranidia, pygidia) that may correspond to fewer species;

New field works to the Amazon Basin, especially to search for autochthonous assemblages, is crucial to verify the diversity of calmoniids and to better understand their systematics, as the trilobite material collected to date represent fragmentary parautochthonous assemblages from shallow water.

\section{ACKNOWLEDGEMENTS}

F. van E. Meira thanks the Fundação de Amparo à Pesquisa do Estado de São Paulo (FAPESP) for financial support (Process number 2012/07075-3). The authors are thankful to Bushra Hussaini (AMNH), Mario Suárez-Riglos (MNKM, YPFB), Ismar de Souza Carvalho, Flávia Alessandra Figueiredo and Mônica de Medina Coeli (IG-UFRJ), Antonio Carlos Sequeira Fernandes, Marcia Fernandes de Aquino Santos and Vera Medina da Fonseca (MN-UFRJ), Rodrigo da Rocha Machado, Irma Tie Yamamoto and Rita de Cassia Tardin Cassab (MCTDNPM) for allowing the visitation to the collections and for helping with the location of the material. F. van E. Meira also thanks Angela Meira Faleiros for article revision and further discussions.

\section{REFERENCES}

Bengtson, P. (1988). Open nomenclature. Palaeontology, 31, 223-227.

Boucot, A.J. (1988). Devonian biogeography: na update. In: N.J. McMillan, A.F. Embry, O.J. Glass (Eds.). Devonian of the world (v.1, 211-227). Calgary: Canadian Society of Petroleum Geologists. 
Carvalho, M.G.P., Edgecombe, G.D., Lieberman, B.S. (1997). Devonian calmoniid trilobites from the Parnaíba Basin, Piauí State, Brazil. American Museum Novitates, 3192, 1-11.

Carvalho, M.G.P., Edgecombe, G.D., Smith, L. (2003). New calmoniid trilobites (Phacopina, Acastoidea) from the Devonian of Bolivia. American Museum Novitates, 3407, 1-17.

Carvalho, M.G.P., Fonseca, V.M. (2007). "Dalmanites" maecurua Clarke, 1890 (Middle Devonian, Amazon Basin, Brazil) and the new genus Amazonaspis (Synphoriidae), American Museum Novitates, 3591, 1-14.

Carvalho, M.G.P., Ponciano, L.C.M.O (2015). Devonian trilobites from the Amazonas Basin: systematic diversity, taphonomy and biogeographic significance. Boletim do Museu Paraense Emílio Goeldi. Ciências Naturais, 10 (1), 37-48.

Clarke, J.M. (1890). As trilobitas do Grez de Ererê e Maecuru, Estado do Pará, Brazil. Archivos do Museu Nacional, 9, 1-58.

Clarke, J.M. (1913). Fósseis devonianos do Paraná. Monografia do Serviço Geológico e Mineralógico do Brasil, 1, 1-353.

Cooper, M.R. (1982). A revision of the Devonian (Emsian-Eifelian) Trilobita from the Bokkeveld Group of South Africa. Annals of The South African Museum, 89, 1-174.

Cunha, P.R.C., Melo, J.H.G., Silva, O.B. (2007). Bacia do Amazonas. Boletim de Geociências da Petrobras, 15 (2), 227-251.

Eldredge, N., Ormiston, A.R. (1979). Biogeography of Silurian and Devonian Trilobites of the Malvinokaffric Realm. In: A.J. Boucot, J. Gray (Eds.). Historical Biogeography, Plate Tectonics, and Changing Environment (147-167). Corvalis: Oregon State Univ. Press. 
Eldredge, N., Braniša, L. (1980). Calmoniid trilobites of the Lower Devonian Scaphiocoelia Zone of Bolivia, with remarks on related species. Bulletin of the American Museum of Natural History, 165 (2), 181-289.

Fonseca, V.M.M., Ponciano, L.C.M.O. (2011). Braquiópodes do Devoniano Médio das bacias do Amazonas e Parnaíba. In: I.S. Carvalho, N.K. Srivastava, O. Strohschoen Jr., C.C. Lana (Orgs.). Paleontologia: cenários de vida. (v. 4, 169-190). Rio de Janeiro: Interciência.

Grahn, Y., Melo, J.H.G. (2004). Integrated Middle Devonian chitinozoan and miospore zonation of the Amazonas Basin, northern Brazil. Revue de Micropaléontologie, 47, 71-85.

Harrington, H.J., Henningsmoen, G., Howell, B.F., Jaanusson, V., Lochman-Balk, C., Moore, R.C., Poulsen, C., Rasetti, F., Richter, E., Schmidt, H., Sdzuy, K., Struve, W., Størmer, L., Stubblefield, C.J., Tripp, R., Weller, J.M., Whittington, H.B. (1959). Trilobita. In: Moore, R. C. (Ed.). Treatise on Invertebrate Paleontology Part O Arthropoda (O38-O526). Lawrence: Geological Society of America and University of Kansas Press.

Hartt, C. F., Rathbun, R. (1875). Devonian trilobites and mollusks of Ererê, Province of Pará, Brazil. Annals Lyceum Natural History, 11, 111-127.

Lange, F.W. (1967). Biostratigraphic subdivision and correlation of the Devonian in the Paraná Basin. In: J.J. Bigarella (Ed.), Problems in Devonian Brazilian geology. (v. 21/22, 63-98) Curitiba: Boletim Paranaense de Geociências.

Lieberman, B. S. (1993). Systematics and biogeography of the 'Metacryphaeus group' Calmoniidae (Trilobita, Devonian), with comments on adaptative radiations and the geological history of the Malvinokaffric Realm. Journal of Paleontology, 67, 549-570.

Lieberman, B. S., Edgecombe, G. D., Eldredge, N. (1991). Systematics and biogeography of the "Malvinella group", Calmoniidae (Trilobita,Devonian): Journal of Paleontology, 65(5), 824-843. 
Machado, D.M.C. (1990). Biválvios devonianos da Bacia do Amazonas (formações Maecuru e Ererê): Considerações sistemáticas e paleoautoecológicas. Dissertação (Mestrado). Rio de Janeiro: Instituto de Geociências - UFRJ.

Melo, J.H.G. (1988). The Malvinokaffric realm in the Devonian of Brazil. In: N.J. McMillan, A.F. Embry, O.J. Glass (Eds.). Devonian of the world (v.1, 667-703). Calgary: Canadian Society of Petroleum Geologists.

Melo, J.H.G., Loboziak, S. (2003). Devonian - Early Carboniferous miospore biostratigraphy of the Amazon Basin, Northern Brazil. Review of Paleobotany and Palynology, 124 (3-4), 131 202.

Morzadec, P., Mergl, M., Villarroel, C., Janvier, P., Racheboeuf, P. (2015). Trilobites and inarticulate brachiopods from the Devonian Floresta Formation of Colombia: a review. Bulletin of Geosciences, 90 (2), 331-358.

Oliveira, A.I., Leonardos, O.H. (1978). Geologia do Brasil ( $3^{\text {rd }}$ Ed.). Mossoró, Escola Superior de Agricultura de Mossoró.

Reed, F.R.C. (1925). Revision of the Fauna of the Bokkeveld beds. Annals of the South African Museum, 22, 27-225.

Reed, F.R.C. (1927). Recent work on Phacopidae. Geological Magazine, 64 (8), 337-353.

Silva, C.F., Fonseca, V.M.M. (2005). Hábitos de vida dos trilobitas das formações Maecuru e Ererê, Devoniano da Bacia do Amazonas, Brasil. Revista Brasileira de Paleontologia, 8 (1), 73-82.

Soares, S.P., Simões, M.G., Leme, J.M. (2008). O papel da fossilização e do intemperismo na sistemática dos trilobites Phacopida (Calmoniidae e Homalonotidae), Formação Ponta 
Grossa, (Devoniano), Bacia do Paraná, Brasil. Revista Brasileira de Paleontologia, 11, 117128.

Whittingon, H.B., Chatterton, B.D.E., Speyer, S.E., Fortey, R.A., Owens, R.M., Chang, W.T., Dean, W.T., Jell, P.A., Lawrie, J., Palmer, A.R., Repina, L.N., Rushton, A.W.A., Shergold, J.H., Clarkson, E.N.K., Wilmot, N.V., Kelly, S.R.A. (1997). Treatise on Invertebrate Paleontology, Pt. O, Arthropoda 1, Trilobita. Revised edition. Lawrence: Geological Society of America and the University of Kansas Press.

Wolfart, R. (1968). Die Trilobiten aus dem Devon Boliviens und ihre Bedeutung für Stratigrtaphie und Tiergeographie. In: R. Wolfart, A. Voges. Beiträge zur Kenntnis des Devons von Bolivien. Beihefte zum Geologischen Jahrbuch, 74, 5-201. 
ANEXO V

THE GENUS BURMEISTERIA SALTER, 1865 (TRILOBITA, HOMALONOTIDAE) IN THE DEVONIAN OF THE PARNAÍBA BASIN, BRAZIL (Em Preparação) 


\title{
THE GENUS BURMEISTERIA SALTER, 1865 (TRILOBITA, HOMALONOTIDAE) IN THE DEVONIAN OF THE PARNAÍBA BASIN, BRAZIL.
}

\author{
F. van E. Meira ${ }^{1 *}$ J. de M. Leme
}

1 Programa de Pós-graduação em Geoquímica e Geotectônica, Instituto de Geociências, Universidade de São Paulo - USP. Rua do Lago, 562, CEP 05508-080, São Paulo/SP. Brasil.

${ }^{2}$ Departamento de Geologia Sedimentar e Ambiental, Instituto de Geociências, Universidade de São Paulo - USP. Rua do Lago, 562, CEP 05508-080, São Paulo/SP. Brasil.

*Corresponding author: fvemeira@gmail.com

\begin{abstract}
The knowledge on homalonotid trilobites in Brazil is comparatively poor when compared to other marine invertebrate fossils. The best documented genus of this trilobite family is Burmeisteria Salter, 1856, from the Early Devonian of the Paraná Basin and Middle Devonian of the Parnaíba Basin, where the species B. notica Clarke, 1913 was known as the only representative for almost a century since the classical monograph of Clarke (1913). Recent works focusing on the revision of invertebrate fossils under the perspective of Taphonomy (Simões et al., 2009), have concluded that, at least in the Paraná Basin, the genus Burmeisteria was also represented by a second species, B. herscheli Murchison, 1839. The present study makes some reflections on the current systematics of Burmeisteria species in the Brazilian basins based on the examination of homalonotid fossils from the Pimenteira Formation in the Parnaíba Basin, Brazil.
\end{abstract}




\section{INTRODUCTION}

Homalonotid trilobites are among the least studied marine invertebrate fossils in Brazil, especially when compared to other trilobite families as the calmoniids, whose studies are more abundant in the literature (e.g. Lieberman et al., 1991; Lieberman, 1993; Popp et al., 1996; Carvalho et al.,1997; Soares et al., 2008; Meira et al., in press). Within the homalonotids the commonest and best documented species is Burmeisteria notica Clarke, 1913, present in the Praguian/Emsian (Lower Devonian) Ponta Grossa Formation in the Apucarana Subbasin, Paraná Basin (Clarke, 1913) and in the Eifelian (Middle Devonian) Pimenteira Formation, in the Parnaíba Basin (Fig. 1A) (Castro, 1968).

Until recently $B$. notica was considered the only Burmeisteria species in Brazil. Simões et al. (2009) concluded that some specimens of Burmeisteria from the Apucarana Subbasin, including type specimens illustrated in Clarke (1913: 358, pl. 2, figs. 2-3), are better ascribed to the species Burmeisteria herscheli Murchison, 1839, due to the morphology of the cephalon (Fig. 1B-C). Besides, these authors accomplished that some $B$. notica fossils consist of taphotaxa of $B$. herscheli. Exoskeleton ornaments and glabellar features can be obliterated/modified via diagenesis and weathering (Fig. 1B-E) (Simões et al., 2009).

The present study makes some reflections on the current systematics of Burmeisteria species in the Brazilian basins based on the examination of homalonotid fossils from the Pimenteira Formation in the Parnaíba Basin, Brazil. 


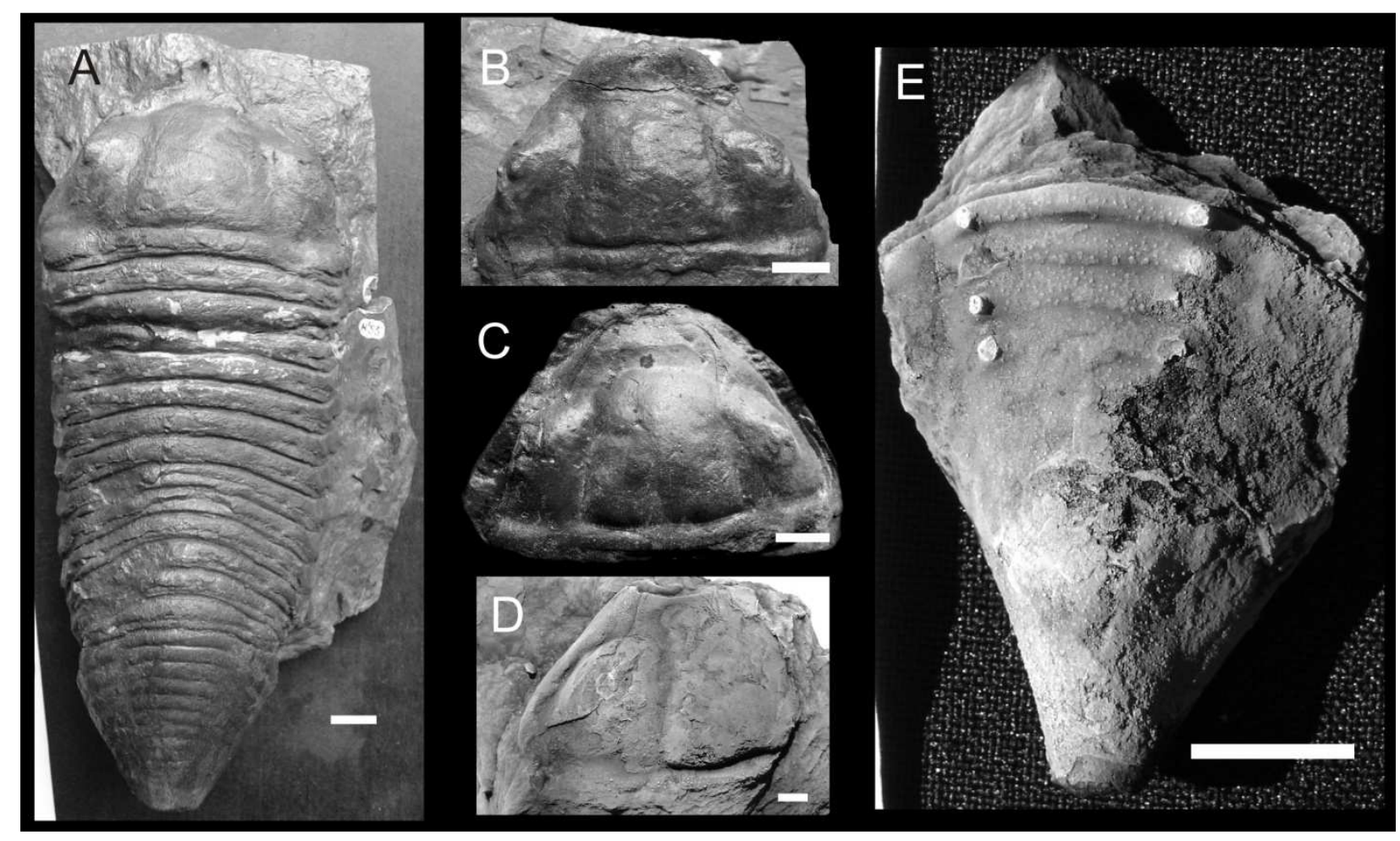

Figure 1: Burmeisteria fossils from Ponta Grossa Formation, Paraná Basin. A) 55-DGM, lectotype of B. notica Clarke, 1913 (pl. 1, figs. 1-2 in Clarke, 1913); B-C) 63-DGM and 57DGM, cephala of $B$. herscheli Murchison, 1839 (pl. 2, figs. 2-3 in Clarke, 1913); D) DZP17330, cephalon of $B$. herscheli (fig. 4A in Simões et al., 2009); E) DZP-2247b, pygidium of $B$. herscheli (fig. 4G-H in Simões et al., 2009). Scale bars correspond to $1 \mathrm{~cm}$.

\section{GEOLOGICAL SETTING}

The Devonian of the Parnaíba Basin is comprised by the Itaim, Pimenteira, Cabeças and the base of Longá formations (Vaz et al., 2007). Devonian invertebrate fossils, however, are comprised to the Middle-Upper Devonian interval, in the Pimenteira and basal Longá formations.The Pimenteira Formation, of late Eifelian/early Givetian age on the eastern basin border (Grahn et al., 2006, 2008), contains the greater variety and abundance of invertebrate fossils, including brachiopods, pelecypods, gastropods, tentaculitids, trilobites, crinoids, conularids, ostracods and hyolithids. The formation also contains vertebrates (chondrichthyans and acanthodians), and plants (Kegel 1953, Fonseca and Melo, 1987; Melo, 1985; Carvalho, 1995; Caputo et al., 2005). The early Givetian Passagem Member, formely ascribed to the Cabeças Formation (Plummer, 
1948), was recently incorporated to the top of the Pimenteira Formation, corresponding to a sandier lateral extension of this unit (Ponciano et al., 2012).

\section{MATERIAL AND METHODS}

A total of 55 trilobite specimens were examined for this study. The focus of the study were homalonotids from the Pimenteira Formation, in the areas of Picos and Pimenteiras cities, eastern Piauí State. Fossils from the village of São João Vermelho, in the southeast Piauí State, were also studied, as this locality prodided well preserved material (Leme et al., 2013). Aditionally, homalonotids from the Ponta Grossa Formation of the Apucarana Subbasin and from the Fox Bay Formation of the Falkland Islands were examined for comparison. Homalonotids from the Ponta Grossa Formation are housed at the Universidade Estadual de Ponta Grossa (UEPG), Universidade Federal do Paraná (UFPR), Universidade de Guarulhos (UnG), Museu de Ciências da Terra do Departamento Nacional de Produção Mineral (MCTDNPM) and Instituto de Geociências of the Universidade Federal do Rio de Janeiro (IG-UFRJ); homalonotids from the Pimenteira Formation are deposited in the MCT-DNPM and IG-UFRJ, and at the Fundação Homem Americano (FUMDHAM). Fox Bay Formation trilobites are housed at the IG-UFRJ and at the American Museum of Natural History (AMNH).

Some specimens from the Pimenteira Formation were coated with magnesium oxide prior to photographs in order to highlight anatomical details.

\section{RESULTS}

\section{SYSTEMATIC PALEONTOLOGY}

Order PHACOPIDA Salter, 1864

Family HomalonOTIDAE Chapman, 1890

Genus Burmeisteria Salter, 1865

Type species. Burmeisteria hersheli Murchison, 1839

Burmeisteria herscheli Murchison, 1839 
Description (after Simões et al., 2009): Burmeisteria with subtriangular cephalon; length 0.60.7 times width, with rounded genal angle. Medially constricted, urceolate glabella; length 0.81.2 times width. Tumid triangular genae, convex downward, with small eyes. Deep occipital ring furrow. Long, convex, triangular, sharply pointed pygidium; length 1.0-1.1 times width. Axis is wide, comprising 5 to 12 segments. Large, coarse structures (tubercles) present on the distal extremities of the anterior four pygidial axial rings.

Burmeisteria notica Clarke, 1913

Description (after Simões et al., 2009): Burmeisteria with subtriangular cephalon, with wide base; rounded genal angles $\left(30^{\circ}-40^{\circ}\right)$; length $0.42-0.45$ times width. Subtrapezoidal glabella, with well-marked shallow axial furrows; lobation absent; length 0.7-1.2 times glabellar width. Mammiform and conspicuous eye lobes, with prominent small eyes. Broad anterior border, terminating in the front part in a transverse margin. Very broad doublure in the front part, making a subtriangular plate, extending within the anterior edge of the glabella, narrowing at the sides, just in front of the eyes, into lateral bands continuous to the occipital ring, forming a shovel-like device. Axial and pleural segmentation distinct; axis with 9 to 13 posterior smooth rings, and 7 to 9 pairs of pleures. Transversally elongated, triangular pygidium; length $0.8-1.0$ times width. Tubercles or the base of spines not observed.

\section{DISCUSSION}

\section{Distinctiveness between $B$. notica and $B$. herscheli}

The genus Burmeisteria used to be subdivided into two subgenera: the former, B. (Burmeisteria), which included $B$. herscheli, was identified by a lobated urceolate (medially biconcave) glabella, a sigmoidal rostral suture composed of two biconcave halves meeting medially and exoskeleton covered by ornaments (tubercles or base spines); the second, B. (Digonus), included B. notica, was characterized by a smooth trapezoidal glabella and straight or slightly concave rostral suture (Harrington et al., 1959). The morphology of rostral suture as a taxonomic feature was considered of little importance by Sdzuy (1957) and Saul (1965). Cooper (1982), who had access 
to a large collection of B. herscheli from the Emsian/Eifelian Bokkeveld Group in South Africa, confirmed Sdzuy's (1957) and Saul's (1965) conclusions, suggesting that the rostral suture morphology would be rather related to developmental variations. Cooper (1982: 17) also noted every transition in glabella morphology between the two subgenera among South African Burmeisteria. The author thus concluded that the glabella shape alone could not be used to distinguish both subgenera. According to Cooper (1982), B. notica differs from B. herscheli in the complete absence of tubercles or base spines, in having a broader (sag.) preglabellar field (around 25\% the entire glabella length, whereas in B. herscheli the percentage is around 17-20\%) and in the fewer axial rings (13-15) in the pygidium (the author counted 16-17 axial rings in large $B$. herscheli individuals).

Simões et al. (2009) used the glabella morphology and the presence/absence of ornaments to differentiate the Apucarana Subbasin Burmeisteria material. According to these authors, an originally urceolate glabella, typical of $B$. herscheli would change, through compactation, to a trapezoid one, characteristic to B. notica. Glabellar lobation, present in $B$. herscheli, would be effaced through weathering processes, to a smooth glabellar surface typical of B. notica (Simões et al., 2009). Likewise, weathering processes would be responsible for obliterating ornaments on the exoskeleton. Besides this, Simões et al. (2009) reported the presence of tubercles in two pygidia, one of which with very conspicuous ornaments (Fig. 1E). As mentioned above, this character is absent in B. notica, and therefore these pygidia might belong to $B$. herscheli.

\section{Burmeisteria from Pimenteira Formation}

The study analyzed mostly specimens preserved as concretions in fine sediments of the Pimenteira Formation. One specimen of Burmeisteria notica from the sandstones of the Passagem Member is reported here. Specimens from the Ponta Grossa Formation, including the type material illustrated in Clarke (1913) were used for comparison.

In none of the studied exemplars the length (sag.)/width ratio values of cephalon determined by Simões et al. (2009) for B. notica (0.42-0.45) were observed - most ratios are between 0.6-0.8, a range that includes the array determined for B. herscheli (Simões et al., 2009). Specimens show a combination of characters typical to $B$. herscheli and $B$. notica, evidencing that these features should not be considered alone when identifying a Bumeisteria fossil. Curiously, the specimen AMNH 51007 (Fig. 2), from the Fox Bay Formation of Falkland Islands presented all the characters that fit in $B$. notica parameters. The exemplar is treated here as $B$. cf. notica, but 
further investigations to the Devonian of the Falkland Islands are necessary to confirm this suggestion.

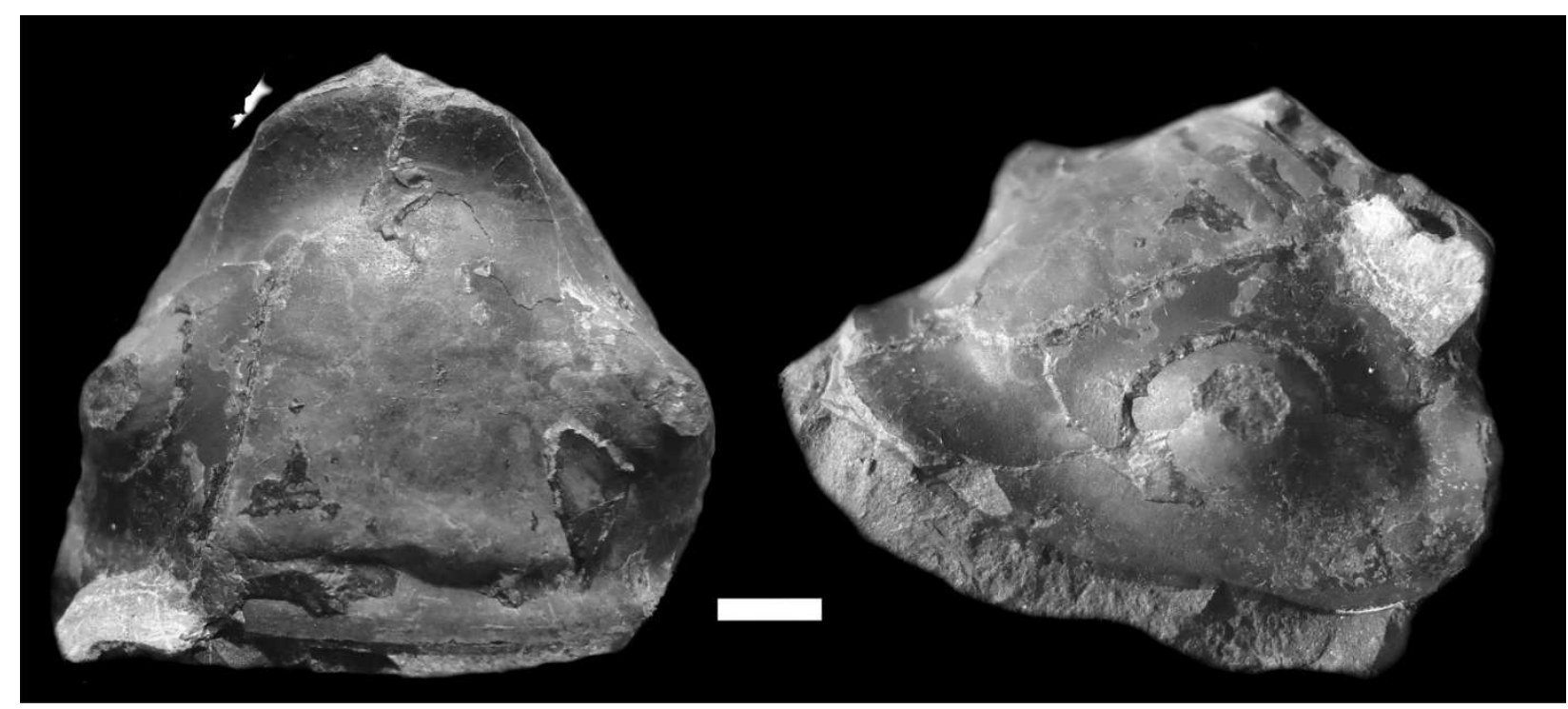

Figure 2: Burmeisteria cf. notica (AMNH 51007), from Fox Bay Formation, Falkland Islands. Scale bar represents $1 \mathrm{~cm}$.

\section{B. herscheli in the Parnaíba Basin?}

Some homalonotids from Picos and João Costa areas show urceolate glabella. The grading of this feature varies from discrete to strongly urceolate (Fig.3A-C). Medially constricted glabella in Parnaíba homalonotids is present in the description of $B$. notica from Picos and Pimenteiras areas by Carvalho (1995: 44). The specimens, however, lack tuberculation, though their exoskeletons present detailed surface preservation. This indicates that these fossils were not subjected to intense weathering processes which would efface the ornamentation. Due to the lack of tuberculation, the exemplars are regarded here as B. notica. This suggests that this species, as B. herscheli, varies in its glabella morphology. 


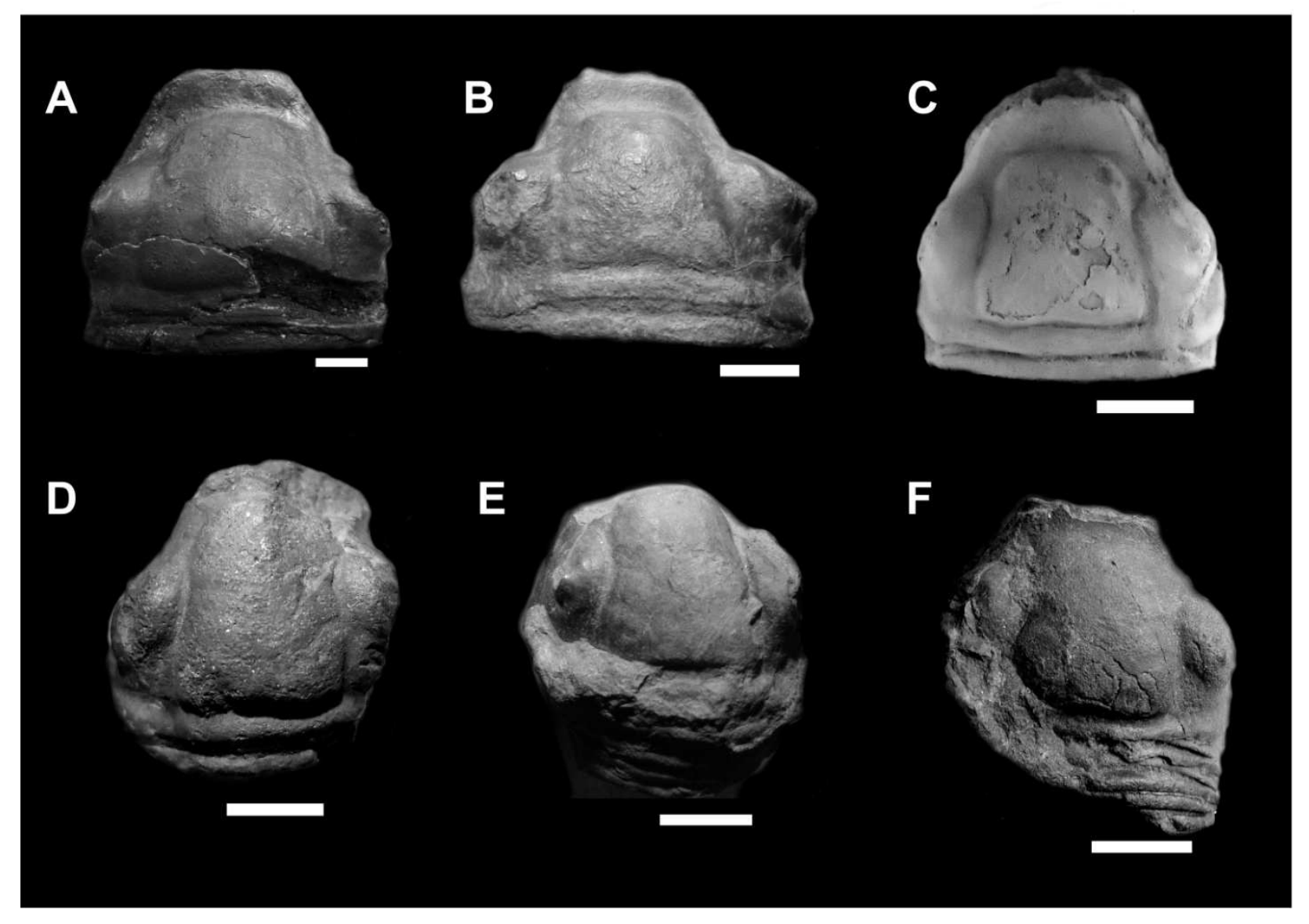

Figure 3: Burmeisteria notica from Pimenteiras Formation of the Piauí State, from the area of Picos city (A) and from São João Vermelho (B-F). Note the variation in glabella shape, ranging from trapezoidal (A-B) to urceolate (C-F). A) 5005-DGM; B) FUMDHAM specimen (no identification code);C) FUMDHAM 197602; D) FUMDHAM LPF-1; E) FUMDHAM specimen (no identification code); F) FUMDHAM LPF-5. Scale bars represent $1 \mathrm{~cm}$.

\section{Burmeisteria in the Passagem Member}

Ponciano et al. (2012) reported the presence of homalonotid trilobites in the Passagem Member from three localities within the Picos and Pimenteiras cities area: Oiti, Rio Banguê and Barreiro Branco. Here we report the occurrence of homalonotids in the Oeiras area, in the same region as that where other homalonotid occurrences were reported. The sample 136-Tr (IG-UFRJ) corresponds to a thoracopygidium of a probable Burmeisteria, considering the faint-impressed segments on pygidium (Fig. 4A-C). The Passagem Member is now considered a proximal sandier facies of the Pimenteira Formation (Ponciano et al., 2012). This find has important taphonomic implications, as the presence of a partially articulated fossil suggests little material 
reworking prior to its final burial. Homalonotid trilobites most likely lived in shallower waters also attested by Simões et al. (2009) for Burmeisteria in the Paraná Basin.

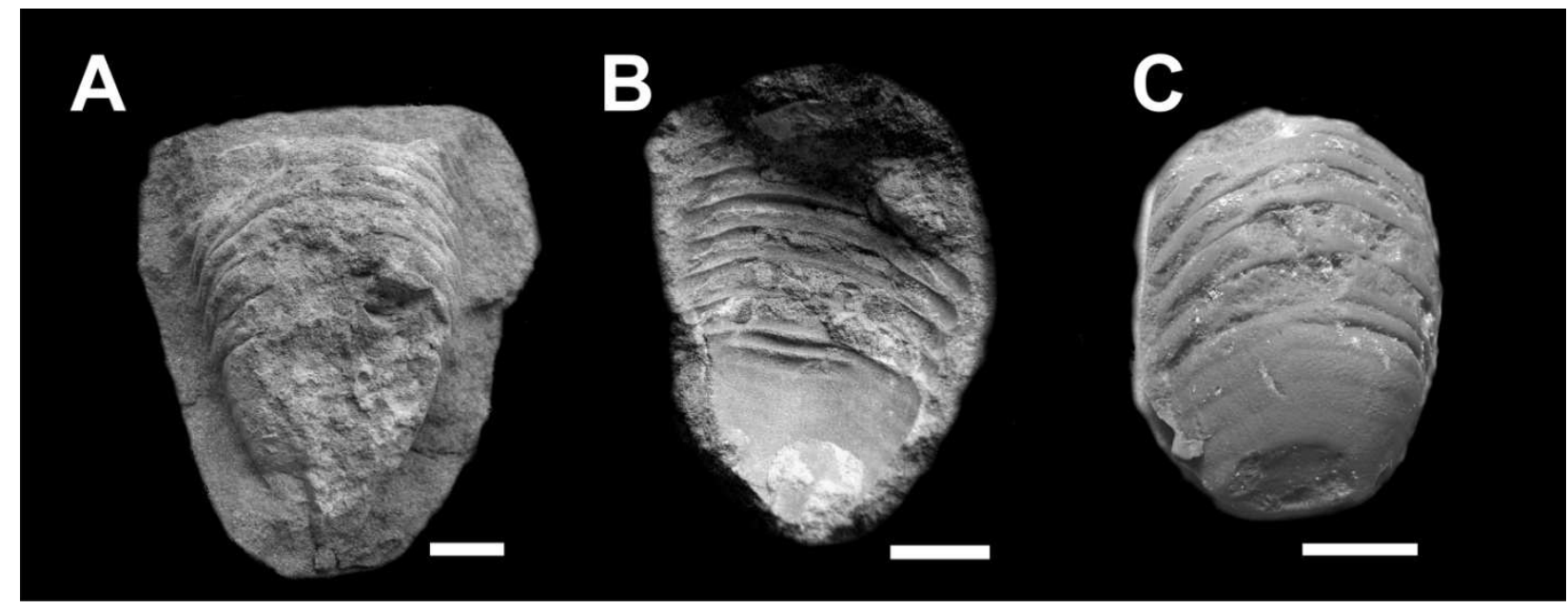

Figure 4: Thoracopygidium of Burmeisteria notica from the Passagem Member, Pimenteiras Formation in the Oeiras area, Piauí State (136-Tr). A) Cast; B) external mold; C) Clay cast from B. Scales represent $1 \mathrm{~cm}$.

\section{FINAL CONSIDERATIONS}

Based on the preliminary results exposed above, differences between B. notica and B.herscheli are slighter than previously thought. The most evident divergence seems to be the presence/absence of exoskeleton ornamentation which, in turn, is not always present, as taphonomic modifications can obliterate it (Simões et al., 2009). Simões et al. (2009) consider the urceolate glabella exclusive to $B$. herscheli. If this was taken into consideration, then specimens with urceolate glabella from the Pimenteira Formation should be better included in this species. However, the lack of tubercles in exemplars with no signs of weathering indicates they should be left in B. notica species, following previous classifications of homalonotids from this area (Castro, 1968; Carvalho, 1995). These questions reflect the still poor understanding of fossils from the Parnaíba Basin, and confirm the urgency in collecting more specimens in order to adequately observe the variation within $B$. notica. Likewise, the presence of $B$. cf. notica in the Fox Bay Formation, where the only known homalonotid is B. herscheli, calls to the necessity of new field works in the area, especially because the quantity of homalonotid fossils in the Falkland Islands is still low and biased towards larger individuals (Carvalho, 2006). 


\section{REFERENCES}

Caputo, M.V., Iannuzzi, R., and Fonseca, V.M.M. 2005. Bacias sedimentares brasileiras: Bacia do Parnaíba. Phoenix 81: 1- 6.

Carvalho, M.G.P. 1995. [Trilobitas devonianos da Bacia do Parnaíba (formações Pimenteira, Cabeças e Longá). PhD Thesis, Programa de Pós-graduação em Geologia, Universidade Federal do Rio de Janeiro, Rio de Janeiro, 132 p., Unpublished].

Carvalho, M.G.P. 2006. Devonian trilobites from the Falkland Islands. Palaeontology $4921-$ 34.

Carvalho, M.G.P., Edgecombe, G.D., and Lieberman, B.S. 1997. Devonian calmoniid trilobites from the Parnaíba Basin, Piauí State, Brazil. American Museum Novitates 3192: 1- 11.

Castro, J.S. 1968. Trilobitas da Formação Pimenteiras, Devoniano do estado do Piauí. Anais da Academia Brasileira de Ciências 40: 481- 489.

Clarke, J. M. 1913. Fósseis devonianos do Paraná. Monografia do Serviço Geológico e Mineralógico do Brasil 1: 1-353.

Cooper, M.R. 1982. A revision of the Devonian (Emsian - Eifelian) trilobita from the Bokkeveld Group of South Africa. Annals of the South African Museum 89: 1- 174.

Fonseca, V.M.M., and Melo, J.H.G. 1987. Ocorrência de Tropidoleptus carinatus (Conrad) (Brachiopoda, Orthida) na Formação Pimenteira, e sua Importância Paleobiogeográfica. $10^{\circ}$ Congresso Brasileiro de Paleontologia (Rio de Janeiro), Proceedings: 505- 537.

Grahn, Y., Melo, J.H.G., and Loboziak, S. 2006. Integrated Middle and Late Devonian miospore and chitinozoan zonation of the Parnaíba Basin, Brazil: an update. Revista Brasileira de Paleontologia 9: 283-294.

Grahn, Y., Young, C., and Borghi, L. 2008. Middle Devonian chitinozoan biostratigraphy and sedimentology in the eastern outcrop belt of the Parnaíba Basin, northeastern Brazil. Revista Brasileira de Paleontologia 11: 137-146.

Harrington, H.J., Henningsmoen, G., Howell, B.F., Jaanusson, V., Lochman-Balk, C., Moore, R.C., Poulsen, C., Rasetti, F., Richter, E., Schmidt, H., Sdzuy, K., Struve, W., Størmer, L., Stubblefield, C.J., Tripp, R., Weller, J.M., and Whittington, H.B.1959. Trilobita. In: R.C. 
Moore (Ed.), Treatise on Invertebrate Paleontology Part O. Arthropoda, Geological Society of America and University of Kansas Press, p. O38 - O526.

Kegel, W. 1953. Contribuição para o estudo do Devoniano da Bacia do Parnaíba. Boletim da Divisão de Geologia e Mineralogia 141: 1- 48.

Leme, J.M., Meira, F.van E., Stasi, A.M.D., and Soares, S.P. 2013. The occurrence of Phacopida trilobites from Pimenteira Formation at João Costa, Piauí, Brazil. Geologia USP - Série Científica 13: 17-22.

Lieberman, B. S. 1993. Systematics and biogeography of the "Metacryphaeus Group" Calmoniidae (Trilobita, Devonian), with comments on adaptative radiations and the geological history of the Malvinokaffric Realm. Journal of Paleontology 67: 549-570.

Lieberman, B. S., Edgecombe, G. D., and Eldredge, N. 1991, Systematics and biogeography of the "Malvinella Group", Calmoniidae (Trilobita,Devonian). Journal of Paleontology 65: $824-843$.

Meira, F. van E., Carbonaro, F.A., Ghilardi, R.P., and Leme, J. M. in press. The "Metacryhaeus tuberculatus Group" (Trilobita, Calmoniidae) from the Devonian of the Parnaíba Basin.

Melo, J.H.G., 1985. [A província Malvinocáfrica no Devoniano do Brasil: estado atual dos conhecimentos. Master's Dissertation, Programa de Pós-graduação em Geologia, Universidade Federal do Rio de Janeiro, Rio de Janeiro, 890 p., Unpublished].

Murchison, R.I. 1839. The Silurian System, founded on geological researches in the counties of Salop, Hereford, Radnor, Montgomery, Caermarthen, Brecon, Pembroke, Monmouth, Worcester, Gloucester and Stafford: with descriptions of the coalfields and overlying formations. London, John Murray 2: 568-768.

Plummer, F. 1948. Estados do Maranhão e Piauí. In: BRASIL, Conselho Nacional do Petróleo. Relatório de 1946: 87-134.

Ponciano, L.C.M.O., Fonseca, V.M.M., and Machado, D.M.C. 2012. Taphofacies analysis of late early Givetian fossil assemblages of the Parnaíba Basin (State of Piauí, northeast Brazil). Palaeogeography, Palaeoclimatology, Palaeoecology 326: 95-108.

Popp, M.T.B., Coimbra, J., and Hauch, A. 1996. Revisão do gênero Paracalmonia Struve, 1958 (pro Proboloides Clarke, 1913), Trilobita - um ensaio de sistemática filogenética. Gaia 12: 19-32. 
Salter, J.W. 1864. A monograph of the British trilobites from the Cambrian, Silurian and Devonian formations Part 1. Monograph of the Palaeontographical Society: 1-80.

Salter, J. W. 1865. A monograph of the British trilobites from the Cambrian, Silurian and Devonian formations Part 2. Monograph of the Palaeontographical Society: 1-80.

Saul, J. 1965. Trilobita. Antartic Research Series 6: 241-281.

Sdzuy, K. 1957. Bemerkungen zur Familie Homalonotidae (mit der Beschreibung einer neuen Art von Callymenella). Senckenbergiana lethaea 38: 275-290.

Simões, M.G., Leme, J.M., Soares, S.P. 2009. Systematics, taphonomy, and paleoecology of homalonotid trilobites (Phacopida) from the Ponta Grossa Formation (Devonian), Paraná Basin, Brazil. Revista Brasileira de Paleontologia 12 (1): 27-42.

Soares, S.P., Simões, M.G., and Leme, J.M. 2008. Metacryphaeus rotundatus, um novo elemento da fauna de trilobites Calmoniidae (Phacopida) da Formação Ponta Grossa, (Devoniano), Bacia do Paraná, Brasil. Geologia USP - Série Científica 8 (1): 15- 24.

Vaz, P.T., Rezende, N.G.A.M., Wanderley Filho, J.R., and Travassos, W.A.S. 2007. Bacia do Parnaíba. Boletim de Geociências da Petrobras 15: 253 
ANEXO VI

THE OCCURRENCE OF PHACOPIDA TRILOBITES FROM PIMENTEIRA FORMATION AT JOÃO COSTA, PIAUÍ, BRAZIL.

Artigo Publicado no Periódico Geologia USP - Série Científica 


\title{
The occurrence of Phacopida trilobites from Pimenteira Formation at João Costa, Piauí, Brazil A ocorrência de trilobitas Phacopida da Formação Pimenteira em João Costa, Piauí, Brasil
}

\author{
Juliana de Moraes Leme', Felipe van Enck Meira², Andre Mori Di Stasi², Sabrina Pereira Soares ${ }^{3}$ \\ ${ }^{1}$ Departamento de Geologia Sedimentar e Ambiental, Instituto de Geociências, Universidade de São Paulo - USP, Rua do Lago \\ 562, CEP 05508-080, São Paulo, SP, BR (leme@usp.br) \\ 2Programa de Pós-graduação em Geoquímica e Geotectônica, Instituto de Geociências, Universidade de São Paulo - USP, São \\ Paulo, SP, BR (fvemeira@gmail.com; andr_mori@hotmail.com) \\ ${ }^{3}$ Departamento de Educação, Faculdade de Ciências, Universidade Estadual Paulista "Júlio de Mesquita Filho" - UNESP, Bauru, \\ SP, BR (spereirasoares@yahoo.com.br)
}

Recebido em 19 de outubro de 2012; aceito em 10 de maio de 2013

\begin{abstract}
The marine macroinvertebrate fossils from the Devonian of the Parnaíba Basin are among the most diverse of Paleozoic of Brazil. In recent years, most revisions on these fossils involved trilobites from the Pimenteira and Cabeças formations, in the region of Picos and Pimenteiras cities, in the Piauí State. This survey reports the occurrence of Devonian trilobites from the Pimenteira Formation cropping out at João Costa city, in the region of Serra da Capivara National Park, southeastern Piauí State, where two species were recognized: Burmeisteria notica Clarke, 1913 and Metacryphaeus cf. australis Clarke, 1913. The wide occurrence of these genera in the Malvinokaffric Realm shows that transgressive events during part of the Devonian favored the faunal communication between the Andean, Brazilian and South African provinces through the reduction of geographical barriers. Finally, although the still preliminary character of the studies on Pimenteira Formation trilobites, this unit is already providing new paleontological data that contribute to the understanding of these fossil assemblages.
\end{abstract}

Keywords: Calmoniidae; Homalonotidae; Devonian; Parnaíba Basin; Serra da Capivara National Park.

\section{Resumo}

Os fósseis de macroinvertebrados marinhos do Devoniano da Bacia do Parnaíba estão entre os mais diversos do Paleozoico do Brasil. Nos últimos anos, a maioria das revisões sobre esses fósseis envolveu trilobitas das formações Pimenteira e Cabeças, na região das cidades de Picos e Pimenteiras, no Estado do Piauí. Esta pesquisa relata a ocorrência de trilobitas devonianos da Formação Pimenteira, aflorante na cidade de João Costa, na região do Parque Nacional da Serra da Capivara, no sudeste do Piauí, onde duas espécies foram reconhecidas: Burmeisteria notica Clarke, 1913 e Metacryphaeus cf. australis Clarke, 1913. A ampla ocorrência destes gêneros no Domínio Malvinocáfrico mostra que eventos transgressivos durante parte do Devoniano favoreceu a comunicação entre as faunas das províncias Andina, Brasileira e Sul-africana, através da redução das barreiras geográficas. Finalmente, apesar do caráter ainda preliminar dos estudos sobre trilobitas da Formação Pimenteira, esta unidade continua fornecendo novos dados paleontológicos que contribuem para o entendimento destas assembleias fósseis.

Palavras-chave: Calmoniidae; Homalonotidae; Devoniano; Bacia do Parnaíba; Parque Nacional da Serra da Capivara. 


\section{INTRODUCTION}

The marine macroinvertebrate fauna from the Devonian of the Parnaíba Basin is among the most diversified of Paleozoic of Brazil. It includes brachiopods, trilobites, ostracods, pelecypods, gastropods, hyolithids and tentaculitids preserved together with elasmobranchii fishes and plants in siliciclastics of Pimenteira and Cabeças formations. The fauna in intermediate sections of the Pimenteira Formation, cropping out at Piauí State, is dominated by the Chonetoidea brachiopod Montsenetes cf. boliviensis Rachebouef, 1992 and also includes pelecypods, gastropods, trilobites and conularids (Castro, 1968; Melo, 1988; Carvalho, 1995; Siviero, 2002). Important revisions on this fauna have taken place in the last years, involving brachiopods (Fonseca, 2001, 2004; Gama Jr., 2008) and conularids (Siviero, 2002). Studies on trilobites from the Pimenteira Formation back Caster (1948), who cited the occurrence of homalonotids and dalmanitids. The area between the cities of Picos and Pimenteiras, where this unit outcrops, was later visited by Kegel (1953), who collected fossils and described the occurrence of the genera Homalonotus König, 1825 and Asteropyge Hawle and Corda, 1847. These taxa were later reclassified by Castro (1968); the former as Burmeisteria notica Clarke, 1913 and the latter, as Metacryphaeus australis Clarke, 1913. In general, Devonian trilobites of Parnaíba Basin were mainly treated as side subjects in stratigraphic (Kegel, 1953) and paleobiogeographic (Melo, 1985, 1988) studies. Nevertheless, more attention has been given to these fossils in the last twenty years, through detailed surveys on systematics and paleobiogeography (e.g. Carvalho, 1995; Carvalho et al., 1997).

The current survey reports the occurrence of trilobites from the Pimenteiras Formation at João Costa city (Figure 1), in the vicinities of Serra da Capivara National Park, southeastern Piauí State.

\section{GEOLOGIC SETTING}

The Pimenteira Formation (Neoeifelian - Frasnian; Santos and Carvalho, 2004; Grahn et al., 2006) is located near the base of the Canindé Group, underlain by the Itaim Formation and overlain by the Cabeças Formation (Figure 2). Although in many works the unit is referred as "Pimenteiras Formation" as the formation's name derives from the town of Pimenteiras, in the Piaui State

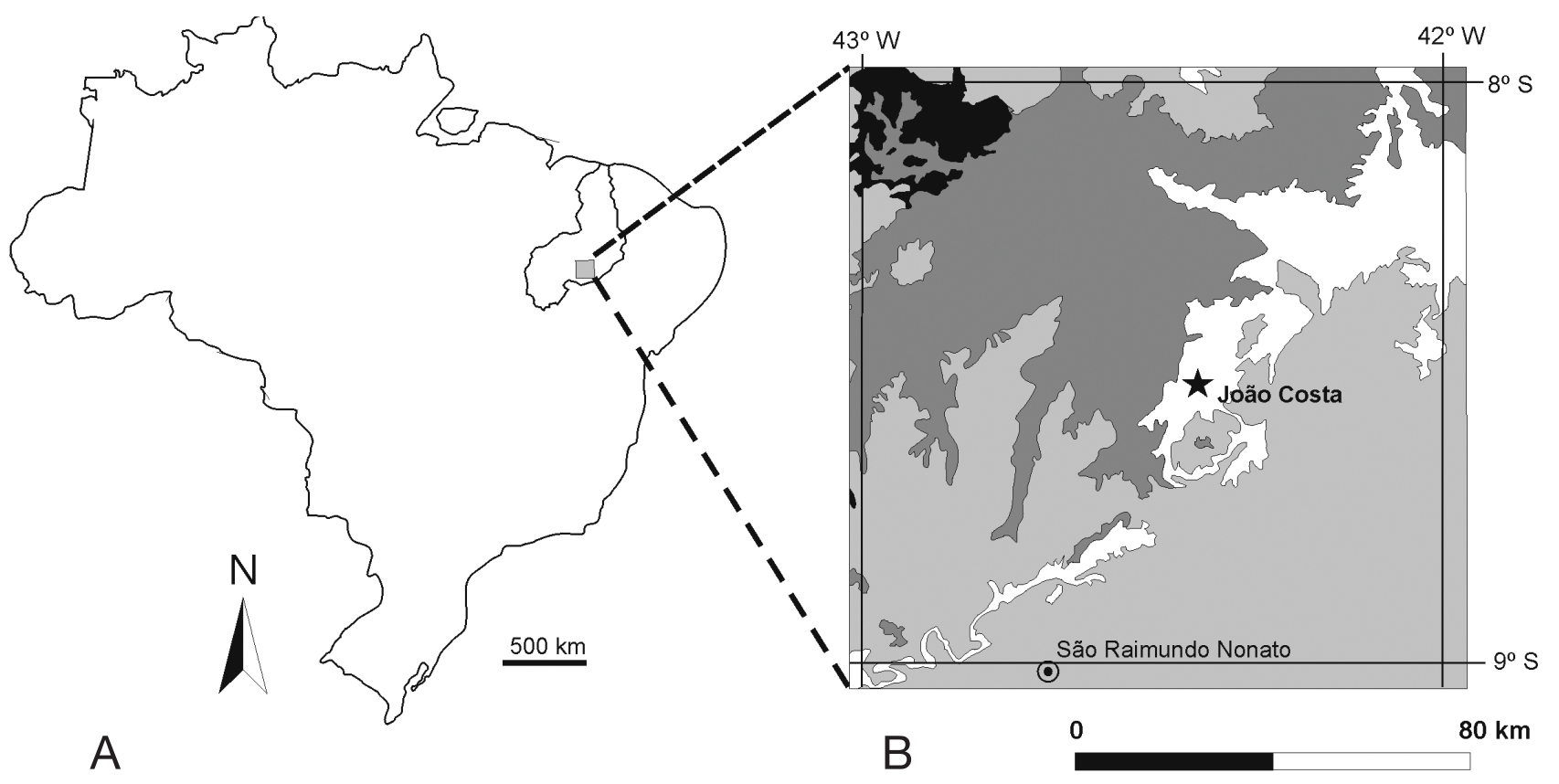

- Pimenteira Formation

- Cabeças Formation

- Longá Formation

Figure 1. Geological map showing the city of João Costa in the Piauí State, Brazil. Note that the town is positioned within the Pimenteira Formation. (B) modified from CPRM (2006). 
(Small, 1914; Plummer et al., 1948) we rather use the term "Pimenteira Formation" following Carvalho $(1995,2006)$, Carvalho et al. (1997); Silva and Fonseca (2005). The unit marked the greatest marine transgression in the Parnaíba Basin, and consists on siderite and pyrite-rich dark (reddish when weathered) shales interbedded with siltstones and fine sandstones deposited in a storm-dominated shallow marine platform (Della Fávera, 1990; Góes and Feijó, 1994). The shales are often bioturbated, and contain iron oolites and phosphatic and hematitic concretions (Della Fávera, 1990). The fossils are often found as molds and casts in iron oxide concretions (Melo, 1988).

\section{MATERIALS AND METHODS}

Forty exemplars from outcrops of the Pimenteira Formation (Figure 2) at the localities of Morro do Ranulfo and Morro do Joaquim (23L UTM 9053313, 781243), in João Costa city, about $70 \mathrm{~km}$ from São Raimundo Nonato town, southeastern Piauí State were examined (Table 1). The fossils, preserved in ferruginous concretions, are deposited at the Fundação Museu do Homem Americano (FUNDHAM) scientific collection, in São Raimundo Nonato city. The preparation of the specimens followed the techniques used in the study of marine macrofossils mentioned in Feldmann et al. (1989) and Nobre and Carvalho (2010). The systematic identification and the morphologic terminologies for trilobites followed Eldredge and Branisa (1980), Lieberman et al. (1991), Lieberman (1993), Whittington et al. (1997) and Sandford (2005).

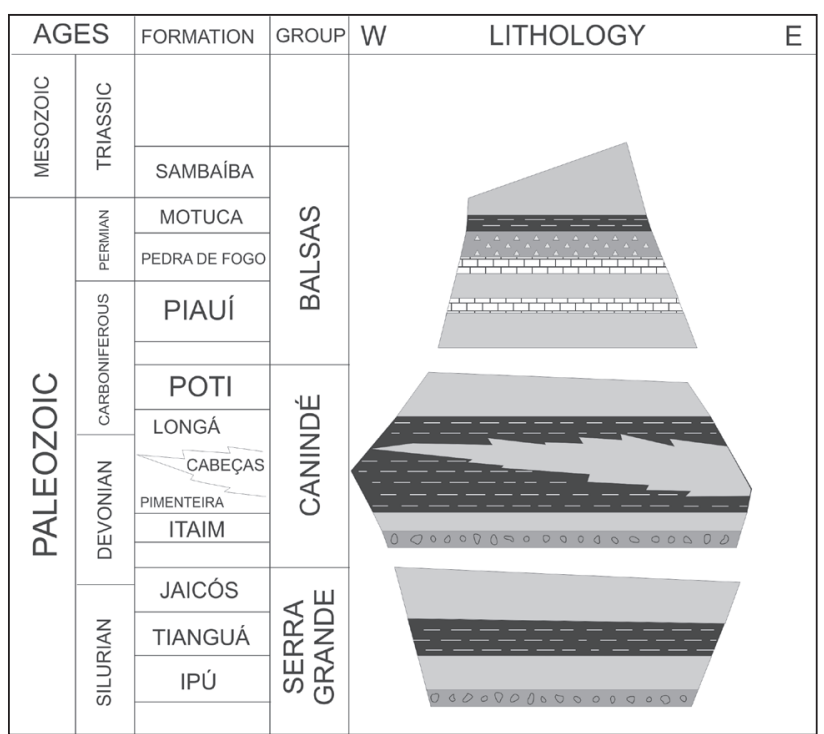

Figure 2. Stratigraphic section of the Paleozoic and early Mesozoic of the Parnaíba Basin. Modified from Góes (1995).
The fossils were analyzed with a Zeiss stereomicroscope and photographed with a Canon SX10 digital camera. These steps were carried out at the Laboratório de Estudos Paleobiológicos of the Instituto de Geociências da Universidade de São Paulo (IGc/USP).

\section{RESULTS AND DISCUSSION}

From the analyzed fossils, it was possible to discern two trilobite species: Burmeisteria notica and Metacryphaeus cf. australis (Figures $3 \mathrm{~A}-3 \mathrm{G}$ ). The material also includes one specimen of Rusophycus Hall, 1852 (Figure 3H), already recognized in the area (Silva et al. 2011). Both trilobite species were described from the Pimenteira Formation in the region between Picos and Pimenteiras cities (Castro, 1968; Carvalho, 1995) and are also found in the Ponta Grossa Formation, Paraná Basin (Clarke, 1913; Carvalho et al., 1987). The occurrence of Burmeisteria Salter, 1865 and Metacryphaeus Reed, 1907 in João Costa town spreads the already wide distribution of these genera within the Malvinokaffric Realm, which also includes Bolivia (Icla, Bélen, Gamoneda, Sicasica and Catavi formations), Argentina (Lipéon Formation), South Africa (Gydo and Voorstehoek formations), Falkland Islands (Fox Bay Formation) and Uruguay (Cordobés Formation) (Kozlowski, 1923; Reed, 1925; Branisa, 1965; Copper, 1977; Eldredge and Ormiston, 1979; Cooper, 1982; Lieberman, 1993; Carvalho, 1995, 2006; Carvalho et al., 1987; Carvalho et al., 1994).

Although the distribution of homalonotid trilobites extends to the Old World Realm, Burmeisteria consists on the most common genus in the Malvinokaffric Realm (Simões, et al., 2009). Contrarily, Metacryphaeus is considered an endemic genus for this paleobiogeographic province (Eldredge and Ormiston, 1979). The wide occurrence of Burmeisteria and Metacryphaeus in transgressive deposits within the Malvinokaffric context indicates that faunal interchanging between Andean, Brazilian and South African provinces took place during part of the Devonian, favored by the reduction of geographic barriers.

Table 1. Mode of preservation and number of samples of B. notica and $M$. cf. autralis.

\begin{tabular}{lcc}
\hline SPECIES/PRESERVATION & B. notica & M. cf. autralis \\
\hline Complete & 9 & - \\
Thorax-pygidium & 3 & 3 \\
Cephalon-thorax & 7 & - \\
Cephalon & 2 & - \\
Pygidium & 9 & 1 \\
Thorax & 6 & - \\
TOTAL & 36 & 4 \\
\hline
\end{tabular}




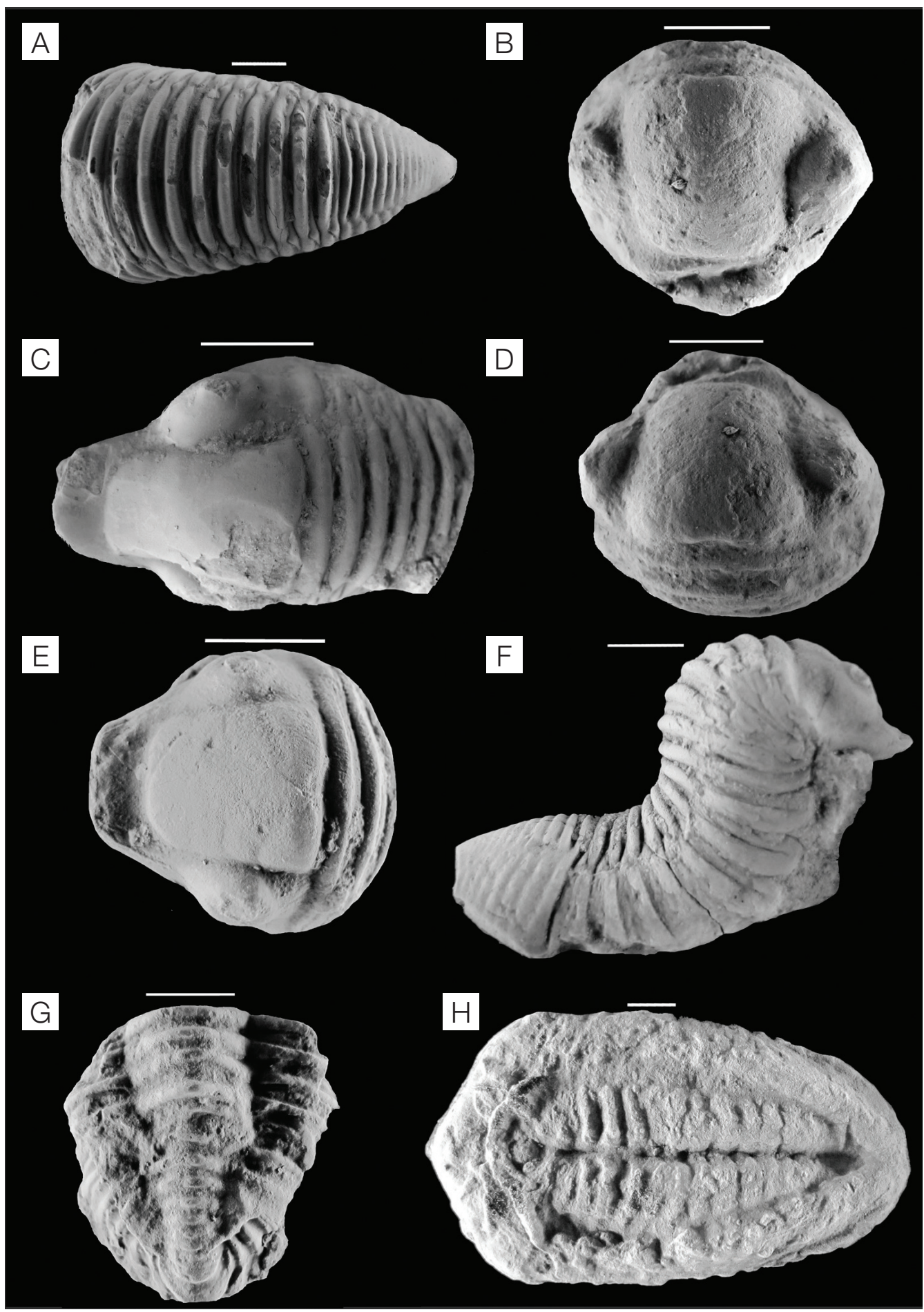

Figure 3. Specimens of Burmeisteria notica (A - F), Metacryphaeus cf. australis (G) and Rusophycus (H) from the Pimenteira Formation at João Costa city, southeastern Piauí State, and deposited at the Fundação Museu do Homem Americano scientific collection, under the numbers 169056 (A), 169045 (B, D and E), 169039 (C), 169044-1 (F), 169046 (G), and $872-$ $113981(\mathrm{H})$. Scale bars represent $1 \mathrm{~cm}$. 


\section{FINAL CONSIDERATIONS}

Though relatively recent, studies on trilobites from the Pimenteira Formation are already providing new paleontological, which allow a more precise comparison between fossil assemblages in this basin and those from other localities in the Malvinokaffric Realm.

\section{ACKNOWLEDGEMENTS}

The authors are grateful to Niède Guidon (Fundação Museu do Homem Americano), who kindly lent the fossils presented in this survey, Dr. Luiz Eduardo Anelli for the assistance in photos, and two anonymous referees for the comments.

\section{REFERENCES}

Branisa, L. (1965). Los fosiles guias de Bolivia. I. Paleozoico. Servicio Geologico de Bolivia, Boletin, 6, 1-282.

Carvalho, M. G. P. (1995). Trilobitas devonianos da Bacia do Parnaiba (Formações Pimenteira, Cabeças e Longá). Tese (Doutorado). Rio de Janeiro: Instituto de Geociências - UFRJ.

Carvalho, M. G. P. (2006). Devonian trilobites from the Falkland Islands. Palaeontology, 49(1), 21-34.

Carvalho, M. G. P., Edgecombe, G. D., Lieberman, B. S. (1997). Devonian Calmoniid trilobites from the Parnaíba Basin, Piauí State, Brazil. American Museum Novitates, 3192, 1-11.

Carvalho, M. G. P., Melo, J. H. G., Quadros, L. P. (1987). Trilobitas devonianos do flanco noroeste da Bacia do Paraná. X Congresso Brasileiro de Paleontologia, v. 2, 545-565. Rio de Janeiro: SBP.

Caster, K. E. (1948). Excursão Geológica ao Estado do Piauí. Mineração e Metalurgia, 12(72), 271-272.

Castro, J. S. (1968). Trilobitas da Formação Pimenteiras, Devoniano do Estado do Piauí. Annais da Academia Brasileira de Ciências, 40(4), 481-489.

Clarke, J. M. (1913). Fósseis devonianos do Paraná. Monographia do Serviço Geológico e Mineralógico do Brasil, 1, 1-353.

CPRM. (2006). Serviço Geológico do Brasil. Mapa geológico do Estado do Piaui: Sistema de Informações
Geográficas - SIG. $2^{\mathrm{a}}$ versão. Teresina: 1 CD-ROM, Escala 1:1.000.000.

Cooper, M. (1982). A revision of the Devonian (EmsianEifelian) trilobita from Bokkeveld Group of South Africa. Annals of the South African Museum, 89, 1-174.

Copper, P. (1977). Paleolatitudes in the Devonian of Brazil and the Frasnian - Famennian mass extinction. Palaeogeography, Palaeoclimatology, Palaeoecology, 21, 165-207.

Della Fávera, J. C. (1990). Tempestitos da Bacia do Parnaiba: um ensaio holístico. Tese (Doutorado). Porto Alegre: Instituto de Geociências - UFRGS.

Eldredge, N., Branisa, L. (1980). Calmoniid trilobites of the Lower Scaphiocoelia Zone of Bolivia, with remarks on related species. Bulletin of the American Museum of Natural History, 165(2), 185-289.

Eldredge, N., Ormiston, A. R. (1979). Biogeography of Silurian and Devonian Trilobites of the Malvinokaffric Realm. In: A. J. Boucot, J. Gray (Eds.), Historical biogeography, plate tectonics, and changing environment. (147-167). Oregon State Univ. Press.

Feldmann, R. M., Chapman, R. E., Hannibal, J. T. (1989). Paleotechiniques. Knoxville: The Paleontological Society.

Fonseca, V. M. M. (2001). Brachiopoda (Stropheodontoidea, Chonetoidea e Delthyiridoidea) do Devoniano Médio das bacias do Amazonas e Parnaiba. Tese (Doutorado). Rio de Janeiro: Instituto de Geociências - UFRJ.

Fonseca, V. M. M. (2004). Chonetoidea (Brachiopoda, Strophomenata, Productida, Chonetidina) do Devoniano Médio das bacias do Amazonas e Parnaíba, Brasil. Arquivos do Museu Nacional, 62(2), 193-215.

Gama Jr., J. M. (2008). Braquiópodes da Formação Pimenteiras (Devoniano Médio/Superior), na região Sudoeste da Bacia do Parnaiba, Estado do Tocantins, Brasil. Dissertação (Mestrado). Brasília: Instituto de Geociências - UnB.

Góes, A. M. O. (1995). A Formação Poti (Carbonifero Inferior) da Bacia do Parnaíba. 1995. Tese (Doutorado). São Paulo: Instituto de Geociências - USP.

Góes, A. M. O., Feijó, F. J. (1994). Bacia do Parnaíba. Boletim de Geociências da Petrobrás, 8, 57-67. 
Grahn, Y., Melo, J. H. G., Loboziak, S. (2006). Integrated Middle and Late Devonian miospore and chitinozoan zonation of the Parnaíba Basin, Brazil: an update. Revista Brasileira de Paleontologia, 9(3), 283-294.

Kegel, W. (1953). Contribuição para o estudo do Devoniano da Bacia do Parnaíba. Boletim da Divisão de Geologia e Mineralogia, 141, 1-48.

Kozlowski, R. (1923). Faune Dévonienne de Bolivie. Annales de Paleontologie, 12(1/2), 1-112.

Lieberman, B. S. (1993). Systematics and biogeography of the "Metacryphaeus Group", Calmoniidae (Trilobita, Devonian), with comments on adaptative radiations and the geological history of the Malvinokaffric Realm. Journal of Paleontology, 67(4), 549-570.

Lieberman, B. S., Edgcombe, G. D., Eldredge, N. (1991). Systematics and biogeography of the "Malvinella Group", Calmoniidae (Trilobita, Devonian). Journal of Paleontology, 65(5), 824-843.

Melo, J. H. G. (1985). A Província Malvinocáfrica no Devoniano do Brasil: estado atual dos conhecimentos. 1985. Dissertação (Mestrado). Rio de Janeiro: Instituto de Geociências - UFRJ.

Melo, J. H. G. (1988). The Malvinokaffric realm in the Devonian of Brazil. In: McMillan, N. J., Embry, A. F., Glass, D. J. (Eds.), Devonian of the World, (v.1, 669-703). Calgary: Canadian Society of Petroleum Geologists.

Nobre, P. H., Carvalho, I. S. (2010). Fósseis: coleta e métodos de estudo. In: I.S. Carvalho (Ed.), Paleontologia, $3^{a}$ edição, (397-411). Rio de Janeiro: Interciência.

Plummer, F. B., Price, L. I., Gomes, F. A. (1948). Estados do Maranhão e Piauí. Conselho Nacional de Petróleo, Relatório 1946, 87-134.

Rachebouef, P. R. (1992). Los chonetáceos (brachiópodos) del Devónico boliviano: bioestratigrafia y datos taxonómicos complementarios. Revista Española de Paleontología, 7, 31-52.
Reed, F. R. C. (1925). Revision of the Fauna of the Bokkeveld Beds. Annals of the South African Museum, 22, 27-225.

Sandford, A. C. (2005). Homalonotid trilobites from the Silurian and Lower Devonian of south-eastern Australia and New Zealand (Arthropoda: Trilobita: Homalonotidae). Memoirs of Museum Victoria, 62(1), 1-66.

Santos, M.E. C. M., Carvalho, M. S. S. (2004). Paleontologia das bacias Parnaíba, Grajaú e São Luís. Programa Levantamentos Geológicos Básicos do Brasil,CPRM. Serviço Geológico do Brasil DIEDIG/DEPAT, Rio de Janeiro. Livro eletrônico em formato pdf, CD-Rom.

Silva, C. F., Fonseca, V. M. M. (2005). Hábitos de vida dos trilobitas das formações Maecuru e Ererê, Devoniano da Bacia do Amazonas, Brasil. Revista Brasileira de Paleontologia, 8(1), 73-82.

Silva, R. C., Dominato, V. H., Fernandes, A. C. S. (2011). Novas considerações sobre icnofósseis devonianos da Formação Pimenteira, Bacia do Parnaíba, Brasil. XXII Congresso Brasileiro de Paleontologia, 483-485. Natal: SBP.

Simões, M. G., Leme, J. M., Soares, S. P. (2009). Systematics, taphonomy and paleoecology of Homalonotid trilobites (Phacopida) from the Ponta Grossa Formation (Devonian), Paraná Basin, Brazil. Revista Brasileira de Paleontologia, 12(1), 27-42.

Siviero, F. N. (2002). Revisão sistemática das conulárias brasileiras. 2002. Dissertação (Mestrado). Rio de Janeiro: Instituto de Geociências - UFRJ.

Small, H. L. (1914). Geologia e supprimento de água subterranea no Piauhy e parte do Ceará. Rio de Janeiro: Inspectoria de Obras Contra as Seccas.

Whittingon, H. B., Chatterton, B. D. E., Speyer, S. E., Fortey, R. A., Owens, R. M., Chang, W. T., Dean, W. T., Jell, P. A., Lawrie, J., Palmer, A. R., Repina, L. N., Rushton, A. W. A., Shergold, J. H., Clarkson, E. N. K., Wilmot, N. V., Kelly, S. R. A. (1997). Treatise on Invertebrate Paleontology, Part O, Arthropoda 1, Trilobita. Revised edition. Lawrence: Geological Society of America and the University of Kansas Press. 
